\title{
Regional Mechanics of Demand Ischemia During Cardiac Stress Testing
}

\author{
A Dissertation \\ Presented to \\ the faculty of the School of Engineering and Applied Science \\ University of Virginia

\begin{abstract}
in partial fulfillment
of the requirements for the degree

Doctor of Philosophy

in Biomedical Engineering
\end{abstract} \\ by \\ Katherine McKenzie Parker \\ June 2013
}


(C) 2013 Katherine McKenzie Parker 


\section{Abstract}

Currently, the most effective non-invasive method for early diagnosis of coronary artery disease is the cardiac stress test. In this test, stress is induced by treadmill exercise or with a pharmacologic agent such as dobutamine, and myocardium with insufficient coronary flow reserve, usually caused by coronary stenosis, experiences an imbalance between oxygen supply and demand known as demand ischemia. This causes altered mechanical and electrical behavior that is often identified using cardiac imaging. The mechanical movement of the left ventricular (LV) wall, characterized clinically as "wall motion", is currently assessed qualitatively to identify abnormally-moving ischemic regions which may benefit from angioplasty. Advances in ultrasound and MRI may improve diagnosis by allowing more quantitative measures such as strain, wall thickening, or endocardial fractional shortening. However, little is understood about how these measures are affected by the severity of demand ischemia, coupling to adjacent myocardium, or the presence of previously-unrecognized myocardial infarction. A greater understanding of the factors that influence regional mechanics during stress testing would help determine the best way to detect the altered mechanics that indicate demand ischemia and coronary stenosis. The goal of this dissertation is to understand how regional demand ischemia affects regional mechanics and apply that knowledge to improve clinical stress testing.

A finite element model of the heart was used to examine how reduced force generation in the ischemic region, ischemic region size, and coupling to myocardium with increased contractility separately and jointly impact various measures of regional mechanics. Area strain and a measure of wall motion developed in our lab, three- 
dimensional fractional shortening (3DFS), were most sensitive to reduced force generation, while radial strain was affected most by the contractility of remote myocardium. The model also predicted that a novel measure of wall motion bulging, dyskinesia severity index (DSI), can separate infarcts from demand ischemia during stress testing. The ability of strain and 3DFS to detect a critical stenosis was evaluated in experimental canine dobutamine stress tests. The three-dimensional (3D) measures of area strain and 3DFS detected critical stenoses better than two-dimensional (2D) strain. Finally, 3DFS measured during clinical stress testing in patients at low risk for coronary artery disease displayed low variability, suggesting 3DFS may be effective for detecting demand ischemia in patients.

Another application in which the cardiac mapping techniques described here could improve treatment is in evaluating cardiac resynchronization therapy (CRT) nonresponders, which compromise $30-40 \%$ of CRT patients. The relationship between LV lead location and scar or regional mechanical function measured from pre-CRT cardiac imaging likely has an important impact on CRT response. A method to reconstruct the $3 \mathrm{D}$ coordinates of the lead from $2 \mathrm{D}$ fluoroscopic images and then map the LV lead position onto a pre-CRT imaging study was developed. The accuracy of the average mapped lead position was within $1.5 \mathrm{~cm}$. This method will allow clinicians to evaluate nonresponders and test hypotheses to quantitatively optimize LV lead location without specialized cardiac mapping equipment.

The work of this dissertation improves our understanding of the mechanical consequences of demand ischemia and interpretation of the functional stress test, and enables more quantitative image analysis during both stress testing and CRT evaluation. A more quantitative and empirical method for stress test interpretation and CRT evaluation will not only improve diagnostic utility, but enable better training, center-to- 
center repeatability, and clinical research methods for publishing regional function data. The significance of this work is that it will increase understanding of regional mechanical behavior during cardiac stress testing, and improve the diagnostic capability of the cardiac stress test by enabling quantitative diagnosis of mechanical dysfunction. 


\section{Table of Contents}

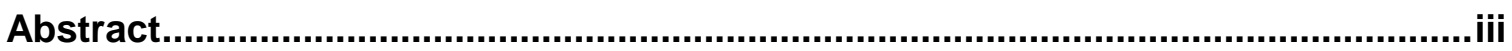

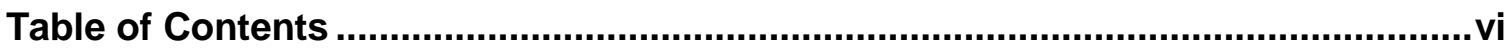

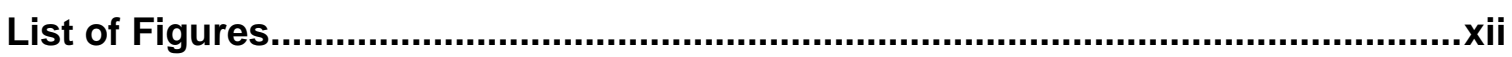

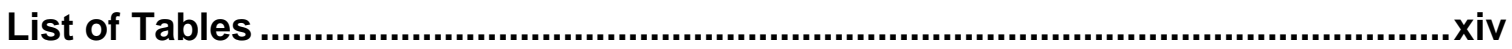

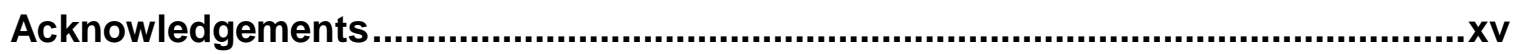

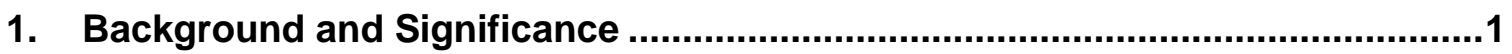

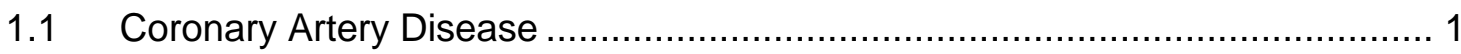

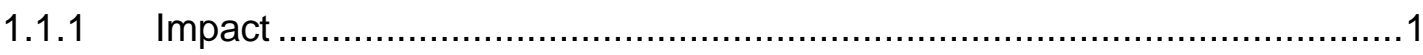

$1.1 .2 \quad$ Treatment

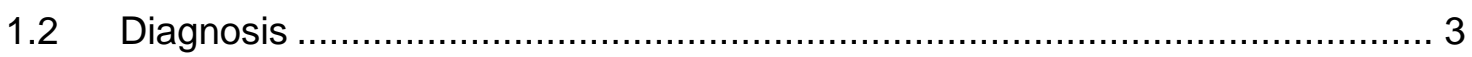

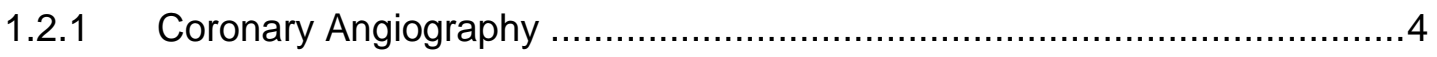

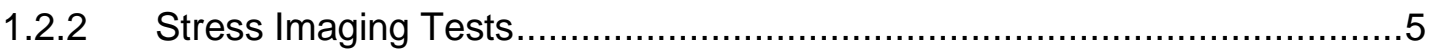

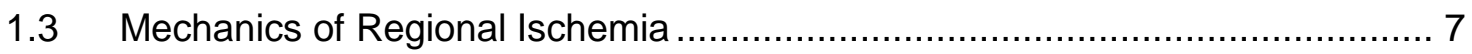

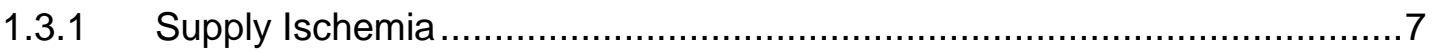

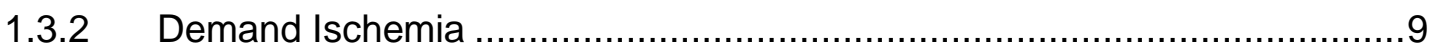

1.4 Measurement of Mechanics With Imaging ...............................................10

1.4.1 Cardiac Magnetic Resonance ……………...................................10

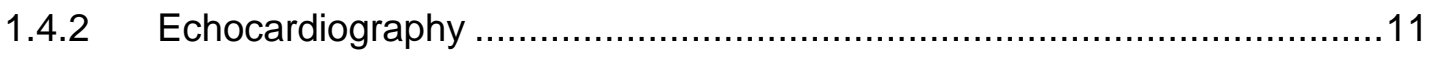

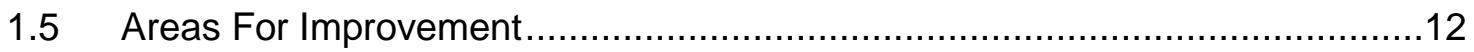

2. Measuring Cardiac Wall Motion From Noninvasive Imaging............................14 
2.1 Canine Model of Dobutamine Stress ................................................ 14

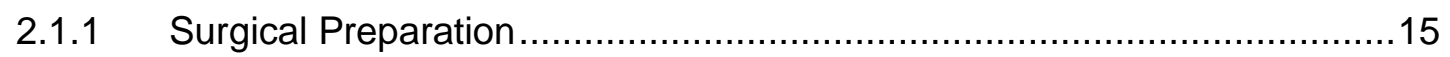

2.1.2 Assessment of Functional Data and Regional Strain ..........................16

2.1.3 Assessment of Regional Myocardial Blood Flow ...................................18

2.2 Quantitative Wall Motion Analysis ...................................................19

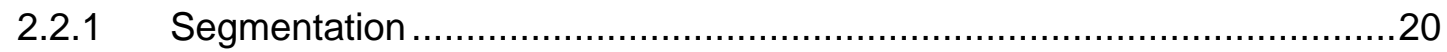

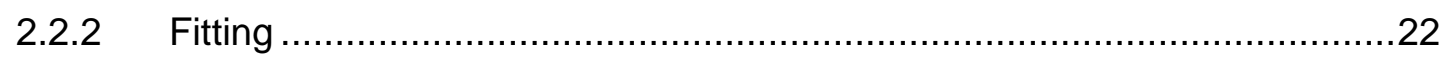

2.3 Interobserver Variability of 3DE Manual Segmentation .............................26

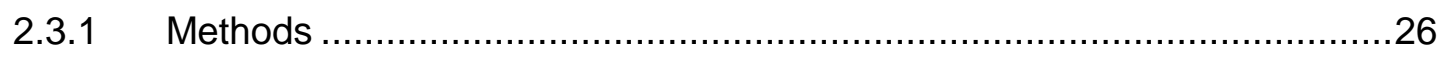

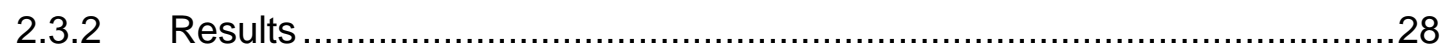

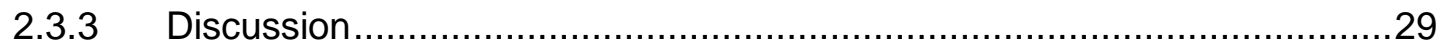

\section{Sensitivity of Mechanical Measures to Force Generation in the Ischemic}

Region and Surrounding Myocardium ..................................................................... 39

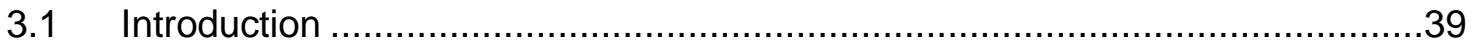

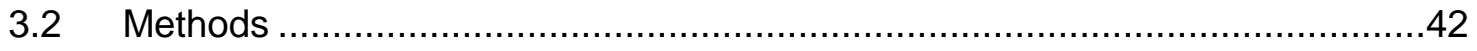

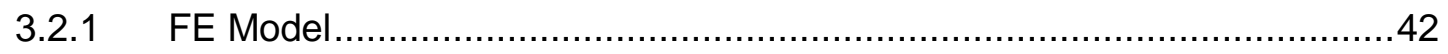

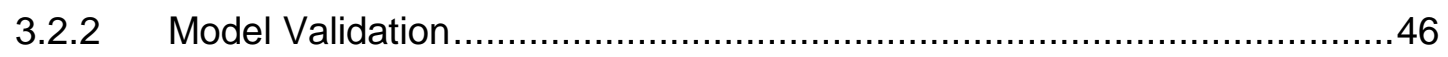

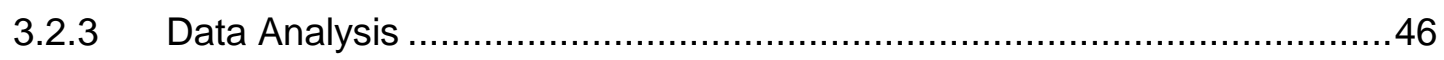

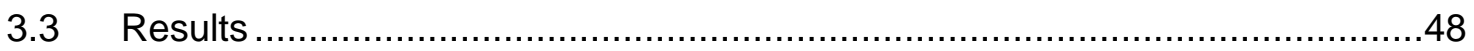

3.3.1 Model Validation................................................................... 48

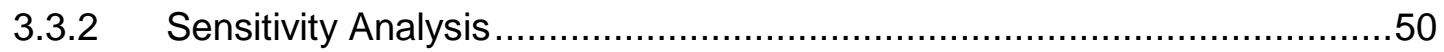


3.3.3 Size of Mechanical Abnormalities .................................................. 51

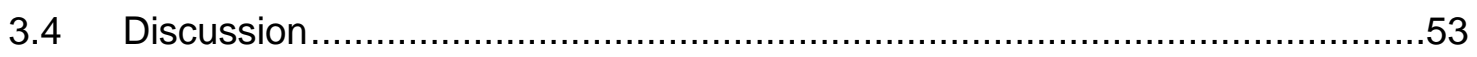

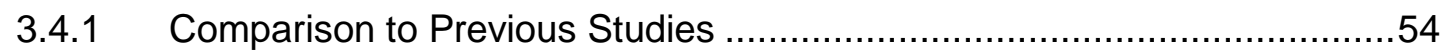

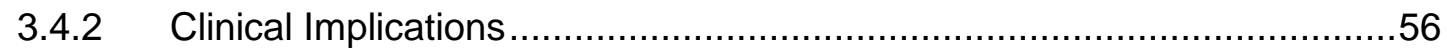

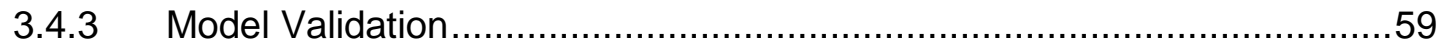

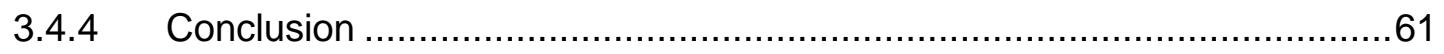

\section{Comparison of Quantitative Wall Motion Analysis and Strain for Detection of a} Critical Stenosis with Three-Dimensional Dobutamine Stress Echocardiography 76

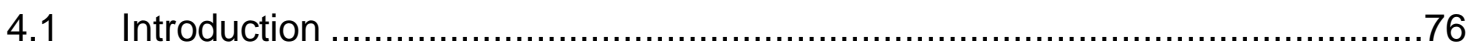

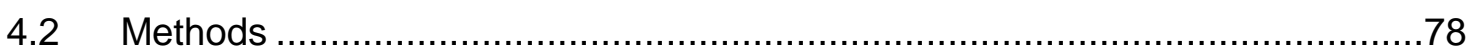

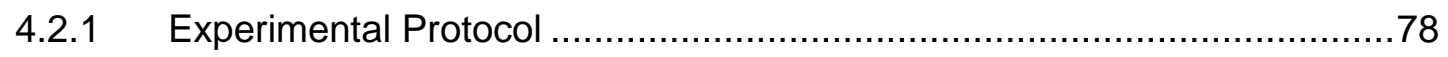

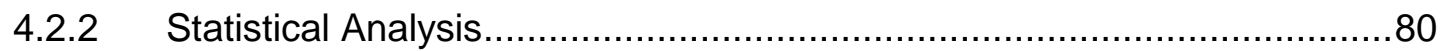

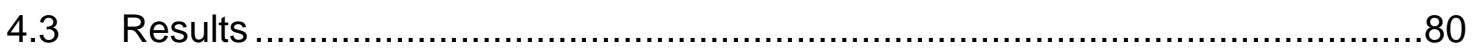

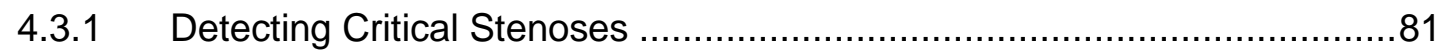

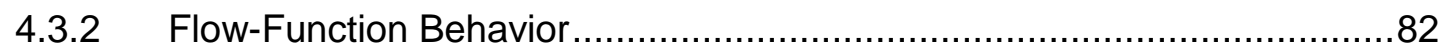

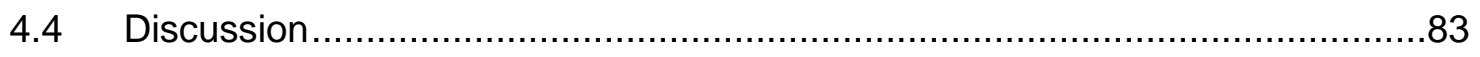

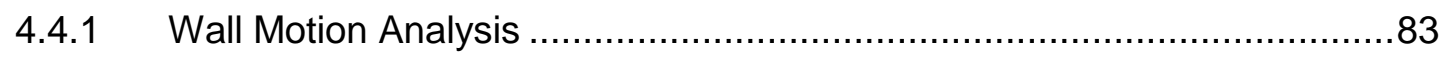

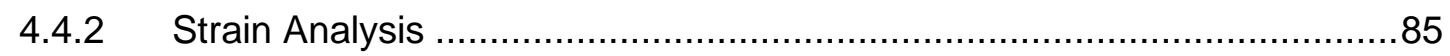

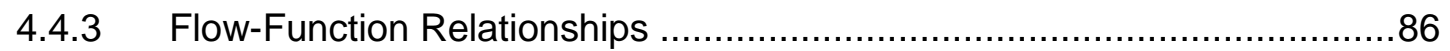

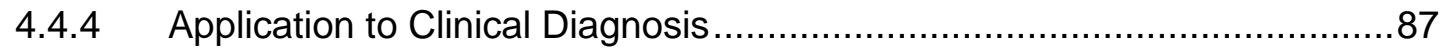

4.4.5 Comparison to Model Results..................................................... 88 
4.4.6 Sources of Error and Limitations ................................................ 90

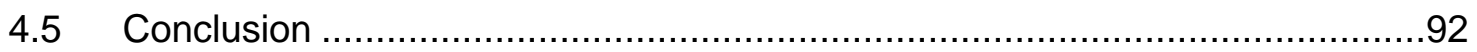

5. Evaluation of Quantitative Wall Motion Analysis for Distinguishing Between

Stiff Infarcts and Acute Ischemia .....................................................................103

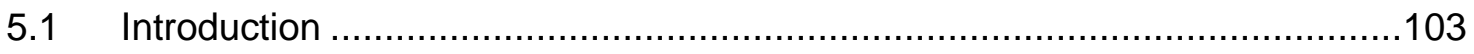

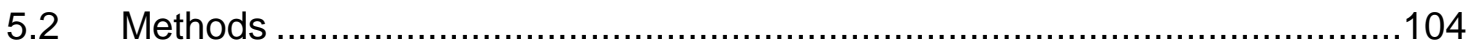

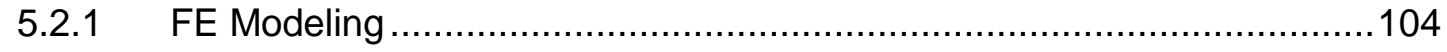

5.2.2 In Vivo Wall Motion Analysis ................................................... 106

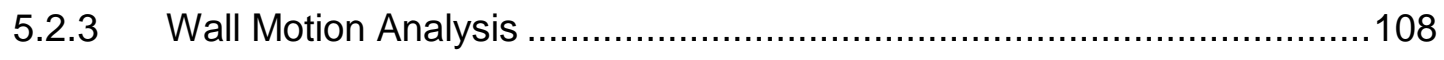

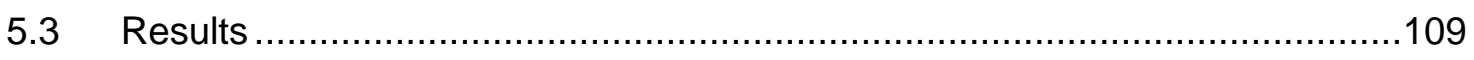

5.3.1 Acute Ischemia vs. Infarcts At Rest .......................................... 109

5.3.2 Acute Ischemia vs. Infarcts During Stress .....................................110

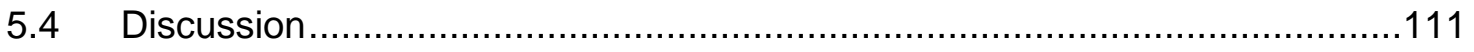

5.4.1 Acute Ischemia vs. Infarcts At Rest ......................................... 111

5.4.2 Acute Ischemia vs. Infarcts During Stress .................................... 114

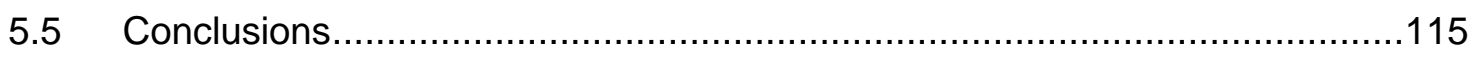

6. Wall Motion in Low-Risk Patients Undergoing Dobutamine Stress

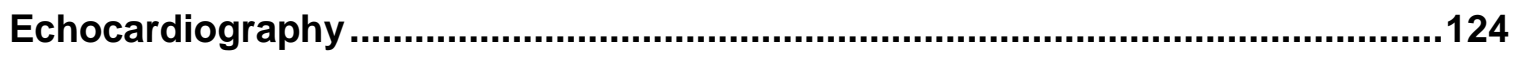

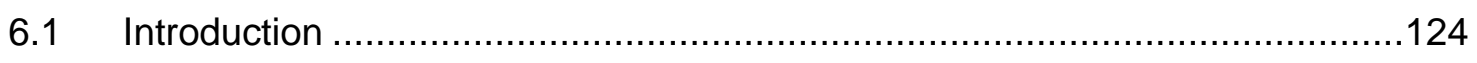

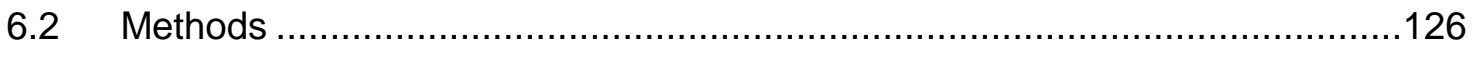

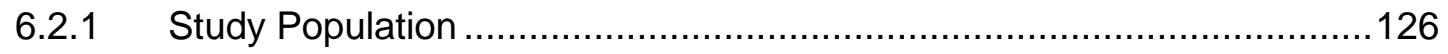




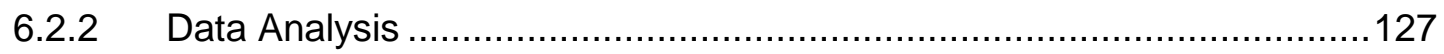

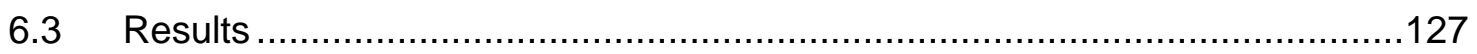

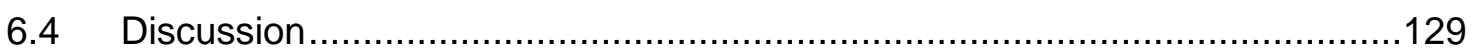

6.4.1 Perception vs. Reality in DSE...................................................... 130

6.4.2 Application of 3DFS to Clinical Cases ............................................ 132

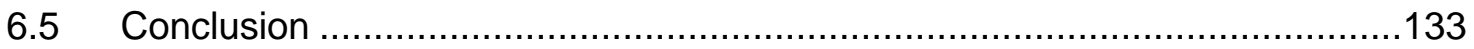

\section{Applications of Cardiac Mapping: Relating Lead and Infarct Location to} Evaluate Cardiac Resynchronization Therapy Nonresponse................................139

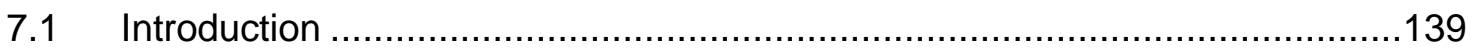

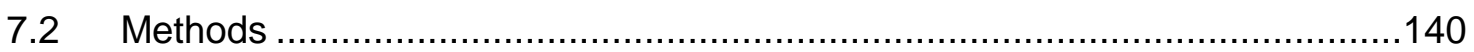

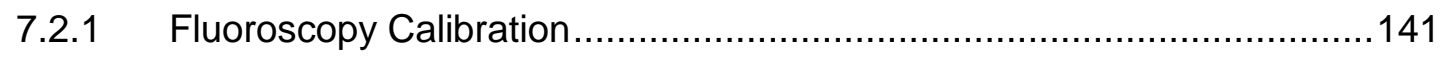

7.2.2 Registration of Fluoroscopic and CMR Images................................... 144

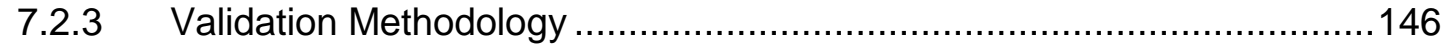

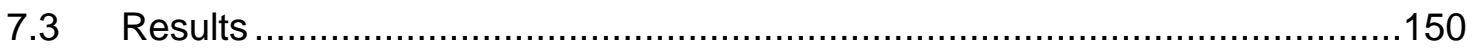

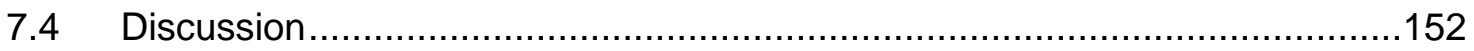

7.4.1 Advantages of Coregistration Method.......................................... 152

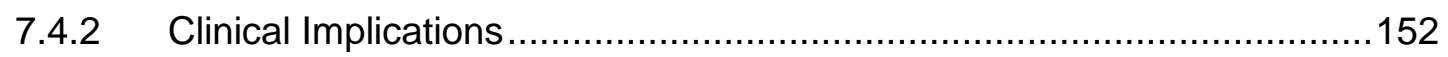

7.4.3 Design of Coregistration Method …............................................ 153

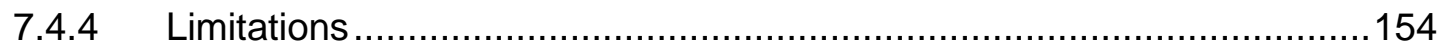

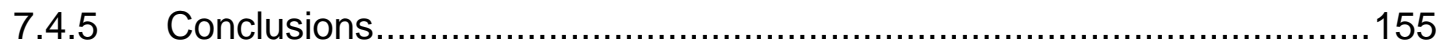

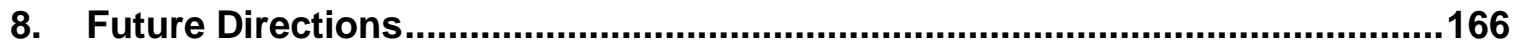


8.1 Demand Ischemia Border Zone Mechanics ........................................166

8.2 Clinical Quantitative Wall Motion Analysis ............................................168

8.3 Quantitative Wall Motion Analysis of Infarction .....................................169

8.4 Application of Lead Mapping to Evaluation of Cardiac Resynchronization

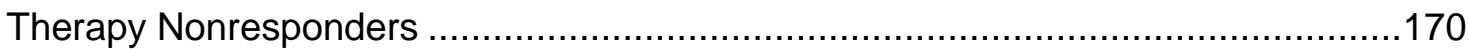

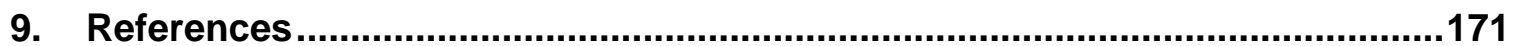




\section{List of Figures}

Figure 2.1 Experimental setup of the canine open-chest experiment...........................33

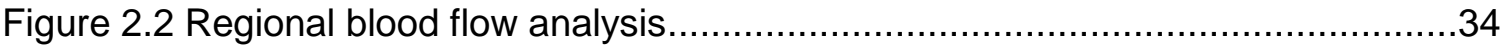

Figure 2.3 Wall motion analysis methods ……….......................................................

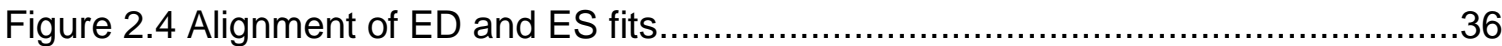

Figure 2.5 Average LV volume measured at baseline for each user .............................37

Figure 2.6 Bland-Altman analysis of EF intraobserver and interobserver variability ........38

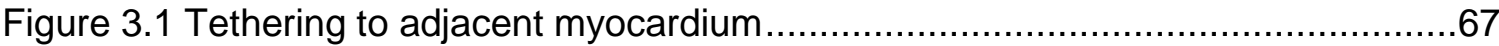

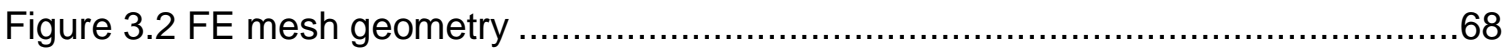

Figure 3.3 Validation of model global hemodynamic results .......................................69

Figure 3.4 Validation of model regional mechanics.................................................

Figure 3.5 Mechanical measures vs. ischemic force generation ....................................71

Figure 3.6 Measure sensitivity to ischemic force generation and size ...........................72

Figure 3.7 Size of strain abnormalies ................................................................

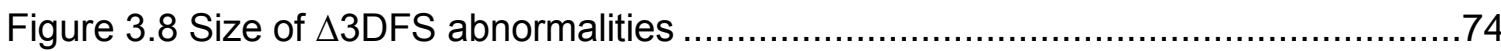

Figure 3.9 Detailed view of border zone geometry ...................................................75

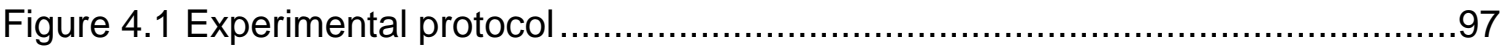

Figure 4.2 Average 3DFS during peak stress CTRL experiments..............................98

Figure 4.3 ROC analysis of 3DFS normalization methods ........................................99

Figure 4.4 Measuring PD size with $\triangle$ 3DFS ....................................................100

Figure 4.5 ROC analysis for detecting critical stenosis ...........................................101

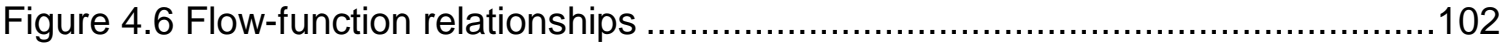

Figure 5.1 Illustration of dyskinesia severity .......................................................118

Figure 5.2 Hammer maps of 3DFS in FE models of acutely ischemic regions and healed

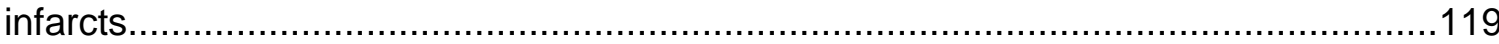


Figure 5.3 WMA size and DSI in canines during acute ischemia, 48 hours, and 8 weeks

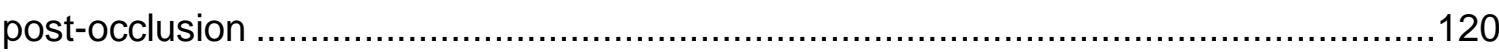

Figure 5.4 3DFS during peak stress in infarcts and demand ischemia......................122

Figure 5.5 Myocardial stiffness in infarcts and demand ischemia.............................123

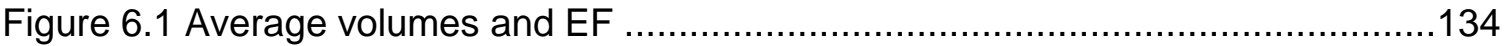

Figure 6.2 Comparison of quantitative and visually-estimated EF ...........................135

Figure 6.3 Average and standard deviation 3DFS at rest and stress ......................136

Figure 6.4 Regions of significantly different 3DFS between rest and stress ...............137

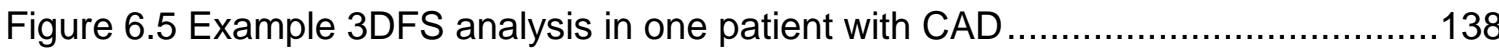

Figure 7.1 : Process flow diagram for registering fluoroscopic images to CMR images.

158

Figure 7.2 Calibration to transform 2D fluoroscopic data to 3D coordinates................159

Figure 7.3 Fluoroscopic magnification adjustment ............................................160

Figure 7.4 Canine validation of lead localization algorithm.......................................162

Figure 7.5 Validation of lead localization algorithm based on Cardiac Computed

Tomography

Figure 7.6 Validation of lead localization algorithm based on CMR venography of the

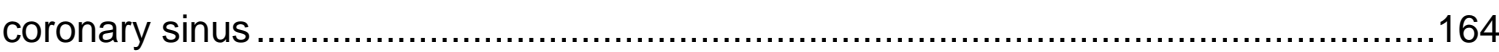

Figure 7.7 Integrated map of LV lead position and myocardial scar ..........................165 


\section{List of Tables}

Table 2.1 Intra- and interobserver variability of segmentation volumes.

Table 2.2 Linear regression coefficients relating ischemic region size to 3DFS

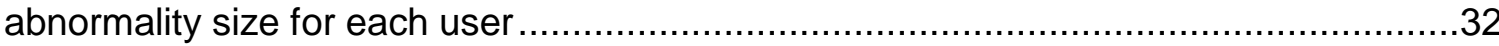

Table 3.1 Model parameters .........................................................................63

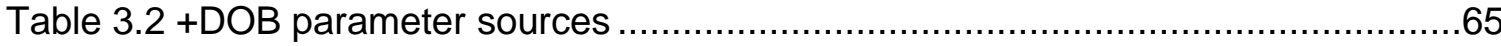

Table 3.3 +DOB parameters, model vs. data ........................................................66

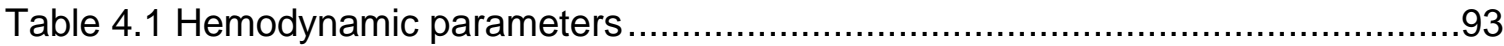

Table 4.2 ROC characteristics of measures of regional mechanics ............................94

Table 4.3 Linear regression parameters and significance ..........................................95

Table 4.4 Monte Carlo simulation of speckle tracking strain performance......................96

Table 5.1 Average experimental data for each timepoint ..........................................117

Table 7.1 Coregistration validation data summary ………………......................157 


\section{Acknowledgements}

I would first like to thank my advisor, Dr. Jeffrey Holmes, for being an excellent teacher and mentor. I am so grateful for your patience, wise advice, technical understanding, and scientific optimism through the highs and lows of scientific research. Thank you for teaching me how to be a scientist of detail and integrity. And, thank you for bringing me to the University of Virginia and Charlottesville, which I have absolutely loved calling home for the past six years.

To my doctoral dissertation committee, Dr. John Dent, Dr. Brent French, Dr. Fred Epstein, and Dr. Jeff Saucerman, thank you for excellent advice and feedback throughout my graduate studies. Thank you to Dr. Ken Bilchick for your ideas and enthusiasm, which made for an exciting collaboration that contributed greatly to this dissertation. I would also like to thank Craig Goodman, Dr. David Glover, Dr. Roy Kerckhoffs (UC San Diego), and Jeff Van Dorn (UC San Diego) for technical assistance which was essential for completing this work.

I have thoroughly enjoyed working with my colleagues and friends in the Cardiac Biomechanics Group: Dr. Susan Herz, Dr. Chris Ingrassia, Dr. Greg Fomovsky, Dr. Charlie Haggart, Dr. Andrew Rouillard, Christian Moyer, Jennie Bartell, Tracy Burcin, Dr. Beth Ames, Sam Clarke, Dr. Will Richardson, Ethan Bunting, and Alex Clark. I am so lucky to work with such smart, helpful, and fun people!

My family and friends have been so supportive and encouraging throughout my time in graduate school. Thank you to my amazing parents, Lisa Marshall and Patric Parker, and my wonderful grandmother Florence Root for your love, wisdom, pep talks, and friendship. Thank you for learning a lot of science just to ask me how my research is going each week! I am so grateful that my sister Laura joined me in Charlottesville. Thank you for being my roommate, football buddy, and kindred spirit. Thank you to my friends in Charlottesville who have been my family for the past six years. I have formed friendships with the most loving, thoughtful, and fun women during grad school, and I know they will continue to be a blessing for the rest of my life.

Finally, this research was completed through the grace of God and a lot of prayer. It is such a privilege to study the inner workings of the human body alongside its Creator.

"Now to him who is able to do immeasurably more than all we ask or imagine, according to his power that is at work within us, to him be glory in the church and in Christ Jesus throughout all generations, for ever and ever! Amen."-Ephesians 3:20-21 


\section{Background and Significance}

Coronary artery disease (CAD) is a major and increasing health risk in the United States. Identification of treatable CAD has improved with the development of imaging techniques for use during stress testing that show cardiac mechanical function, perfusion, and structure. However, with the proliferation of information available, a better understanding of the behavior of normal and diseased myocardium is needed to fully capture the diagnostic capabilities of these new technologies. The goal of this work is to improve methods for identifying vulnerable CAD from dobutamine stress testing.

\subsection{Coronary Artery Disease}

CAD occurs when plaque builds up inside the coronary arteries, limiting blood flow to the myocardium. Unstable plaques may rupture and cause myocardial infarction (MI), or heart attack. New medical and interventional treatments of CAD for prevention of MI have contributed to a decrease in the death rate from CAD in recent years, placing an even greater emphasis on identifying disease that would benefit from treatment. Stress testing in conjunction with imaging of myocardial perfusion and function is typically used to try to detect stenoses which may cause future MI. Outcomes of CAD have improved, but much work remains to identify at-risk patients while limiting unnecessary procedures.

\subsubsection{Impact}

It is estimated that 16.3 million American adults suffered from CAD in 2012. It causes 1 in 6 deaths in the United States, and is the leading cause of death for both men and 
women. ${ }^{1}$ When $\mathrm{CAD}$ leads to a $\mathrm{MI}$ this causes an estimated 16.6 years of life lost. CAD was the most expensive condition treated in 2004, due to 1.2 million hospital stays resulting in $>\$ 44$ billion in expenses. The estimated direct cost, including hospital visits and medication, and indirect cost such as loss of productivity due to CAD in 2008 was $\$ 190$ billion. $^{1}$

While CAD remains very prevalent, its mortality rate peaked in 1968 and has since decreased. Overall death rates decreased by 59\% from 1950 to 1999 , and by 26.3\% from 1997 to $2007 .{ }^{1}$ Using a detailed statistical model, it is estimated that approximately $47 \%$ of the decrease from 1980 to 2000 was due to improved treatment in this time period, while $44 \%$ was attributed to changes in population risk factors. ${ }^{2}$ Preventative medical therapies after MI, such as aspirin, beta-blockers, ACE inhibitors, and statins, accounted for $11 \%$ of the decrease in death rate, while initial treatments for MI or unstable angina such as resuscitation, medical therapy, angioplasty or coronary artery bypass grafting accounted for $10 \%$ and heart failure treatments accounted for $9 \%$. While these treatments are clearly very successful, they also leave MI survivors with damaged myocardium and more susceptible to another MI, sudden death, angina, heart failure, and stroke.

\subsubsection{Treatment}

Patients with CAD are generally classified as having either acute coronary syndrome or stable coronary disease. Patients with acute coronary syndrome experience sudden chest pain, shortness of breath, and nausea, and are urgently treated with optimal medical therapy or revascularization depending on their symptoms and risk factors. ${ }^{3}$ Stable coronary disease may be treated with the goal of preventing future cardiac 
events. Treatment usually starts with optimal medical therapy, including both drugs and lifestyle changes, but also often includes elective percutaneous coronary intervention $(\mathrm{PCl})$. During $\mathrm{PCl}$ a deflated balloon is fed through a catheter into the stenotic coronary artery using X-ray guidance, and the balloon is inflated to open the artery. Often a stent is placed to permanently open an artery. Over 1.1 million inpatient $\mathrm{PCl}$ procedures were performed in the US in 2009, ${ }^{1}$ and registry data suggests that over $85 \%$ of $\mathrm{PCl}$ procedures are elective. ${ }^{4}$ However, in a large study of 3,000 patients with inducible ischemia and $70 \%$ stenosis, meaning $70 \%$ of the normal artery diameter is blocked by the stenosis, the addition of $\mathrm{PCl}$ to optimal medical therapy did not improve outcomes over medical therapy alone..$^{5}$ Analysis of a subset of this study suggested that $\mathrm{PCI}$ is more effective in reducing ischemic burden and improving event-free outcomes in the patients that had a larger initial ischemic burden. ${ }^{6,7}$ These data suggest that improving methods for identifying patients who would benefit from $\mathrm{PCl}$ could prevent unnecessary procedures and decrease cost.

\subsection{Diagnosis}

There are many methods for determining the extent of CAD and risk of a future cardiac event in patients with stable CAD. Noninvasive imaging tests are mostly used for risk assessment, along with symptoms and lifestyle risk factors. If a patient's risk is high enough and they are referred for an invasive coronary angiogram, revascularization is completed depending on the number and severity of significant stenoses. ${ }^{8}$ Refining this diagnostic process could improve the effectiveness of revascularization procedures. 


\subsubsection{Coronary Angiography}

Coronary angiography is the gold standard method for evaluating stenosis severity and the need for revascularization. In this test, a radiopaque contrast agent is injected into the artery from a catheter, and the diameter of the stenosis is measured with X-ray fluoroscopy imaging. Gould et al. originally showed in 1974 that coronary flow and hyperemic response remain stable with increasing lesion diameter until the stenosis blocks about $70 \%$ of the artery, ${ }^{9}$ and $70 \%$ diameter reduction has become the standard clinical definition of a critical stenosis. When angiography is used to classify CAD into 1-, 2-, or 3-vessel disease and measure the diameter of stenoses, the 5-year survival rate varies widely between groups. ${ }^{8}$ However, when angiography was used as the main criteria for revascularization in the COURAGE clinical trial, no long-term benefit over optimal medical therapy was exhibited. ${ }^{5}$ It has been suggested that this limitation is likely because angiography does not measure the amount of flow restriction and ischemia that is actually experienced by the myocardium.

Functional angiography has recently been developed to measure the degree of flow restriction through the stenosis, or fractional flow reserve (FFR). FFR is defined as the maximal blood flow through a stenosis divided by the maximum theoretical flow, and is can be estimated by calculating the ratio of coronary pressure distal to the stenosis to the aortic pressure. ${ }^{10}$ In the FAME clinical trial comparing two groups undergoing PCl, the group in which stenoses with FFR $<0.8$ were revascularized showed a marked reduction in cardiac events over one year in comparison to the group treated according to stenosis anatomy measured by angiography. ${ }^{7}$ This study found that of lesions with $50 \%$ to $70 \%$ stenosis, only $35 \%$ were functionally significant (FFR $<0.8$ ), and of lesions with $70 \%$ to $90 \%$ stenosis still only $80 \%$ were functionally significant. ${ }^{11}$ Because of this convincing data, FFR is becoming widely used in clinical catheterization labs. However, 
about $30 \%$ of patients in each group still experienced angina at 1-year follow-up, indicating a significant subset of patients who were still experiencing symptoms after treatment. While coronary angiography is the current gold standard for evaluating CAD, these mixed results from the COURAGE and FAME trials have led many clinicians to argue for measurements of myocardial ischemia to be the criteria for revascularization instead of measurements of coronary anatomy. ${ }^{12}$

\subsubsection{Stress Imaging Tests}

One common method for measuring the ischemic burden caused by CAD is the cardiac stress test. Stress testing is noninvasive and safe, and therefore is often used to screen patients for coronary stenosis before angiography. Stress tests are categorized as either functional stress tests or perfusion stress tests. During perfusion stress imaging, the patient exercises or is administered a vasodilating drug such as adenosine to increase myocardial blood flow. The heart is imaged at peak perfusion with either single-photon emission computed tomography (SPECT) imaging or with contrast-enhanced echocardiography or CMR to view relative myocardial blood flow. A test is read as positive if regions of abnormal flow during vasodilation, usually caused by coronary stenosis, are apparent in comparison to the rest of the LV. Functional stress imaging is usually completed with echocardiography and occasionally CMR. Stress is induced by treadmill exercise if possible, or with a pharmacologic agent (usually dobutamine, a $\beta$ adrenergic agonist) to increase heart rate. Myocardium with sufficient coronary flow reserve increases blood flow and oxygen delivery to match increased demand, while myocardium with insufficient flow reserve, usually caused by coronary stenosis, experiences an imbalance between oxygen supply and demand known as demand 
ischemia. This causes altered mechanical and electrical behavior often identified using cardiac imaging. The mechanical movement of the LV wall, characterized clinically as "wall motion", is evaluated during stress to identify abnormally-moving ischemic regions.

Both functional and perfusion stress testing have advantages and drawbacks, and choice of test often depends on the expertise of an individual center. Meta-analysis of available studies shows that SPECT is generally more sensitive for detecting coronary stenosis with coronary angiography as the reference standard, while echocardiography is more specific. ${ }^{13}$ Perfusion imaging can often detect ischemia caused by a stenosis that is not significant enough for revascularization, decreasing its specificity. On the other hand, while wall motion abnormalities (WMAs) detected from an echocardiogram during functional stress testing often indicate a critical stenosis, the test's sensitivity is lower because sometimes a critical stenosis does not cause a visible WMA.

Current methods for detecting WMAs from echocardiography could be improved for better sensitivity to moderately ischemic regions. Dobutamine stress echocardiography (DSE) using two-dimensional echo (2DE) has been shown to have sensitivity, specificity and accuracy of $80 \%, 84 \%$, and $81 \%$ respectively in comparison to coronary angiography, ${ }^{14}$ but in a comparison of image interpretation at five centers, four of the five centers agreed in only $73 \%$ of patients. ${ }^{15}$ This relatively low interobserver agreement is attributed to the qualitative nature of diagnosis and its reliance on the expertise of the cardiologist. ${ }^{16}$ DSE can also be performed using three-dimensional echocardiography (3DE), which captures a pyramidal volume image of the entire LV. ${ }^{17}$ DSE using 3DE has been shown to be diagnostically equivalent to 2DE with reduced acquisition time,${ }^{18}$ although lower quality images and lack of single beat acquisition has so far prevented widespread clinical acceptance. ${ }^{19}$ 
One complicating factor in the specificity of stress testing is the prevalence of previous $\mathrm{MI}$ or previously unrecognized $\mathrm{MI}$ in the patient population. It is estimated that $20 \%$ of Mls in both middle-aged ${ }^{20}$ and elderly ${ }^{21}$ patients are unrecognized, and the patient population with unrecognized $\mathrm{Ml}$ is at a significantly higher risk for future cardiac events. $^{22}$ Current guidelines state that normally-perfused regions are expected to increase in function and velocity of contraction at stress compared with rest, whereas regions with a decrease in function or velocity of contraction at stress compared with low dose are considered WMAs. ${ }^{23}$ If a WMA is present at rest and does not worsen during stress, it is assumed to indicate a "fixed" WMA that indicates scar untreatable by revascularization. However, these WMAs caused by old infarcts may either falsely appear to indicate treatable ischemia or mask the presence of viable myocardium. ${ }^{24}$ The specificity of DSE may be improved if better measures to detect induced demand ischemia, even in the presence of WMAs caused by scar, are developed.

\subsection{Mechanics of Regional Ischemia}

To better understand the behavior of WMAs during stress testing, it is important to understand the factors that contribute to altered regional function. WMAs during stress testing indicate increased inotropic state without increased blood flow, or demand ischemia; however, most research on the myocardial response to ischemia has focused on decreased blood flow without increased inotropic state, or supply ischemia.

\subsubsection{Supply Ischemia}

Systolic bulging during coronary occlusion, indicating altered force generation in ischemic myocardium, was first measured by Tennant and Wiggers in $1935,{ }^{25}$ and 
further research proved that reduced blood flow and oxygen delivery causes lactate buildup, causing cytosolic acidosis in the myocyte ${ }^{26-28}$ and consequently decreased force generation. ${ }^{29}$ This acidosis, observed 1-2 minutes after the start of ischemia and continuing for 10-20 minutes, ${ }^{30}$ decreases myofibrillar sensitivity to $\mathrm{Ca}^{2+}$ and increases the magnitude of the $\mathrm{Ca}^{2+}$ transient. ${ }^{31} \mathrm{Ca}^{2+}$ binds troponin $\mathrm{C}$, which inhibits the actinmyosin crossbridge formation that drives muscle force generation in its unbound state but allows crossbridge formation when bound to $\mathrm{Ca}^{2+}$, and as a result crossbridge cycling and muscle contraction increase with increased $\mathrm{Ca}^{2+}$. As blood flow decreases from normal levels, the degree of acidosis and degree of force reduction has been shown to increase exponentially. ${ }^{32,33}$ Detailed measurement of strain through the thickness of the LV wall revealed that while circumferential and radial strains are much greater in magnitude in the endocardium than in the epicardium in normal myocardium, during acute ischemia strain does not vary transmurally very much; therefore, strain in the endocardium changes more during acute ischemia than in the epicardium. ${ }^{34}$

Finite element (FE) modeling has been useful for investigating the consequences of supply ischemia for ventricular mechanics. The effect of ischemic region size and severity of force reduction on volumetric ventricular behavior was first studied in a simple model by Bogen et $a l^{35}$ Detailed FE models that integrate the active and passive properties of myocardium with in vivo ventricular geometry and circulatory boundary conditions have since been developed ${ }^{36,37}$ and have been used to reproduce regional strain behavior during ischemia. ${ }^{38,39}$ In these models, ischemia is described with reduced or zero active force generation in the affected region, in accordance with experimental observations in ex vivo myocardium. FE modeling has proven useful in furthering understanding of the behavior of supply ischemia by separately analyzing the effects of 
ischemic region geometry, ventricular pressure, transmurality of active force reduction, and border zone active force generation on regional and global function. ${ }^{38,40-42}$

\subsubsection{Demand Ischemia}

Regional function during demand ischemia differs from supply ischemia not only due to differences in force generation within the affected region, but also because of increased force generation in the surrounding normally-perfused myocardium. Increased inotropic state is often induced with dobutamine, a cardioselective $\beta$-agonist that increases cardiac output while limiting the change in vascular resistance and heart rate caused by other inotropic agents. ${ }^{43}$ Stimulation of $\beta$-adrenergic receptors causes phosphorylation of several proteins involved in excitation-contraction coupling, resulting in increased amplitude of the cytosolic $\mathrm{Ca}^{2+}$ transient and slightly decreased $\mathrm{Ca}^{2+}$ sensitivity. ${ }^{44,45}$ These changes cause an increase in contraction, increasing ATP use, oxygen demand, and blood flow to the myocyte. Though it often occurs clinically, demand ischemia has not been studied as often in literature as supply ischemia. In a region with moderate supply ischemia, dobutamine was found to increase work performed by the myocardium. ${ }^{46}$ Another study examined tissue sections taken from dogs which underwent either coronary occlusion or severe demand ischemia, where the stenosis caused a WMA at rest and then demand ischemia was induced with pacing. In sections with equal blood flow, ATP content was higher in demand ischemia than in supply ischemia, ${ }^{47}$ indicating a difference in energy metabolism between demand and supply ischemia even at equal levels of oxygen consumption. However, this difference in energy metabolism has not been studied in depth, and the interaction between mechanisms that change contraction in demand ischemia and increased inotropic state 
has not been studied to our knowledge. Most research on the mechanical function of regions of demand ischemia has been performed clinically using imaging to measure perfusion and regional deformation during stress testing.

\subsection{Measurement of Mechanics With Imaging}

Imaging and image analysis technology have improved significantly in recent years. Clinical imaging is now used to not only visualize anatomic structures, but measure tissue motion and deformation, fluid flow velocity, tissue material properties, and even cell metabolic function. As a result, more detailed and quantifiable information about cardiac motion and deformation is now available. Much work remains to determine how to best harness this additional information for improving diagnosis of high-risk stenoses during cardiac stress testing.

\subsubsection{Cardiac Magnetic Resonance}

Although it is more expensive and less commonly-used than other modalities, CMR is the gold-standard imaging modality for measuring cardiac structure and function with high spatial and temporal resolution. As it was the first fully three-dimensional (3D) modality, stacks of cine CMR images have long been used to measure ventricular volumes and ejection fraction. ${ }^{48}$ For regional function, myocardial strain can be measured with tissue tagging in $\mathrm{CMR}^{49,50}$ although its use beyond research has been limited by time-intensive analysis. Recently, displacement encoding with stimulated echoes (DENSE) has allowed easier measurement of regional strain with high spatial resolution. ${ }^{51,52}$ With $3 \mathrm{D}$ cine DENSE, myocardial geometry and strain can be measured clinically with excellent resolution and accuracy. ${ }^{53} \mathrm{CMR}$ is often used during dobutamine 
stress testing as well, and accurately measures volumetric function ${ }^{54}$ and $\operatorname{strain}^{55,56}$ at peak doses. CMR dobutamine stress was actually found to be more effective than echocardiography for identifying critical stenoses clinically. ${ }^{57}$ Nevertheless, CMR has not become the primary modality for stress testing at most hospitals, partly due to cost, contra-indications in obese patients or those with devices, or lack of technical expertise at many centers.

\subsubsection{Echocardiography}

While it has lower spatial resolution than CMR, echocardiography is the oldest and least expensive and therefore the most widely-used modality for cardiac imaging. In recent years, many different measures of myocardial mechanics have been developed for 2DE for use during stress testing, such as fractional shortening, segmental area change, or color kinesis. ${ }^{58-60}$ However, all of these methods are limited by the selection of the center axis; measurements using a fixed centroid are affected by translational motion of the heart, while a floating centroid is biased by regional functional abnormalities and therefore can underestimate wall motion abnormalities. ${ }^{61}$ The centroid definition problem continues to limit any measure of wall motion from 2DE. Techniques for measuring strain and strain rate in the myocardium from 2DE such as tissue Doppler ${ }^{62}$ and speckle tracking have recently been developed. ${ }^{63,64}$ These methods have been shown to detect critical stenosis with moderate efficacy, ${ }^{65,66}$ and strain rate measured from speckle tracking was shown to detect CAD during DSE better than tissue Doppler. ${ }^{67}$ However, both measures are affected by image acquisition angle and through-plane motion, limiting comparison of these measures between patients. Because of these issues, no 
quantitative measurement is currently considered ready for routine clinical use for diagnosis of CAD during DSE. ${ }^{68}$

The use of 3DE for quantitative wall motion or strain measurement may solve many of these limitations. Through-plane motion is not a factor in $3 D E$, and reference frames for strain or wall motion measurement can be based on anatomical reference points instead of acquisition angle. As 3DE has only recently become widely available, the development of quantitative measures of these images is an active area of research. Measures of regional volume or area change,${ }^{69-71}$ radial fractional shortening, ${ }^{72}$ shape indices, ${ }^{73}$ and strain from 3D speckle tracking ${ }^{74-76}$ have been recently developed for 3DE. Our lab has developed a 3D measure of wall motion, three-dimensional fractional shortening (3DFS), which can accurately detect the size and location of a region with acute supply ischemia. ${ }^{77}$ While these new analysis methods show promise for increasing the amount of information available from 3DE, there is limited information on how well each measure actually detects demand ischemia. The efficacy of each measure for detecting disease must be evaluated clinically before this research is adopted into clinical use.

\subsection{Areas For Improvement}

Much can be done to improve the treatment of at-risk CAD. The definition of a stenosis which requires revascularization requires further refinement to prevent future $\mathrm{Ml}$ while minimizing unnecessary procedures. This will likely require more research linking ischemic burden, often measured with imaging, to clinical outcomes. While this is a major area of clinical research, as engineers we can improve the quality of information available for refining this definition of a treatable stenosis. Much of the information 
available from stress test images is likely underutilized for clinical diagnosis. While three dimensions of quantitative data are currently available, this data is usually only used to diagnose visually. In addition, the quantitative measurements available on clinically-used imaging machines have often not been validated for diagnostic efficacy. A better understanding of the mechanical consequences of demand ischemia would enable more strategic use of available mechanical measures from imaging. Better measures of regional mechanics may also be useful for distinguishing new demand ischemia from old infarcts. Our lab previously used a finite element (FE) model of the heart in a similar way, to test the ability of various measures to detect coronary occlusion ${ }^{78}$ and LV dyssynchrony. ${ }^{79}$ A similar method will be employed in this dissertation to measure the sensitivity of various measures to demand ischemia, and then test these measures in vivo. The goal of this work is to develop a more quantitative and empirical method for stress test interpretation, increasing the understanding of regional mechanical behavior during demand ischemia, and improving its diagnostic utility of the cardiac stress test for diagnosing CAD. 


\section{Measuring Cardiac Wall Motion From Noninvasive Imaging}

The work in this dissertation relies on techniques for quantifying LV wall motion previously developed in this lab. ${ }^{78,80}$ These methods are implemented in this dissertation to analyze 3DE images from a study of DSE in open-chest dogs in Chapter 4, CMR images of dogs with chronic infarcts in Chapter 5, and 3DE images of patients undergoing clinical dobutamine stress testing in Chapter 6. In addition, wall motion analysis is performed on a finite element model of the heart in Chapter 3, and these fitting methods are used to provide an anatomic reference for image registration in Chapter 7. Methods for performing an open-chest dog study of dobutamine stress to measure wall motion and strain will be described, as well as general wall motion analysis methods and a study of intraobserver and interobserver variability of manual endocardial segmentation of 3DE images.

\subsection{Canine Model of Dobutamine Stress}

To test quantitative wall motion analysis for use during stress testing, a model of dobutamine stress in open-chest dogs was implemented using experimental methods developed by our collaborators. ${ }^{81}$ Strain and wall motion were compared to the perfusion defect size, location, and severity measured with radiolabeled microspheres. The goal of this study, detailed in Chapter 4, was to evaluate the effectiveness of 3DFS for detecting critical stenoses during DSE, and to compare 3DFS to strain in the ischemic region measured by sonomicrometry, a gold-standard measure of myocardial deformation. 


\subsubsection{Surgical Preparation}

This experimental protocol was approved by the Institutional Animal Care and use Committee at the University of Virginia. Eleven adult mongrel dogs of both sexes (body weight $21.7 \pm 1.5 \mathrm{~kg}$ ) were anesthetized with $0.5 \mathrm{mg}$ fentanyl citrate and $40 \mathrm{mg}$ etomidate, intubated, and ventilated with room air. Anesthesia was maintained using $1.5-2.5 \%$ isoflurane gas. The left femoral vein was cannulated with an $8 \mathrm{~F}$ catheter for administration of fluids, the left femoral artery was cannulated with an 8F catheter for radiolabeled microsphere reference blood withdrawal, and the left external jugular vein was cannulated with an $8 \mathrm{~F}$ catheter for administration of dobutamine.

A left thoracotomy was performed at the fifth intercostal space, and the heart was suspended in a pericardial cradle. ECG leads were placed for continuous monitoring and ultrasound gating. A flare-tipped catheter was inserted into the left atrium for the injection of radiolabeled microspheres. A Millar pressure catheter (Mikro-Tip SPC-454D, Millar Instruments, Houston, TX) was inserted through the left carotid artery into the left ventricle (LV) to measure pressure (LVP). The left anterior descending (LAD) coronary artery was isolated, a snare ligature was loosely placed proximal to the first diagonal, and instrumentation was placed as illustrated in Figure 2.1. An ultrasonic flow probe (model T-206, Transonic Systems, Ithaca, NY) was placed distal to the snare and the space between the probe and vessel wall was filled with an acoustic gel. Nine sonomicrometer crystals (2T-36S-40-RS, Sonometrics, London, Ontario, Canada) were inserted into the LV midwall using a metal introducer. Six crystals were used to measure changes in length of the three axes of the LV (anterior-posterior, septal-lateral, and base-apex), while the remaining three crystals were placed in the anterior LV wall at a depth of $7-10 \mathrm{~mm}$, in the region supplied by the LAD, to measure midwall strains in the 
circumferential-longitudinal plane. During each data acquisition, ECG, arterial pressure, LAD flow, LVP, and sonomicrometry signals were recorded. The experimental protocol, in which each dog undergoes a DSE test both with and without a critical stenosis, is explained in more detail in Chapter 4.

\subsubsection{Assessment of Functional Data and Regional Strain}

Hemodynamic data were processed using the Sonometrics software, SonoSoft ${ }^{\circledR}$, and custom MATLAB software (Mathworks, Natick, MA, release R2012a, version 7.14.0.739). Noise and level-shifting in the signals were manually removed using SonoSoft. LV volume was calculated from the apex-base, anterior-posterior, and septallateral axis lengths for the purpose of selecting the end-diastolic (ED) and end-systolic (ES) time points, using a truncated ellipse model as described previously. ${ }^{82,83}$ LAD flow was calculated for one beat by numerically integrating the flow probe signal over time using the trapz function in MATLAB, starting at ED and ending at the next ED time point. Heart rate, integrated LAD flow, and distances between each of the three crystal pairs in the LAD region at ED and ES were calculated for five consecutive beats and then averaged. Coronary flow reserve (CFR) was calculated by dividing the LAD flow measured by the flow probe during peak stress by flow measured just before dobutamine administration. All experiments with CFR $<1.1$ measured with the LAD flow probe were considered critical stenoses, and measures were evaluated on their ability to separate these critical stenoses from CTRL and STEN with CFR $>1.1$.

Strains were derived from segment lengths using methods described previously. ${ }^{34,83,84}$ The angle $\theta_{i}, i=1-3$, of each sonomicrometry pair in the LAD perfusion region with respect to the circumferential direction was measured from post-mortem 
photographs of the LV. Unit vectors $\mathbf{n}_{\mathrm{i}}$ in the direction of each segment length were computed as:

$$
\mathbf{n}_{\mathrm{i}}=\left[\cos \left(\theta_{\mathrm{i}}\right) \sin \left(\theta_{\mathrm{i}}\right)\right]
$$

The undeformed reference state $\mathrm{d}_{\mathrm{i}}$ was defined for each segment as:

$$
\mathrm{d} \mathbf{U}_{\mathrm{i}}=\mathrm{d} \mathrm{S}_{\mathrm{i}} \cdot \mathbf{n}_{\mathrm{i}}
$$

where $d S_{i}$ is the undeformed length of segment $i$. The Lagrangian finite strain tensor $\mathbf{E}$ can be computed using $\mathrm{d} \mathbf{U}$ and the deformed state $\mathrm{du}::^{85}$

$$
2 d \mathbf{U}_{i} \cdot \mathbf{E} \cdot d \mathbf{U}_{i}=d \mathbf{u}_{i} \cdot d \mathbf{u}_{i}-d \mathbf{U}_{i} \cdot d \mathbf{U}_{i}
$$

To implement Equation (3) in MATLAB, it was written in component form:

$$
2 \cdot\left[d U_{i}(1)^{2} \cdot E_{11}+2 \cdot d U_{i}(1) \cdot d U_{i}(2) \cdot E_{12}+d U_{i}(2)^{2} \cdot E_{22}\right]=\left[d s_{i}^{2}-d S_{i}^{2}\right]
$$

where $E_{11}, E_{22}$, and $E_{12}$ are the circumferential, longitudinal, and shear components, respectively, of the strain tensor $\mathbf{E}$ and $\mathrm{ds}_{\mathrm{i}}$ is the deformed length of segment $\mathrm{i}$. Equation (4) was solved in MATLAB for the components of $\mathbf{E}$ using the least squares left division operator, 'l'. Principal strains $\left(E_{1}\right.$ and $\left.E_{2}\right)$ were calculated as the eigenvalues of $\mathbf{E}$ using the 'eig' function in MATLAB, and the principal stretch ratios $\left(\lambda_{1}\right.$ and $\left.\lambda_{2}\right)$ were calculated using the equation $E_{i}=0.5^{*}\left(\lambda_{i}^{2}-1\right)$. Area strain, or the fractional change in the area of the sonomicrometer triangle $\left(A_{E S}-A_{E D}\right) / A_{E D}$, was computed as $\lambda_{1}{ }^{*} \lambda_{2}-1$.

Strain was calculated at a frequency of $195 \mathrm{~Hz}$ over the entire cardiac cycle. Strain rate in the circumferential and longitudinal directions was measured by taking the derivative of strain with respect to time. Temporal averaging of the strain rate curves was used with a window of $10 \mathrm{~ms}$. Peak systolic strain rate was calculated as the peak 
negative strain observed between the opening of the aortic valve, defined by the time after ED when LV volume begins changing, and closing of the aortic valve, or ES. This is the same strain rate parameter most often used with Doppler echocardiography to detect strain rate abnormalities. ${ }^{86-88}$

\subsubsection{Assessment of Regional Myocardial Blood Flow}

After the experiment, the atria, valve, and right ventricle tissue were removed. Images of the ventricle were taken with sonomicrometers in place for strain analysis, and then the sonomicrometers were removed. The depth of each crystal was measured, and care was taken to remove as little myocardial tissue as possible. The heart was fixed in formalin for at least 24 hours, and then removed for processing of radiolabeled microspheres. A slice through the epicardium was cut from the base to the apex in the center of the two RV insertion lines to orient each short axis slice. The LV was sectioned into eight equally-spaced slices in the longitudinal direction, and then sliced into eight circumferential pieces spaced every 45 degrees except in the most apical slice, which was split into four pieces (Figure 2.2). This resulted in 60 transmural blocks evenly distributed over the LV surface (Figure 2.2C), as used in previous experiments. ${ }^{77}$ Tissue mass and regional myocardial blood flow was determined for each block using standard methods for quantification of radioactive microspheres. ${ }^{81}$

\section{Definition of Perfusion Defect}

The myocardial blood flow measured by microspheres was used to measure the size of the perfusion defect (PD) as the fraction of tissue blocks receiving abnormal flow. As selecting a threshold for defining this abnormal flow is not straightforward, multiple potential thresholds were tested. Previous work in supply ischemia ${ }^{77}$ defined abnormal 
flow as a percent decrease from nonischemic blocks near the base. However, this was not reliable during dobutamine stress as the amount of increased blood flow was not consistent among different regions of the heart. Instead, we considered using either myocardial flow reserve $(M F R)<1.1$, defined as flow at peak stress divided by flow at rest, or fractional flow reserve $(\mathrm{FFR})<0.8$, defined as flow at peak stress with stenosis divided by flow at peak stress without stenosis. Baseline myocardial flow measured with microspheres was only measured at the start of the experiment, so MFR was evaluated both using this initial myocardial flow measurement and by normalizing this myocardial flow measurement with the ratio of coronary flow measured by the flow probe before each stress test, to approximate a rest myocardial flow measurement before the second DSE test.

For all of these measures, the mean size of the PD changed but correlation with the wall motion abnormality size did not, as detailed in Chapter 4. It seems the choice of threshold just determines how much of the border zone to include in PD size. Therefore, size of the PD was defined as the fraction of blocks in which the MFR was less than 1.1, the same as the coronary flow CFR cutoff for a critical stenosis, for simplicity and consistency.

\subsection{Quantitative Wall Motion Analysis}

The wall motion analysis process begins with segmentation of the endocardial surface from 3DE or CMR images. Anatomical landmarks are selected from the images to transform the endocardial surface data, represented by a cloud of Cartesian points, into a standardized coordinate system to orient the fit and eliminate translation or rotation 
between ED and ES. Finally, the surface data is fit with a parametric function in a prolate spheroidal coordinate system and motion measurements are calculated from this fitted surface. Some adaptations have been made to the analysis techniques since the previous work. ${ }^{77,78,80}$ These changes include new segmentation methods for CMR images, new landmark selection methods in 3DE, new methods for re-orienting the fit to account for the asymmetric geometry of the canine LV, and different smoothing constraints for fitting.

\subsubsection{Segmentation}

\section{Three-Dimensional Echocardiography}

3DE images were acquired using either a Philips 7500 (dogs) or Philips iE33 (patients) ultrasound system with a full volume, four beat acquisition. No contrast was used. Images were considered to be of adequate quality if the endocardial border was visible at both ED and ES over most of the surface, and if no stitch artifacts were visible within the LV cavity. All 3DE images were segmented using Tomtec 4D LV-Analysis ${ }^{\circledR}$ software version 2.6 (Tomtec Imaging Systems, Unterschleissheim, Germany). ED and ES frames were selected as the frame with maximum LV volume and the frame $45-90 \mathrm{~ms}$ prior to the opening of the mitral valve, respectively. The software generated a surface representing the LV endocardial border at ED and ES from manual contours of the three standard long axis views, and the user manually edited these two surfaces as necessary (Figure 2.3A). Papillary muscles were excluded, the aortic valve was traced at the valve plane, and the trace border was placed to follow the mitral valve annulus. A cloud of points representing the final endocardial surface was exported in Cartesian coordinates using the Tomtec Export Measurements feature. 
Three points at the apex, base, and septum were selected to remove translation and to anatomically orient the fit. Separate Tomtec analysis files were saved for ED and ES to select these points. For each time point, the view plane center axis, which defines the intersection of the three long axis views displayed by the segmentation software, was oriented to cut through the center of the mitral valve and through the apex. Then, a mesh was created to closely follow this center axis line and come to a point at the apex. In later analysis the point closest to this selected apex on the endocardial segmentation was used as the apical reference point. The tracing border was made to follow the mitral valve annulus, and in later analysis the centroid of this border was used as the basal reference point. Finally, the short axis cut was placed roughly $1 / 3$ of the distance between the base and apex, closer to the base, and a point on the mesh in the middle of the septum was extended out to split the two RV insertion points. This septal reference point was used to account for ventricular rotation. This mesh was exported using the Tomtec Export Measurements feature. The exported coordinates are reported in image coordinates, so when multiple segmentations are exported for the same image these data are reported in the same coordinate system. Therefore, the selected landmark coordinates can be directly used to transform the endocardial segmentation.

\section{Cardiac Magnetic Resonance}

Endocardial surface data from MR images was used in some situations to measure wall motion or to anatomically orient scar or lead location data. All images were acquired on a Siemens Avanto 1.5-T clinical scanner, and cine SSFP images were analyzed for ventricular function. The endocardium was segmented from six to ten short axis images and zero to three long axis images using either the Siemens Argus Ventricular Function tool (Siemens Healthcare, Erlangen, Germany) or the freely available software Segment 
v1.9 R2278 (http://segment.heiberg.se). ${ }^{89}$ ED and ES were chosen as the time points with the largest and smallest volumes, respectively. Papillary muscles and trabeculae were included in the cavity. Contours were exported for each slice and converted to 3D Cartesian coordinates in the scanner coordinate system using DICOM header data for further analysis.

Points representing the apex, base, and septum were selected for ED and ES. In cases with long axis cine images, the center of the mitral valve was selected on all long axis images and averaged to represent the base. The apex was selected on the long axis views, averaged, and then shifted parallel to the most apical short axis image to be located in the center of the most apical short axis segmentation. If long axis images were not available, the apex and the base were chosen as the centroid of the most apical and basal short axis slices, respectively, and then shifted normal to the short axis plane a distance of half of one slice thickness. Finally, to locate the septal landmark the short axis slice closest to $1 / 3$ of the base-apex distance away from the base was selected and the two RV insertion points were chosen. The septal landmark was chosen as the point on the endocardium bisecting the arc connecting the two insertion points.

\subsubsection{Fitting}

The exported endocardial data was analyzed using custom software written in MATLAB as described previously. ${ }^{77,78,80}$ The endocardial data were transformed into a cardiac coordinate system using the three anatomical landmarks selected at ED. This cardiac coordinate system has axes directed parallel to the base-apex axis $\left(x_{1}\right)$, through the midseptum $\left(x_{2}\right)$, and through the posterior wall $\left(x_{3}\right)$ from an origin located $1 / 3$ of the distance from the base to apex along the LV long axis. Parametric surfaces were fit through the 
data in prolate spheroidal coordinates, illustrated in Figure 2.3B. In this elliptical coordinate system, every point on the LV surface is represented by circumferential $(\theta)$ and longitudinal $(\mu)$ angles from the origin and a radial distance along an arc from the central axis $\lambda=\lambda(\mu, \theta)$ (Figure 2.3C). The focal length of the coordinate system was set to (2/3 of the base-apex distance)/cosh(1) to scale the fitting problem, and the fit was chosen by minimizing the least squares error of data points in $\lambda$.

\section{Landmark Placement}

Some adjustments were made to landmark placements to account for variations in image quality and ventricular anatomy. First, the RV insertion points were often difficult to see or cut off in the image volume, creating uncertainty in selecting the septal landmark. Therefore, we opted to use the ED septal landmark for ES as well and forego the rotation correction. This was implemented in CMR fits as well for consistency, although selecting the septal point using the clearly visible RV insertion points in CMR was very repeatable.

We also found that in canines, most of the ventricles analyzed were not symmetric around the apex-base axis; the axis was usually closer to the posterolateral wall, near $\theta=135^{\circ}$. This created two problems. First, the fits did not perform well when some walls were very close to the axis, as the least squares error is measured in the $\lambda$ direction and this distance between the point and the surface is not the minimum distance when the data represents a surface that is more parallel than perpendicular to the $\lambda$ direction. More importantly, alignment between ED and ES using an axis that is closer to one wall caused underestimation of wall motion in the near wall and overestimation in the far wall. An example of this problem is shown in Figure 2.4. In this case, the apex is closest to the posterior wall. By using the apex-base axis to align ED 
and ES, as shown in Figure 2.4A, the method introduced tilt into the ES data in comparison to Figure 2.4B, where ED and ES are not realigned relative to one another. This tilt occured because the basal landmark moved mostly in the $x 1$ direction from ED to ES, while the apex moved significantly in $x 2$ and $x 3$ as well. This tilt caused exaggerated or underestimated wall motion measurements over the entire ventricle, as shown in the 3DFS hammer maps for the aligned (Figure 2.4A) and unaligned (Figure 2.4B) data.

Two adjustments were implemented in the open-chest dog data in Chapter 4 to address these issues. First, because the transducer and gel offset were placed on the apex of the heart during the experiments, the heart did not move much from ED to ES. The origin, located at $1 / 3$ the base-apex distance on the base-apex axis, only moved $2.03 \pm 0.95 \mathrm{~mm}$ in rest control dogs and $2.71 \pm 0.87 \mathrm{~mm}$ in peak stress control dogs. Therefore, only ED landmarks were used in these experiments and no motion correction was implemented to avoid misalignment. To improve fit quality, the apical landmark, originally located at prolate spheroidal coordinates $\left(\lambda_{A}, 0^{\circ}, 0^{\circ}\right)$, was shifted to to $\left(\lambda_{A}, \mu_{\text {shift, }}\right.$, $\left.\theta_{\text {shift }}\right)$ where $\mu_{\text {shift }}$ and $\theta_{\text {shift }}$ were chosen to visually orient the axis in the center of the ED endocardial data. Values of $\theta_{\text {shift }}=315-350^{\circ}$ and $\mu_{\text {shift }}=15-25^{\circ}$ were used. After this shift was applied the data was transformed again so that this new apex defined the center axis. The result of this combination of shifted apex and no alignment of ED and ES is shown in Figure 2.4B, where the shifted apex is shown in green. These adjustments reduced the standard deviation of the 3DFS averaged over all dogs from 0.075 to 0.072 for images taken at rest, and from 0.16 to 0.15 for images taken during peak stress in the absence of a stenosis.

Element and Smoothing Optimization 
Data points were projected on lines of constant $\mu$ (ranging from 0 to $120^{\circ}$ ) and $\theta$ (ranging from 0 to $360^{\circ}$ ) onto an initial mesh of undeformed ellipsoidal bicubic Hermite elements with $\lambda=1$. For 3DE data, the Tomtec endocardial segmentation software exports 640 points evenly spaced on the endocardial surface. To ensure the presence of at least three data points in each direction per element for a cubic fit, a 16 element mesh with four elements evenly spaced in $\mu$ (longitudinal direction) and four elements evenly spaced in $\theta$ (circumferential direction) was used. CMR segmentation data was finely spaced circumferentially but had only some long axis data and six to ten short axis slices in the longitudinal direction, so an 8 element mesh with four circumferential and two longitudinal elements was used.

Smoothing was imposed in the fit by penalizing bending and stretching of the fitted surface as described by Hashima et al. ${ }^{90}$ The smoothing coefficients representing the weights penalizing stretching and bending were varied, keeping the ratio equal to 0.5 , until an increase in root mean squared error in the fit was just detectable. The optimal weights for tension coefficient $\alpha$ and bending coefficient $\beta$ were 0.01 and 0.02 , respectively, for both $3 \mathrm{DE}$ and CMR data.

\section{Quantitative Wall Motion Analysis}

Motion of the endocardial surface was quantified by calculating the three-dimensional fractional shortening (3DFS) in $\lambda$ between ED and ES $\left(3 D F S=\left(\lambda_{E D}-\lambda_{E S}\right) / \lambda_{E D}\right)$ at each location of constant $\mu$ and $\theta$ on the surface. 3DFS is displayed on a Hammer projection map (top) and a standard bulls-eye map (bottom) in Figure 2.3D. The Hammer projection preserves relative surface areas, so that the size of any region of interest on the map reflects its true size as a fraction of LV surface area; by contrast, on a bulls-eye map the basal segments appear larger than their true size, while the apical segments 
appear smaller. For this reason, Hammer maps are used for visualization in this report. All reported LV cavity volumes were computed as the volume enclosed by the fitted surface.

\subsection{Interobserver Variability of 3DE Manual Segmentation}

The major source of error in calculating 3DFS from 3DE images in this dissertation is the accuracy of endocardial segmentation. All segmentations were manually edited from an automatic initial guess, and the accuracy of the final segmentation depends on both image quality and the user. We tested the effect of user cardiology expertise on the intraobserver and interobserver variability of volumes and 3DFS measurement in highquality 3DE images acquired in open chest dogs. Using this data set, the quality and repeatability of segmentation by different users was evaluated, a gold standard user was chosen, and in the future new users can be trained until they reach the gold standard quality.

\subsubsection{Methods}

A subset of 24 images were selected from a previously reported data set of open chest dog images. ${ }^{77}$ Twelve images were acquired after two minutes of transient occlusion of either LAD or left circumflex (LCx) arteries in a proximal or distal location, and 12 were acquired during normal function immediately prior to occlusion. ED and ES time points were selected prior to analysis in each image, and the endocardium was segmented at these two time points by each user. Five users completed eight training image sets, and then traced each image (both ED and ES) twice while blinded to the image source. 
Three users were attending cardiologists considered experts in interpreting echocardiograms (labeled A1, A2, and A3), one user was a cardiology imaging fellow $(\mathrm{F} 1)$, and one user was the author, an engineering graduate student (S1). Each user completed the data analysis over a span of one to five months. All data was segmented once, and then the entire set was segmented again to ensure sufficient time between analyses for evaluation of intraobserver variability. The pre-ischemia baseline and subsequent ischemia images were always segmented at the same time.

Segmentation data from each image was transformed using previously-chosen apex, base, and septal landmarks and fit using the methods described above. No apical adjustment was necessary. LV volumes at baseline were averaged for each user and compared to other users using a paired Student's t-test. Ejection fractions (EF) from the same image were compared using Bland-Altman analysis ${ }^{91}$ between repeated segmentations for each user to assess intraobserver variability, and the second completed segmentation for each user was compared to other users to assess interobserver variability. In addition, interobserver variation in volume was calculated for each image by dividing the standard deviation of the measured LV volume from each user divided by the mean measured volume. Intraobserver variation in volume was calculated by dividing the difference in measured volume in the two repeated analyses by the average volume, and averaging this value over all images and users for ED volume and ES volume. These measures were compared to results from Mor-Avi et al. ${ }^{92}$ to test whether our observed variation was comparable to segmentation variation observed at other institutions. Finally, regional segmentation accuracy was assessed by comparing the size of a wall motion abnormality (WMA) measured by 3DFS to the size of the ischemic region measured in the dog by microsphere analysis. WMA size for 
regional supply ischemia was measured by subtracting 3DFS in the baseline image from 3DFS in the ischemic image ( $\triangle$ 3DFS) and measuring the fraction of the LV surface that displays $\triangle 3 \mathrm{DFS}<-10 \%$, as defined previously. ${ }^{77}$ This comparison to microsphere data provides a gold standard of sorts, This comparison to microsphere data tests whether the segmentation was accurate enough to detect ischemic regions.

\subsubsection{Results}

\section{Global Measures}

Total intraobserver and interobserver values for measuring volume are reported in Table 2.1. All variation values for the current study are similar to those reported in Mor-Avi et al., ${ }^{92}$ a study in which a similar 3DE semiautomatic segmentation method with manual editing was evaluated in multiple institutions. The average measured volume in the baseline images from each user is displayed in Figure 2.5. While most users had very similar values, user A1 measured volumes significantly smaller than the others.

The effect of this variation on EF is examined more closely using Bland-Altman analysis, shown in Figure 2.6A. Experienced users showed lower intraobserver agreement, as users F1 and S2 had tighter limits of agreement between repeated segmentations than $\mathrm{A} 1, \mathrm{~A} 2$, and $\mathrm{A} 3$. Analysis of interobserver variability between attending cardiologists and comparing $\mathrm{A} 3$ to $\mathrm{F} 1$ and $\mathrm{S} 2$ are shown in Figure 2.6B. We see little consensus on EF measurement among attending cardiologists. Overall, we see fairly wide limits of agreement of $\pm 15 \%$, which is extremely large as the mean measured EF was $33 \%$. 


\section{Regional Measures}

Linear regression between size of the ischemic region measured by microspheres and the WMA size with $\triangle 3 D F S$ was computed for each user, and the results are shown in Table 2.2. While the agreement of EF between users was limited, analysis by all users except A2 showed good-to-excellent ability to measure the size of an ischemic region. For the best-performing users, $\mathrm{A} 3$ and $\mathrm{S} 1$, the relationship between ischemic region size and abnormal wall motion size was very close to $1: 1$ with a very small y-intercept offset.

\subsubsection{Discussion}

In this study, all but one user was able to segment 3DE images with enough accuracy to correctly measure the size of a wall motion abnormality. However, the limits of agreement in measuring $\mathrm{EF}$, up to $20 \%$ between users, were larger than the margins used to detect disease clinically, where $\mathrm{EF}<35 \%$ is generally used as a cutoff for disease. Users with more experience reading echocardiograms did not perform better than trainees. Overall, measuring change in 3DFS with manual segmentation displayed less variability than measurement of EF and could be a very reliable measure if the user has been trained to meet a previously-established gold standard.

Why would EF have more variability than $\triangle$ 3DFS when both were measured from the same segmentations? In observing different users, it was clear that each chose a slightly different location for the endocardial border. In fact, the endocardial border can be difficult to visualize in 3DE and deciding how to include trabeculations and papillary muscles in the cavity can be difficult. ${ }^{92}$ These segmentation decisions will change results for EF. However, change in $\triangle$ 3DFS relies on detecting the difference between 3DFS in a baseline image and the ischemic image. If the user is consistent in border placement 
between images in reference to the anatomy, small differences in selecting the actual location of the border do not matter. Therefore, $\triangle 3 \mathrm{DFS}$ shows promise in this study to be a more repeatable measure of function than EF.

Although the time of each analysis was not recorded, by observing the each user it was clear that those who spent the most time on analysis had the best results for measuring WMA size. It was important to be thorough in not only the individual ED and ES segmentations, but to make sure the motion between these time points represented wall motion accurately even in locations where the border cuts off trabeculations or papillary muscles. Accurate analysis by an experienced user takes approximately 20 minutes per image, which is not feasible in a clinical environment. These results demonstrate that if 3DFS analysis is desired in a clinical situation, a technician could be trained to analyze images just as well as an expert cardiologist. Segmentations from user S1 were designated as gold-standard for training future users, as this user had the smallest intraobserver variability and best agreement with ischemic region size. All segmentations in this dissertation were performed by this user. 
Table 2.1 Intra- and interobserver variability of segmentation volumes

\begin{tabular}{lllll}
\hline & Intraobserver & \multicolumn{2}{l}{ Interobserver } \\
& EDV & ESV & EDV & ESV \\
& & & & \\
\hline Current Study & $5 \pm 5 \%$ & $6 \pm 5 \%$ & $9 \pm 2 \%$ & $12 \pm 5 \%$ \\
Mor-Avi et al, 2008 & $5 \pm 5 \%$ & $10 \pm 11 \%$ & $8 \pm 8 \%$ & $13 \pm 14 \%$ \\
& & & & \\
\hline
\end{tabular}


Table 2.2 Linear regression coefficients relating ischemic region size to 3DFS abnormality size for each user

\begin{tabular}{cccc}
\hline User & Slope & Y-Intercept & $R^{2}$ \\
\hline A1 & 1.27 & 0.00 & 0.69 \\
A2 & 0.39 & 0.22 & 0.15 \\
A3 & 1.07 & 0.03 & 0.80 \\
F1 & 0.94 & 0.12 & 0.60 \\
S1 & 1.08 & 0.06 & 0.84 \\
\hline
\end{tabular}




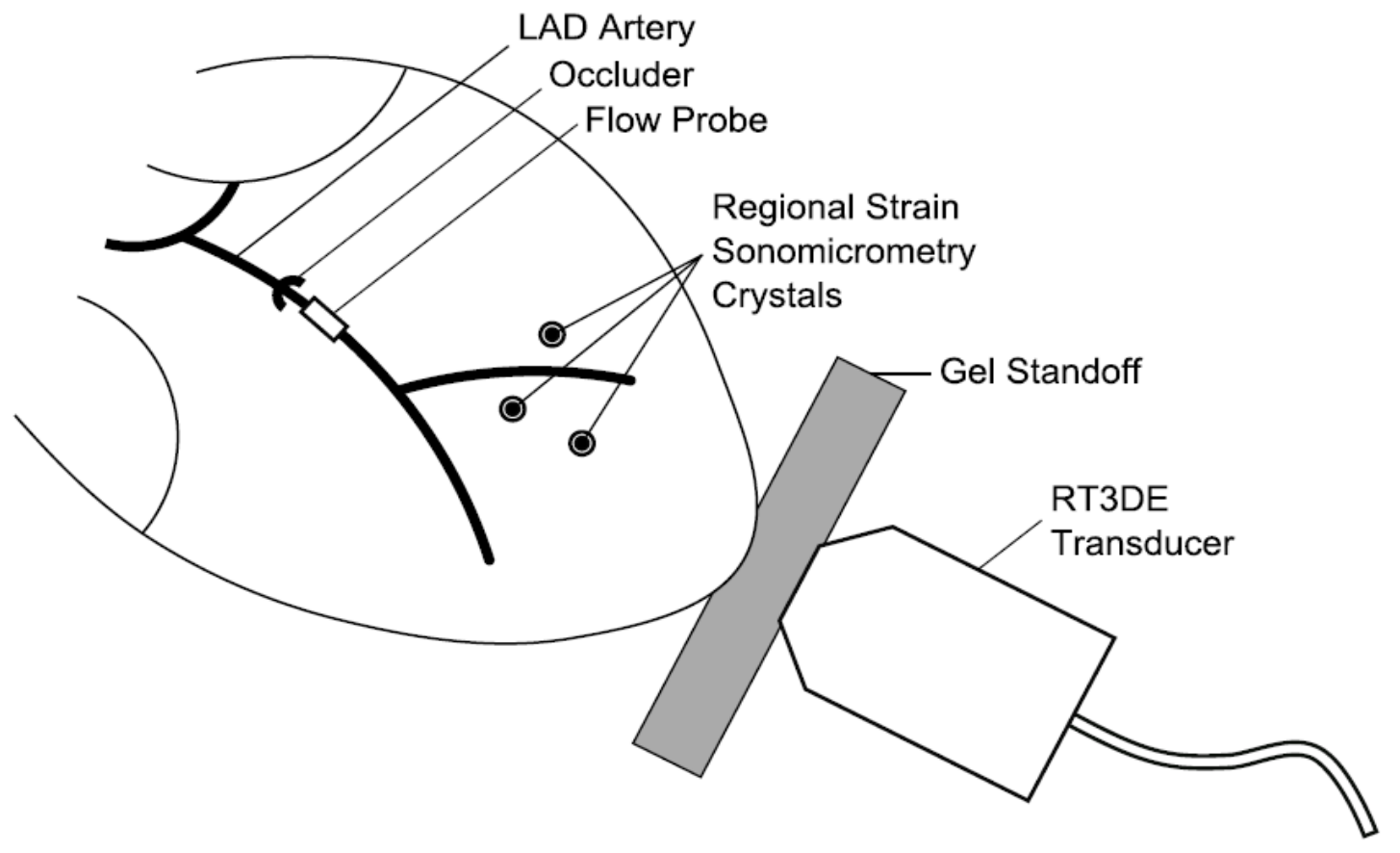

Figure 2.1 Experimental setup of the canine open-chest experiment 

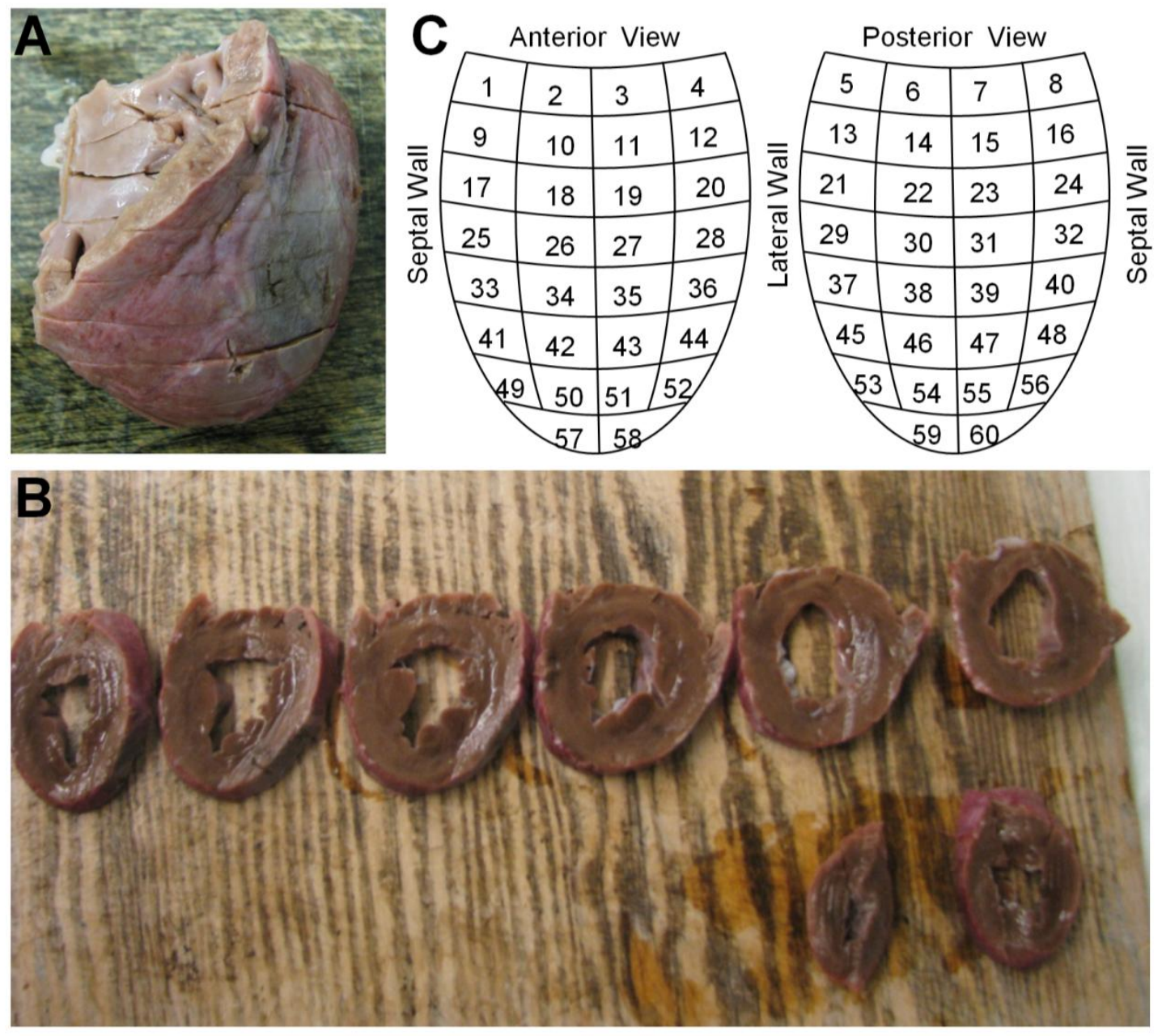

Figure 2.2 Regional blood flow analysis

The fixed LV was cut into eight equally-spaced short axis slices (A). The orientation of each slice was preserved. Each slice was cut into eight pieces, with the first cut placed between the two $R V$ insertion points (B). The orientation of each myocardial slice was used to define the size and location of the perfusion defect (C). 


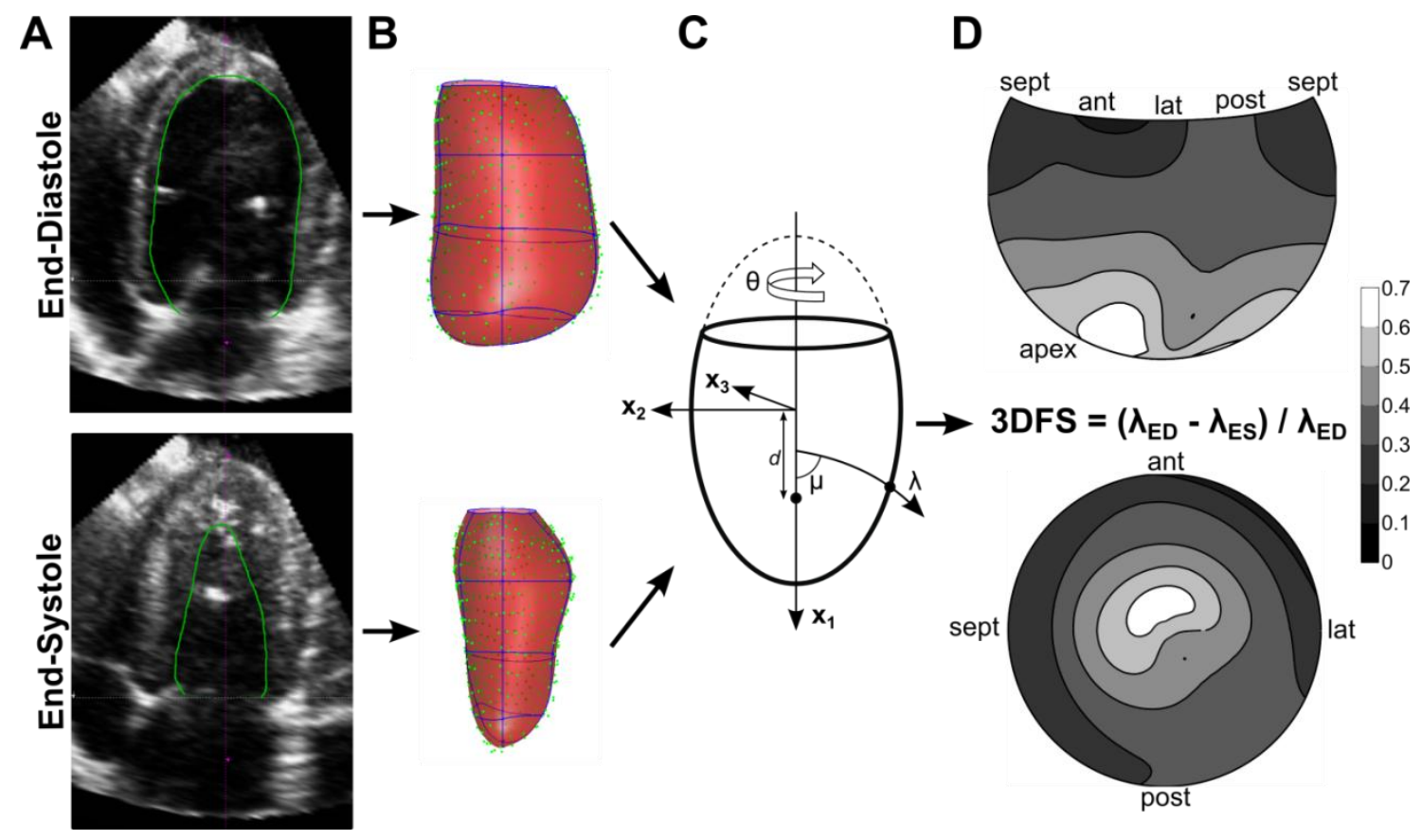

Figure 2.3 Wall motion analysis methods

Wall motion analysis methods demonstrated during CTRL peak stress. (A) Apical 4 chamber view of the 3DE image. The endocardial border was segmented (green) at enddiastole and end-systole using Tomtec 4D-LV Analysis software. (B) Data points representing the segmented surface (green) were exported and fit with a parametric surface (red, element borders blue). (C) Surfaces were fit in prolate spheroidal coordinates, diagrammed here. Cartesian coordinates $\left(x_{1}, x_{2}, x_{3}\right)$ are transformed into curvilinear coordinates $(\lambda, \mu, \theta)$, scaled by the focal length $d$. (D) Average threedimensional fractional shortening (3DFS) at peak stress during CTRL experiments is displayed on a Hammer projection map (top) and a standard bulls-eye map (bottom) representing the LV surface. 
A Unshifted Apex,
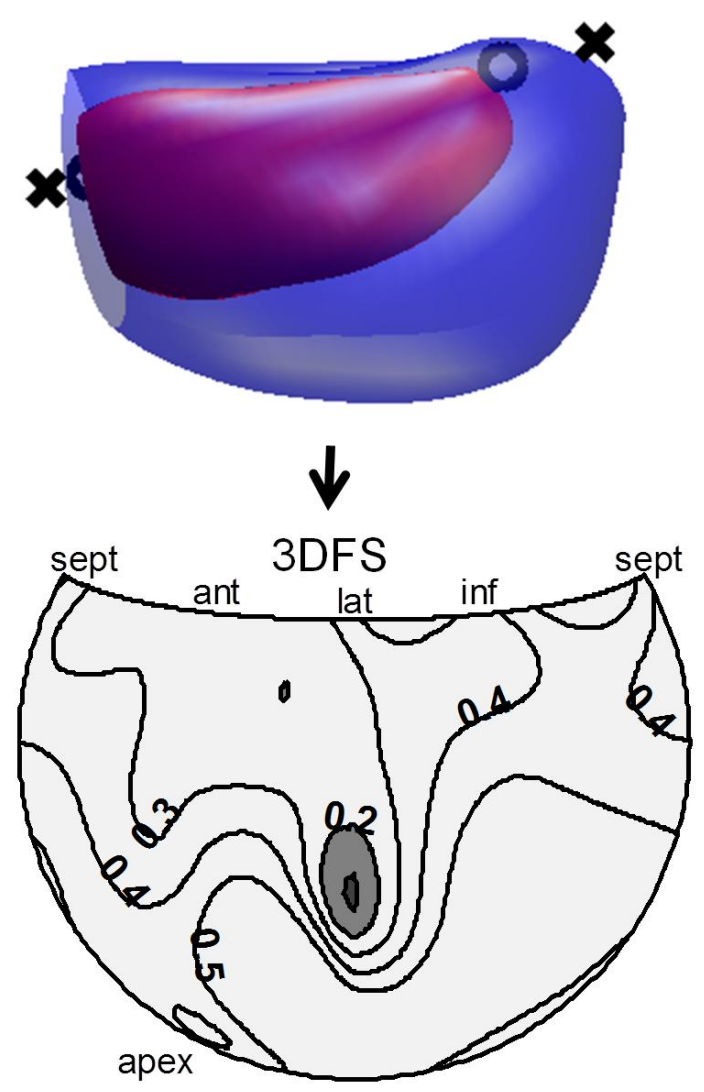

B

Shifted Apex, No ED-ES Alignment
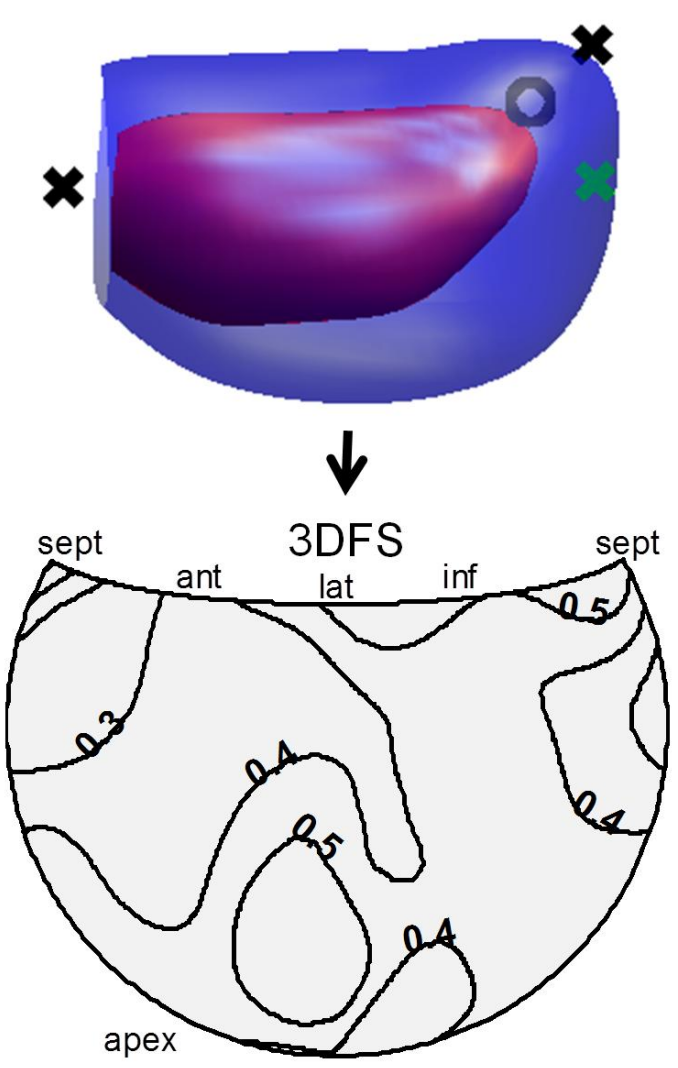

Figure 2.4 Alignment of ED and ES fits

Methods for aligning the ED (blue) and ES (red) fits were adjusted to account for the asymmetry of the canine LV. (A) Because the apex is closer to the posterior wall, aligning ED and ES using the ED and ES landmarks ( $\mathbf{X}$ and $\mathbf{O}$, respectively) causes regions of artificially elevated and depressed 3DFS. (B) Because the ventricle was held stationary during the experiment, normal inward wall motion can be measured without accounting for ventricular motion. To improve fits, the apex is shifted so that it is located more centrally in the LV (green $\mathbf{X}$ ). 


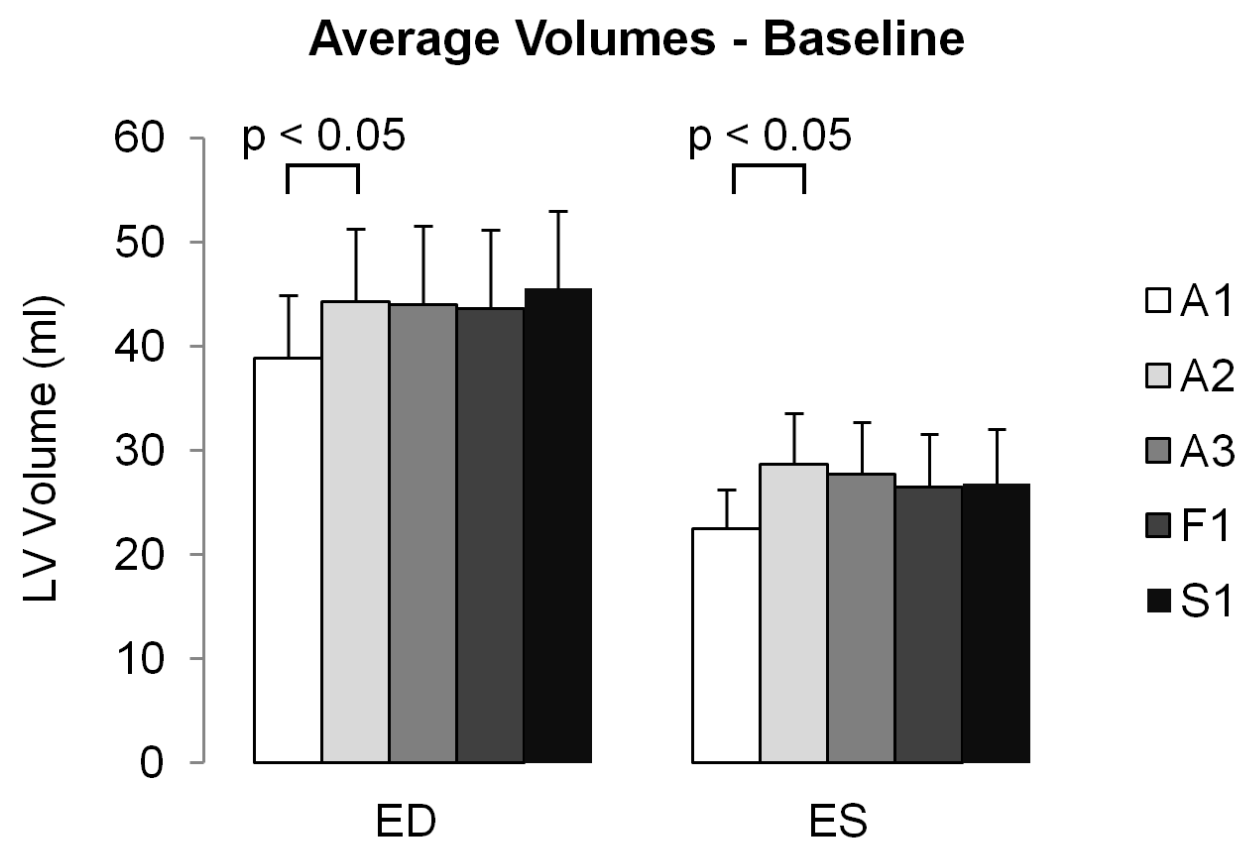

Figure 2.5 Average LV volume measured at baseline for each user

Displayed error bars are +1 standard deviation. 
Intraobserver variability:

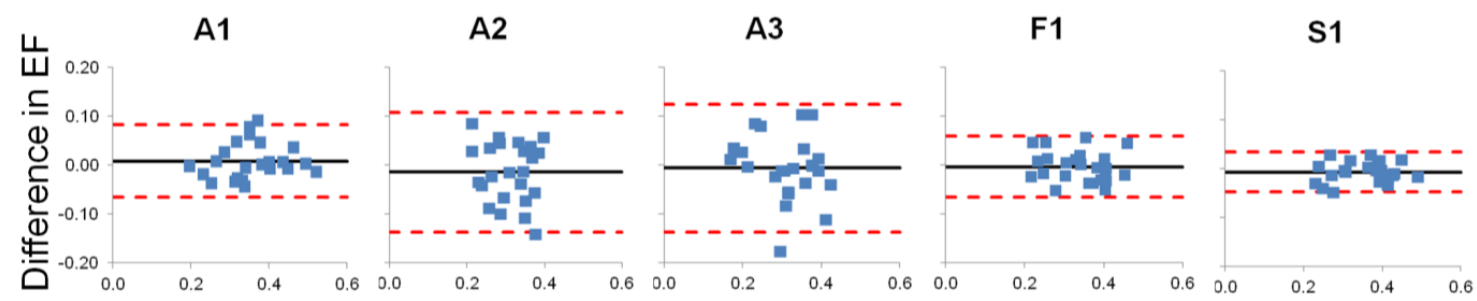

Interobserver variability:

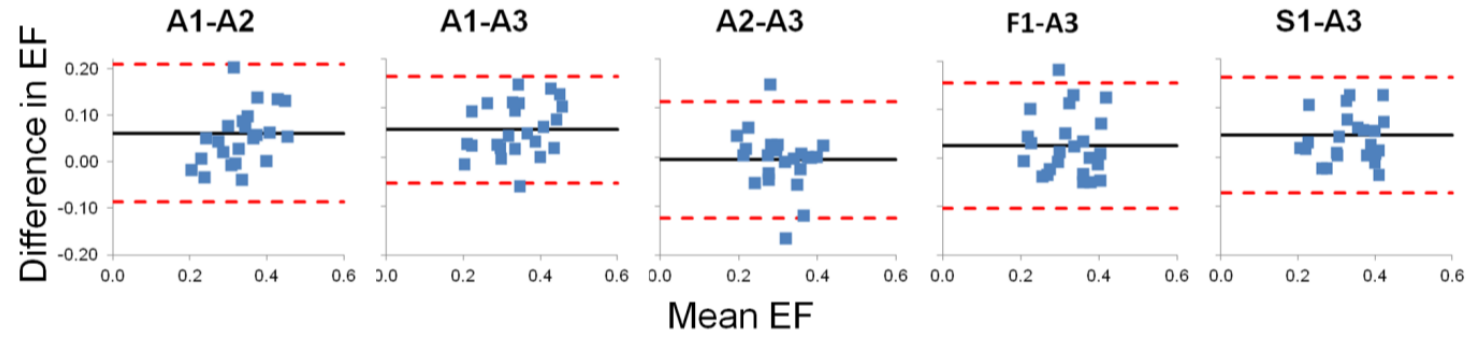

Figure 2.6 Bland-Altman analysis of EF intraobserver and interobserver variability

Bland-Altman analysis is used to evaluate intraobserver variability for each user $(\mathbf{A})$ and interobserver variability between attendings $(A 1, A 2, A 3)$ and between students $(F 1, S 1)$ and $A 3(B)$. 


\section{Sensitivity of Mechanical Measures to Force Generation in the Ischemic Region and Surrounding Myocardium}

\subsection{Introduction}

Over the last twenty years, methods for imaging heart function in patients have improved drastically. Most diagnoses were originally made by an experienced cardiologist who was trained to recognize different pathologies by eye in medical images. Recent advances in imaging technology allow in vivo quantitative measurements of myocardial mechanical function such as strain, strain rate, and wall motion, as described in Chapter 1. While this technology has increased the amount of information available from imaging, it can be difficult to determine the relative utility of different measures for detecting a particular pathology. Most research studies use one imaging modality to report one or two measures, and the sum of this literature can produce conflicting results. For example, for detecting coronary artery disease (CAD) from stress echocardiography, various studies have found that circumferential strain performs better than longitudinal and radial strain, ${ }^{93}$ circumferential and longitudinal strain perform better than radial strain, ${ }^{65}$ strain rate performs better than systolic strain ${ }^{66,94}$ but worse than post-systolic strain, ${ }^{66}$ and peak systolic strain rate from $2 \mathrm{D}$ speckle tracking performs better than tissue Doppler. ${ }^{67}$ While interesting comparisons, it can be difficult to translate results between studies as the data are collected from different imaging modalities, at different locations in the heart and with different sources or error.

It is also difficult to understand the factors that cause changes in these different measures from these limited data sets. Most experimental studies induce ischemia by 
occluding an artery, resulting in supply ischemia in a region of no blood flow and limited force generation surrounded by normally-contracting myocardium. In contrast, clinical studies usually induce ischemia in a region with limited hyperemic flow during a stress test, causing demand ischemia in the region that receives the same or slightly decreased blood flow as was delivered at baseline while surrounded by myocardium with increased contractility. Both blood flow to the ischemic region and contractility of the adjacent myocardium are different between demand and supply ischemia. While the relationship between regional mechanics and blood flow reduction has been thoroughly investigated,${ }^{29-31,33}$ the influence of the remote myocardium is not as well understood. In literature, mechanical tethering between the ischemic and non-ischemic myocardium has been studied in the "border zone" spanning the normally-perfused myocardium at the edge of the ischemic region. This region has been shown to have impaired circumferential and longitudinal strain ${ }^{41,95,96}$ and wall thickening ${ }^{97}$ during supply ischemia, causing mechanical abnormalities to appear larger than the region of abnormal perfusion. While the influence of tethering has been less studied in the context of demand ischemia, one study does report wall thickening abnormalities that are smaller than the corresponding perfusion abnormalities during stress testing ${ }^{98}$ suggesting that tethering and border zone mechanics in regions of demand ischemia may affect mechanical behavior more inside the perfusion defect than outside the defect as in supply ischemia.

Changes in remote contractility may impact the mechanical behavior in the ischemic region more in some directions than others. The remote myocardium impacts ischemic region behavior not only by applying force in the fiber direction, but also through shear in the cross-fiber and radial directions. Tethering in the fiber direction exerts normal forces across the ischemic region (Figure 3.1A). In the crossfiber-fiber 
plane, shear results in a resistance to bulging inside the ischemic region and resistance to contraction in the border zone in the crossfiber direction (Figure 3.1B). Crossfiberradial and fiber-radial shear also affects the radial direction through resistance to thinning inside the ischemic region and resistance to thickening outside the ischemic region. These effects have been observed in supply ischemia, where the border zone was observed to be larger in the cross-fiber direction than in the fiber direction. ${ }^{41}$ Finally, tethering to myocardium with inward wall motion restricts bulging, which we call "wall motion tethering" (Figure 3.1C). The influence of the border zone in this case is a function of both the contractility of the remote myocardium and the ischemic region size, as smaller ischemic regions are unable to bulge as much as large regions. Therefore, in this study we will investigate the influence of three different factors on the mechanics of an ischemic region: reduced active force generation in the ischemic region, the size of the ischemic region, and the contractility of the remote myocardium. A better understanding of the influence of these factors on different measures of regional mechanics could inform future directions in research and clinical use of mechanical measures for detecting CAD.

One way to understand the mechanisms underlying these clinical observations is through finite element (FE) modeling of the heart, in which a detailed model of the heart geometry, active and passive mechanical properties, and boundary conditions such as cavity pressure can be used to study the mechanical consequences of disease. FE modeling has been used in the past to determine the effect of infarct size and stiffness on global cardiac function, ${ }^{99}$ explain experimentally-observed differences in ischemic region border zone size,$^{41}$ test strategies for infarct reinforcement, ${ }^{83,100,101}$ and compare the sensitivity of various measures of dyssynchrony to functional changes caused by heart failure. ${ }^{102}$ A FE model of the heart at peak stress could help us understand both 
the individual effects of active force generation, remote contractility, and ischemic region size on the behavior of an ischemic region during stress testing, and the way these changes affect different measures of myocardial mechanical behavior. The goal of this study is to adapt a previously-developed FE model of the heart ${ }^{102}$ to model peak dobutamine stress based on experimental data, and then measure the sensitivity of various mechanical measures to reduced regional force generation, remote contractility, and ischemic region size during stress testing. This information will increase our understanding of the factors that affect regional mechanical behavior in demand ischemia, and allow better measurement selection for detecting CAD during dobutamine stress testing.

\subsection{Methods}

\subsubsection{FE Model}

To model cardiac function at baseline and peak stress, we modified a FE model originally published by Kerckhoffs et al. ${ }^{102}$ This model includes the 3D geometry and fiber architecture of the canine right and left ventricles (RV and LV, respectively) and a closed circulation model coupled to the ventricles. Ventricular geometry was represented by a mesh shown in Figure 3.2, with 40 elements representing the LV (4 circumferential, 3 longitudinal, 4 radial) and 8 representing the RV. Elements were described with cubic Hermite basis functions, and integration was performed at 27 Gauss points $(3 \times 3 \times 3)$ per element. The constitutive properties of passive myocardium in the model are expressed by a transversely isotropic exponential Fung-type strain energy function $W:^{103}$

$$
W=0.5 * C_{\text {pas }} *\left(e^{Q}-1\right)+0.5 * K * \ln (\mathrm{J})^{2}
$$




$$
Q=b_{f f} E_{f f}^{2}+b_{x x}\left(E_{c c}^{2}+E_{s S}^{2}+E_{c s}^{2}+E_{s c}^{2}\right)+b_{f x}\left(E_{f c}^{2}+E_{c f}^{2}+E_{f s}^{2}+E_{s f}^{2}\right)
$$

where $Q$ is a function of components of the Lagrangian strain tensor $\mathbf{E}$ : fiber strain $\left(E_{\mathrm{ff}}\right)$, cross-fiber strain $\left(E_{\mathrm{cc}}\right)$, radial strain $\left(E_{\mathrm{ss}}\right)$, and shear strains, and $J$ is the determinant of the deformation gradient tensor F. Normal passive canine myocardium was represented with the coefficients $C_{p a s}=0.88 \mathrm{kPa}, b_{\mathrm{ff}}=18.5, b_{\mathrm{xx}}=3.58$, and $b_{\mathrm{fx}}=1.63 .{ }^{103,104}$ The second term of Equation (1) was included with $K=100 \mathrm{kPa}$ to impose near incompressibility on the myocardium. ${ }^{105}$ Active contraction was simulated with a time- and length-dependent model of sarcomere mechanics ${ }^{106}$ with modified peak contractile velocity, ${ }^{102}$ in which the length of the contractile element $\left(L_{s c}\right)$ and a time-variant contractility parameter $(C)$ were state variables. Normalized length of the series elastic element $\left(L_{s N o r m}\right)$ was calculated by:

$$
L_{S N o r m}=\left(L_{S}-L_{S C}\right) / L_{S e r E l}
$$

where $L_{s}$ is sarcomere length and $L_{\text {SerEI }}$ is the length of the series elastic element during isometric contraction. Contractile element velocity $\left(\mathrm{dL}_{\mathrm{sd}} / \mathrm{d} t\right)$ and contractility $C$ are described by:

$$
\begin{aligned}
& \frac{d L_{s c}}{d t}=\left\{\begin{array}{cc}
\frac{L_{s N o r m}-1}{b_{\text {hill }} * L_{S N o r m}+1} v_{\text {max }} & L_{S N o r m} \leq 1 \\
\frac{L_{S N o r m}-1}{b_{\text {hill }} * L_{S N o r m}+1} v_{\text {max }} * e^{a_{\text {hill }}\left(L_{S N o r m}-1\right)} & L_{S N o r m}>1
\end{array}\right. \\
& \frac{d C}{d t}=\frac{1}{\tau_{r}} * C_{L} * f_{\text {rise }}+\frac{1}{\tau_{d}} \frac{C_{\text {rest }}-C}{1+e^{(T-t) / \tau_{d}}}
\end{aligned}
$$

where $t$ is time elapsed since electrical activation;

$$
C_{L}=\tanh \left(20\left(L_{s c}-L_{s 0}\right)^{2}\right)
$$

regulates contractility dependence on contractile element length;

$$
f_{\text {rise }}=0.02(8-x)^{2} x^{3} e^{-x}
$$

with $x=\min \left(8, \max \left(0, t / \tau_{\mathrm{r}}\right)\right.$ regulates rise of contractility and

$$
T=\tau_{s c}\left(0.29+0.3 L_{s c}\right)
$$


regulates twitch duration. Active fiber stress $\sigma_{t, a}$ is calculated by

$$
\sigma_{f, a}=\sigma_{a c t} * C *\left(L_{s c}-L_{s 0}\right) * L_{s N o r m}
$$

Active stress is also applied transverse to the myofibers using the methods of Lin and Yin, ${ }^{107}$ and was about $40 \%$ of active stress generated in the myofiber direction depending on local strain. Parameter values are listed in Table 3.1. The circulation was described by the CircAdapt model, ${ }^{108}$ a nonlinear lumped parameter systems model of the systemic and pulmonary circulation which includes nonlinear valve, vessel, and blood inertia behavior. Circulation model parameters are also listed in Table 3.1.

\section{Model Parameters at Stress}

Baseline function in the absence of dobutamine (-DOB) was modeled with active, passive, and circulation parameters described by Kerckhoffs et al, ${ }^{102}$ which are listed in Table 3.1. Table 3.2 describes the parameters that were changed to create a model of canine cardiac function during dobutamine infusion of $30 \mu \mathrm{g} / \mathrm{min} / \mathrm{kg}(+\mathrm{DOB})$, the source of the experimental data, and the methods used to derive the parameter value from the data. Parameters were altered so that the percent change between $-\mathrm{DOB}$ and +DOB was equal to the percent change observed in experimental data between baseline and peak dobutamine stress. For example, a change in the active stress scaling factor $\sigma_{\text {act }}$ changes the force generation of each fiber, so the percent change in $\sigma_{\text {act }}$ value, based on the previously-determined baseline value, was set to be equal to the percent change in ES pressure-volume relation slope observed experimentally. A similar approach was taken with $\mathrm{v}_{\max }$, because while the $\mathrm{v}_{\max }$ scaling factor included in the model is a different value than $v_{\max }$ measured from the derivative of the LV pressure signal, $\mathrm{dP} / \mathrm{dt}$, the change in these values is similar between baseline and stress. All altered parameter values are outlined in Table 3.3. Peripheral resistance and total blood volume were 
iterated by the circulation CircAdapt model to match a target mean aortic pressure and cardiac output. This iteration was used to determine peripheral resistance and total blood volume for $-\mathrm{DOB}$ and $+\mathrm{DOB}$, listed in Table 3.3, and then these values were used for all models of ischemia.

\section{Model Experiments}

Example Large and Small ischemic regions were selected from the canine experiments described in Chapter 4. Regional blood flow measurements by microspheres in 60 segments equally-spaced over the endocardial surface were converted to binary data to label each segment as ischemic or non-ischemic based on thresholds established in Chapter 4. A bicubic Hermite field function was fit to this binary data so that the location of the example ischemic regions on the LV surface were represented as a continuous function. The resulting ischemic regions, Large and Small, covered $32 \%$ and $17 \%$ of the LV surface, respectively, in the perfusion bed of the left anterior descending (LAD) artery on the apex and anterior wall of the LV. These ischemic regions are shown in Figure 3.2. Ischemia was modeled by varying $\sigma_{\text {act }}$ within the ischemic region. Mild supply ischemia was implemented in the -DOB model by decreasing $\sigma_{\text {act }}$ to $25 \mathrm{kPa}$ from $50 \mathrm{kPa}$, and severe ischemia by making $\sigma_{\text {act }}$ equal to zero. Demand ischemia caused by a critical stenosis was modeled by setting $\sigma_{\text {act }}$ in the ischemic region of the +DOB model equal to the baseline $\sigma_{\mathrm{act}}, 50 \mathrm{kPa}$ (referred to as $\sigma_{\mathrm{b}}$ ), representing a lack of increased blood flow. More severe demand ischemia was modeled by decreasing $\sigma_{\text {act }}$ to $25 \mathrm{kPa}$ (referred to as $\left.\sigma_{b} / 2\right)$

\section{Computational Details}

All models were implemented in Continuity ${ }^{\circledR} 6.4 \mathrm{~b}$ v7232 (www.continuity.ucsd.edu), a freely-available FE software package designed for cardiac modeling. Simulations were 
solved on a Dell Rocks Linux cluster with $2.4-\mathrm{GHz}$ Intel® Xeon processors and 2 GB of RAM. All simulations were solved with a timestep of $1 \mathrm{~ms}$, with 600 steps for baseline models and 340 steps for stress. Models were run until steady state, defined as the beat when the difference between LV and RV stroke volume was less than 1\%. Baseline ischemic models reached steady state after two to five beats, and all stress models reached steady state after 23 to 25 beats. Solution time was approximately 150 minutes per beat for baseline and 90 minutes per beat for stress on 12 processors running in parallel.

\subsubsection{Model Validation}

The $-\mathrm{DOB}$ and +DOB models were validated by comparing steady-state LV pressures, volumes, and anterior wall strains to those measured in the canine study of dobutamine stress described in Chapter 4. The +DOB models with large ischemic regions were validated by comparing to the STEN models of critical stenosis described in Chapter 4 . The -DOB model with a large ischemic region was compared to data reported previously by our lab, ${ }^{77}$ which was collected in dogs during 5 minute occlusion of the proximal LAD artery $(n=5)$. All pressures were measured with a Millar pressure catheter, volumes were measured from three-dimensional echocardiography (3DE) images, and strains were measured from a triangle of sonomicrometers in the midwall circumferential-longitudinal plane implanted in the LAD perfusion bed.

\subsubsection{Data Analysis}

Validation of regional mechanics and sensitivity analysis was completed with values measured at a Gauss point in the center of both ischemic regions, illustrated in Figure 
$3.2,40 \%$ through the wall thickness in the radial direction. This location, slightly more epicardial than the midwall, is similar to the location of the sonomicrometers used to measure strains in the ischemic region in Chapter 4. Strains were exported from the model in the circumferential $\left(\mathrm{E}_{11}\right)$, longitudinal $\left(\mathrm{E}_{22}\right)$, and radial $\left(\mathrm{E}_{33}\right)$ direction at a sampling rate of $1 \mathrm{~ms}$ through the cardiac cycle. All strains were computed in reference to the ED state, chosen just before the rise in LV pressure, and systolic strains were reported at ES, chosen just before the decrease in LV pressure. Principal strains in the circumferential-longitudinal plane were calculated from the eigenvalues of the twodimensional strain tensor in the circumferential-longitudinal plane, as detailed in Chapter 2. Wall thickening (WT) was measured by taking a short axis slice through the mesh at the endocardial longitudinal position of this measurement point, and measuring endocardial and epicardial location to calculate wall thickness. Strain rate was calculated by differentiating $E_{11}$ and $E_{22}$ with respect to time, and peak systolic strain rates $\left(\mathrm{SR}_{11}\right.$ and $\mathrm{SR}_{22}$ ) were defined as the peak negative value of the strain rate curve between the aortic valve opening and closing, as is commonly used in tissue Doppler image analysis $^{86}$. To measure wall motion, a dense mesh representing the endocardial surface was exported from the model at ED and ES and three-dimensional fractional shortening (3DFS) was calculated according to the methods detailed in Chapter 2. $\triangle$ 3DFS was calculated by subtracting the control 3DFS at each location on the LV surface. Strain or stress over the entire LV surface was displayed by measuring model strain at every Gauss point in the LV at this $40 \%$ depth, linearly interpolating over the LV surface, and displaying on a Hammer projection map.

The sensitivity of each measure to force generation in the ischemic region was evaluated by measuring absolute change of each measure between control and $\sigma_{\text {act }}=0$ during $-\mathrm{DOB}$ and between control and $\sigma_{\mathrm{act}}=\sigma_{\mathrm{b}} / 2$ in $+\mathrm{DOB}$ for the Large ischemic region. 
To compare these ranges among measures, the range was normalized by the standard deviation of each measure observed in the experiments described in Chapter 4. This approach highlights measures with a range that is large in comparison to the amount of normal observed variation. Measures from -DOB were normalized by standard deviations observed at rest with no occlusion, and measures from +DOB were normalized by standard deviations observed at peak dobutamine stress with no stenosis. Standard deviations are reported in Chapter 4 for $E_{11}, E_{22}$, area strain, $3 D F S, \mathrm{SR}_{11}$, and $S_{22}$. While $E_{33}$ was not measured in these experiments, we estimated $E_{33}$ from $E_{11}$ and $E_{22}$ by assuming incompressibility. The standard deviation of $E_{33}$ was 0.115 at baseline and 0.185 during peak stress, and values and standard deviations were similar to those previously observed in canines at rest. ${ }^{34}$ The standard deviation for WT was assumed to be equal to the standard deviation of $E_{33}$. Sensitivity to ischemic region size was evaluated by calculating the absolute change in each measure between the Small and Large regions in the $\sigma_{\mathrm{act}}=0$ models during $-\mathrm{DOB}$ and between control and $\sigma_{\mathrm{act}}=\sigma_{\mathrm{b}} / 2$ in + DOB.

\subsection{Results}

\subsubsection{Model Validation}

Most model pressures and volumes fell within one standard deviation of experimentallymeasured pressures and volumes (Figure 3.3). ED pressure (EDP) during +DOB, with and without ischemia, was higher in the model than in experiments. ES volume in -DOB, $+\mathrm{DOB}$, and $+\mathrm{DOB}$ with ischemia was larger than ES volume measured from experiments. This discrepancy in volume is not unexpected as the experimental volumes 
were measured from 3DE images, which are known to measure smaller than the true volume due to the appearance of trabeculae in the images. ${ }^{92}$

To validate regional mechanical behavior, strains and wall motion in the center of the anterior apical ischemic region were compared between experiments and the model (Figure 3.4A). Overall, we see that regional function in the model and data match quite well. In the -DOB model, all measures fall within one standard deviation of measured data except for $E_{22}$, which is smaller in the model than was measured in dogs. Data measured from occlusions of the proximal LAD were compared to models of Large ischemic regions with $\sigma_{\mathrm{act}}=0$ and $\sigma_{\mathrm{act}}=\sigma_{\mathrm{b}} / 2$, to provide context on how these theoretical models compare to a region of supply ischemia in vivo. We see that the patterns of change between no ischemia and ischemia are the same between the model and data, and that the experimental data falls within the span of the $\sigma_{\mathrm{act}}=0$ and $\sigma_{\mathrm{act}}=\sigma_{\mathrm{b}} / 2$ models; the Large $\sigma_{\mathrm{act}}=0$ model displays worse dysfunction than is observed in vivo, and the Large $\sigma_{\mathrm{act}}=\sigma_{\mathrm{b}} / 2$ model displays more contractile function than in vivo ischemia. The $\sigma_{\mathrm{act}}=0$ model provides a better match to the experiments because the same patterns of positive strains and negative 3DFS were observed, so the $\sigma_{\text {act }}=0$ model was chosen to represent acute supply ischemia for further sensitivity analysis.

For the $+\mathrm{DOB}$ model, shown in Figure $3.4 \mathrm{~B}, \mathrm{E}_{11}, \mathrm{E}_{22}$, and area strain match experimental data within one standard deviation while 3DFS is significantly smaller in the model compared to experimental data. In $+\mathrm{DOB}$ with a Large ischemic region, all measures from both the $\sigma_{\mathrm{act}}=\sigma_{\mathrm{b}}$ and $\sigma_{\mathrm{act}}=\sigma_{\mathrm{b}} / 2$ models fall within a standard deviation of the experimental data. The $\sigma_{\mathrm{act}}=\sigma_{\mathrm{b}} / 2$ model was chosen to represent demand ischemia for sensitivity analysis because regional strain in this model exhibited dysfunction similar to the more severe critical stenoses observed in our experiments. 


\subsubsection{Sensitivity Analysis}

Figure 3.5 shows each measure as a function of force generation in the ischemic region. By examining the patterns in this data, the sensitivity of each measure to ischemic force generation, remote contractility, and size can be evaluated. Every measure displays sensitivity to force generation during -DOB, as evident by the change in the red data points along the $\mathrm{x}$-axis. Measures were less sensitive overall to force generation during $+\mathrm{DOB}$, as the black data points changed less along the $\mathrm{x}$-axis. Area strain, 3DFS, and Peak $\mathrm{SR}_{11}$ displayed the highest sensitivity to force generation during +DOB. The sensitivity of each measure to ischemic region size can be evaluated by comparing the dotted and solid lines in each graph. Peak $\mathrm{SR}_{22}$ and 3DFS were sensitive to ischemic region size during both $-\mathrm{DOB}$ and $+\mathrm{DOB}$, while WT and $\mathrm{E}_{33}$ were also sensitive to size during +DOB. Finally, sensitivity to remote contractility can be evaluated by comparing the red and black data in each graph. Peak $\mathrm{SR}_{11}$ and Peak $\mathrm{SR}_{22}$ were very sensitive to remote contractility, 3DFS was somewhat sensitive, and the other measures were not.

To compare the effectiveness of different measures for detecting changes in ischemic region force generation or size, the absolute change in each measure from control was normalized by the standard deviation observed for that measure in the experiments detailed in Chapter 4. Overall, sensitivity to force generation was larger than sensitivity to region size (Figure 3.6). The left side of Figure 3.6A shows that during simulated supply ischemia $\left(-\mathrm{DOB} \sigma_{\mathrm{act}}=0\right)$, area strain changed the most relative to its experimental variation, followed by $E_{11}, 3 D F S$, and $E_{22}$. These measures were also the most sensitive to force generation during simulated demand ischemia (+DOB $\sigma_{a c t}=\sigma_{b} / 2$ ), shown on the right side of Figure 3.6A. Sensitivities in +DOB were lower than -DOB in every case but the relative sensitivities were mostly unchanged, i.e., area strain was the 
largest and Peak $\mathrm{SR}_{22}$ was smallest. While the magnitude of change in $\mathrm{SR}_{11}$ and $\mathrm{SR}_{22}$ was large with changing force generation, experimental variation was also large and resulted in lower sensitivity. The radial measures $E_{33}$ and WT were less sensitive to force generation than those in the circumferential-longitudinal plane. Sensitivity to ischemic region size, shown in Figure $3.6 \mathrm{~B}$, is of roughly the same magnitude during $-\mathrm{DOB}$ and $+\mathrm{DOB}$. The left side of Figure 3.6B shows that 3DFS was very sensitive to ischemic region size during $-\mathrm{DOB} \sigma_{\mathrm{act}}=0$, and $\mathrm{E}_{11}, \mathrm{WT}$, and $\mathrm{E}_{33}$ appeared independent of region size. Area strain, $E_{22}$, and $3 D F S$ were most sensitive to ischemic region size during $+\mathrm{DOB} \sigma_{\mathrm{act}}=\sigma_{\mathrm{b}} / 2$ (right), while Peak $\mathrm{SR}_{11}$ and Peak $\mathrm{SR}_{22}$ were less affected. Sensitivity to region size increased in area strain, $E_{11}, E_{22}, W T$, and $E_{33}$ between -DOB and $+\mathrm{DOB}$, and decreased in 3DFS, Peak $\mathrm{SR}_{11}$, and Peak $\mathrm{SR}_{22}$.

\subsubsection{Size of Mechanical Abnormalities}

Until recently, technical limitations prevented measurement of mechanics in more than a few locations in the myocardium. Now, 3D imaging modalities are increasingly available for clinical use, and fully 3D strain can be measured from 3DE or CMR at any location in the myocardium. How can this information be used to acquire more information on the size and location of an ischemic region? We addressed this question by measuring $E_{11}$, $E_{22}$, and $E_{33}$ over the entire LV of the models of supply ischemia $\left(\sigma_{a c t}=0\right)$ and $a$ hypothetical model of $+\mathrm{DOB}$ with ischemic $\sigma_{\mathrm{act}}=0$. This $+\mathrm{DOB} \sigma_{\mathrm{act}}=0$ model was used first to directly compare models that vary only in remote contractility. A threshold to separate normal function from abnormal function must be established to measure the size of a mechanical abnormality. Figure 3.7A shows the sizes of regions of abnormal strain when an abnormality is defined as bulging in the longitudinal or circumferential 
directions $\left(E_{11}\right.$ or $\left.E_{22}>0\right)$ or thinning in the radial direction $\left(E_{33}<0\right)$. The mechanical abnormalities appear larger in $-\mathrm{DOB}$ than in $+\mathrm{DOB}$ at matched ischemic region size, even though $\sigma_{\mathrm{act}}=0$ in both regions.

One possible reason for regions of abnormal function appearing smaller in +DOB compared to $-\mathrm{DOB}$ is that the threshold of abnormal function is different in the two cases. The ideal measure would detect a change from normal function. The model can be used to detect the locations where strain in the ischemic model has changed from the baseline $-\mathrm{DOB}$ or +DOB state. We measured $\Delta$ Strain, or the difference between strain in the control and ischemic models, over the entire LV. The size of the region where $\Delta$ Strain was greater than $5 \%$ is displayed in Figure 3.7B. Changes in circumferential or longitudinal strain can detect the size of an ischemic region very well. However, this change in radial strain still detected a wall motion abnormality smaller than the ischemic region, especially for $+\mathrm{DOB}$.

Comparing regions with $\sigma_{\mathrm{act}}=0$ in $-\mathrm{DOB}$ or $+\mathrm{DOB}$ models allows comparison of changes in remote contractility without the influence of force generation in the ischemic region, but the $+\mathrm{DOB} \sigma_{\mathrm{act}}=0$ model does not represent a real clinical state. Therefore, this $\Delta$ Strain analysis was repeated for the $\sigma_{a c t}=\sigma_{b} / 2$ models. The regions of abnormal $\Delta$ Strain in these models are smaller than the ischemic region, and regions of abnormal strain are smaller in -DOB models than in +DOB (Figure 3.7C). The region of abnormal $\Delta \mathrm{E}_{11}$ is close to ischemic region size in $+\mathrm{DOB}$, but $\Delta \mathrm{E}_{22}$ and $\Delta \mathrm{E}_{33}$ detect much smaller abnormalities.

Finally, this same analysis was completed for $\triangle 3 \mathrm{DFS}$ in the model. A $\triangle 3 \mathrm{DFS}<-$ $10 \%$ threshold was chosen to define the region of abnormal wall motion. The size of a $\triangle 3$ DFS abnormality was about equal to the size of the ischemic region during $\sigma_{\text {act }}=0$ in 
both $-\mathrm{DOB}$ and $+\mathrm{DOB}$ models (Figure 3.8A). When $\sigma_{\mathrm{act}}=\sigma_{\mathrm{b}} / 2$, such as in demand ischemia, the size of the $\triangle 3$ DFS abnormality was similar in the $-\mathrm{DOB}$ and +DOB models but smaller than the size of the ischemic region (Figure $3.8 \mathrm{~B}$ ). In this way, the size of a $\triangle$ BDFS abnormality is affected by ischemic force generation but not by remote contractility.

\subsection{Discussion}

A new FE model of cardiac mechanical function during peak dobutamine stress with and without a region of demand ischemia could predict experimentally-measured regional myocardial mechanical behavior with excellent agreement. This model was used to evaluate measures of regional cardiac mechanics for sensitivity to force generation in the ischemic region, contractility of remote myocardium, and ischemic region size. Area strain, $E_{11}, E_{22}$, and 3DFS were sensitive to force generation in the ischemic region for both $-\mathrm{DOB}$ and $+\mathrm{DOB}$, while $\mathrm{WT}, \mathrm{E}_{33}$, and Peak $\mathrm{SR}_{22}$ were less sensitive. Peak $\mathrm{SR}_{11}$ showed a large magnitude of change with force generation during both -DOB and $+\mathrm{DOB}$, but this change was small compared to its measurement variability. 3DFS in the center of the ischemic region was very sensitive to ischemic region size during -DOB, and $E_{11}, W T$, and $E_{33}$ appeared to be independent of region size. Area strain, $E_{22}$, and 3DFS were most sensitive to ischemic region size during +DOB, while Peak $\mathrm{SR}_{11}$ and Peak $\mathrm{SR}_{22}$ were least sensitive. Finally, strain rate was very sensitive to remote contractility, 3DFS was somewhat sensitive, and the other measures were not.

Further strain analysis over the entire surface of the LV showed that while the region of bulging (positive $E_{11}$ or $E_{22}$ ) or thinning (negative $E_{33}$ ) was always smaller than the ischemic region, the region of $\Delta$ Strain with a greater than $5 \%$ change from the 
control state is the same size as the ischemic region during $-\mathrm{DOB} \sigma_{\mathrm{act}}=0$. For $+\mathrm{DOB}$ $\sigma_{\text {act }}=0$ models, while the size of a region with abnormal $\Delta \mathrm{E}_{11}$ or $\Delta \mathrm{E}_{22}$ matches the size of the ischemic region well, the size of an $\Delta \mathrm{E}_{33}$ abnormality is smaller than the size of the ischemic region. During demand ischemia (+DOB $\left.\sigma_{a c t}=\sigma_{b} / 2\right)$, the $\Delta E_{11}$ abnormality matched the ischemic region best, and the $\Delta \mathrm{E}_{33}$ abnormality was much smaller than the ischemic region. The size of a $\triangle 3 \mathrm{DFS}$ abnormality matched the size of the ischemic region well for both $-\mathrm{DOB}$ and $+\mathrm{DOB} \sigma_{\mathrm{act}}=0$ models. In $\sigma_{\mathrm{act}}=\sigma_{\mathrm{b}} / 2$ models, the $\Delta 3 \mathrm{DFS}$ abnormality was smaller than the ischemic region but still changed with ischemic region size. These results will be helpful in interpreting in vivo studies of regional function during demand ischemia and in choosing the optimal measure for clinical applications.

\subsubsection{Comparison to Previous Studies}

The results of this study confirm some results from previous studies. For example, area strain as been proposed as a new measure because it combines both $E_{11}$ and $E_{22}$, and was shown to change more during supply ischemia than $E_{11}$ or $E_{22} \cdot{ }^{76}$ Here, we see that area strain is theoretically more sensitive than $E_{11}$ or $E_{22}$ in the case of demand ischemia as well. $E_{11}$ and $E_{22}$ have been reported to change more during demand ischemia than $\mathrm{E}_{33},{ }^{65,93}$ as is also observed in this model. The model provides more detail on this difference, as we see that the size of a radial strain abnormality is smaller than the size of the region with abnormal force generation. This radial abnormality is shown in more detail in Figure 3.9, where the geometry and wall thickness at ES in a circumferential line across the ischemic border zone is displayed for the Small ischemic region. While both $\sigma_{\text {act }}=0$ models display bulging compared to the control models, the endocardial border is closer to the epicardium for $-\mathrm{DOB}$ in the border zone (Figure 3.9A). This translates into 
impaired systolic wall thickness for more than $0.5 \mathrm{~cm}$ outside of the ischemic region in the $-\mathrm{DOB} \sigma_{\mathrm{act}}=0$ model of supply ischemia, while wall thickeness in the $+\mathrm{DOB} \sigma_{\mathrm{act}}=0$ model of demand ischemia only starts separating from control at the ischemic region border (Figure 3.9B). The border zone of impaired wall thickening clearly shifts with a change in remote contractility. This effect has been observed previously in canines, where the size of a perfusion defect during peak stress was always larger than the region of abnormal wall thickening. ${ }^{98}$ The model confirms these results, while also making it clear that these relationships are highly dependent on choice of cutoff for defining the region of abnormal function and that the same result may not have been seen if circumferential or longitudinal strain was used to measure the region of abnormal function.

These results also show that Peak $\mathrm{SR}_{11}$ may be better than Peak $\mathrm{SR}_{22}$ for detecting ischemia, demand or supply. $\mathrm{SR}_{22}$ has been studied more often because it is the easiest parameter to measure with tissue Doppler imaging. A number of different parameters derived from the $\mathrm{SR}_{22}$ signal have been proposed, ${ }^{66,109}$ and the value of the negative peak between aortic valve opening and closing has been used the most often. ${ }^{66,86-88,94}$ The sensitivity of Peak $\mathrm{SR}_{22}$ to demand ischemia has been shown to be limited in some studies in vivo, ${ }^{66,94}$ which is consistent with our model results. The measurement of strain rate with speckle tracking has become more common, making measurement of Peak $\mathrm{SR}_{11}$ feasible clinically, and this model indicates that using Peak $\mathrm{SR}_{11}$ may be better for detecting abnormal function. While this model has not been explicitly validated for measuring strain rate, as mentioned below, we will test these results in vivo in Chapter 4. 


\subsubsection{Clinical Implications}

When regional mechanics measures are evaluated clinically for detecting a critical stenosis, the results are influenced by not only by the effect of demand ischemia on mechanical behavior, but also by measurement noise and variability in perfusion abnormality size and severity between patients. The FE model is advantageous because there is no measurement noise. While a measure that is effective for detecting a region of demand ischemia in the model may end up appearing less effective clinically because of these complicating factors, a measure that is not effective in the model will likely not improve in vivo. In addition, and the size, location, and severity of the ischemic region in these models can be exactly specified. Different measures of regional mechanics likely have different sensitivities to force generation in the ischemic region, contractility of the remote myocardium, and ischemic region size. Understanding these differences allows better interpretation of an observed change in a measure, and may cause a user to select different measures for different goals.

While each measure has different behavior, we can see some patterns when using these criteria on the data in Figure 3.5. First, most measures clearly change more during ischemia in $-\mathrm{DOB}$ than in $+\mathrm{DOB}$. Area strain displayed the highest sensitivity to force generation, followed by $E_{22}, 3 D F S$, and $E_{11}$. Area strain, $E_{22}$, and $E_{11}$ were not sensitive to remote contractility or region size. These results suggest that a change in area strain, $E_{11}$, or $E_{22}$ during ischemia is due solely to force generation in the ischemic region and not region size or remote contractility. This confirms previous observations

that $E_{11}$ and $E_{22}$ are more effective for detecting demand ischemia than $E_{33},{ }^{65,93}$ and establishes the efficacy of area strain which has been tested at rest ${ }^{76}$ but not yet during stress. 3DFS was sensitive to both force generation and size, indicating that it could be 
an effective measure if information on both of these factors is desired. $E_{33}$ and WT, both measures in the radial direction, were sensitive to ischemic region size but not to force generation in +DOB models, and so may be effective for detecting large ischemic regions and not small ones. Finally, while Peak $\mathrm{SR}_{11}$ shows the largest observed magnitude of change, it is much different between $-\mathrm{DOB}$ and +DOB. This measure could be effective for detecting ischemia if different thresholds for abnormal values are set for different levels of remote contractility. Peak $\mathrm{SR}_{22}$ is not as sensitive to force generation as Peak $\mathrm{SR}_{11}$ but is affected by ischemic region size.

The significance of the magnitude of these sensitivities depends on the amount of variability observed in a measure in vivo. Figure 3.6 shows the range of change in each measure normalized by the standard deviation in observed in our canine study in Chapter 4. Most of the measures in the canine study were derived from sonomicrometry, which is very accurate for measuring strain, so this variability reflects more experimental physiologic variation than clinical measurement accuracy. Still, it provides context for comparing ranges between measures. In this comparison, area strain is the most sensitive to force generation during both $-\mathrm{DOB}$ and $+\mathrm{DOB}$, followed by $E_{22}, 3 \mathrm{DFS}$, and $E_{11}$. The sensitivity of Peak $S R_{11}$ is lower when normalized by measurement variability, although this estimation of measurement variability is likely high because the magnitude of Peak $\mathrm{SR}_{11}$ in Chapter 4 was larger than observed here. Peak $\mathrm{SR}_{11}$ was also less sensitive than the other measures to ischemic region size. Overall, these results indicate that strains in the circumferential-longitudinal plane and 3DFS appear more promising than radial measures for detecting demand ischemia during stress testing. These conclusions will be tested in Chapter 4 . 
While detecting demand ischemia during stress testing is important, the availability of quantitative measurement from fully 3D imaging can provide additional information on the size and location of the ischemic region. Theoretically, if a measure is only sensitive to local force generation and not region size or remote contractility, then the region with depressed function in this measure should correspond very closely with the ischemic region. A measure that is sensitive to ischemic region size and force generation may be advantageous if data can only be acquired in a few locations, but if fully $3 \mathrm{D}$ data is available perhaps only sensitivity to force generation is desirable. The results shown in Figure 3.7 show that while the region of bulging caused by a $\sigma_{\text {act }}=0$ ischemic region is larger in $-\mathrm{DOB}$ than in $+\mathrm{DOB}$, the size of a region with abnormal strain is the same in $-D O B$ and $+D O B$ for $E_{11}$ and $E_{22}$ but not $E_{33}$. Therefore, not only is $E_{33}$ less sensitive to reduced force generation during $+D O B$, the size of an $E_{33}$ abnormality is smaller than the size of the ischemic region. The fact that $E_{33}$ changes with ischemic region size and is not decreased in some locations with reduced force generation suggests that it is affected by the behavior of the surrounding myocardium. These observations suggest that $\mathrm{E}_{33}$ or WT may not be the best measures for measuring the size of demand ischemia.

Our group designed 3DFS as a 3D measure for the purpose of measuring the size of a wall motion abnormality, ${ }^{78}$ and here we see that it measures the size of an ischemic region well. Unlike strain, $\triangle 3$ DFS abnormality size does not change between -DOB and +DOB models, and therefore is not sensitive to remote contractility. This suggests that the normalization of 3DFS is effective in controlling for the effects of remote contractility, whereas even when control regional strain was subtracted to calculate $\Delta$ Strain, the regions of abnormal strain during $\sigma_{a c t}=\sigma_{b} / 2$ were smaller in $-D O B$ 
than in + DOB. Even though it is a measure in the radial direction, $\triangle$ 3DFS does not appear to be susceptible to the border zone effects that were apparent for $E_{33}$. While $\triangle$ 3DFS abnormality size was smaller in $\sigma_{a c t}=\sigma_{b} / 2$ regions, this is partly caused by threshold selection; a less stringent threshold would show abnormalities equal to the ischemic region size in $\sigma_{a c t}=\sigma_{b} / 2$ regions and larger than the ischemic region size in $\sigma_{\mathrm{act}}=0$. However, if ischemic regions with some active force generation require a less stringent threshold for the $\triangle 3$ DFS abnormality size to match ischemic region size, that threshold may identify more false positive wall motion abnormalities in vivo. A more stringent threshold may be more specific measure a smaller $\triangle 3 D F S$ abnormality size. We observed this effect in Chapter 4, where the $\triangle 3$ DFS threshold with the highest sensitivity and specificity measured abnormalities that were smaller than the ischemic region. Overall, the measures that were most sensitive to force generation in the center of the ischemic region, 3DFS and strain in the $E_{11}-E_{22}$ plane, were also most effective for measuring ischemic region size and show promise for measuring the size of an ischemic region clinically.

\subsubsection{Model Validation}

This FE model was able to replicate global pressures and volumes and regional strains remarkably well with a small number of parameter adjustments based on experimental data. Nevertheless, this model lacks some details which should be considered when interpreting the results of this study. This FE model of the heart during dobutamine stress is a novel adaptation of previous modeling efforts. ${ }^{110,111}$ The original model has been continuously improved and adapted to include more anatomic detail ${ }^{36}$ for accurate measurement of regional strains, ${ }^{40}$ and more realistic circulatory boundary conditions. ${ }^{37}$ 
Systolic strains measuring the change between ED and ES in the circumferentiallongitudinal plane have been compared to experimental data in both this study and previously. ${ }^{41}$ The circulation model has been shown to generate accurate global behavior during the cardiac cycle, ${ }^{37}$ but strains throughout the cardiac cycle with this circulation model, not just for the ED and ES time points, have not been evaluated before now. Measured strain rates are larger than those observed here, in Chapter 4 and elsewhere, and so verification of strain rate results will require verification in vivo, which will be completed in Chapter 4. Fiber angles are incorporated into the model but fiber sheets are not included. The mechanisms governing sheet mechanics are still not well-understood, but the transverse sheet shear has been measured to contribute significantly to $W T$ and $E_{33}$ in canines. ${ }^{112} A$ comparison of model results for WT and $E_{33}$ to previous studies in literature reveals that this model underestimates these measures. $E_{33}$ at baseline and stress in this model were both near 0.1 , whereas in previous studies $E_{33}$ measured with biplane imaging of implanted beads or sonomicrometry has been reported to be equal to 0.15 to 0.2 at baseline $e^{34,112,113}$ and 0.2 to 0.25 at stress. ${ }^{64} \mathrm{WT}$ measured by implanted markers in dogs has been measured to increase from 0.23 at baseline to 0.28 during dobutamine stress. ${ }^{81}$ From this data we can conclude that $E_{33}$ and WT in the model are lower than measured experimentally, and this is likely because of the lack of sheet structure and shear in the model. Nevertheless, the model still shows depressed WT and $E_{33}$ with demand ischemia and supply ischemia, and replicates previously-observed results in which a WT abnormality is smaller than a perfusion abnormality at peak stress. ${ }^{98}$ These model results for WT and $E_{33}$ indicate interesting differences between mechanics in the circumferential-longitudinal plane and the radial directions, but require further investivation in vivo. 
In our validation data, one in vivo pattern which was not replicated by the model was the increase in 3DFS from baseline to peak stress. In general, 3DFS magnitude was less in the model than in experiments. The likely cause of this discrepancy is the influence of trabeculae on the appearance of the endocardial border in 3DE. The reason volumes measured from $3 \mathrm{DE}$ are less than those measured by CMR is because trabeculae appear the same as endocardium in 3DE. ${ }^{92}$ Our lab has previously observed larger values of 3DFS measured in CMR compared with 3DE in the same patients, ${ }^{80}$ likely due to the same effect. The LV cavity was nearly obliterated in the canine peak stress images in the Chapter 4 study, and while the segmentation excluded papillary muscles and visible trabeculae, it is possible that the dogs had a large amount of trabeculae which made volumes appear smaller and 3DFS appear larger, especially during + DOB. 3DFS during demand ischemia was more similar in the model and data, and because sensitivity measures were calculated as a change from control, the sensitivity of 3DFS was likely underestimated. Even though this is a limitation of the model, the fact that 3DFS was still fairly sensitive to ischemia in this study indicates that this measure is promising for detecting demand ischemia in vivo.

\subsubsection{Conclusion}

By using a theoretical FE model of cardiac mechanics, prospective measures of regional mechanics were evaluated for sensitivity to demand ischemia. Area strain was the most sensitive to reduced force generation, and strains in the circumferential-longitudinal plane, wall motion measured by 3DFS, and circumferential strain rate showed more sensitivity than radial measures of strain or wall thickening. Regions of abnormal radial strain were shown to be smaller than the ischemic region at peak stress, while 
circumferential and longitudinal strain and 3DFS were abnormal in the entire ischemic region. These results clarify differences in measures observed in previous studies, and will be tested in vivo in later chapters. 
Table 3.1 Model parameters

\begin{tabular}{|c|c|}
\hline $\begin{array}{l}\text { Parameter Description and Unit } \\
\text { Active Contraction }\end{array}$ & Value \\
\hline $\begin{array}{l}a_{\text {Hill }}: \text { curvature of Hill relation during stretching }[-] \\
\mathrm{C}_{\text {rest }}: \text { Diastolic contractility }[-] \\
\mathrm{b}_{\mathrm{H} \text { Hill }}: \text { curvature of Hill relation during shortening }[-] \\
\mathrm{L}_{\mathrm{s} 0}: \text { contractile element length at zero active stress }[\mu \mathrm{m}] \\
\mathrm{L}_{\mathrm{SerEl}}: \text { length of series elastic element during isometric contraction }[\mu \mathrm{m}] \\
\mathrm{V}_{\text {max }}: \text { unloaded sarcomere shortening velocity }[\mu \mathrm{m} / \mathrm{sec}] \\
\tau_{\mathrm{d}}: \text { relaxation time scaling factor }[\mathrm{ms}] \\
\tau_{\mathrm{r}}: \text { contraction rise time scaling factor }[\mathrm{ms}] \\
\tau_{\mathrm{sc}}: \text { twitch duration scaling factor }[\mathrm{ms}] \\
\sigma_{\text {act }}: \text { active stress scaling factor }[\mathrm{kPa}]\end{array}$ & $\begin{array}{l}1.5 \\
0.0 \\
1.5 \\
1.51 \\
0.04 \\
5.0 \\
33.8 \\
28.1 \\
292.5 \\
50\end{array}$ \\
\hline
\end{tabular}

\section{Circulation}

Left and right atrium

Atrial-ventricular activation delay time [ms] $\quad 120$

Atrial inflow lumen area $\left[\mathrm{cm}^{2}\right] \quad 3.6$

Atrial inflow lumen length [cm] 1.26

Atrial reference midwall area $\left[\mathrm{cm}^{2}\right] \quad 46.5$

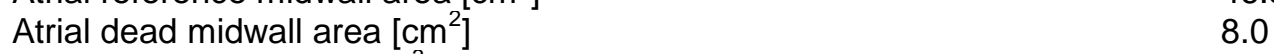

$\begin{array}{ll}\text { Left atrial wall volume }\left[\mathrm{cm}^{3}\right] & 7.45\end{array}$

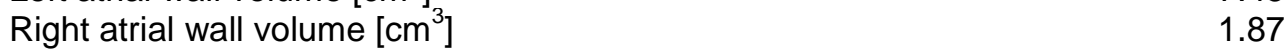

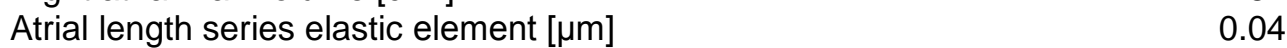

Atrial contraction rise time factor [ms] 45

Atrial relaxation time factor [ms] 30

Atrial twitch duration [ms] 200

Atrial sarcomere length when active stress is zero $[\mu \mathrm{m}] \quad 1.51$

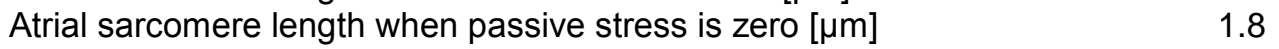

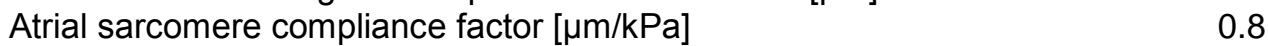

Atrial passive fiber stress scaling factor [kPa] $\quad 23.7$

Atrial resting contractility [-] $\quad 0.02$

Atrial sarcomere reference length $[\mu \mathrm{m}]$

Atrial active fiber stress scaling factor [kPa] $\quad 84.0$

Atrial unloaded sarcomere shortening velocity $[\mu \mathrm{m} / \mathrm{sec}] \quad 10$

Ventricular valves

Mitral valve lumen area when open $\left[\mathrm{cm}^{2}\right]_{2}$

Mitral valve lumen area when closed $\left[\mathrm{cm}^{2}\right] \quad 5 e-6$

Mitral valve orifice length [cm] $\quad 0.79$

Aortic valve lumen area when open $\left[\mathrm{cm}^{2}\right] \quad 2.5$

Aortic valve lumen area when closed $\left[\mathrm{cm}^{2}\right] \quad 2 \mathrm{e}-6$

Aortic valve orifice length [cm] $\quad 0.72$

Tricuspid valve lumen area when open $\left[\mathrm{cm}^{2}\right] \quad 4.76$

Tricuspid valve lumen area when closed $\left[\mathrm{cm}^{2}\right] \quad 5 e-6$

$\begin{array}{ll}\text { Tricuspid valve orifice length [cm] } & 0.79\end{array}$

Pulmonary valve lumen area when open $\left[\mathrm{cm}^{2}\right]$

Pulmonary valve lumen area when closed $\left[\mathrm{cm}^{2}\right] \quad 2 e-6$

$\begin{array}{ll}\text { Pulmonary valve orifice length }[\mathrm{cm}] & 0.72\end{array}$

\section{Aorta}

Wall cross-sectional area $\left[\mathrm{cm}^{2}\right] \quad 0.99$

Unloaded lumen area $\left[\mathrm{cm}^{2}\right] \quad 2.5$

Length of aorta [cm] 25.2 


\begin{tabular}{|c|c|}
\hline Reference pressure $[\mathrm{kPa}]$ & 12.3 \\
\hline Wall stress power scaling factor [-] & 1.7 \\
\hline \multicolumn{2}{|l|}{ Vena Cava } \\
\hline Wall cross-sectional area $\left[\mathrm{cm}^{2}\right]$ & 0.13 \\
\hline Unloaded lumen area $\left[\mathrm{cm}^{2}\right]$ & 2.5 \\
\hline Length of pulmonary artery $[\mathrm{cm}]$ & 25.2 \\
\hline Reference pressure $[\mathrm{kPa}]$ & 0.34 \\
\hline Wall stress power scaling factor & 2.3 \\
\hline \multicolumn{2}{|l|}{ Pulmonary artery } \\
\hline Wall cross-sectional area $\left[\mathrm{cm}^{2}\right]$ & 0.23 \\
\hline Unloaded lumen area $\left[\mathrm{cm}^{2}\right]$ & 2.02 \\
\hline Length of pulmonary artery [cm] & 12.6 \\
\hline Reference pressure $[\mathrm{kPa}]$ & 2.4 \\
\hline Wall stress power scaling factor [-] & 1.7 \\
\hline \multicolumn{2}{|l|}{ Pulmonary vein } \\
\hline Wall cross-sectional area $\left[\mathrm{cm}^{2}\right]$ & 0.2 \\
\hline Unloaded lumen area $\left[\mathrm{cm}^{2}\right]$ & 2.0 \\
\hline Length of pulmonary vein [cm] & 12.6 \\
\hline Reference pressure $[\mathrm{kPa}]$ & 0.94 \\
\hline Wall stress power scaling factor [-] & 2.3 \\
\hline Pulmonary drop pressure [kPa] & 1.5 \\
\hline
\end{tabular}




\section{Table 3.2 +DOB parameter sources}

\section{Parameter Component Data Source Analysis Methods}

\begin{tabular}{|c|c|c|c|}
\hline HR (bpm) & $\begin{array}{l}\text { Active, } \\
\text { circulation }\end{array}$ & Chapter 4 & Inverse of cycle time \\
\hline$\tau_{\mathrm{sc}}(\mathrm{ms})$ & Active & Chapter 4 & Time to ES as a fraction of cycle time \\
\hline $\mathrm{v}_{\max }(\mu \mathrm{m} / \mathrm{sec})$ & Active & Chapter 4 & $\begin{array}{l}\text { Calculated from } \mathrm{dP} / \mathrm{dt} \text { during isovolumic } \\
\text { contraction }^{114}\end{array}$ \\
\hline$\sigma_{\text {act }}(\mathrm{kPa})$ & Active & Literature $^{115}$ & $\begin{array}{l}\text { Slope of ES pressure-volume } \\
\text { relationship }\end{array}$ \\
\hline $\mathrm{CO}(\mathrm{L} / \mathrm{min})$ & Circulation & Chapter 4 & Stroke volume ${ }^{*} \mathrm{HR}$ \\
\hline MAP $(\mathrm{kPa})$ & Circulation & Chapter 4 & $\begin{array}{l}\text { Mean of peak isovolumic contraction LV } \\
\text { pressure and peak systolic LV pressure }\end{array}$ \\
\hline $\mathrm{t}_{\mathrm{act}, \mathrm{a}}(\mathrm{ms})$ & Circulation & Chapter 4 & Scale with change in HR \\
\hline$\tau_{\mathrm{a}}(\mathrm{ms})$ & Circulation & Chapter 4 & Scale with change in HR \\
\hline
\end{tabular}

$\mathrm{HR}=$ heart rate, $\tau_{\mathrm{sc}}=$ twitch duration scaling factor, $\mathrm{v}_{\max }=$ unloaded sarcomere shortening velocity, $\mathrm{dP} / \mathrm{dt}=$ derivative of $\mathrm{LV}$ pressure with respect to time, $\sigma_{\mathrm{act}}=$ peak active stress generation, $\mathrm{CO}=$ cardiac output, $\mathrm{MAP}=$ mean aortic pressure, $\mathrm{t}_{\mathrm{act}, \mathrm{a}}=$ atrial activation time relative to ventricular activation, $\tau_{\mathrm{a}}=$ atrial twitch duration. 
Table 3.3 +DOB parameters, model vs. data

\begin{tabular}{|c|c|c|c|c|c|c|c|}
\hline \multirow{2}{*}{$\begin{array}{l}\text { Data } \\
\text { Parameter }\end{array}$} & \multirow[b]{2}{*}{$-D O B$} & \multirow[b]{2}{*}{$+D O B$} & \multicolumn{2}{|l|}{ Model } & \multirow[b]{2}{*}{$+D O B$} & \multicolumn{2}{|c|}{$\%$ Increase } \\
\hline & & & Parameter & $-D O B$ & & Data & Model \\
\hline $\mathrm{HR}$ (bpm) & 97 & 174 & HR (bpm) & 100 & 176 & 79 & 76 \\
\hline$\tau_{\mathrm{sc}}($ fract cycle $)$ & 0.4 & 0.3 & $\tau_{\mathrm{sc}}(\mathrm{ms})$ & 292.5 & 257.4 & -13 & -12 \\
\hline $\mathrm{v}_{\max }(\mathrm{mm} / \mathrm{s})$ & 2.39 & 8.71 & $\mathrm{v}_{\max }(\mu \mathrm{m} / \mathrm{sec})$ & 0.005 & 0.0133 & 264 & 166 \\
\hline \multicolumn{8}{|l|}{ ESPVR slope } \\
\hline$(\mathrm{mmHg} / \mathrm{ml})$ & 6.7 & 12.5 & $\sigma_{\mathrm{act}}(\mathrm{kPa})$ & 50.0 & 96.7 & 87 & 93 \\
\hline $\mathrm{CO}(\mathrm{L} / \mathrm{min})$ & 1.9 & 4.4 & $\mathrm{CO}(\mathrm{L} / \mathrm{min})$ & 1.8 & 4.0 & 137 & 127 \\
\hline \multirow[t]{5}{*}{ MAP (kPa) } & 10.3 & 14.0 & MAP (kPa) & 9.0 & 12.3 & 36 & 36 \\
\hline & & & $\mathrm{t}_{\mathrm{act}, \mathrm{a}}(\mathrm{ms})$ & -120 & -68 & & -43 \\
\hline & & & $\tau_{\mathrm{a}}(\mathrm{ms})$ & 200 & 113 & & -44 \\
\hline & & & $\mathrm{R}_{\mathrm{p}}(\mathrm{kPa} \cdot \mathrm{s} / \mathrm{L})$ & 295 & 170 & & -42 \\
\hline & & & $\mathrm{V}_{\text {blood }}(\mathrm{ml})$ & 262 & 318 & & -21 \\
\hline
\end{tabular}

$\mathrm{HR}=$ heart rate, $\tau_{\mathrm{sc}}=$ twitch duration scaling factor, $\mathrm{v}_{\max }=$ unloaded sarcomere shortening velocity, $\mathrm{ESPVR}=\mathrm{ES}$ pressure-volume relationship, $\sigma_{\mathrm{act}}=$ peak active stress generation, $\mathrm{CO}=$ cardiac output, MAP $=$ mean aortic pressure, $t_{\mathrm{act}, \mathrm{a}}=$ atrial activation time relative to ventricular activation, $\tau_{a}=$ atrial twitch duration, $R_{p}=$ peripheral resistance of veins, $V_{\text {blood }}$ $=$ total blood volume. 

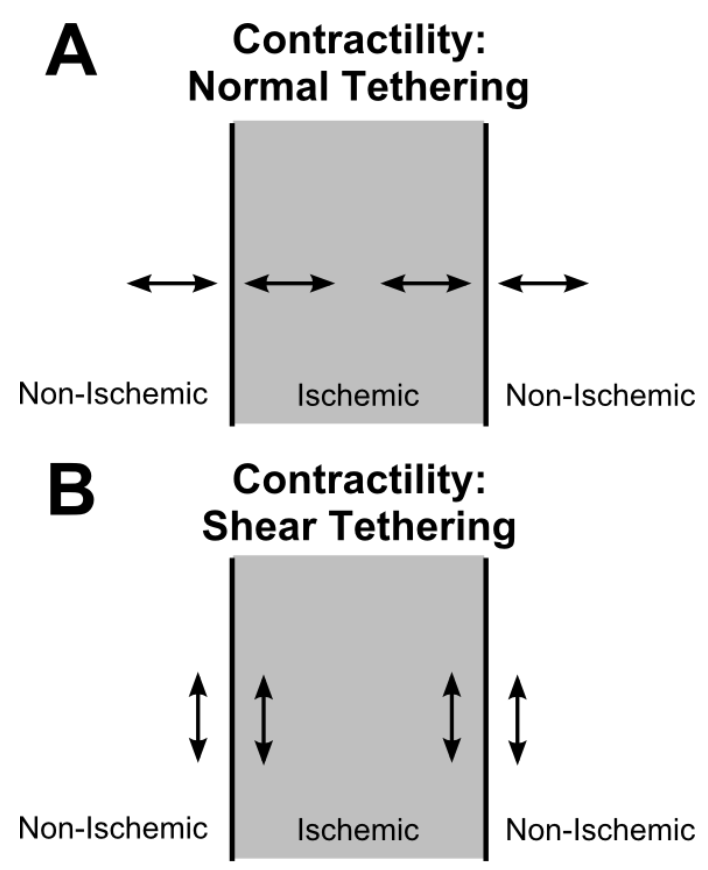

\section{Contractility and Size: \\ Wall Motion Tethering}

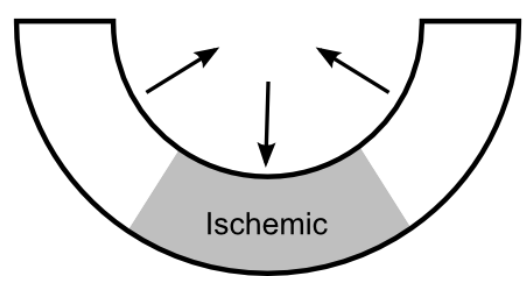

Figure 3.1 Tethering to adjacent myocardium

Diagram of the types of tethering to adjacent myocardium that affect regional mechanics in the ischemic region. Tethering normal to the ischemic region border, mostly in the fiber direction, increases fiber stress in the ischemic region when remote contractility is increased (A). Shear tethering parallel to the ischemic region border prevents sudden changes in mechanical function even if perfusion suddenly changes and may increase with remote contractility (B). Wall motion tethering inhibits outward wall motion in an ischemic region attached to inward-moving normal myocardium, to an extent that depends on both contractility and the size of an ischemic region (C). 

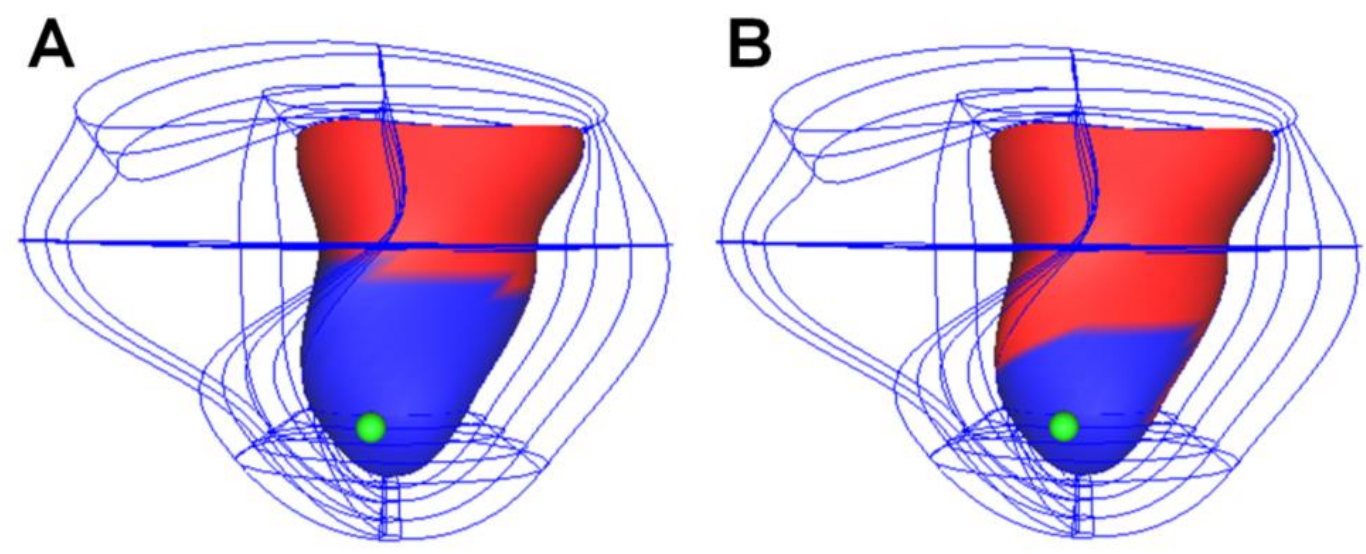

Figure 3.2 FE mesh geometry

Anterior view of the FE mesh used to model ventricular mechanics. Element boundaries are shown in blue. The endocardial surface is colored red for normal myocardium, and the small $(\mathbf{A})$ and large $(\mathbf{B})$ ischemic regions are colored in blue. The green dot marks the location of mechanical measurements used for sensitivity analysis. 


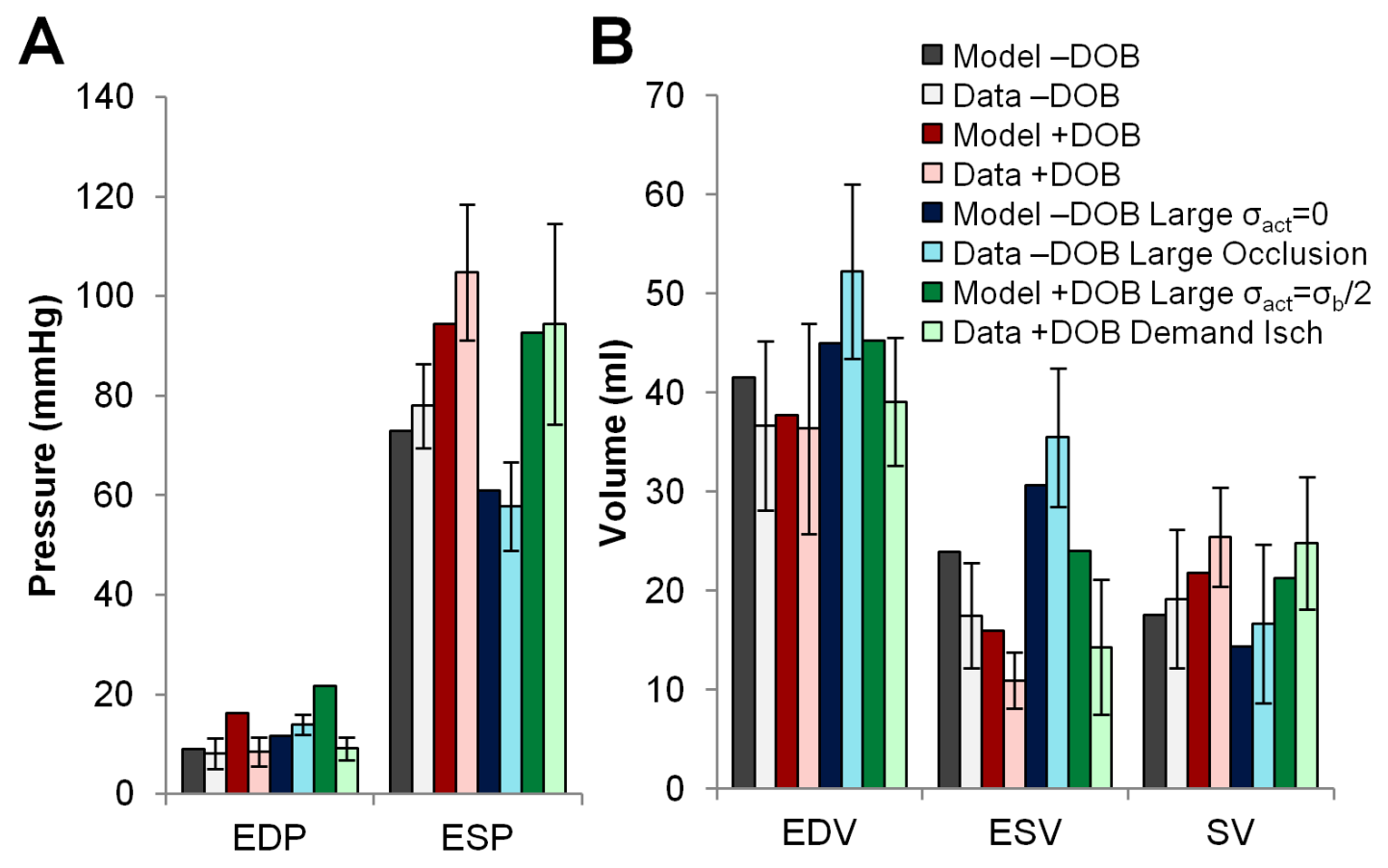

Figure 3.3 Validation of model global hemodynamic results

Model LV pressures (A) and LV volumes (B) match well with canine experimental data at baseline and peak stress. EDP $=E D$ pressure, ESP $=E S$ pressure, $E D V=E D$ volume, $\mathrm{ESV}=\mathrm{ES}$ volume, $\mathrm{SV}=$ stroke volume, $-\mathrm{DOB}=$ baseline without dobutamine, $+\mathrm{DOB}=$ peak dobutamine stress, $\sigma_{\mathrm{b}}=$ peak active stress during baseline conditions Error bars represent \pm 1 standard deviation. 


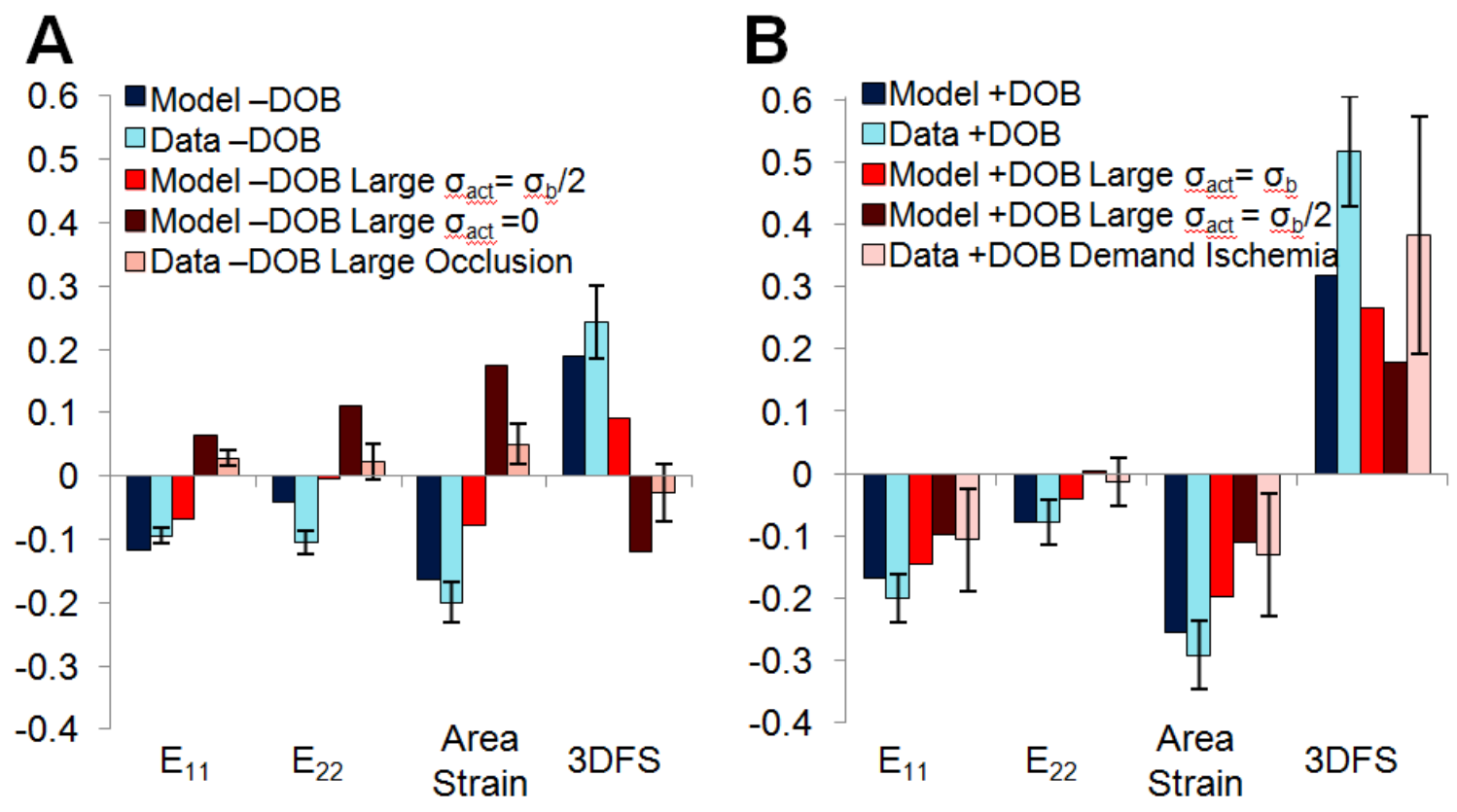

Figure 3.4 Validation of model regional mechanics

Model $E_{11}, E_{22}$, area strain, and 3DFS match experiments within one standard deviation at baseline $(\mathbf{A})$ and stress $(\mathbf{B})$, with the exception of depressed $E_{22}$ at baseline and depressed 3DFS at stress. Experimental mechanical dysfunction due to a large acute occlusion at baseline was less severe than the model with no force generation in the ischemic region $\left(\sigma_{\text {act }}=0\right)$ but more severe than the model in which ischemic region peak force generation $\sigma_{\mathrm{act}}=\sigma_{\mathrm{b}} / 2$ model, where $\sigma_{\mathrm{b}}$ is the peak force generation in the baseline model $(\mathbf{A})$. At stress, both the critical stenosis model $\left(\sigma_{a c t}=\sigma_{b}\right)$ and the severe stenosis model $\left(\sigma_{a c t}=\sigma_{b} / 2\right)$ fell within a standard deviation of experimental data measured during demand ischemia (B). 

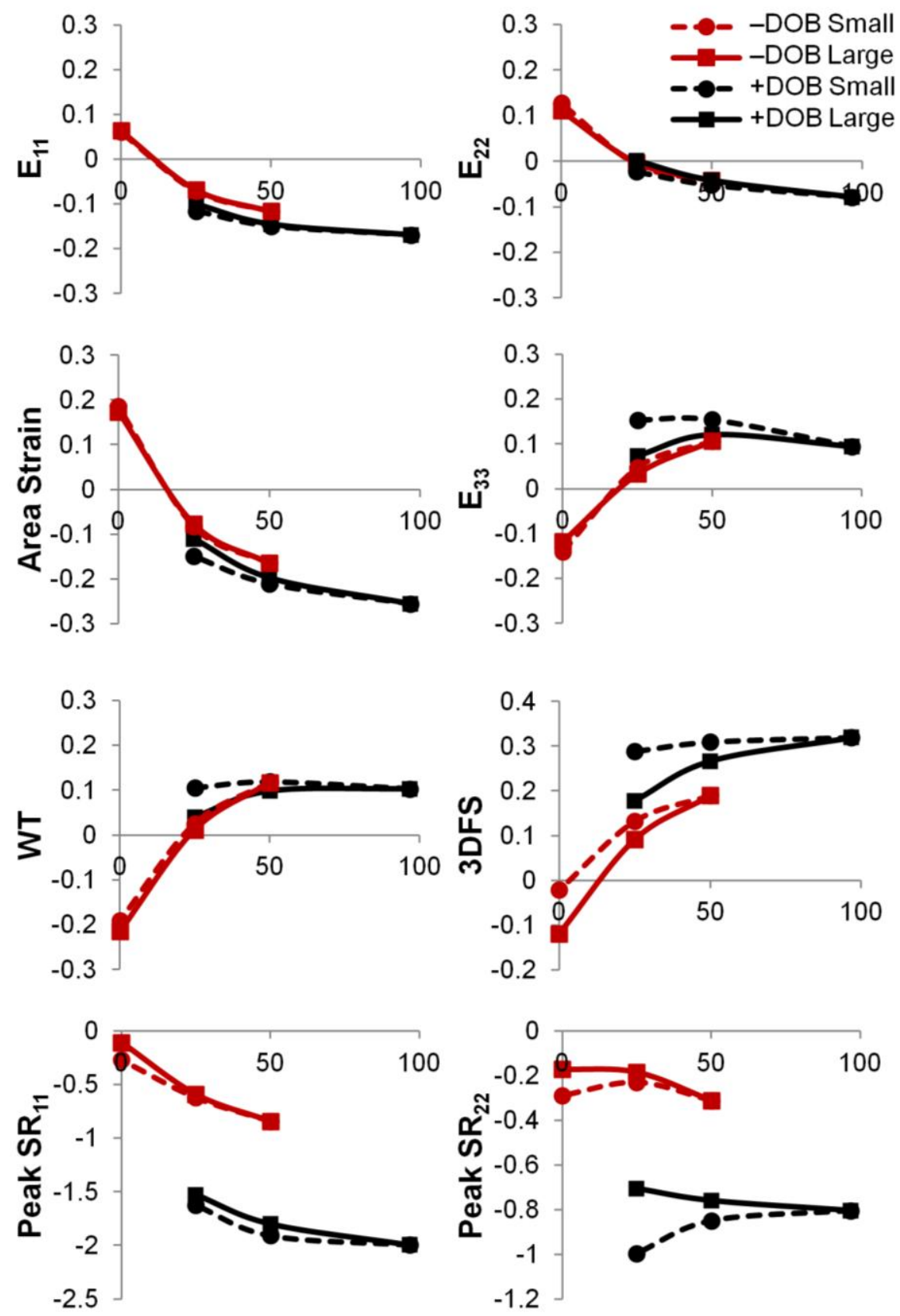

Ischemic Region Peak Force Generation (kPa)

Figure 3.5 Mechanical measures vs. ischemic force generation

Mechanical measures in the center of the ischemic region change with the peak force generation in the ischemic region, the behavior of the adjacent myocardium (-DOB or $+\mathrm{DOB})$, and ischemic region size. 


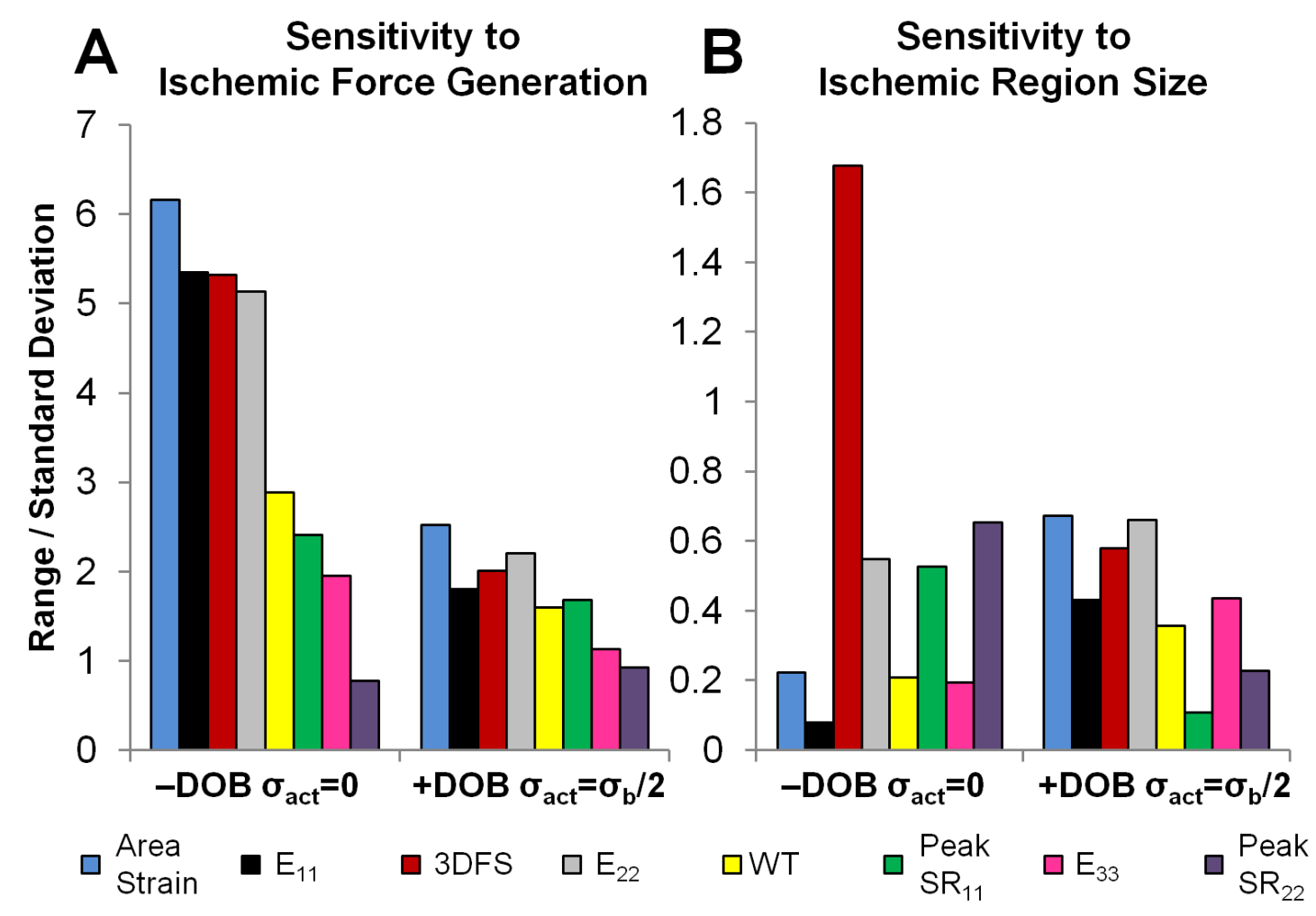

Figure 3.6 Measure sensitivity to ischemic force generation and size

Sensitivity of each mechanical measure to peak force generation in the ischemic region and ischemic region size was evaluated by displaying the absolute change from control measured in the center of a Large ischemic region. Control was defined as Baseline or Stress with no ischemia. Peak strain rate was very sensitive to force generation, and strain in the circumferential-longitudinal plan was less sensitive (A). Strain rate, especially in the longitudinal direction, is also sensitive to ischemic region size, as is 3DFS (B). 
$\begin{array}{ccc}\text { A Ischemic } \sigma_{\text {act }}=0 & \text { B } \begin{array}{c}\text { Ischemic } \sigma_{\text {act }}=0 \\ \text { Strain }>0 \% \text { Region }\end{array} & \begin{array}{r}\text { Ischemic } \sigma_{\text {act }}=\sigma_{\mathrm{b}} / 2 \\ \Delta \text { Strain }>5 \% \text { Region }\end{array} \\ \Delta \text { Strain }>5 \% \text { Region }\end{array}$

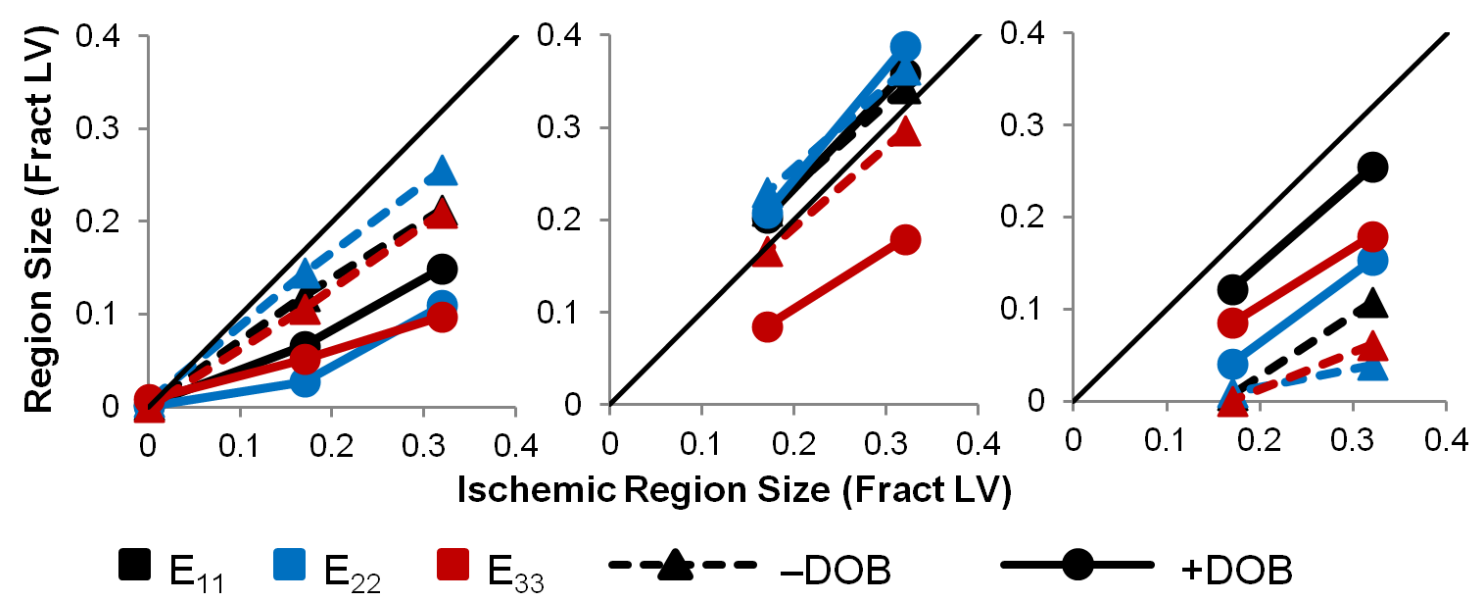

Figure 3.7 Size of strain abnormalies

The fraction of the LV surface with stretching $\left(E_{11}\right.$ or $\left.E_{22}\right)$ or thinning $\left(E_{33}\right)$ was measured in baseline and stress models with ischemic force generation $\sigma_{\mathrm{act}}=0(\mathbf{A})$. The size of a mechanical abnormality was always larger in baseline (supply ischemia) compared with stress (demand ischemia). When calculating the change in strain from control ( $\Delta$ Strain), the size of the region in which change in which $\Delta$ Strain is greater than $5 \%$ is very similar when ischemic $\sigma_{\text {act }}=0$ in all cases except $E_{33}$ during stress (B). During $+\mathrm{DOB}$ when ischemic $\sigma_{a c t}=\sigma_{b} / 2$, similar to demand ischemia, the size of $E_{11} \Delta$ Strain abnormality best captures the size of the ischemic region, followed by $E_{22}$ then $E_{33}$ while abnormality sizes were smaller in all measures during -DOB (C). 


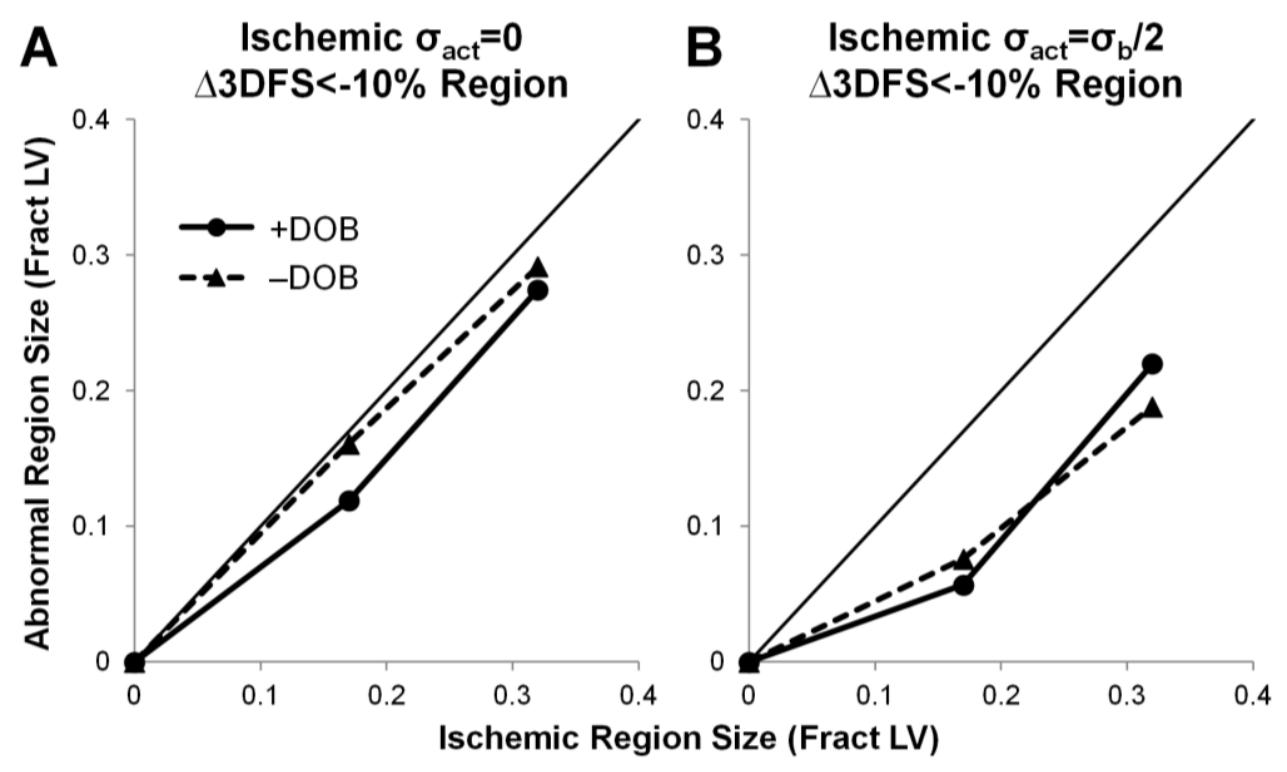

Figure 3.8 Size of $\triangle$ 3DFS abnormalities

The size of the region with $\triangle 3 \mathrm{DFS}<-10 \%$ is similar to ischemic region size in $\sigma_{\mathrm{act}}=0(\mathbf{A})$ and smaller than ischemic region size during $\sigma_{\mathrm{act}}=\sigma_{\mathrm{b}} / 2(\mathbf{B})$, but the abnormality size is similar for both $-\mathrm{DOB}$ and $+\mathrm{DOB}$. 

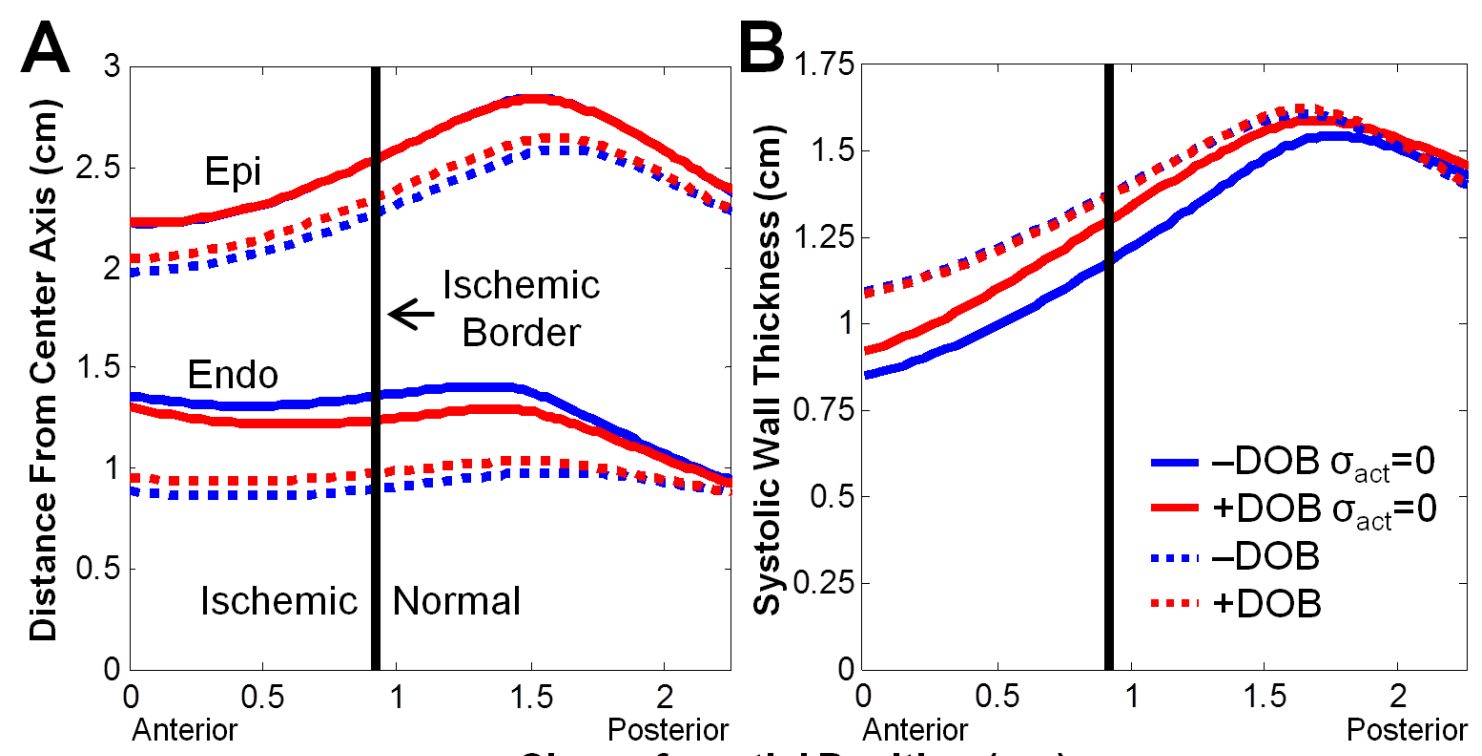

Circumferential Position $(\mathrm{cm})$

Figure 3.9 Detailed view of border zone geometry

A close-up of short axis geometry shows the endocardial and epicardial borders, measured as the distance from the LV center axis, along a circumferential line at the ischemic border. A Small ischemic region with $\sigma_{\text {act }}=0$ at ES shows bulging compared with control (solid vs. dotted lines), and more wall thinning in -DOB (blue) compared with $+\mathrm{DOB}(\mathrm{red})(\mathbf{A})$. Wall thickness in this same region shows an extended region of thinning in -DOB (blue) compared with control, while wall thickness in +DOB (red) does not separate from control until inside the ischemic region (B). 


\section{Comparison of Quantitative Wall Motion Analysis and Strain for Detection of a Critical Stenosis with Three-Dimensional Dobutamine Stress Echocardiography}

\subsection{Introduction}

Dobutamine stress echocardiography (DSE), one of the most commonly-used methods for diagnosing coronary artery disease (CAD), suffers from relatively low interobserver agreement ${ }^{15}$ due to the qualitative nature of diagnosis and its reliance on the expertise of the cardiologist. ${ }^{16}$ The use of quantitative measures is expected to improve accuracy and objectivity of echo image analysis ${ }^{68}$ and numerous methods for measuring regional wall motion, ${ }^{59,60}$ strain, ${ }^{63,64}$ and strain rate ${ }^{62,66}$ during DSE have been developed for implementation in two-dimensional (2D) echo. However, the reproducibility of quantitative measures in 2D echo has been limited by angle dependence, through-plane motion, variation between vendors, and difficulty in defining a center axis for fractional shortening measurement that is not affected by ischemic bulging. ${ }^{61,116,117}$ Because of these issues, no quantitative measurement is currently considered ready for routine clinical use for diagnosis of CAD during DSE. ${ }^{68}$

DSE using three-dimensional (3D) echocardiography (3DE) has been shown to be diagnostically equivalent to $2 \mathrm{D}$ echo, ${ }^{18}$ and $3 \mathrm{DE}$-based measures of mechanical function may overcome many of the limitations of 2D measures. Previously, our group developed a measure of wall motion from 3DE called three-dimensional fractional shortening (3DFS), and showed that 3DFS could be used to detect wall motion 
abnormalities in a finite element model of ischemia ${ }^{78}$ and during transient coronary occlusion in dogs. ${ }^{77}$ Other groups have developed methods for measuring myocardial strain from 3DE using speckle tracking, ${ }^{75}$ and have proposed area strain as a measure that takes advantage of the 3D data and is more sensitive to mechanical abnormalities than circumferential or longitudinal strain. ${ }^{76}$ Various studies have suggested that circumferential strain ${ }^{65,93}$ and peak systolic strain rate ${ }^{66,67}$ are effective for detecting wall motion abnormalities from $2 \mathrm{D}$ stress echo, while the 3DE-based measures area strain and 3DFS have not been evaluated for use during stress testing.

Developing quantitative 3D measures for detecting mechanical abnormalities that indicate CAD during stress testing is one of the most challenging applications of $3 D E$ due to the increased heart rate and relatively low frame rate of 3DE. However, if these technical challenges are overcome, the additional data from 3DE could make quantitative diagnosis of $C A D$ during stress testing more accurate and feasible for clinical use. If this $3 D$ data is available, which measure can best detect CAD that is severe enough to require treatment with catheterization? The modeling results described in Chapter 3 indicate that the $3 \mathrm{D}$ measure area strain is more sensitive to force generation in a region of demand ischemia than $2 \mathrm{D}$ strain measures. In this modeling study the size of ischemic region and amount of force generation could be easily controlled. However, severe stenoses cause regions of demand ischemia during stress testing which display varying size, severity of flow reduction, and severity of force reduction. Therefore, we sought to test these modeling results experimentally in a large animal model, where mechanical measures can be made accurately and the size and location of the ischemic region can be measured, before testing them clinically. The goal of this study was to evaluate the effectiveness of 3DFS for detecting critical stenoses during DSE, and to compare 3DFS to area, circumferential, and longitudinal strain and 
peak systolic strain rate in the ischemic region measured by sonomicrometry, a goldstandard measure of myocardial deformation. We hypothesize that the 3D measures 3DFS and area strain will be more effective than 2D strain or strain rate measures in detecting critical stenoses during DSE.

\subsection{Methods}

\subsubsection{Experimental Protocol}

Eleven dogs were studied using surgical methods and instrumentation described in Chapter 2. A standard dobutamine stress protocol was performed on each animal both without (CTRL) and with (STEN) a critical stenosis in the LAD artery, as diagrammed in Figure 4.1. After surgery and instrumentation, dobutamine was infused in 5-minute dose increments of $5,10,20$ and $30 \mu \mathrm{g} / \mathrm{kg} / \mathrm{min}$. Data acquisition was performed at baseline and at the end of the $30 \mu \mathrm{g} / \mathrm{kg} / \mathrm{min}$ dobutamine dose, including apical 3DE images acquired using a Sonos 7500 ultrasound system (Philips Medical Systems, Andover, MA). A silicone gel standoff (Aquaflex, Parker Laboratories, Fairfield, NJ) was placed between the $x 4$ ultrasound probe and the apex of the heart, and full volume images were acquired over four beats. Radiolabeled microspheres (diameter $15 \mu \mathrm{M}$ labeled with either Sr-85, Nb-95, and Sc-46, Perkin-Elmer, Boston, MA) were injected into the left atrial catheter at baseline and at each maximum dobutamine dose. After the maximum dobutamine dose, dobutamine infusion was stopped and cardiac function gradually returned to baseline. After 45 minutes, data acquisition was performed and a critical LAD stenosis was set as described previously. ${ }^{81}$ The LAD snare ligature was tightened as much as possible without reducing flow, and the hyperemic response was measured by occluding the LAD for 10 seconds and observing the peak hyperemic flow that followed. 
The snare ligature was then adjusted so that the reactive hyperemic response was abolished. After setting the stenosis, the dobutamine stress protocol, data acquisition, and microsphere injection were repeated. Methods for strain and regional blood flow analysis are detailed in Chapter 2. Measures were evaluated on their ability to separate critical stenoses with coronary flow reserve $(\mathrm{CFR})<1.1$ from $\mathrm{CTRL}$ and STEN with CFR $>1.1$.

3DE images were segmented and 3DFS was measured using methods detailed in Chapter 2. 3DFS during CTRL peak stress averaged across all dogs for each location is displayed on a Hammer projection map in Figure 4.2. On average, 3DFS steadily increases from the base to the apex; any measure of abnormal wall motion must therefore detect differences from this pattern. To account for this inhomogeneity, three different methods of normalizing regional 3DFS to compute $\triangle 3 D F S$ were evaluated: 1 ) subtract the average peak stress 3DFS during CTRL at each location, 2) subtract the average resting 3DFS during CTRL at each location, and 3) subtract individual resting 3DFS for that subject at each location. These three options were chosen because they are clinically available. Average resting or peak stress 3DFS may be measured in volunteers without $\mathrm{CAD}$, and baseline images can be acquired for each individual before dobutamine infusion. Each measure was evaluated for sensitivity and specificity of separating $\mathrm{CFR}<1.1$ from $\mathrm{CFR}>1.1$, where the presence of an abnormal $\triangle 3 \mathrm{DFS}$ region larger than $5 \%$ of the LV surface in an experiment with a critical stenosis $(C F R<1.1)$ was defined as a positive test. These ROC curves are displayed in Figure 4.3. Normalization by average stress CTRL 3DFS was the most sensitive and specific for detecting a critical stenosis, so $\triangle$ 3DFS was defined as 3DFS during peak stress for each experiment minus 3DFS during CTRL peak stress averaged for all dogs. The size of a $\triangle 3 D F S$ abnormality was computed as the fraction of the surface area of the LV with $\triangle 3 D F S$ below a 
threshold determined using ROC analysis. For comparisons with strain, $\triangle 3 \mathrm{DFS}$ in the center of the perfusion defect (PD), as defined by regional flow measured by microspheres, was measured by averaging $\triangle$ BDFS in the locations of the tissue blocks containing the sonomicrometer crystals.

\subsubsection{Statistical Analysis}

All values are reported as mean $\pm \mathrm{SD}$. Changes in hemodynamic variables between CTRL and STEN were assessed using a paired t-test. Linear regression was performed using Microsoft Excel 2007 Data Analysis ToolPak, and overall significance of the regression was calculated using the F-test statistic. Receiver operating characteristic

(ROC) curves $^{91}$ were used to compare the diagnostic utility of different measures of regional mechanics by measuring the optimal sensitivity and specificity and the area under the curve $(A \cup C)$ for separating experiments in which $C F R<1.1$ from $C F R>1.1$. Significance of ROC curves for detecting $C F R>1.1$ was calculated with unpaired analysis in GraphPad Prism software (GraphPad Software, San Diego, CA).

\subsection{Results}

A total of 22 dobutamine stress tests were performed, with one CTRL and one STEN in each dog. One CTRL experiment was excluded because the 3DE image quality during peak stress was poor. Hemodynamic data acquired at baseline and peak stress for CTRL and STEN are summarized in Table 4.1. Heart rates and volumes were very similar during peak stress for CTRL and STEN, while systolic pressure and LAD flow were smaller during STEN as expected. Average CFR in the LAD was $1.08 \pm 0.49$ during STEN compared with $3.23 \pm 1.61$ during $C T R L$, and average PD size during 
STEN was $26 \pm 15 \%$ of LV mass, ranging from 2 to $47 \%$. Of the 11 STEN experiments, 8 had CFR $<1.1$. In these experiments, average CFR was $0.86 \pm 0.15$ and average PD size was $31 \pm 13 \%$, ranging from 15 to $47 \%$.

\subsubsection{Detecting Critical Stenoses}

Wall Motion Analysis

A Hammer map of the average 3DFS during CTRL peak stress is shown in Figure 4.2, where each location on the LV surface represents the average 3DFS in that location over 10 dogs. The mean standard deviation in 3DFS over all locations was $0.12 . \Delta 3 D F S$ maps were produced for each STEN image at peak stress. The optimal threshold determined by ROC analysis for detecting regions of abnormal $\triangle 3 D F S$ in experiments with $\mathrm{CFR}<1.1$ and not in those with $\mathrm{CFR}>1.1$, including $\mathrm{CTRL}$ and three STEN experiments, was $\triangle$ BDFS $<-0.275$. With this threshold, the presence of an abnormal $\triangle 3 D F S$ region indicated the presence of a critical stenosis with $88 \%$ sensitivity and $100 \%$ specificity (Table 4.2 ). As $\triangle 3$ DFS was measured over the entire LV surface, the size and location of a region with decreased wall motion should provide information on the size and location of the PD. An example comparing the PD and abnormal $\triangle 3 \mathrm{DFS}$ regions in a typical dog is shown in Figure 4.4A. The abnormal $\triangle$ BDFS region is smaller but contained within the PD. Across all STEN experiments, $97 \%$ of locations within the abnormal $\triangle 3 D F S$ region were also contained in the PD. In STEN cases with CFR $<1.1$, the size of the abnormal $\triangle 3$ DFS region correlated well with the PD size (Figure 4.4B).

\section{Comparison of Wall Motion and Strain}

The efficacy of detecting a critical stenosis with $\triangle 3$ DFS was compared to area, circumferential, and longitudinal strain and peak systolic strain rate measured by 
sonomicrometry. ROC analysis was used to determine the sensitivity and specificity of each measure for separating CFR $<1.1$ from $C F R>1.1$. Figure 4.5 shows $R O C$ curves for all six measures. All curves were significant $(p<0.05)$ for detecting CFR>1.1. $\triangle 3 D F S$, area strain, and circumferential strain and strain rate showed similar sensitivity, specificity, and AUC, while longitudinal strain and strain rate appeared to be less specific (Figure 4.4, Table 4.2).

\subsubsection{Flow-Function Behavior}

Because both the size of an ischemic region and the severity of the flow restriction induced by stress could affect regional mechanics, linear regression was used to test the individual effects of PD size and CFR on $\triangle 3 D F S$ and strain measured in the center of the PD region during STEN. All cases with CFR $<1.5$ were included in the fits, including those where the stenosis was not critical, to evaluate each measure in cases with CFR near the critical stenosis definition. Fit quality, coefficient magnitudes, and coefficient significance are displayed in Table 4.3. PD size was expressed as a fraction of LV mass for regression. CFR ranged from 0.75 to 2.42 , and PD size from 0.02 to 0.47 . $\triangle 3$ DFS regression was significant both with CFR and with PD size as the predictor variable. Surprisingly, area strain regression was only significant with PD size as the predictor variable, not CFR. Regressions of circumferential and longitudinal strain and strain rate with were not significant with CFR or PD size. Figure 4.6 shows more detail of this flowfunction behavior. Area strain displays the expected exponential flow-function behavior when both STEN and CTRL are plotted together (Figure 4.6A). When focusing only on the cases where CFR $<1.5$ and regressions were significant (Figure 4.6B-D), the greatest observed slope represented the change in $\triangle 3$ DFS with PD size. 


\subsection{Discussion}

In this study we evaluated the effectiveness of $\triangle 3$ DFS for diagnosing critical stenoses during cardiac stress testing, and compared the results to strain measured by sonomicrometry. Our results show that $\triangle 3$ DFS can be used to detect a critical stenosis with excellent sensitivity and specificity as well as to measure the size and location of a PD, and is influenced by both CFR and PD size. In comparison, area strain displayed similar diagnostic utility to $\triangle 3$ DFS while longitudinal strain and strain rate did not perform as well. One surprising finding, as mechanical function has traditionally been considered a function of blood flow, is that PD region size accounted for more of the variation in these measures of mechanical function than CFR.

\subsubsection{Wall Motion Analysis}

$\triangle 3 D F S$ showed promise in this study for detecting CAD and quantifying the size of the PD during stress testing. The presence of a $\triangle 3$ DFS abnormality indicated a critical stenosis with $88 \%$ sensitivity and $100 \%$ specificity. In comparison, the standard clinical 2D stress echocardiogram has been shown to detect angiographically-significant CAD with $80 \%$ sensitivity and $86 \%$ specificity. ${ }^{23}$ Measuring function over the entire LV surface also allows quantification of the size, location, and magnitude of a mechanical abnormality. One of our original goals in developing the 3DFS measure was to quantify the size and location of an ischemic region, and we previously demonstrated close correlation between the size of an ischemic region and the size of a wall motion abnormality in both finite element modeling ${ }^{78}$ and during experimental coronary occlusion. ${ }^{77}$ In the present study, we found that the size of a $\triangle$ 3DFS abnormality also 
correlates well with PD size during stress testing, and that regions identified as abnormal by $\triangle$ BDFS are almost always within the PD.

However, these regions of abnormal wall motion were always smaller than the perfusion abnormality. Perfusion abnormalities are known to precede mechanical abnormalities at lower doses of dobutamine and have been reported to be larger than mechanical abnormalities during stress testing. ${ }^{98}$ In addition, $\triangle 3$ DFS abnormality size was smaller than the region of demand ischemia in our modeling results in Chapter 3. Therefore, the observed difference between functional abnormality size and perfusion abnormality size is to be expected. However, conclusions on abnormality size both here and in prior studies are dependent on the chosen cutoff threshold. If a threshold is chosen for high specificity, as a consequence it may measure a smaller abnormality. This is especially true during peak stress, as our models predict a less severe $\triangle$ 3DFS abnormality in regions of demand ischemia with some residual force generation.

In addition, our prior modeling and experimental studies of supply ischemia ${ }^{77,78}$ suggest that tethering to adjacent healthy myocardium may limit detection of small ischemic regions by wall motion-based approaches. The two smallest ischemic regions with CFR $<1.1$ in this study were $15 \%$ of LV mass, and our analysis detected one of the two, along with all larger ischemic regions. Based on these results, we cannot yet reach any definitive conclusions about the performance of $\triangle 3 D F S$ for detection of demand ischemia affecting $<15 \%$ of LV mass. Overall, our results suggest $\Delta 3 D F S$ has excellent potential not only for identifying the presence of a critical stenosis during stress testing but also for increasing the amount of diagnostic information obtained from a stress test by taking advantage of the additional data collected in a full volume 3D echocardiogram. 


\subsubsection{Strain Analysis}

Area strain measured in the center of the PD correlated well with PD size and could detect critical stenoses better than circumferential or longitudinal strain. This was expected, as area strain describes deformation in both the circumferential and longitudinal directions and previous studies have shown area strain to be more sensitive to changing mechanics than circumferential or longitudinal strain. ${ }^{76}$ In comparison, even using sonomicrometry, a gold standard technique with low noise and error, circumferential and longitudinal strain correlated poorly with CFR and PD size during restricted flow reserve. Previous studies have shown conflicting results in comparing circumferential and longitudinal strain for diagnosis of dobutamine-induced ischemia; some studies show circumferential strain to be superior to longitudinal, ${ }^{93}$ while others claim they are diagnostically equivalent. ${ }^{65,94}$ Longitudinal strain was often used in the past because it was easier to measure with tissue Doppler imaging than circumferential strain. A detailed study of strains measured with radiopaque beads in dogs during brief coronary occlusion at rest showed that the amount of endocardial and midwall dyskinesis after 5 minutes of ischemia is similar in the longitudinal and circumferential directions, and the change in endocardial strain from control to ischemia was $0.19 \pm 0.08$ in the circumferential direction and $0.16 \pm 0.06$ in the longitudinal direction. ${ }^{34}$ Therefore, some of the disagreement in the literature may be due to differences in ability to measure these two strain components with various techniques, rather than to inherent differences in mechanics in the circumferential and longitudinal directions. This further emphasizes the advantage of using area strain, which captures changes in both circumferential and longitudinal strain, when available.

Strain rate measured by tissue Doppler imaging is another commonly-used quantitative measure of regional mechanics, ${ }^{118}$ and has been evaluated for detecting 
critical stenoses during stress testing. ${ }^{66,94,109}$ Longitudinal strain rate is most often reported because it is the easiest parameter to measure with tissue Doppler. However, in this study we found that peak systolic circumferential strain rate was more specific for detecting CFR $<1.1$ than longitudinal strain rate. In fact, Figure 4.5 shows that the ROC curves for circumferential strain and strain rate are very similar, as are longitudinal strain and strain rate. Circumferential peak systolic strain rate showed less variation during CTRL peak stress than the longitudinal direction, and was also observed to be more sensitive to ischemic force generation than the longitudinal direction in the Chapter 3 modeling observations. These results indicate that while peak systolic strain rate does not exhibit better sensitivity or specificity than strain for detecting critical stenoses, if strain rate is used then measuring in the circumferential direction is more effective for detecting critical stenoses during stress testing than the longitudinal direction.

\subsubsection{Flow-Function Relationships}

Traditionally, regional mechanical dysfunction during ischemia has been described as a

function of the change in blood flow from baseline during both supply ${ }^{33,119}$ and demand ${ }^{120}$ ischemia. However, in this study we found that area strain in the center of the ischemic region correlates better with PD size than CFR during STEN. We therefore checked for potential artifacts that could contribute to this unexpected result. For example, if the sonomicrometers were not located exactly in the center of the PD region, blood flow and mechanical function could have been better near the sonomicrometers in animals with smaller PDs. We therefore plotted MFR in the tissue blocks containing the sonomicrometers against PD size to see if MFR would decrease as PD size increases. However, MFR in this region showed no relationship with PD size $\left(R^{2}=0.042\right)$. We also 
tested the sensitivity of our conclusions to the specific choice of MFR cutoff used to separate normally and abnormally perfused regions when defining PD size. Average PD size increased or decreased with the changing threshold, but the quality of correlation between PD size and AAF did not change. We conclude that during dobutamine stress, PD size is an important determinant of mechanical behavior and may be even more important than severity of blood flow restriction.

\subsubsection{Application to Clinical Diagnosis}

Testing $\triangle$ 3DFS in an animal study allows us to compare our results to gold-standard measurements of perfusion and strain; the next step is to test these results clinically. $\triangle$ BDFS can be measured clinically with the same methods used in this study. $\triangle 3 D F S$ is based only on the endocardial segmentation of ED and ES, which has been validated for

measuring volumes against $\mathrm{MRI}$ in a multicenter trial. ${ }^{92}$ Regional wall motion is more susceptible to segmentation error than volumes, as normally-distributed segmentation errors can cancel each other out when measuring global volume, but these challenges were also present in the current study. In addition, semi-automatic segmentation software for 3DE continues to improve in both accuracy and ease of use, reducing the time required for clinical application of $\triangle 3$ DFS. ${ }^{121,122}$

Error in measuring strain, on the other hand, is much higher when measured clinically with speckle tracking than in the sonomicrometry data used in this study. Further, the accuracy of 3D speckle tracking measurement is highly dependent on frame rate, ${ }^{123}$ and therefore speckle tracking during peak stress is more prone to error than at rest. To get a rough idea of how strain measured from speckle tracking would compare to $\triangle 3 D F S$ in this study, we performed a Monte Carlo simulation of expected speckle 
tracking strains based on our sonomicrometry measurements. We used the BlandAltman bias and standard deviation values available in literature for $2 D^{63}$ and $3 D^{75}$ speckle tracking for circumferential and longitudinal strain and 3D speckle tracking for area strain ${ }^{76}$ vs. sonomicrometry to generate a probability distribution of speckle tracking measurements for each sonomicrometry measurement performed in the study. Table 4.4 details the average ROC analysis results from 1000 simulations. Due to higher error in $3 D$ speckle tracking, we find that circumferential strain measured from $2 \mathrm{D}$ echo has the highest AUC for detecting CFR $>1.1$ in this analysis, followed closely by area strain. With the addition of this estimated error, AUC for area strain and circumferential strain are not different than that for $\triangle 3$ DFS in this study, while longitudinal strain still performs less well. This reinforces our observation that even though strain provides more local information on myocardial deformation, $\triangle 3$ DFS may perform just as well for diagnosing critical stenoses.

\subsubsection{Comparison to Model Results}

These wall motion and strain results provide a point of comparison for the model results described in Chapter 3. Examining these experimental and model results in tandem shows the advantage of using models to inform experiments and vice versa. These experiments confirm the model prediction that area strain is the most sensitive measure relative to measurement error for detecting ischemic region force generation, followed closely by 3DFS and circumferential strain. The model was also correct in predicting the advantage of peak systolic strain rate in the circumferential direction over longitudinal for measuring ischemic force generation. Longitudinal strain was not as sensitive to ischemic force generation in the experiments as in the model. The difference between 
the average measure for $\mathrm{CFR}>1.1$ and $\mathrm{CFR}<1.1$ for area strain, circumferential strain, and longitudinal strain in these experiments was $0.19,0.13$, and 0.06 , respectively, while the differences in the model were $0.15,0.07$, and 0.08 respectively. The model underestimated the change in area strain and circumferential strain while slightly overestimating the change in longitudinal strain.

The regressions of each measure as a function of CFR and PD size also provide a point of comparison to the model study. While 3DFS and area strain were both more sensitive to PD size during peak stress than other measures, overall all measures were much more sensitive to ischemic force generation than size in the model. In contrast, PD size described more variation in area strain and $\triangle 3$ DFS than CFR in these experiments. There are important differences in the model and these experiments which may affect this comparison of the importance of CFR and PD size. The ischemic regions were completely transmural and homogeneous in the model, while the experimental regions of demand ischemia were likely not fully transmural. The microsphere analysis method used here was designed to maximize the resolution of flow measurement over the LV surface, and as a result transmural variation was not captured. There are likely blocks both inside and outside of the PD region, and near the edge especially, with reduced endocardial flow and normal epicardial flow. It is possible that in dogs that had more collateral flow, CFR could have been reduced significantly without impacting mechanical function because the flow abnormality was non-transmural. In experiments with matched CFR, PD size would likely be larger for abnormalities that were more transmural. Further modeling of non-transmural ischemic regions would help to determine the effect of ischemic region transmurality on regional mechanical function. Joint analysis of the model and experiments was helpful for confirming the results that are the same and can lead to further investigation to understand the causes of differences. 


\subsubsection{Sources of Error and Limitations}

Most of the error in 3DFS measurement in this study arises from variation in manual endocardial segmentation. The semiautomatic segmentations used in this study required heavy manual editing, and images with abnormal wall motion required more editing than normal images. Another potential source of error is the lingering effect of the first stress test on the second stress test in each animal. However, we found that the difference in function between the first baseline and the second was negligible. The mean difference between the average 3DFS at each location on the LV surface at the first baseline and the second was 0.002 , and LAD flow was not significantly different between baselines. Heart rate and LV volumes and pressures at ED were not significantly different $(p<0.05)$, while LV pressure was only slightly depressed and LV volume slightly larger in the second baseline compared to the first. Therefore, we conclude that wall motion, coronary flow, and hemodynamics recovered adequately between the two stress tests.

One potential limitation of the analysis outlined here relates to defining $\triangle 3 D F S$ relative to the average response to DSE in normal hearts. Clinical application of $\triangle 3 D F S$ as defined here will require establishing the average response to 3DFS at peak stress in the absence of ischemia as a baseline for comparison. The size, shape, and function of the canine hearts in this study were fairly homogenous, so 3DFS in the CTRL peak stress images used for normalization had low variability. Patients who undergo DSE and prove to have no clinically significant coronary artery disease likely will display more 3DFS variability; if this variability proves limiting in defining a uniform threshold for detecting critical stenoses clinically, an alternate approach would be to focus on the regional changes in 3DFS between baseline and stress in individual patients, an

approach we have taken in prior animal studies. ${ }^{77}$ However, this technique resulted in lower sensitivity and specificity in $\triangle 3$ DFS in this study (Figure 4.3), so we predict that 
normalization by average peak stress 3DFS will be most effective clinically. This question will be addressed in Chapter 6 by measuring the variability in peak stress 3DFS in a clinical population at low risk for CAD. A second limitation of this study is that it was powered to test the ability of individual mechanical measures to separate cases with CFR $<1.1$ from those with CFR $>1.1$ using ROC analysis, but not to test for statistical differences between mechanical measures in making that determination. From an ROC power analysis ${ }^{124}$ we estimate that $n=80$ experiments would be necessary to statistically differentiate between the measures with largest and smallest AUC in this study, area strain and longitudinal strain. Our data therefore should not be used to draw definitive conclusions regarding the relative ability of various strain measures to detect critical stenoses. Finally, compared to the way these measures would be used in clinical practice, our comparison of $\triangle 3 \mathrm{DFS}$ and strain likely placed strain at a disadvantage for predicting PD size and 3DFS at a disadvantage for detecting the presence of a critical stenosis. We measured $\triangle 3$ DFS over the entire LV surface but strain only at one location in the LAD perfusion region. If strain were measured over the entire LV using 3D speckle tracking, it might also provide useful information on the size, location, and severity of a wall motion abnormality during stress testing. On the other hand, we measured strain by sonomicrometry, which is the invasive gold standard for measuring strain, giving strains a potential advantage over $\triangle 3$ DFS in identifying ischemic regions in this study. Despite these limitations, our data and Monte Carlo simulation suggest that the sensitivity and specificity of $\triangle 3 D F S$ is comparable to $2 D$ circumferential strain or $3 D$ area strain measured with current speckle tracking technology.

The scope of this study was limited to evaluating mechanical measures in the case of single vessel critical stenoses. The optimal thresholds for this criteria resulted in high specificity in detecting critical stenoses but lower sensitivity to mild ischemia, as 
illustrated in Figure 4.4B. These results are similar to the higher specificity and lower sensitivity of stress echocardiography with visual diagnosis of wall motion abnormalities compared with perfusion imaging, ${ }^{23}$ and likely indicate a limitation of wall motion for sensitivity to CAD. An ideal clinical measure would also be sensitive to wall motion abnormalities caused by multi-vessel disease or microvascular disease. While these applications have yet to be tested, defining $\triangle$ BDFS relative to a standard normal response in patients without ischemia instead of focusing on regional differences in the stress response could have advantages in detecting multi-vessel disease.

\subsection{Conclusion}

The present study demonstrates that quantitative wall motion analysis using $\triangle 3$ DFS can be used to detect a critical stenosis during DSE with sensitivity and specificity similar to 3D strain. In addition, $\triangle 3$ DFS provides potentially useful new information on the size and location of a PD. This quantitative analysis method shows promise for improving and quantifying diagnosis of CAD from cardiac stress testing. 
Table 4.1 Hemodynamic parameters

\begin{tabular}{lccc}
\hline & Baseline & CTRL Stress & STEN Stress \\
\hline HR $(\mathrm{bpm})$ & $97 \pm 13$ & $171 \pm 23$ & $177 \pm 29$ \\
EDV $(\mathrm{mL})$ & $36.7 \pm 8.5$ & $36.4 \pm 10.6$ & $39.1 \pm 6.5$ \\
ESV $(\mathrm{mL})$ & $17.5 \pm 5.3$ & $11.0 \pm 2.8$ & $14.3 \pm 6.8$ \\
EF $(\%)$ & $52.5 \pm 6.8$ & $69.2 \pm 6.0$ & $63.8 \pm 15.0$ \\
CO $(\mathrm{L} / \mathrm{min})$ & $1.85 \pm 0.46$ & $4.19 \pm 0.91$ & $4.24 \pm 0.70$ \\
EDP $(\mathrm{mmHg})$ & $8.1 \pm 3.1$ & $8.4 \pm 2.9$ & $9.1 \pm 2.3$ \\
ESP $(\mathrm{mmHg})$ & $77.9 \pm 8.5$ & $104.8 \pm 13.6$ & $94.4 \pm 20.2^{*}$ \\
LAD flow $(\mathrm{mL} / \mathrm{min})$ & $40.5 \pm 23.1$ & $108.9 \pm 36.3$ & $20.4 \pm 7.6^{*}$ \\
\hline
\end{tabular}

Values are mean $\pm S D$ ( $n=10$ for CTRL peak stress, $n=11$ otherwise). $H R=$ heart rate; $\mathrm{EDV}=$ end-diastolic volume; $\mathrm{ESV}=$ end-systolic volume; $\mathrm{EF}=$ ejection fraction; $\mathrm{CO}=$ cardiac output; EDP = end-diastolic pressure; ESP = end-systolic pressure; LAD = left anterior descending artery.

* Indicates significantly different from CTRL by paired t-test. 
Table 4.2 ROC characteristics of measures of regional mechanics

\begin{tabular}{lccc}
\hline & Sensitivity & Specificity & AUC \\
\hline$\Delta 3$ DFS & $88 \%$ & $100 \%$ & 0.885 \\
Area Strain & $88 \%$ & $100 \%$ & 0.982 \\
Circ Strain & $88 \%$ & $92 \%$ & 0.929 \\
Long Strain & $88 \%$ & $71 \%$ & 0.875 \\
Peak SR Circ & $88 \%$ & $86 \%$ & 0.946 \\
Peak SR Long & $88 \%$ & $79 \%$ & 0.875 \\
\hline
\end{tabular}

3DFS $=$ Three-dimensional fractional shortening, $S R=$ strain rate, $\mathrm{ROC}=$ receiver operating characteristic, $\mathrm{AUC}=$ area under the ROC curve. 
Table 4.3 Linear regression parameters and significance

\begin{tabular}{lcccccc}
\hline & \multicolumn{3}{c}{ CFR } & & \multicolumn{3}{c}{ PD size } \\
$Y$ & $R^{2}$ & coeff & $p$-value & $R^{2}$ & coeff & $p$-value \\
\hline S3DFS & 0.71 & 0.50 & 0.021 & 0.79 & -0.84 & 0.006 \\
Area strain & 0.63 & -0.29 & 0.052 & 0.67 & 0.46 & 0.035 \\
Circ strain & 0.50 & -0.17 & 0.139 & 0.53 & 0.28 & 0.113 \\
Long strain & 0.41 & -0.07 & 0.243 & 0.20 & 0.05 & 0.585 \\
Peak SR Circ & 0.50 & -1.68 & 0.144 & 0.40 & 2.03 & 0.257 \\
& & & & & & \\
Peak SR Long & 0.12 & 0.32 & 0.733 & 0.09 & -0.33 & 0.814 \\
& & & & & & \\
\hline
\end{tabular}

3DFS $=$ three-dimensional fractional shortening, $\mathrm{CFR}=$ coronary flow reserve, $\mathrm{PD}=$ perfusion defect. 
Table 4.4 Monte Carlo simulation of speckle tracking strain performance

\begin{tabular}{lcccc}
\hline & Modality & AUC & Sensitivity (\%) & Specificity (\%) \\
\hline Area Strain & 3D & $0.90 \pm 0.06$ & $87 \pm 9$ & $87 \pm 10$ \\
Circ Strain & 3D & $0.85 \pm 0.07$ & $85 \pm 10$ & $83 \pm 9$ \\
Long Strain & 3D & $0.75 \pm 0.09$ & $75 \pm 12$ & $76 \pm 12$ \\
Circ Strain & 2D & $0.92 \pm 0.03$ & $93 \pm 7$ & $88 \pm 4$ \\
Long Strain & 2D & $0.83 \pm 0.05$ & $81 \pm 10$ & $79 \pm 10$ \\
\hline
\end{tabular}


Surgery and

instrumentation

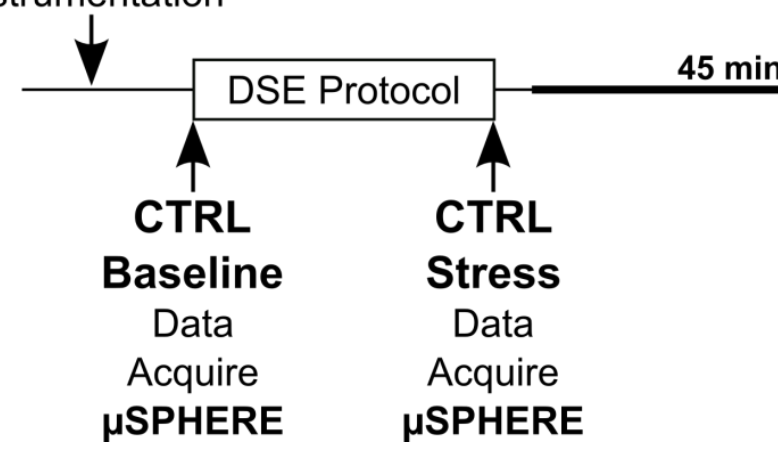

Set critical LAD

stenosis

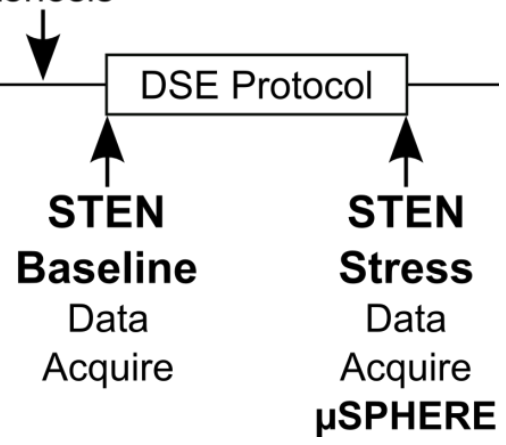

Figure 4.1 Experimental protocol

Dobutamine stress echocardiography (DSE) is repeated with (STEN) and without (CTRL) a critical LAD stenosis. Data, including left ventricular pressure, regional strain, global function, LAD flow, and 3DE images, are collected as indicated. $\mu$ SPHERE indicates microsphere injection for quantification of regional blood flow. 


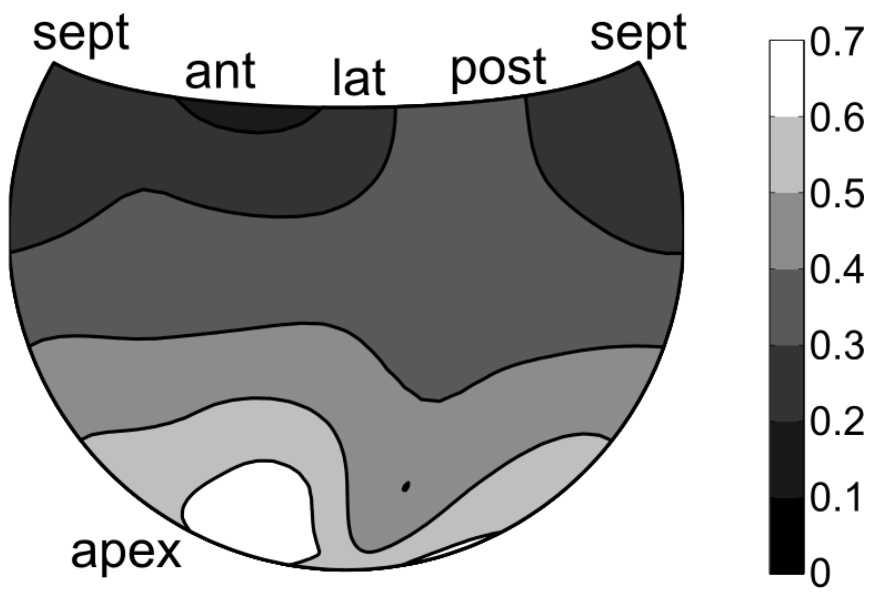

Figure 4.2 Average 3DFS during peak stress CTRL experiments 


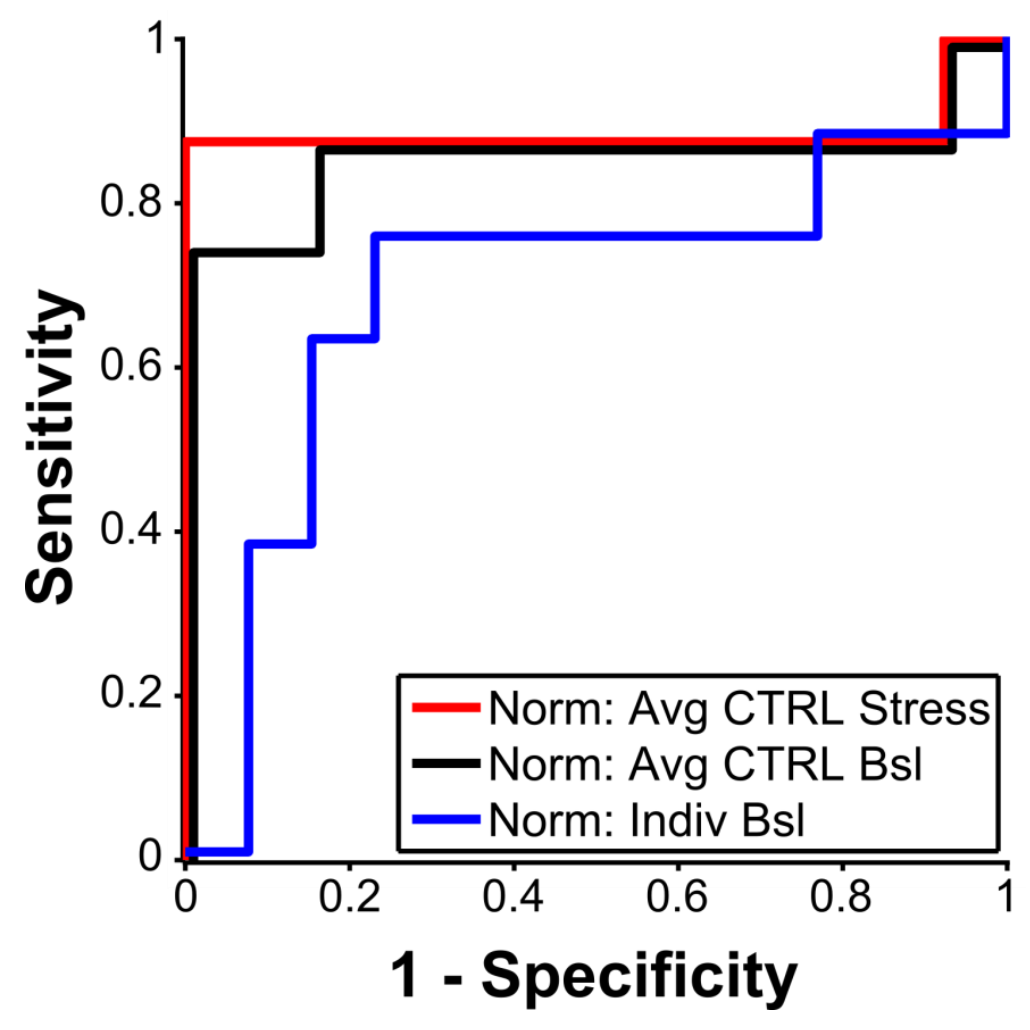

Figure 4.3 ROC analysis of 3DFS normalization methods

ROC analysis is used to compare methods of normalizing 3DFS for sensitivity and specificity in separating CFR $<1.1$ from $\mathrm{CFR}>1.1$. 

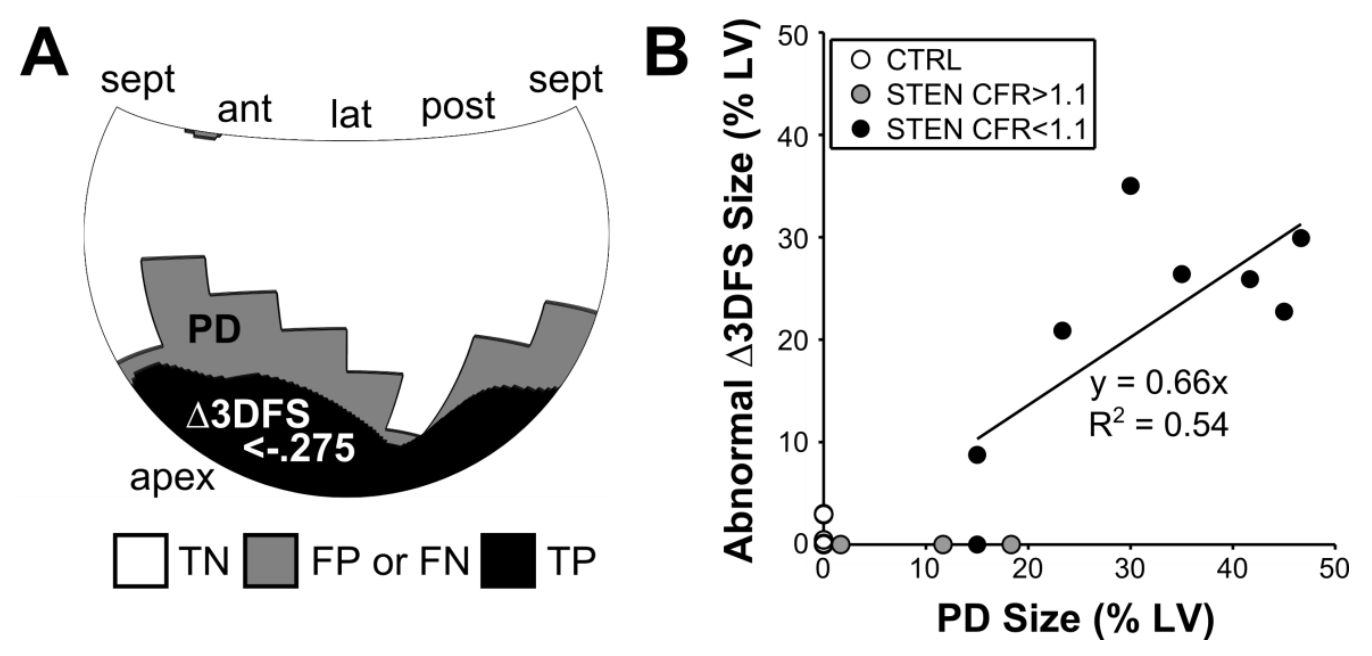

Figure 4.4 Measuring PD size with $\triangle$ 3DFS

By calculating the area of the LV surface with $\triangle 3$ DFS below an optimum threshold, we can measure the size and location of a wall motion abnormality. (A) In a typical example, the area with $\triangle 3$ DFS below the optimal threshold is contained within the PD region determined by microspheres. Regions are classified by a true positive (TP) or true negative (TN) match between the PD region and abnormal $\triangle$ SDFS region, or false positive (FP) or false negative (FN) mismatch. (B) Across all experiments where CFR $<1.1$, the size of the $\triangle 3 \mathrm{DFS}$ abnormality correlates with PD region size. 


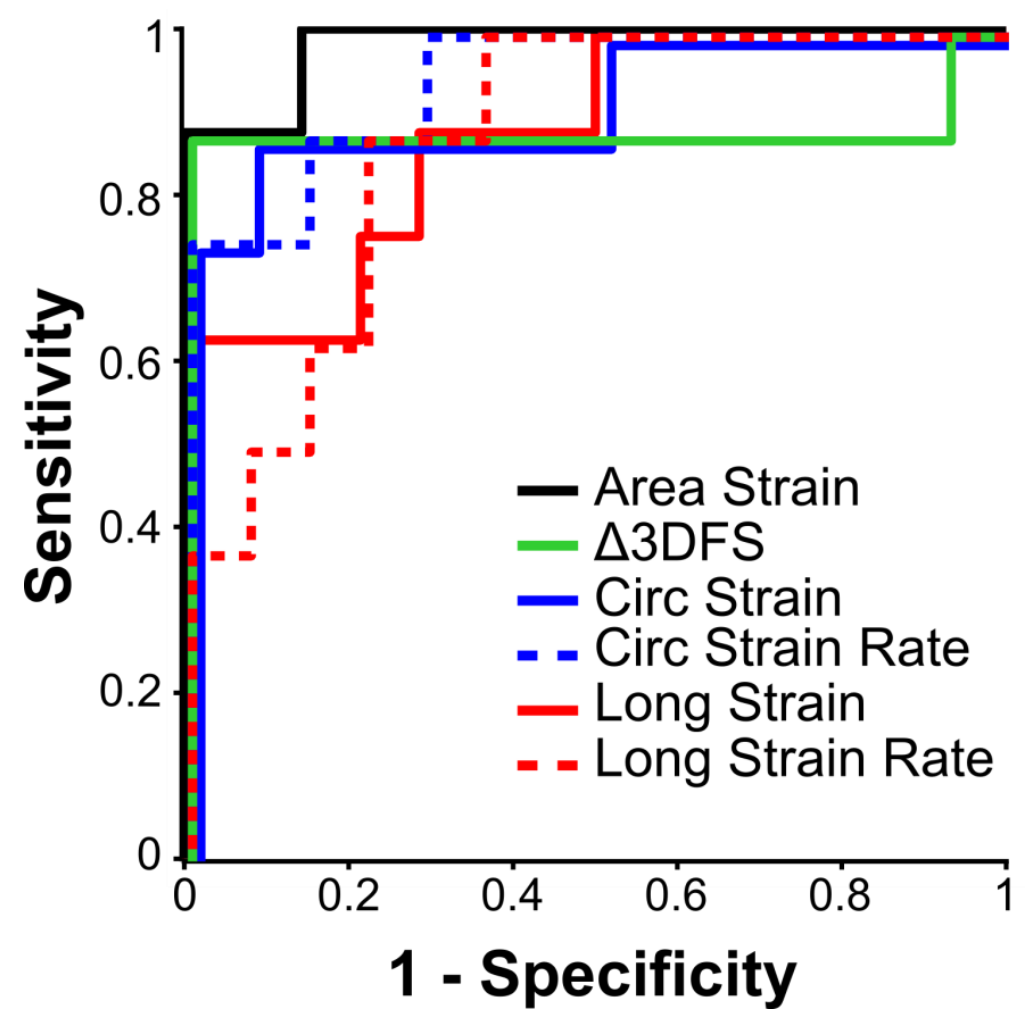

Figure 4.5 ROC analysis for detecting critical stenosis

ROC analysis shows the utility of each measure for separating CFR $<1.1$ from CFR $>1.1$ and CTRL. All curves overlap at sensitivity $<0.6$ and $=1.0$ but are offset slightly for better visibility. 

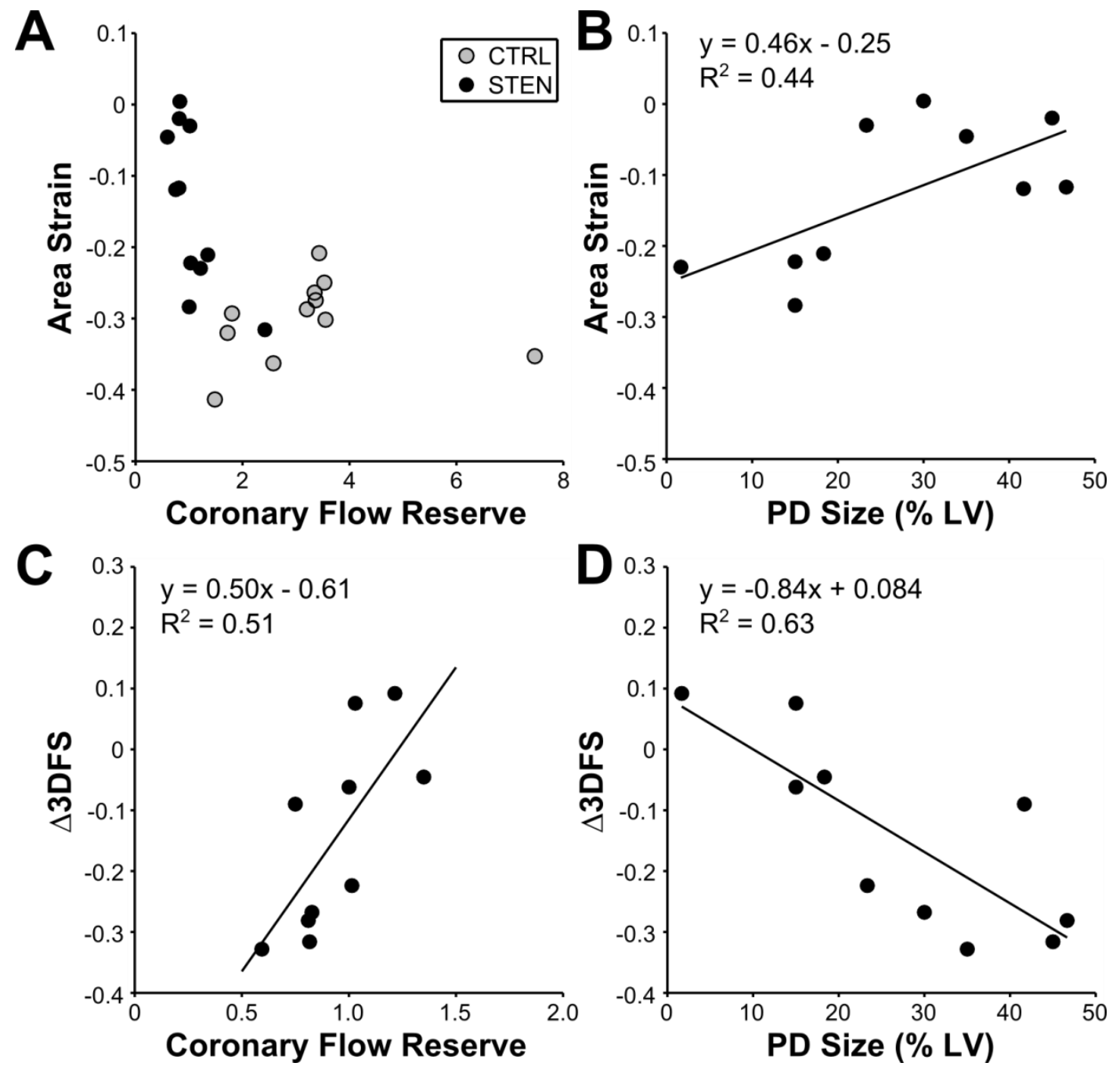

Figure 4.6 Flow-function relationships

Area strain displays the expected exponential flow-function relationship with CFR in both CTRL and STEN (A). When focusing on cases in which CFR $<1.5$, a significant relationship was found between area strain and PD size (B) and $\triangle 3$ DFS and CFR (C) and PD size (D). 


\section{Evaluation of Quantitative Wall Motion Analysis for Distinguishing Between Stiff Infarcts and Acute Ischemia}

\subsection{Introduction}

It is estimated that $20 \%$ of myocardial infarctions (MI) in both middle-aged ${ }^{20}$ and elderly ${ }^{21}$ patients are unrecognized. The presence of an infarct, especially an unrecognized MI that is not present in a patient's records, can make the diagnosis of demand ischemia from stress testing very difficult. Currently, if a wall motion abnormality (WMA) is present at rest and does not worsen during stress it is classified as "fixed", and while this stress test result is not considered normal, fixed WMAs are often assumed to indicate scar untreatable by revascularization. ${ }^{23}$ However, these WMAs caused by old infarcts may either falsely appear to indicate treatable ischemia or mask the presence of viable myocardium. ${ }^{24} \mathrm{~A}$ fixed WMA is easily explained if the patient has a recorded history of MI, but without this history the WMA could indicate an unrecognized MI, acute ischemia, or even another disease that can create fixed WMAs such as left bundle branch block. ${ }^{125}$ A quantitative method for distinguishing between WMAs caused by acute ischemia or WMAs caused by old infarcts would help in classifying these resting WMAs, increasing the specificity of stress testing.

Both ischemic and infarcted regions cause WMAs, as neither contain many contracting myocytes. While an acutely ischemic region behaves similarly to passive myocardium, the infarct stiffens drastically starting about 4 hours after infarction and continues stiffening for weeks. ${ }^{126,127}$ Most of this change is due to increased collagen content in the infarct. ${ }^{126,128}$ The end-systolic (ES) volume is greater in a ventricle with 
acute ischemia than with a stiff infarct of the same size, ${ }^{99}$ as the infarct bulges less during systole. We reasoned that a measure of infarct bulging may be able to detect whether a WMA is caused by an old infarct or new ischemia. Previous work in our lab using a finite element (FE) model of the heart showed that a WMA caused by an infarct appears similar in size to an abnormality caused by a smaller ischemic region, and that a measure of wall motion bulging, called the dyskinesia severity index (DSI), could distinguish between acutely ischemic regions and stiff infarcts. ${ }^{80}$ In this study, we will first verify these model results in a more realistic FE model of the canine heart with either an acutely ischemic region or a stiff infarct that is coupled to a closed circulation model, as described in Chapter 3. Then, we will measure DSI in canines with either experimentallyinduced acute ischemia or healed infarcts. Finally, DSI will be evaluated for use during stress testing in a FE model of dobutamine stress with either a stiff infarct or a region of demand ischemia. We hypothesize that DSI will change with the stiffness of an ischemic region but not with ischemic region size or stress, differentiating between WMAs caused by stiff infarcts in comparison with acute ischemia.

\subsection{Methods}

\subsubsection{FE Modeling}

An FE model of the heart was used to evaluate the performance of DSI in models of acute ischemia and infarction during both rest and stress conditions. The baseline, stress, and ischemic FE models were described in detail and validated in Chapter 3. These models differ from those previously used to test DSI in that ED and ES were not modeled simply as pressure inflations of passive and fully contracted ventricles, but 
through coupling to a closed-circulation model of the entire cardiac cycle. Regions of ischemia or infarction were defined for an example small and large anterior region as described in Chapter 3. Infarcts were modeled by isotropically increasing the passive stiffness of the ischemic region using methods previously described by our lab. ${ }^{129}$ The constitutive properties of passive myocardium in the model are expressed by a transversely isotropic exponential Fung-type strain energy function $W:^{103}$

$$
\begin{aligned}
& W=0.5 * C *\left(e^{Q}-1\right)+0.5 * K * \ln (\mathrm{J})^{2} \\
& Q=b_{f f} E_{f f}^{2}+b_{x x}\left(E_{c c}^{2}+E_{s s}^{2}+E_{c s}^{2}+E_{s c}^{2}\right)+b_{f x}\left(E_{f c}^{2}+E_{c f}^{2}+E_{f s}^{2}+E_{s f}^{2}\right)
\end{aligned}
$$

Where $Q$ is a function of components of the Lagrangian strain tensor $\mathbf{E}$ : fiber strain $\left(E_{\mathrm{ff}}\right)$, cross-fiber strain $\left(E_{\mathrm{cc}}\right)$, radial strain $\left(E_{\mathrm{ss}}\right)$, and shear strains, and $J$ is the determinant of the deformation gradient tensor F. Normal passive canine myocardium was represented with the coefficients $C=0.88 \mathrm{kPa}, b_{\mathrm{ff}}=18.5, \quad b_{\mathrm{xx}}=3.58, b_{\mathrm{fx}}=1.63$, and $K=100 \mathrm{kPa}$. To increase the passive isotropic stiffness of the infarct, active force generation was turned off and $b_{\mathrm{ff}}, b_{\mathrm{xx}}$, and $b_{\mathrm{fx}}$ were increased to 50 , as was used previously by our lab. ${ }^{129}$

To evaluate DSI in the case of a resting WMA, DSI measured from models of supply ischemia (no dobutamine, $-\mathrm{DOB})$ with no ischemic force generation $\left(\sigma_{\mathrm{act}}=0\right)$ was compared to DSI measured from models of infarcts. Both small (17\% of LV) and large (32\% of LV) abnormal regions were analyzed. To evaluate DSI in the case of dobutamine stress, DSI was measured in models of peak dobutamine stress (+DOB) with a severe critical stenosis allowing limited force generation $\left(\sigma_{a c t}=25\right)$. This case was compared to peak dobutamine stress with a stiff infarct region with no force generation. Models were run to steady state, the ED and ES times were selected, and 3DFS was calculated as described in Chapter 3. 


\subsubsection{In Vivo Wall Motion Analysis}

Data were collected from dogs during acute ischemia immediately following coronary occlusion, 48 hours after a 90 minute coronary occlusion and reperfusion, and 8 weeks following reperfusion. Data during acute ischemia were collected in five dogs with four occlusion sites and sizes per dog. In a separate group, infarction data were acquired in 16 dogs at 48 hours and 8 weeks following a 45 -minute occlusion. Wall motion was measured with three-dimensional echocardiography (3DE) during acute ischemia, and with cardiac magnetic resonance (CMR) at 48 hours and 8 weeks, and analyzed to calculate DSI in the WMA region.

\section{Acute Ischemia}

3DE images acquired in five dogs during a previously published study of acute ischemia were included in this analysis. ${ }^{77}$ Briefly, a thoracotomy was performed at the fifth intercostal space, and ligature snares were placed proximal and distal to the main branch of the left anterior descending (LAD) and left circumflex (LCx) arteries. A brief two minute total occlusion was performed at each site in random order, and 3DE images were acquired during each occlusion using a Philips iE33 ultrasound system (Philips Medical Systems, Andover, MA). A total of 20 images were acquired for 4 occlusion sites in 5 dogs, and one image was discarded due to poor quality. Therefore, 19 images of acutely ischemic regions of varying sizes and locations were analyzed. The previouslyreported wall motion analysis for this data set ${ }^{77}$ was used in this study as well. Finally, colored microspheres were infused during ischemia and counted posthumously to measure the size of the ischemic region as a fraction of LV mass, as described in the original study. ${ }^{77}$ 
Infarcts

A subset of dogs from a previously published study ${ }^{130}$ of $\mathrm{Ml}$ were included in this analysis. Briefly, a thoracotomy was performed at the fifth intercostal space, and the LAD artery and surrounding collaterals were occluded for 90 minutes. In the experiment group, an adenosine $A_{2 A}$ receptor agonist was administered intravenously after 60 minutes of occlusion. All ligatures were removed after 90 minutes and the chest was closed, and each dog was imaged with CMR 48 hours and 8 weeks following reperfusion. CMR imaging was performed using a 1.5T clinical scanner (Siemens Avanto). Steady state free precession imaging was performed to generate 6 to 9 short axis slices at the left ventricular (LV) base, mid-level, and apex locations (temporal resolution: $38.6 \mathrm{~ms}$, echo time: $1.4 \mathrm{~ms}$, flip angle: $\alpha=65^{\circ}$, field of view: 20 to $25 \mathrm{~cm}$, spatial resolution: $0.98 \mathrm{~mm}$ per pixel). Images of 12 dogs (6 experiment, 6 control) at 48 hours post-MI and 16 dogs (8 experiment, 8 control) at 8 weeks post-MI were included. Both experiment and control dogs were included, as the $A_{2 A}$ agonist is known to affect reperfusion injury, infarct size, and inflammation, but not the stiffness of a healed infarct. Wall motion analysis methods were the same as those reported in Chapter 2, except that the endocardium was segmented using Siemens Argus software.

Infarct size was measured with late-gadolinium enhanced MR images to view scar size and location. Delayed-enhancement images in locations identical to the cine images were acquired 10 to $15 \mathrm{~min}$ after a bolus injection of $0.2 \mathrm{mmol} / \mathrm{kg}$ gadodiamide (Omniscan, GE Healthcare, Buckinghamshire, United Kingdom). An inversion recovery fast gradient-echo pulse sequence was used for the acquisition. The endocardium, epicardium, and scar were manually segmented from the late-enhanced images using Siemens Argus software, and scar location and transmurality was interpolated over the 
LV surface as described in Chapter 7. Scar size was expressed as a fraction of the LV surface area.

\subsubsection{Wall Motion Analysis}

Fitting and wall motion analysis was completed for all images and FE models as described in Chapter 2, and 3DFS was measured over the entire LV surface. A cutoff of 3DFS $=0.05$ was used to detect regions of akinesis and dyskinesis in all rest models and experiments, and a cutoff of 3DFS $=0.15$ was used in models of peak stress. The size of the region with $3 D F S<$ cutoff is defined as the WMA size. The DSI calculation is illustrated in Figure 5.1. The goal of DSI is to capture the "area under the curve" of 3DFS in the WMA region. DSI was calculated according to Equation 1:

$$
D S I=\frac{\sum_{W M A} \mid 3 D F S-\text { cutoff } \mid / \text { Area }}{\sum_{W M A} \text { Area }}
$$

where the sum was computed for each data point within the WMA region (3DFS<threshold) covering a given Area. DSI was calculated by dividing this value by the size of the WMA to obtain a measure of the average bulging in this region. Differences in ischemic or infarct region size, WMA size, and DSI were evaluated using an unpaired student's t-test, and the sensitivity and specificity of detecting an infarct with DSI was evaluated using receiver operator characteristic $(R O C)$ analysis. 


\subsection{Results}

\subsubsection{Acute Ischemia vs. Infarcts At Rest}

\section{FE Modeling}

Hammer projection maps of 3DFS over the LV surface of models at rest are shown in Figure 5.2. WMAs caused by infarcts appear to be smaller than those caused by acutely ischemic regions of the same size. There is also clearly more bulging in the acutely ischemic regions, evidenced by more negative 3DFS values. As a result, DSI is larger for the acutely ischemic models, indicating more bulging, than in the infarct models. While the WMAs caused by the large infarct and the small ischemic region appear to be of similar size, DSI for the infarct is smaller than the acutely ischemic region. Based on this analysis, DSI shows promise for detecting WMAs caused by infarcts that falsely appear to indicate a smaller region of acute ischemia.

\section{In Vivo Wall Motion Analysis}

Measurements of WMA size and DSI in dogs during acute ischemia, 48 hours after a 45minute occlusion, and 8 weeks after a 45-minute occlusion are shown in Table 5.1. Because both the acutely ischemic data and the 48 hour data were acquired before significant collagen deposition in the infarct, ${ }^{127}$ the 8 week infarct should be much stiffer than the other infarcts. The infarct size at 8 weeks is significantly smaller than 48 hours and acute, and WMA size is significantly different between all groups. DSI during acute ischemia is not different than DSI at either infarct time point, but DSI is significantly smaller at 8 weeks than 48 hours. The sensitivity and specificity for separating 8 week from 48 hour data was $76 \%$ and $83 \%$, respectively. 
Figure 5.3 illustrates how WMA size and DSI change with infarct stiffness and infarct size. Panels A and B show more detail of the data summarized in Table 5.1, confirming that DSI is unable to distinguish between acutely ischemic and infarcted regions in this data. Figure $5.3 \mathrm{C}$ shows how WMA size changes with the ischemic or infarct area size. In the model, a WMA caused by an acutely ischemic region was clearly larger than a WMA caused by an infarct of equal size. However, this pattern of larger WMAs in acutely ischemic regions compared with infarcts of matched size does not hold in the experimental data. There are more acute ischemia cases with large ischemic regions than infarcts with large infarct areas, resulting in a significant decrease in WMA size between acutely ischemic regions and infarcts when all cases are grouped together as in Figure 5.3A, but there is not a clear difference in WMA size between acutely ischemic regions and infarcts with matched ischemic or infarct area. Figure 5.3D shows that while DSI was larger in acute ischemia than in infarcts in the models in matched infarct or ischemic size, DSI actually trends towards being smaller during acute ischemia than in infarcts in cases with larger ischemic or infarct area. We therefore conclude that while DSI is moderately effective for separating WMAs caused by healed 8 week infarcts from those caused by 48 hour infarcts, DSI cannot distinguish between WMAs caused by 8 week infarcts and those caused by acute ischemia.

\subsubsection{Acute Ischemia vs. Infarcts During Stress}

Finally, we performed preliminary model testing to ask whether DSI shows promise for distinguishing between WMAs caused by a critical stenosis and those caused by an infarct during stress testing. Figure 5.4 shows Hammer projection maps of 3DFS in both models. For an infarct or ischemic region covering $32 \%$ of the LV surface, both predicted 
WMA size and predicted DSI are much larger in an infarct compared with the critical stenosis model.

\subsection{Discussion}

In this study we found that while modeling results predicted that DSI would be larger in WMAs caused by acutely ischemic regions than in those caused by infarcts, these results were only replicated between the 48 hour and 8 week time points following occlusion and not between healed 8 week infarcts and acute ischemia in experimental data. Further, overlap in DSI between the 48 hour and 8 week groups decreased the sensitivity and specificity for separating infarcts from acutely ischemic regions. While there are some important differences between the models and experiments and in the experimental time points which may have influenced this data, these results suggest that DSI may not be effective for distinguishing between WMAs caused by infarcts and those caused by acute ischemia during rest. In initial FE modeling of peak stress, DSI detected more bulging in pre-existing infarcts than in regions of demand ischemia. For application

during stress testing, DSI may be effective for separating infarcts from demand ischemia by detecting more bulging in infarcts.

\subsubsection{Acute Ischemia vs. Infarcts At Rest}

The FE model of acute ischemia and infarcts at rest confirmed previous modeling results in which DSI was smaller in WMAs caused by healed infarcts than in WMAs caused by acutely ischemic regions, regardless of the size of the ischemic or infarcted region. These results were further confirmed in dogs with infarcts measured 8 weeks after 
occlusion compared with infarcts measured 48 hours after occlusion, although the overlap between groups decreased the sensitivity and specificity of using DSI to separate the two groups. However, DSI was not different between dogs with acutely ischemic regions and the 8-week-old infarct group. In fact, we observed more bulging, as measured by DSI, in infarcts than during acute ischemia. The experimental data were from studies originally designed for other purposes, and there are some clear differences in experimental methods. What could cause this difference between the experiments and the model?

The use of different imaging modalities may have had an effect on the 3DFS results. A previous study in our lab compared 3DFS measured from 3DE and CMR in healthy volunteers, and found larger values on average measured in CMR than 3DE. ${ }^{80}$ This difference is likely caused by the difficulty in distinguishing between trabeculations and endocardium in 3DE compared with CMR, which often causes the cavity volume to be measured as larger in CMR than $3 D E .^{92}$ It is possible, then, that less bulging was measured in 3DE because trabeculations masked some of the bulging, while these trabeculations were included in the cavity in CMR data. We already saw that trabeculations likely increase 3DFS measured from 3DE during peak stress, as 3DFS in dogs during dobutamine stress was much higher than 3DFS in the FE model of peak stress in Chapter 3, even though strains were similar between the model and data. While similar differences between experiments and the model were not observed at rest, if trabeculations cause inward endocardial motion to appear greater than it actually is during peak stress, they may influence 3DFS in WMAs as well. A comparison of 3DFS measured by both 3DE and CMR in subjects with WMAs would be necessary to test if this is the case. However, even if 3DFS is significantly different when measured from 
different imaging modalities, abnormalities should still exhibit expected trends within the same modality. The expected trends were observed in infarcts imaged with CMR 48 hours and 8 weeks post-occlusion. Therefore, we speculate that we did not observe the same trends in DSI between the model and acutely ischemic regions and infarcts in vivo because the infarcts in this study were imaged with CMR, while acutely ischemic regions were imaged with 3DE. In further work, we plan to acquire 3DE images of dogs with healed infarcts to test DSI without possible influence of different imaging modalities.

It is also possible that typical in vivo variation in 3DFS may also have obscured any expected trends in DSI, instead of differences in imaging modality. This variation is the main reason that $\triangle 3 D F S$ was more effective than 3DFS for detecting a critical stenosis, as explained in Chapter 4. Perhaps measuring bulging as the difference from average resting 3DFS, or DSI of $\triangle 3 \mathrm{DFS}$, would be more effective? To test this, $\triangle 3 \mathrm{DFS}$ in the acute and 8 week data was measured and DSI was calculated with the cutoff of $\Delta 3 \mathrm{DFS}=-10 \%$, a cutoff which was previously used to detect acutely ischemic regions. ${ }^{77}$ In this case, DSI equaled $0.15 \pm 0.02$ in acutely ischemic regions and $0.19 \pm 0.07$ in infarcts $(p=0.026)$. A significant difference was observed, but overlap was high and bulging was measured to be higher in infarcts than acutely ischemic regions, directly contradicting our modeling results. While it's possible that differences in DSI were obscured by experimental variability in vivo, the change in DSI from acutely ischemic regions to healed infarcts in dogs does not match model predictions even when normalized by average resting 3DFS. 


\subsubsection{Acute Ischemia vs. Infarcts During Stress}

While DSI was originally tested for detecting infarcts during resting conditions, the most useful possible application of DSI would be during stress testing. Resting WMAs are rarely caused by acute ischemia, but a stress-induced WMA may be caused by either an infarct or a flow-limiting stenosis. This is a more stringent test of DSI because while LV pressures are greater during stress so that bulging is likely more severe, a region of demand ischemia still exerts some force and is therefore stiffer than a resting acutely ischemic region with no force generation. This modeling study asks, in effect, if the stiffness of a region of demand ischemia can be considered stiffer than a healed infarct with no force generation. We observed more bulging and higher DSI in the infarct model than the critical stenosis model, where force generation in the ischemic region was half of the baseline force generation. This suggests that the infarct is less stiff than a slightly contracting ischemic region.

To verify this conclusion, the stiffness of the infarct passive material model was compared to partially-activated myocardium with normal passive stiffness by performing a simple uniaxial stretch of a cube in the FE model with aligned fibers. The cube with the infarct passive material properties was stretched in the fiber direction, and resultant stress is displayed as a function of stretch from the undeformed configuration in Figure 5.5. The cube with normal passive material properties was stretched, peak active stress generation was applied with the demand ischemia active properties $\left(\sigma_{\mathrm{act}}=25 \mathrm{kPa}\right)$, and resultant stress is displayed in Figure 5.5. The stiffness of the demand ischemia cube is greater than the infarct when stretch is below 1.15. Fiber stretch with respect to the undeformed configuration at ES was 0.94 in demand ischemia and 1.02 in infarcts during peak stress in these models. At those values of stretch, a region of demand 
ischemia is significantly stiffer than an infarct with stiff passive properties. Further, because this model is of an especially severe flow reduction, critical stenoses observed in vivo are likely even stiffer and therefore bulge even less. These results indicate that DSI may be effective for detecting WMAs caused by infarcts, just in an unexpected way by detecting regions which bulge more than WMAs caused by acute demand ischemia. However, the predicted difference in DSI between regions of demand ischemia and infarcts is similar to the difference predicted by the model between acute ischemia and infarcts during rest. The experiments showed enough variability in DSI that this difference was not large enough to distinguish between infarcts and acute ischemia at rest. DSI must be evaluated in vivo in regions of demand ischemia and infarcts at stress to test whether variation is low enough for DSI to be effective.

\subsection{Conclusions}

FE modeling results support the hypothesis that DSI may be useful for distinguishing between WMAs caused by infarcts and those caused by acute ischemia. The experimental data in this study only supports this hypothesis for distinguishing between infarcts imaged 48 hours and 8 weeks post-occlusion, and not for distinguishing between 8 week infarcts and acute ischemia. Even when model results were confirmed in experimental data between 48 hour and 8 week infarcts, overlap between groups decreased the sensitivity and specificity of DSI for detecting healed infarcts. The use of different imaging modalities at different time points makes it difficult to draw conclusions from the acutely ischemic experimental data. In addition, FE modeling suggests that DSI may be useful for distinguishing between regions of demand ischemia and infarcts during stress testing by detecting decreased bulging in demand ischemia compared with 
infarcts. These results must be tested experimentally in similar experimental conditions with one imaging modality before drawing conclusions about the clinical utility of DSI. 
Table 5.1 Average experimental data for each timepoint

\begin{tabular}{|c|c|c|c|c|c|c|c|c|c|}
\hline & \multicolumn{3}{|c|}{ Acute } & \multicolumn{3}{|c|}{48 Hour } & \multicolumn{3}{|c|}{8 Week } \\
\hline Ischemia/Infarct Size (\% LV) & 26 & \pm & 14 & 36 & \pm & 11 & 16 & \pm & $11^{* \ddagger}$ \\
\hline WMA Size (\% LV) & 14 & \pm & 10 & 29 & \pm & $10^{*}$ & 7 & \pm & 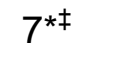 \\
\hline DSI & 0.049 & \pm & 0.069 & 0.079 & \pm & 0.037 & 0.035 & \pm & $0.027^{\ddagger}$ \\
\hline
\end{tabular}

${ }^{*} \mathrm{p}<0.05$ vs. Acute

${ }^{\ddagger} p<0.05$ vs. 48 Hour 


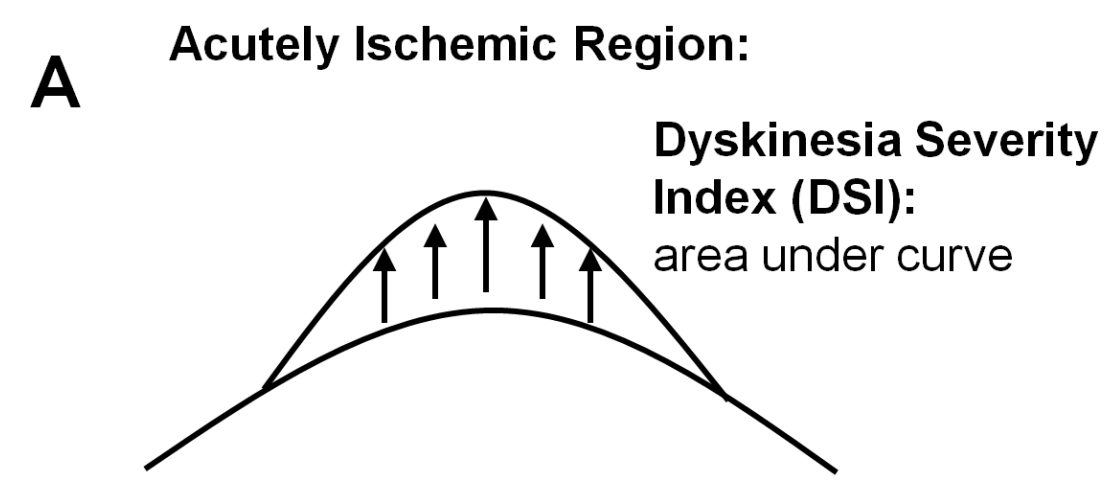

\section{Existing Infarct:}

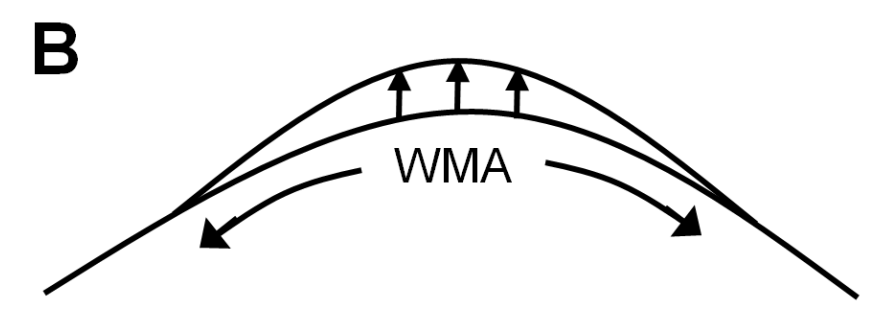

Figure 5.1 Illustration of dyskinesia severity

2D short axis views of the ischemic LV $(\mathbf{A})$ and infarcted LV $(\mathbf{B})$ show the difference in severity of bulging. This severity is measured by the 3D equivalent of the area under the curve, normalized by the WMA area. 


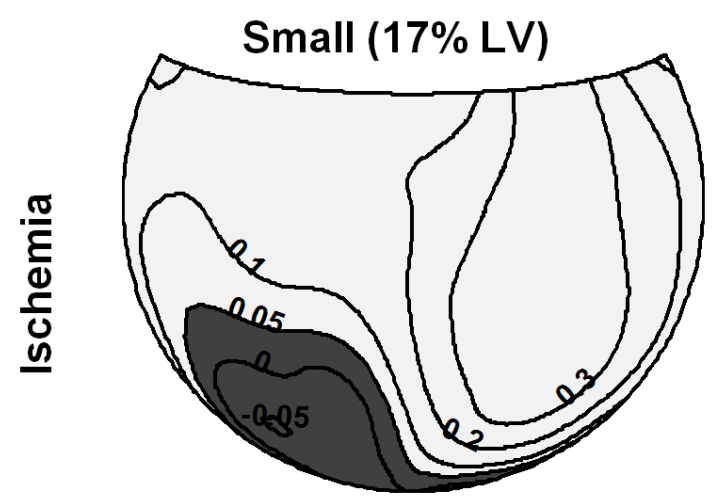

3 DFS $<0.05$ Size $=14 \%$

$\mathrm{DSI}=0.050$

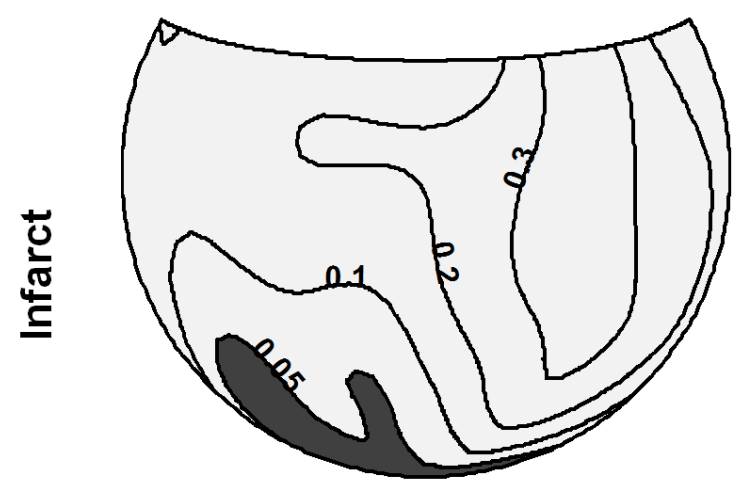

$3 D F S<0.05$ Size $=5 \%$ $\mathrm{DSI}=0.011$

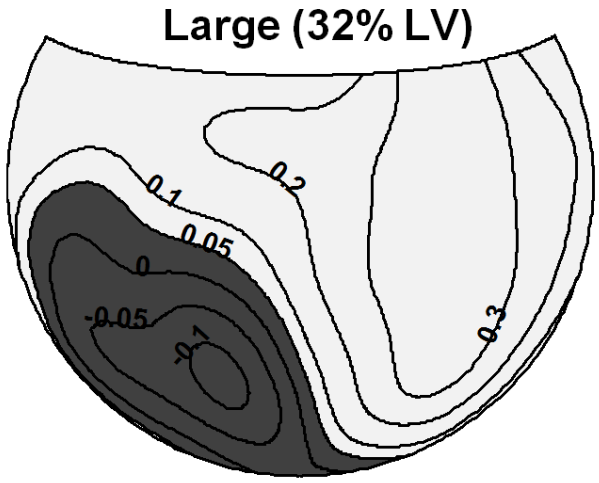

3 DFS $<0.05$ Size $=26 \%$

$\mathrm{DSI}=0.073$

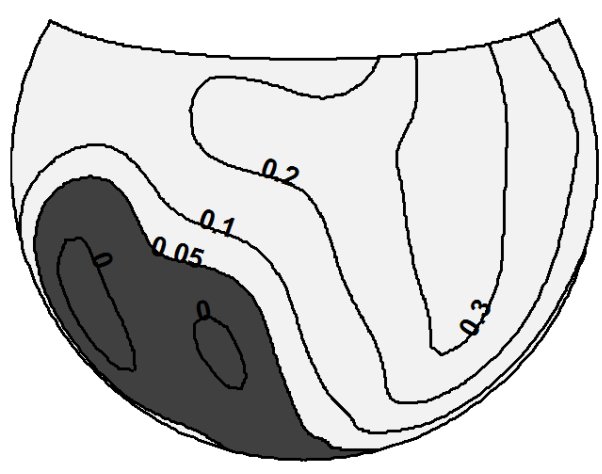

3DFS $<0.05$ Size $=18 \%$

$\mathrm{DSI}=0.029$

Figure 5.2 Hammer maps of 3DFS in FE models of acutely ischemic regions and healed infarcts

Hammer maps of 3DFS show an anterior apical WMA in Small (left column) and Large (right column) regions of acute ischemia (top row) or infarcts (bottom row). 

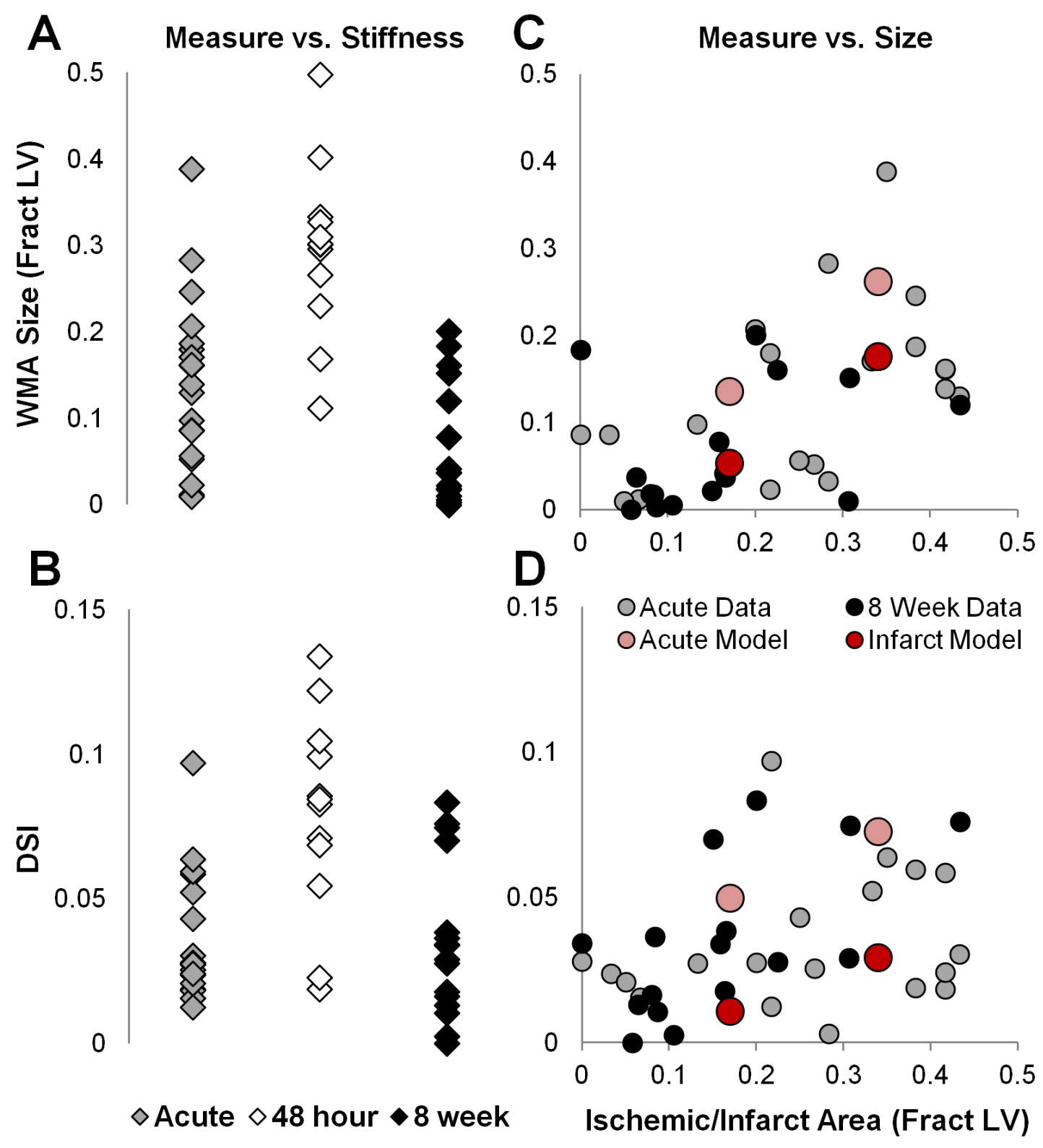

Figure 5.3 WMA size and DSI in canines during acute ischemia, 48 hours, and 8 weeks post-occlusion

See caption on following page. 
Figure 5.3 Stiff infarcts cause smaller WMAs on average than acute ischemia or 48 hour infarcts, as expected (A). However, in contrast to model results, DSI does not decrease between acute ischemia and 8 week infarcts, and actually increases for 48 hour infarcts (B). On closer inspection, we see that WMA size does not appear to be larger in acutely ischemic regions (grey) compared with infarcts (black) of the same size, which is in contrast to modeling results in red and pink (C). Finally, while DSI in the models was much larger in acutely ischemic regions than in infarcts, DSI in the data actually trends towards being smaller in acutely ischemic regions compared with infarcts of the same size (D). 

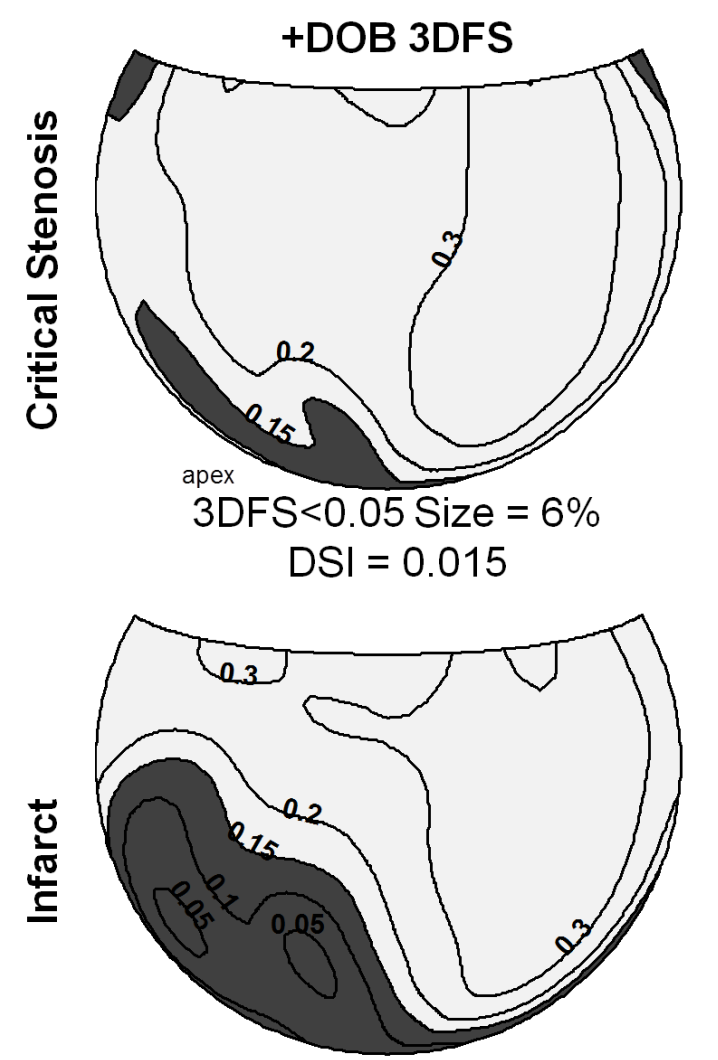

3 DFS $<0.05$ Size $=23 \%$
DSI $=0.056$

Figure 5.4 3DFS during peak stress in infarcts and demand ischemia

Hammer projection maps of 3DFS during peak stress (+DOB) show a smaller anterior apical WMA and smaller DSI in a model of a severe critical stenosis (top) than in an infarct model (bottom). 


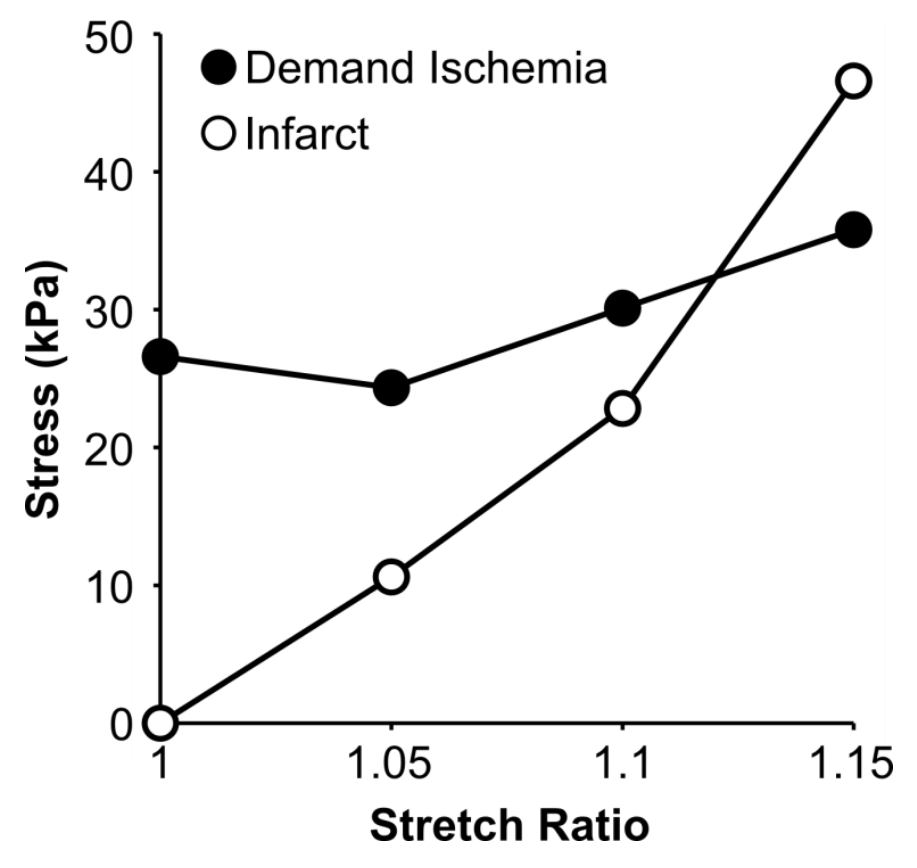

Figure 5.5 Myocardial stiffness in infarcts and demand ischemia

Stress was measured as a function of stretch in cube model of infarcted passive myocardium and partially-activated myocardium such as in a region of demand ischemia. 


\section{Wall Motion in Low-Risk Patients Undergoing Dobutamine Stress Echocardiography}

\subsection{Introduction}

Quantitative wall motion analysis using three-dimensional fractional shortening (3DFS) was effective for detecting critical stenoses during dobutamine stress echocardiography (DSE) in dogs, as detailed in Chapter 4. Therefore, 3DFS may be useful for improving diagnosis of wall motion abnormalities (WMAs) clinically. This wall motion analysis had the best diagnostic efficacy for detecting a critical stenosis when using $\triangle 3 D F S$, defined as 3DFS minus the average 3DFS at each location on the left ventricle (LV) during peak stress in dogs with no stenosis. The clinical diagnostic utility of $\triangle 3 D F S$ will depend on establishing the average 3DFS at peak stress in patients without coronary artery disease $(C A D)$, and whether the amount of variability observed is low enough for effective normalization. Therefore, function in patients without CAD during both rest and stress must be established.

Current guidelines state that normally-perfused regions of the LV are expected to increase in function and velocity of contraction at stress compared with rest, whereas regions with a decrease in function or velocity of contraction at stress compared with low dose are considered WMAs. ${ }^{23}$ Consistent with that expectation, ejection fraction (EF), which is often assessed qualitatively during routine review of echocardiograms, is assumed to increase with stress in normally-functioning hearts. However, the data reported in the literature presents a more uncertain view. Data describing global function during dobutamine stress is quite limited, with one study reporting increased $\mathrm{EF}^{131}$ and a 
few reporting either no change in stroke volume (SV) at intermediate stress of 15-20 $\mu \mathrm{g} / \mathrm{kg} / \mathrm{min}^{132}$ or increased SV at intermediate stress but no change in SV at peak stress. ${ }^{133,134}$ Data describing the regional mechanical function of the left ventricle (LV) are more prevalent for established measures of regional mechanics, but can also be conflicting. Fractional shortening measured from two-dimensional echocardiography was reported to increase at intermediate stress ${ }^{131}$ and not change at peak stress ${ }^{135}$ compared with rest. In a clinical population, longitudinal and circumferential strain have been reported to both increase in magnitude ${ }^{93}$ and not change ${ }^{67,94}$ with stress, while strain rate magnitude has been reported to increase with stress. ${ }^{66,67,94}$

EF, fractional shortening, and strain are normalized by diastolic values, and can be confusing to interpret when these diastolic values change. Therefore, it is important to understand both un-normalized volumetric changes during stress and regional function changes to detect abnormal function. In this study, we collected three-dimensional echocardiography (3DE) images from patients who underwent routine DSE at the University of Virginia echocardiography lab but were at low risk for CAD, and analyzed the images to determine the normal changes in volumes, EF, and 3DFS that occur during stress testing. Evaluating the change between rest and stress and variation in these measures is the first step toward using these measures to distinguish between normal and abnormal mechanical behavior during stress testing. 


\subsection{Methods}

\subsubsection{Study Population}

The protocol for this study was approved by the University of Virginia Internal Review Board for Health Sciences Research. Data were collected from 193 patients who were already referred to receive a standard dobutamine stress echocardiography exam at the University of Virginia Health System from 2009 to 2011. Stress was induced with a graded dobutamine infusion starting at $5 \mu \mathrm{g} / \mathrm{kg} / \mathrm{min}$ and increasing at 3-minute intervals to $10,20,30$, and $40 \mu \mathrm{g} / \mathrm{kg} / \mathrm{min}$, and standard two-dimensional (2D) imaging was performed. In addition to the standard exam, 3DE images were acquired during rest and peak stress by an experienced sonographer using a Philips iE33 ultrasound system and either an x3-1 or x5-1 matrix array transducer (Philips Medical Systems, Andover, MA). Data was exported to Tomtec 4D LV-Analysis ${ }^{\circledR}$ software version 2.6 (Tomtec Imaging Systems, Unterschleissheim, Germany) for evaluation of image quality and wall motion analysis. The patient was included in the study if the endocardial border was visible over most of the LV surface in at least one 3DE image at both rest and peak stress, and if each image had sufficient frame rate to capture more than five frames per beat. Studies using contrast were excluded due to difficulties in 3DE acquisition. Patients were classified as either low, intermediate, or high risk for CAD based on an established risk factor scoring system. ${ }^{136}$ In the original published validation of this scoring system, $16 \%$ of patients defined as low risk had at least one coronary vessel that displayed $>50 \%$ narrowing by coronary angiography, and $8 \%$ had at least two vessels with $>70 \%$ narrowing. One patient with a positive stress test by standard clinical diagnosis and subsequent catheterization was analyzed as an example; otherwise, those with 
intermediate or high risk for CAD, as well as a history of any cardiac myopathy such as myocardial infarction, were excluded from the analysis presented here.

\subsubsection{Data Analysis}

Images were segmented and 3DFS was measured using methods detailed in Chapter 3. Volumes and EF were measured as the volume enclosed by the fitted surface. To compare these quantitative results to standard methods of diagnosis, EF was visually estimated from 2D images in $5 \%$ increments by two blinded, expert cardiologists in a subset of 15 patients. The average and standard deviation of 3DFS for all patients was calculated by averaging the 3DFS value at each $(\mu, \theta)$ location on the LV surface, and $\triangle$ SDFS was calculated by subtracting this average value from stress 3DFS in individual patients. $\triangle 3$ DFS analysis was deemed positive for ischemia if greater than $5 \%$ of the LV surface was below a given $\triangle$ 3DFS threshold. Average 3DFS in each segment of the standard cardiac 16 -segment model ${ }^{137}$ was calculated in each patient at rest and stress to detect regions of high variance. Significance of changes between rest and stress were computed using a two-tailed, paired student's t-test.

\subsection{Results}

Of the 193 studies collected, 92 had acceptable image quality. Of these, 20 studies met all inclusion criteria and were analyzed. The remaining 72 patients had either intermediate or high risk of CAD, or had a positive stress echo study. This cohort had a mean age of $53 \pm 12$ years, with seven men, nine Caucasians, 10 African Americans, 
one Latino, and four diabetics. Each patient's dobutamine stress echo was interpreted as negative for CAD.

Figure 6.1 shows LV volumes measured from 3DE for rest and peak stress. Enddiastolic volumes (EDV), end-systolic volumes (ESV), and SV at stress were significantly smaller than at rest. However, because EDV and ESV decreased proportionately, EF was not different between rest and peak stress. In a subset of 15 patients, while quantitatively-measured EF was not different between rest and peak stress, EF was visually interpreted to increase from rest to peak stress by two blinded, expert cardiologists (Figure 6.2).

Average 3DFS for rest and stress is displayed on Hammer maps in Figures 6.3A and B. These data show the same pattern of 3DFS increasing from the base to the apex that was observed in dogs in Chapter 5. 3DFS averaged over all patients and locations on the LV surface was $0.20 \pm 0.05$ at rest and $0.21 \pm 0.07$ at stress $(p=0.16$, no significant difference). Hammer maps showing the standard deviation of 3DFS for each location on the LV surface are shown in Figures 6.3C and D. Variation is larger at stress (average 0.10) than at rest (average 0.08), with the highest variation occurring at the apex and lateral wall. Differences between 3DFS in rest and stress were only significant in the basal anterolateral and apical septal segments of a standard 16-segment model (Figure 6.4). $\triangle 3$ DFS was calculated for each individual patient to determine the number of false wall motion abnormalities that would be detected in this population. Using the threshold of $\triangle 3$ DFS $<-0.275$, which was the optimal threshold for detecting a critical stenosis in Chapter 4, 95\% of patients were correctly diagnosed as negative for a wall motion abnormality. Specificity was $90 \%$ for a threshold of $\triangle 3$ DFS $<-0.2$, and $75 \%$ for $\triangle 3 D F S<-0.1$. 
While the main objective of this study was to evaluate 3DFS in a normal patient population, one patient with CAD and a positive stress test was analyzed as an example of how $\triangle 3 D F S$ may be used clinically. Visual analysis of the 2D ultrasound images acquired during this dobutamine stress test indicated an inducible wall motion abnormality on the anteroseptal and inferoseptal wall. Coronary angiography, completed five months after the stress test, detected a coronary stenosis in the left main artery with a $50 \%$ reduction in arterial diameter and fractional flow reserve of 0.885 . This stenosis was categorized as subcritical both angiographically $\left(>70 \%\right.$ diameter reduction cutoff ${ }^{8}$ ) and functionally $\left(<0.8\right.$ fractional flow reserve cutoff $\left.{ }^{10}\right)$. Both 3DFS and $\triangle 3 D F S$ measured at peak stress are displayed in Figure 6.5. A region of abnormal wall motion is not apparent from the 3DFS map (panel A), but a region of reduced $\triangle 3$ DFS is apparent on the septum near the apex (panel B), which is within the perfusion bed of the left main artery. However, the size of the region of $\triangle 3 D F S<-0.2$, one possible cutoff, is only $4.9 \%$ of the LV, and there is no abnormal wall motion with a cutoff of $\triangle 3 D F S<-0.275$. This borderline-insignificant stenosis by angiography produced a borderline-insignificant wall motion abnormality by 3DFS analysis.

\subsection{Discussion}

In this study, we found that while volumes indeed decrease with stress, EF and 3DFS do not change significantly. In contrast, two expert cardiologists visually interpreted the images to have an increased EF at stress. 3DFS in patients displays the same regional patterns as were measured in canines, albeit with smaller 3DFS values. The variability in 3DFS response is higher at stress, especially near the apex, and 3DFS at the apex 
increases slightly during stress. However, this variability is low enough to allow few false positive diagnoses in this data set.

\subsubsection{Perception vs. Reality in DSE}

While our results show decreasing volumes and no change in EF from rest to stress, these results for global volumetric behavior are contrary to current guidelines and common perceptions among cardiologists. Cardiologists are commonly trained to visually estimate EF in increments of $5 \%$ based on LV size, wall motion, wall thickening, and contractility. This visual estimation method has been previously validated with volumes measured from $3 \mathrm{DE},{ }^{138}$ and was fairly accurate at rest for both experts in this study. While visual estimation of EF during stress is less common and can be expected to be less accurate, it provides a measure of the perceived change in function during stress. When viewing echocardiograms during stress, function appears to increase because the ESV is significantly smaller at stress and because the inward motion of the wall appears faster due to the increased heart rate. However, these increases in function are offset by the decreased EDV, as filling time has decreased, and as a result the EF does not actually change. Multiple studies of healthy individuals in literature are conflicting on the pattern of EF or SV during mid-dose and high-dose dobutamine stress, ${ }^{131-133}$ yet the perception of increased EF during stress is reported to be wellestablished in the latest clinical guidelines for interpreting stress echocardiograms. ${ }^{23} \mathrm{We}$ found this difference between measurements and perception on a smaller scale in this study, in the difference between the quantitatively-measured and visually-estimated EF.

These results emphasize the importance of quantitative measurement in establishing diagnostic criteria and in performing diagnoses with imaging. This is 
especially true in evaluating regional cardiac function. Similar to global function, current guidelines state that an increase in regional function and velocity of contraction is expected in normal individuals. Since regional wall motion is usually assessed visually or by "semi-quantitatively" categorizing regions by assigning scores based on visual grading, this perception persists. In literature, most studies that evaluate wall motion during DSE have been based on analysis of wall motion scores which are assigned by visual analysis. Some of these studies reported increased wall motion scores at stress, ${ }^{139}$ while others reported no change in wall motion from rest to peak stress. ${ }^{140}$ The current study confirms the results of a previous study of quantitatively-measured LV dimensions and fractional shortening from 2D ultrasound, ${ }^{135}$ in which decreased ED dimensions resulted in no increase of EF or fractional shortening from rest to stress. These results were surprising in 1995, but have yet to be accepted into clinical practice.

Even though the common perception of cardiac function during stress does not match quantitative measurements, stress echocardiography using visual analysis is still a fairly sensitive and specific test for detecting WMAs caused by CAD. This seems to indicate that there are real differences between normal and abnormal function, but perhaps the changes that are seen by eye are not well-articulated in the current guidelines. Establishing quantitative measurements of function in cases of normal and abnormal function could not only improve repeatability and accuracy of diagnosis, but also provide better ways for describing the behavior of a WMA in both research studies and in training new cardiologists. Overall, these observations confirm the fact that what we see by eye can be deceiving, and that a move to using quantitative diagnosis for routine clinical use during DSE and imaging studies in general should be of high priority for the practicing clinician. 


\subsubsection{Application of 3DFS to Clinical Cases}

For 3DFS to effectively detect WMAs during stress testing, it must clearly distinguish between normal and abnormal regional function at peak stress. Variation in normal function must be small enough among patients so that WMAs are distinguishable in more than the most severe abnormalities. We found an average 3DFS of 0.21 and standard deviation of 0.07 at stress, with higher values and variation near the apex and lower values and variation near the base. Using the optimal $\triangle 3 D F S$ cutoff for detecting a critical stenosis in dogs, only one false positive wall motion abnormality was observed in this data set. This low variability bodes well for the application of 3DFS to the detection of WMAs during clinical stress testing.

Spatial variation of regional function can also hinder the effectiveness of quantitative measures. Strain has previously been shown to vary over the surface of the LV during rest, with smaller magnitudes of circumferential and longitudinal strain near the base and larger magnitudes near the apex. ${ }^{141}$ Both here and in Chapter 4 , we see similar variation in 3DFS. Tethering to the mitral valve limits wall motion near the base, and inward movement is greatest at the apex due to its distance from the valves. A region of decreased 3DFS is present on the basal posterolateral wall due to the presence of the tricuspid valve, which is cut off during segmentation. These regional heterogeneities are consistent and therefore can be taken into account when using 3DFS to detect WMAs.

In Chapter 4, we subtracted the average control stress 3DFS in dogs from individual studies to effectively detect critical stenoses. The use of a similar analysis in patients to detect WMAs appears promising due to the similar 3DFS patterns and variation measured in patients. We plan to use the average stress 3DFS measured in 
this study to apply similar 3DFS normalization in patients, and then test the ability of 3DFS to detect CAD in patients undergoing DSE. A larger study of $\triangle 3 D F S$ in patients who later undergo angiography is needed to establish a clinical $\triangle 3 D F S$ threshold that is both sensitive and specific to the presence of significant CAD, and to evaluate the effectiveness of $\triangle 3$ DFS for detecting wall motion abnormalities compared with other methods. An example of this analysis in a patient who received angiography is shown in Figure 6.5. This patient had visually-diagnosed wall motion abnormalities by DSE, but the stenosis was deemed subcritical by angiography. $\triangle$ DDFS analysis showed a small wall motion abnormality that is close to proposed $\triangle$ 3DFS cutoffs. While the $\triangle$ 3DFS diagnosis cannot be definitively measured without an established cutoff, the fact that this wall motion abnormality is bordering on significance by $\triangle$ BDFS when it also was almost significant by angiography is promising for future clinical testing of $\triangle 3 D F S$.

\subsection{Conclusion}

In summary, LV volumes were found to decrease during dobutamine stress in patients at low risk for CAD, while EF and 3DFS did not change between rest and peak stress. Regional variation in 3DFS was predictable, and variation amongst patients was low. These results demonstrate the importance of using quantitative measurements in imaging, and show promise for application of 3DFS for detecting WMAs during clinical stress testing. 


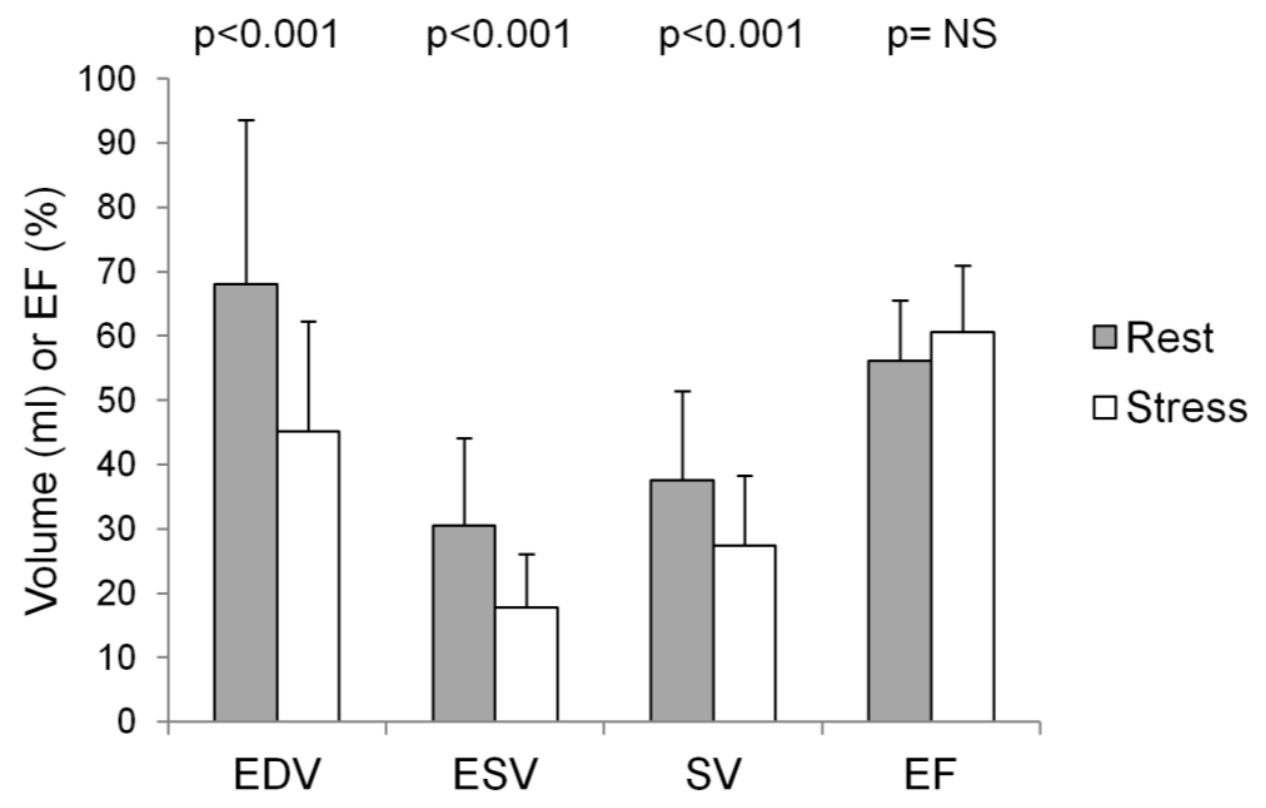

Figure 6.1 Average volumes and EF

While average LV volumes and stroke volume (SV) decrease during peak stress, EF is not different between rest and stress. 


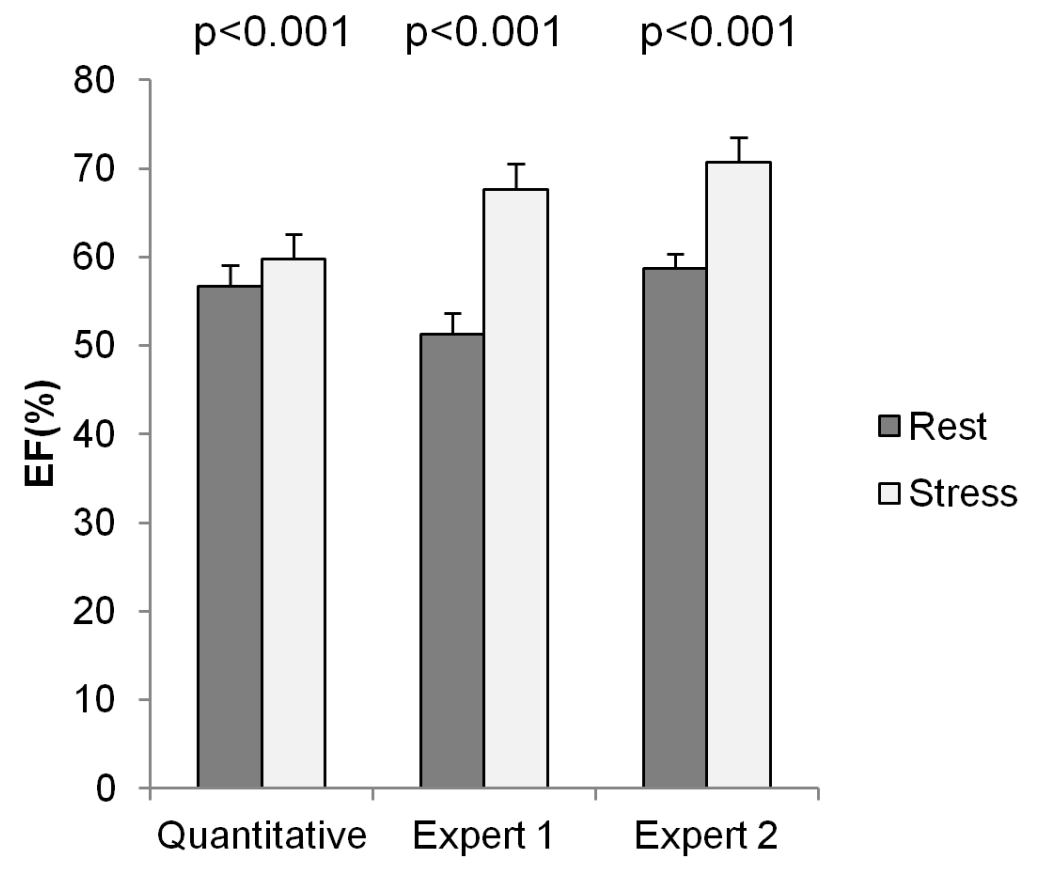

Figure 6.2 Comparison of quantitative and visually-estimated EF

In a subset of 15 patients, quantitatively-measured EF at rest and peak stress are not different. Images were visually interpreted to have increasing EF from rest to peak stress. 


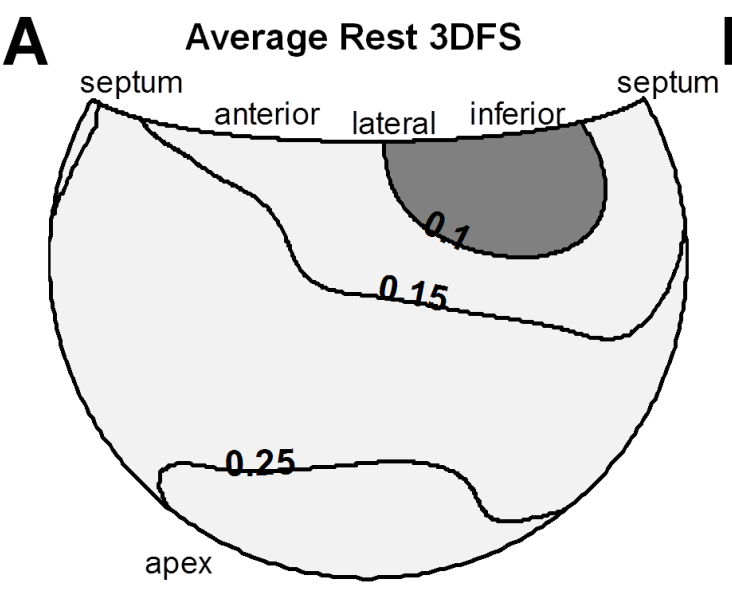

B Average Stress 3DFS

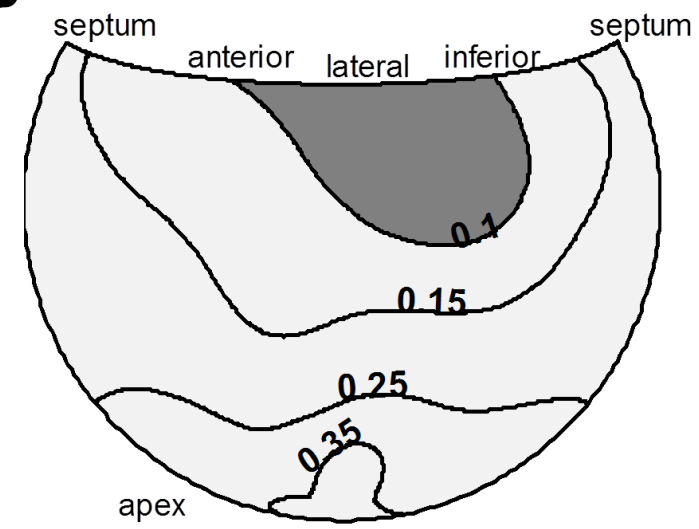

C Rest 3DFS Standard Deviation

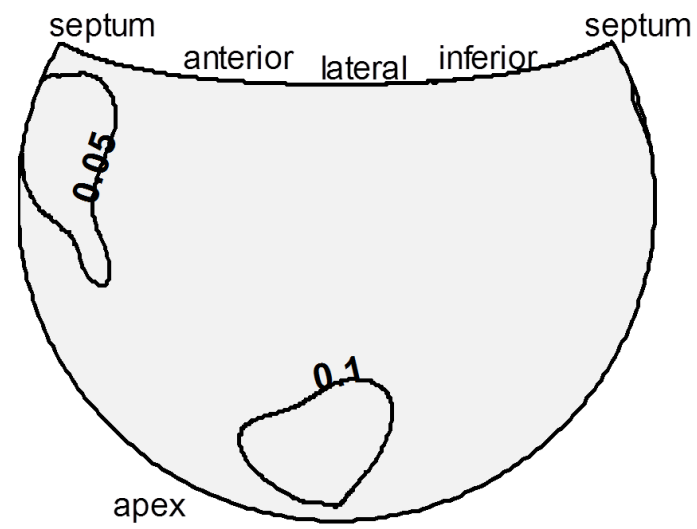

D Stress 3DFS Standard Deviation

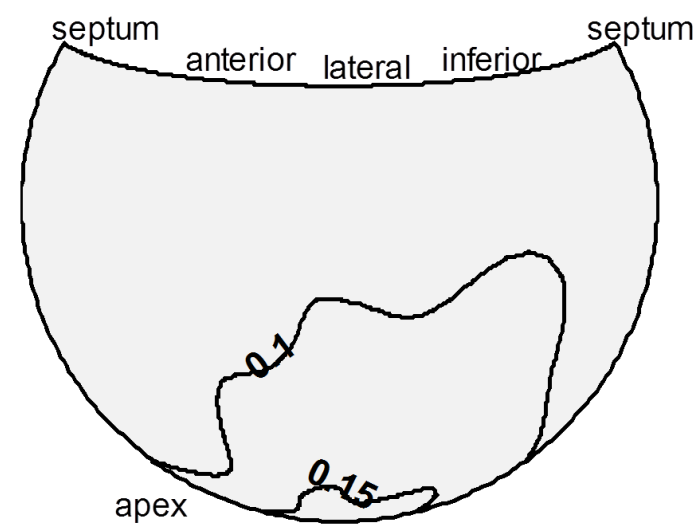

Figure 6.3 Average and standard deviation 3DFS at rest and stress

Hammer maps of 3DFS over the LV surface for rest (A) and peak dobutamine stress (B). Patterns and absolute values of 3DFS do not change significantly from rest to peak dobutamine stress. Standard deviation of 3DFS measurements in each location is slightly lower in rest (C) than in stress (D), and tends to be higher near the apex. 


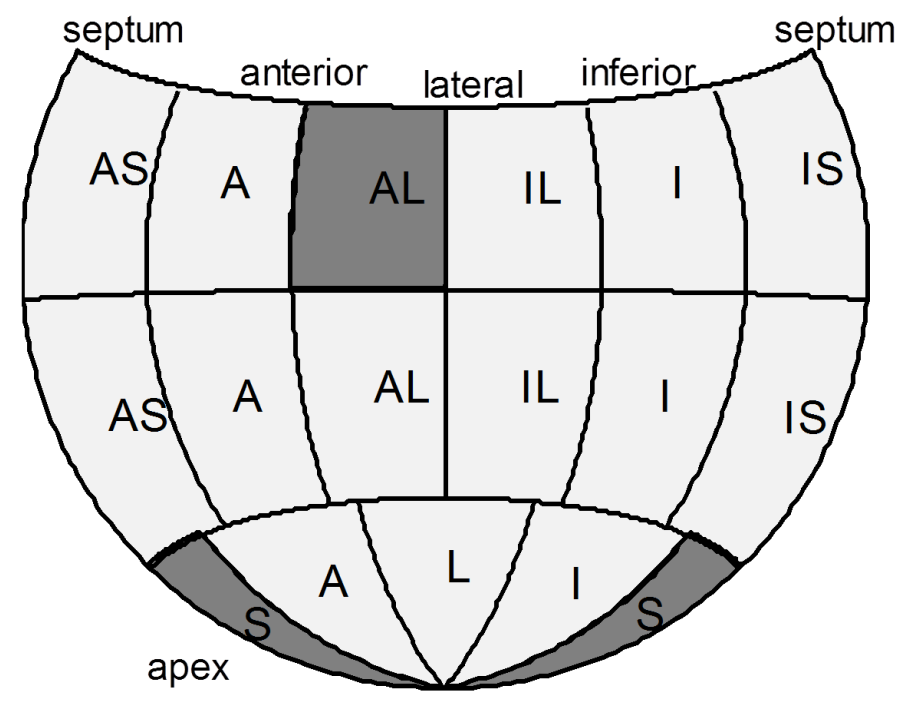

Figure 6.4 Regions of significantly different 3DFS between rest and stress

The divisions of a standard 16-segment model are shown on a Hammer projection map. Segments that displayed significantly different 3DFS between rest and stress are shaded in gray. $\mathrm{AS}=$ anteroseptal, $\mathrm{A}=$ anterior, $\mathrm{AL}=$ anterolateral, $\mathrm{IL}=$ inferolateral, $\mathrm{I}=$ inferior, IS = inferoseptal, $S=$ septal, $A=$ anterior, $L=$ lateral, I = inferior. 

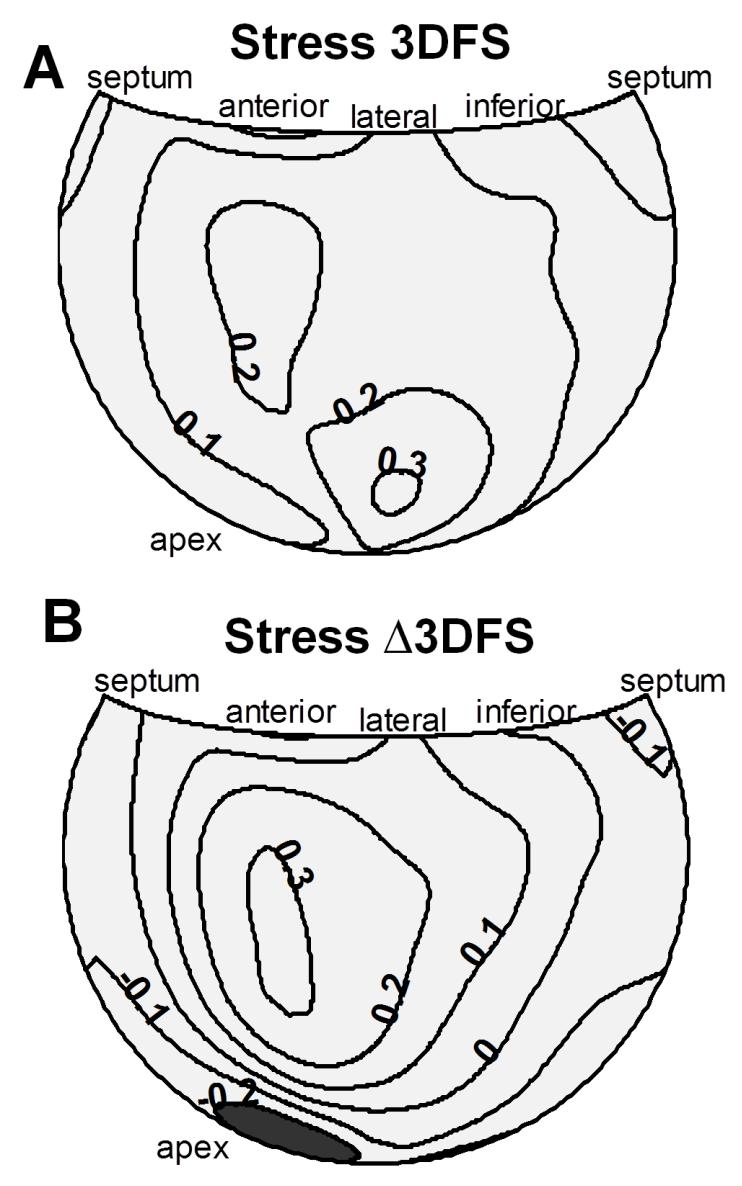

Figure 6.5 Example 3DFS analysis in one patient with CAD

3DFS analysis in one patient with a positive stress test and $50 \%$ stenosis of the left main coronary artery. An abnormality is not apparent in a map of 3DFS measured at peak stress $(\mathbf{A})$, but a region of negative $\triangle$ BDFS reveals an apical wall motion abnormality $(\mathbf{B})$. 


\section{Applications of Cardiac Mapping: Relating Lead and Infarct Location to Evaluate Cardiac Resynchronization Therapy Nonresponse}

\subsection{Introduction}

Although it improves overall survival in appropriate selected populations, ${ }^{142,143}$ implementation of cardiac resynchronization therapy (CRT) continues to be limited by nonresponse rates on the order of $30-40 \% .{ }^{144}$ These response rates may be influenced by left ventricular (LV) pacing site. Although pacing within a certain "sweet spot" in the posterolateral LV was shown to produce the optimal hemodynamic improvement in an animal model, ${ }^{145}$ clinical studies have shown significant variability in the left ventricular pacing site that results in optimal hemodynamics in human heart failure. ${ }^{146-148}$ These differences in optimal LV pacing site from patient to patient are associated with varying distributions of left ventricular electromechanical activation and myocardial scar. ${ }^{149-152}$

In the current era of surgical epicardial lead placement, quadripolar LV leads, and other technologies, it is increasingly possible to access a target LV pacing site identified by findings on cardiac imaging, even in patients with limited coronary sinus branches. The challenges are defining this target LV pacing site based on imaging data, and also determining when CRT nonresponse is due to failure to achieve this desired lead position. In this regard, an accurate and practical method for using two-dimensional (2D) fluoroscopic data from the procedure to derive three-dimensional (3D) lead position relative to pre-CRT cardiac imaging would be very useful. A number of recentlypublished studies have demonstrated methods for coregistering pre-study anatomic 
images with live fluoroscopic images to guide lead placement during implantation. ${ }^{153,154}$ These methods can measure lead location on anatomic images to within 2 to $5 \mathrm{~mm}$, but require specialized hardware and 3D fluoroscopic navigation software which add time, cost, and complexity to the procedure and are available only at tertiary academic centers. Conversely, most research studies that report lead placement for evaluation of nonresponders after implantation use qualitative methods, such as review of postoperative chest radiography after CRT, ${ }^{155}$ which suffer from limited accuracy. In this study we propose a practical and accurate method for merging LV lead position from 2D fluoroscopic images with 3D CMR anatomical data which requires only a simple calibration of the fluoroscopy imaging equipment and manual selection of easilyidentified cardiac landmarks. This calibration must only be performed once for a set of fluoroscopy equipment, and accounts for changing magnification due to changing source-to-image distance (SID) and table height. This simple algorithm allows measurement of LV lead location on anatomic images with excellent accuracy, allowing researchers to better evaluate the effects of LV anatomy, scar, function, and lead location in CRT nonresponders without requiring specialized registration equipment.

\subsection{Methods}

We propose a straightforward method, diagrammed in Figure 7.1, for registering fluoroscopic images of an implanted LV lead with standard CMR cine images which requires only calibration of the fluoroscopy equipment and careful imaging of the final lead position. Both 2D fluoroscopic imaging and 3D CMR imaging was performed (Figure 7.1, columns 2 and 3), and common anatomical landmarks are selected from each image. 3D positions were reconstructed from 2D fluoroscopic projections using 
pre-study calibration data (Figure 7.1, column 1). Finally, the landmarks were used to merge the fluoroscopic and CMR data. This process allows the lead position selected in the fluoroscopic images to be transformed into the same coordinate system as anatomical data from CMR images.

\subsubsection{Fluoroscopy Calibration}

Determining the 3D coordinates of an object viewed in a 2D fluoroscopic image requires two steps: 1) Compute the relationship between the 2D and 3D images by imaging a phantom with known geometry at every imaging angle of interest, and 2) Measure and account for the change in the 2D fluoroscopic image that occurs with changes in camera magnification. If this information is known, the 3D coordinates of any object can be reconstructed from two images acquired at any calibrated view angle and camera magnification setting.

\section{Fluoroscopy 3D Reconstruction}

Fluoroscopy equipment was calibrated by imaging a cylindrical Plexiglass phantom embedded with 17 lead beads of radius $0.5 \mathrm{~mm}$ forming a spiral curve down its length. Calibration images were acquired for right and left anterior oblique angles (RAO and LAO) at 20, 22, 24, 26, 28, and 30 degrees and for the anterior-posterior (AP) view, which represent the range of possible standard clinical views, without moving the table and at a standard table height and SID (Figure 7.2A, B). The coordinates for each bead were manually selected for each image and used to calculate the relationship between these 2D coordinates and the physical 3D coordinates of the phantom beads. Mathematically, the relationship between $i$ projected 2D points and their location in a certain $3 D$ space is given by ${ }^{156}$ 


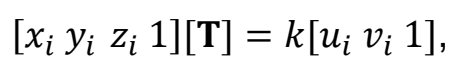

where $\mathbf{T}$ is a $4 \times 3$ transformation matrix that describes the relationship of $2 \mathrm{D}$ projected points with the coordinates $\left(u_{i}, V_{i}\right)$ and scaling factor $k$ to their coordinates in a defined 3D space $\left(x_{i}, y_{i}, z_{i}\right)$. There exists a single, unique transformation matrix for each $2 \mathrm{D}$ projection of the same $3 \mathrm{D}$ space. If the transformation matrices for various camera positions are calculated once for a fluoroscopy system, they can be applied to all images acquired by that system as long as the camera positions are known for each image.

The physical 3D locations of the beads relative to an "origin" bead were measured, allowing for the solution of $\mathbf{T}$ using Equation 1 for each camera angle. Once this calibration has been performed, 3D coordinates of any point selected from the fluoroscopic image can be calculated from at least two 2D projection images using Equation 2: 156

$$
\left[\begin{array}{lll}
s_{11}-s_{13} u_{1} & s_{21}-s_{23} u_{1} & s_{31}-s_{33} u_{1} \\
s_{12}-s_{13} v_{1} & s_{22}-s_{23} v_{1} & s_{32}-s_{33} v_{1} \\
t_{11}-t_{13} u_{2} & t_{21}-t_{23} u_{2} & t_{31}-t_{33} u_{2} \\
t_{12}-t_{13} v_{2} & t_{22}-t_{23} v_{2} & t_{32}-t_{33} v_{2}
\end{array}\right]\left[\begin{array}{l}
x \\
y \\
z
\end{array}\right]=\left[\begin{array}{l}
s_{43} u_{1}-s_{41} \\
s_{43} v_{1}-s_{42} \\
t_{43} u_{2}-t_{41} \\
t_{43} v_{2}-t_{42}
\end{array}\right]
$$

where $\left(u_{1}, v_{1}\right)$ and $\left(u_{2}, v_{2}\right)$ refer to the 2D coordinates of the same point in two different views and $s_{i j}$ and $t_{i j}$ refer to the elements of the corresponding transformation matrices. $3 \mathrm{D}$ coordinates were reconstructed by acquiring two images of the object from orthogonal views, such as the LAO20 and RAO20 images shown in Figure 7.2B, and then computing the 3D coordinates with Equation 2 using the transformation matrices for those two angles. As an example, Figure $7.2 \mathrm{C}$ shows the $3 \mathrm{D}$ reconstruction of the phantom beads from 2D fluoroscopic images of the phantom. 


\section{Fluoroscopy Magnification Adjustment}

Clinical images were often acquired with camera SID and table height different than those used to compute the calibration transformation matrices. We developed an algorithm to account for these differences by treating changes in SID or table height as changes in camera magnification. Increasing or decreasing SID moves the camera nearer or further from the objective, resulting in a standard linear magnification of the image. Increasing or decreasing table height also moves the camera nearer or further from the objective in an AP image. However, in oblique angles, changing the table height also causes the image to shift parallel to the AP image angle, or in the $\mathrm{x}$-coordinate of the fluoroscopic image. Therefore, additional calibration of this shift was included in the table height adjustment. The goal of this adjustment was to convert coordinates of an image acquired at any SID or table height into its coordinates if the image had been acquired at the same SID and table height we used to compute the transformation matrices for each imaging angle. These are referred to as the calibration SID and table height.

Images of the phantom were acquired at LAO 20, AP, and RAO 20 angles at three table heights and SIDs for magnification adjustment, including the calibration SID and table height (Figure 7.3A). We verified that the relationship between $i$ coordinates of beads at a given SID $\left(x_{i}\right.$ and $\left.y_{i}\right)$ and the coordinates at the calibration SID ( $x_{c a l}$ and $\left.y c a l_{i}\right)$ was linear, as described in Equation 3:

$$
\left[\begin{array}{ll}
x_{i} & 1 \\
y_{i} & 1
\end{array}\right][\boldsymbol{A}]\left[\begin{array}{c}
S I D \\
1
\end{array}\right]=\left[\begin{array}{l}
x^{x a l} l_{i} \\
y c a l_{i}
\end{array}\right]
$$

In other words, coordinate values for a structure imaged at the calibration SID are a linear function of both the coordinates at the imaged SID and the SID of that image (Figure 7.3B). Both the slope and the intercept of the relationship between $x_{i}$ and $x_{c a l}$ 
are linear functions of SID, no matter the angle of acquisition (Figure 7.3C). The 2x2 matrix A was calculated from images of the phantom, and then used to transform every landmark or lead position selected from fluoroscopic images before merging the information from the two views. Figure 7.3D shows the result when this transformation is performed on the phantom image to successfully reconstruct coordinates at the calibration SID. Application of this SID adjustment to the phantom images in four different imaging suites resulted in average root mean square error (RMSE) of $0.4 \pm$ $0.2 \mathrm{~mm}$.

To account for images acquired at different table heights, this process was repeated by replacing SID in Equation 3 with table height. As described above, a change in table height also causes a shift in $x_{i}$ when the image is acquired at a nonzero angle (Figure 7.3A,E). This shift varies with both table height and acquisition angle (Figure 7.3F). Therefore, the $y$-intercept of the relationship between $x_{i}$ and $x_{c a l}$ was fit as a function of both table height and acquisition angle using multiple linear regression. Application of this table height adjustment to phantom images in the four imaging suites resulted in average RMSE of $0.9 \pm 0.8 \mathrm{~mm}$.

\subsubsection{Registration of Fluoroscopic and CMR Images}

Fluoroscopic and CMR Landmark Selection

Based on the requirement of 3 landmarks to achieve accurate $3 \mathrm{D}$ registration, we identified the right ventricular apex (RVA), the ostium of the coronary sinus (CSO), and the antero-lateral segment of the mitral annulus (LMA) as three anatomical landmarks that can be consistently identified in all fluoroscopic images and in CMR image slices. 
All three landmarks and LV lead position were selected in two fluoroscopic images acquired at the end of implantation, and then reconstructed into 3D coordinates using the methods detailed above. Care was taken to not move the patient table between acquiring these final images. In CMR, the RVA was selected from the four chamber cine image, CSO from an axial cine image, and the LMA landmark was selected from the basal short axis cine image. All landmarks were selected at end-diastole.

\section{Registration Methodology}

Two different coordinate systems can easily be merged by simple translation and rotation; however, small errors in the selection of one landmark can significantly alter this transformation. Identification of these landmarks is imprecise due to many factors such as difficulty in visualizing the heart shadow in fluoroscopic images, cardiac motion, and the distance between CMR slice planes. Therefore, instead of geometric rotation we used an error minimization technique which assumes: 1) the location of the LV lead relative to the designated landmarks will be similar in both the fluoroscopy and CMR coordinate system, and 2) the lead is located on the epicardium.

The epicardium was contoured from short and long axis cine CMR slices using the software Segment v1.9 R2278 (http://segment.heiberg.se) ${ }^{89}$ and a surface was fit to the epicardial contours using methods described previously by our lab. ${ }^{78}$ Possible solutions for the LV lead position were restricted to a dense mesh (40 longitudinal points by 80 circumferential points) on this fitted epicardial surface. The most likely LV lead position was chosen as the point on the epicardial surface in which the distance between the lead position and each of the three landmarks in the CMR coordinate space $\left(d m_{i}\right)$ was most similar to the equivalent distances in the fluoroscopic space $\left(d f_{i}\right)$; that is, the 
objective function $O$ in Equation 4 was calculated for the $n=3200$ locations on the epicardial surface mesh.

$$
O(n)=\sqrt{\left(d f_{1}-d m_{1}(n)\right)^{2}+\left(d f_{2}-d m_{2}(n)\right)^{2}+\left(d f_{3}-d m_{3}(n)\right)^{2}}
$$

The point which produced the minimum value for $O$ was chosen as the LV lead position in the CMR coordinate system. The difference in the distance between landmarks selected in fluoroscopy and CMR, or $d f_{i}-d m_{i}$, was used as a check to ensure that overall landmark selection was similar between fluoroscopy and CMR.

This algorithm was implemented in custom software written for Matlab (v7.14, The Mathworks, Natick, MA). A 3D surface mesh of the epicardium was plotted along with landmark and lead positions in CMR coordinates for visualizing the LV lead position with respect to cardiac anatomy. One of the six patients had a previous myocardial infarction and significant visible scar in late gadolinium enhanced (LGE) images. The enhanced scar was segmented on short axis images using Segment, and scar transmurality was linearly interpolated over the LV surface from the short axis measurements.

\subsubsection{Validation Methodology}

We used three different methods to independently test our registration technique. First, as a proof-of-concept, we tested these methods in a single canine study in which the final lead position was determined at the time of pathologic study and the lead artifact on the CMR. In six patients who underwent clinically indicated cardiac computed tomography (CT) imaging after lead implantation, we compared the computed lead position to the lead position in the CT images. Finally, the computed lead position was 
compared to the anatomy of the implantation vein in one patient with pre-procedure CS venography $(n=1)$, in which the full course of the coronary sinus branch that eventually received the lead was visualized fully on the MR study. Post-CRT CT scans were only performed in patients with a clinical cardiac CT indication in order to minimize patient exposure to radiation and intravenous contrast.

\section{Validation Protocol -- Canine}

The canine protocol was approved by the Animal Care and Use Committee (ACUC) of the University of Virginia. The protocol, performed in one canine, required open chest surgery with appropriate sedation. A right ventricular (RV) lead was inserted in the RV apical septum via the internal jugular vein. The LV lead was implanted on the posterolateral epicardium. Radiopaque beads were sutured onto the epicardium near the RV apex and at the base near the CSO and LMA. The leads were connected to a permanent pacemaker, which was implanted in the neck. The chest and surgical sites were then closed, and the animal was allowed to recover. Post-surgical CMR imaging was then performed as described below. The animal was then taken to the fluoroscopy lab, and the pacing system was imaged in multiple fluoroscopic projections. After imaging, the animal was euthanized. The heart was explanted and fixed in formalin for post-mortem analysis. The LV was cut into short axis slices and imaged to verify the location of the LV epicardial lead. Landmarks were selected in fluoroscopic images using the radiopaque beads, and the registration error was calculated as the distance between the predicted lead position and the LV lead shadow on CMR imaging, with histologic confirmation of this lead location, as described above. 


\section{Validation Protocol -- Human}

This study was approved by the Institutional Review Board for Human Subjects Research (IRB-HSR) of the University of Virginia. Clinical enrollment was based on a prospective evaluation of pre-procedure CMR in patients undergoing CRT; the CMR protocol is detailed below. Six patients underwent clinically indicated CT imaging during the CRT implantation follow-up period. For the patients validated with CT imaging, the axial image showing the LV lead tip was compared to the CMR axial images to find the closest match, and then the "true" lead tip location was selected in this CMR axial image. Finally, the lead position error was calculated as the distance between the CT lead tip and projected lead position. For the patient validated by CMR CS venography $(n=1)$, the cardiac veins were manually segmented from MR image slices using custom Matlab software and visually verified to correspond to the implantation vein visible in fluoroscopic venograms.

\section{Fluoroscopy Imaging Protocol}

The implantation procedure for CRT devices was guided by fluoroscopy using a standard system designed for use in the electrophysiology laboratory. The fluoroscopic camera typically was rotated over a range of 80 degrees during the procedure - from 40 degrees in the RAO view to 40 degrees in the LAO view. The SID and table height may also be adjusted independently in order to optimize the fluoroscopic views and minimize scatter. During the procedure, fluoroscopy was used to visualize the leads in the heart, as well as the course of the coronary sinus and its branches. This venography of the coronary sinus was accomplished with an injection of intravenous contrast through a long sheath introduced via the axillary venous system and advanced into the coronary vein. Based on this coronary sinus venography, specific venous branches of the 
coronary sinus were targeted for the coronary sinus pacing lead. After implantation, images of the final lead position were acquired at the two orthogonal views in which the lead position and cardiac anatomy was best visible. SID, table height, and table position were kept constant for all final images, and were recorded for analysis.

\section{CMR Imaging Protocol}

CMR was performed on a 1.5-T clinical scanner (Siemens Avanto) with a phased array receiver coil on the chest. After localization of the heart, 8 to 10 contiguous short-axis slices were prescribed to cover the entire LV from base to apex. Cine images were acquired using a steady-state free precession pulse sequence in each short-axis slice (temporal resolution: 37.9 to $39.5 \mathrm{~ms}$, echo time: $1.3 \mathrm{~ms}$, flip angle: $\alpha=54$ to $73^{\circ}$, field of view: 24 to $28 \mathrm{~cm}$, spatial resolution: 1.27 to $1.48 \mathrm{~mm}$ per pixel, slice thickness: $8 \mathrm{~mm})$. In addition, a stack of axial cine images were acquired with slice thickness of $7 \mathrm{~mm}$. Delayed-enhancement images in locations identical to the cine images were acquired 10 to $15 \mathrm{~min}$ after a bolus injection of $0.2 \mathrm{mmol} / \mathrm{kg}$ gadodiamide (Omniscan, GE Healthcare, Buckinghamshire, United Kingdom) in patients with glomerular filtration rates of at least $60 \mathrm{cc} / \mathrm{min}$, and with gadopentetate dimeglumine (Magnevist, Bayer HealthCare Pharmaceuticals, Tarrytown, New York) in selected patients if the glomerular filtration rate was 45 to $60 \mathrm{cc} / \mathrm{min}$. An inversion recovery fast gradient-echo pulse sequence was used for the acquisition. A whole-heart navigator-guided three dimensional protocol (temporal resolution: $650 \mathrm{~ms}$, echo time: $2.38 \mathrm{~ms}$, flip angle: $\alpha=15^{\circ}$, field of view: 244 $\mathrm{mm}$, spatial resolution: $1.27 \mathrm{~mm}$ per pixel) was used to perform CMR venography of the coronary sinus in selected patients. The gadolinium dose $(0.15 \mathrm{mmol} / \mathrm{kg})$ was infused over 8 minutes during whole heart imaging. 


\section{CT Imaging Protocol}

In the six patients described above with a clinical indication for a post-CRT CT scan, standard cardiac CT imaging was performed using a 128-detector equivalent dual source CT scanner (Siemens Definition, Erlangen Germany) an average of 286 days (range 62-530 days) after CRT implantation. A total of $80 \mathrm{cc}$ of iodinated contrast agent (Visipaque $^{\mathrm{TM}}$ (iodixanol), Amersham Health, Princeton, $\mathrm{NJ}$ ) was administered, followed by a $40 \mathrm{cc}$ saline flush. Typical imaging parameters were: slice thickness $0.625 \mathrm{~mm}$, tube voltage $120 \mathrm{kV}$, and tube current $550 \mathrm{~mA}$,

\subsection{Results}

Results from the eight validation studies are summarized in Table 7.1. Validation was performed first using a canine model with radiopaque beads sewn to the heart muscle to mark the anatomic landmarks and the left ventricular lead sewn to the epicardium. As shown in Figure 7.4A and 7.4B, the artifact seen on CMR SSFP imaging was confirmed to be in the same location as the pacing lead in post-mortem analysis. The radiopaque beads are easily seen on fluoroscopy (panel C) at the RV anterior basal septum (as a substitute for the CSO used in clinical patients), the RV apical septum, and the LMA (180 degrees from the CSO on the mitral annulus). When the lead registration algorithm was applied, the predicted site for the LV lead was within $1.2 \mathrm{~mm}$ (Table 7.1) of the lead artifact visible in CMR SSFP imaging (panel D).

Based on this proof-of-concept study, these methods were then tested in seven patients with pre-CRT CMR imaging. The final lead position and fluoroscopic image angles used for each subject are listed in the Table 7.1. Image angles were selected for optimal view of all landmarks without table adjustment. We measured the difference in 
distances between landmark pairs measured in fluoroscopy and CMR as an estimate of landmark selection error, as shown in Table 7.1. Average difference in landmark distances was $10.0 \pm 7.3 \mathrm{~mm}$.

In the six patients with lead position validated based on postoperative CT scans, we were able to track the course of the lead on the 3D CT images as shown in Figure 7.4A. In all patients, the difference between the predicted location of the LV lead using the algorithm and the actual location of the lead as determined from the CT images was very small, as shown in Table 7.1, with an average distance between the predicted lead position based on our methodology and the actual lead position based on the CT of 14.5 $\pm 10.6 \mathrm{~mm}$. Five of six patients had registration errors less than $15 \mathrm{~mm}$. Figure 7.5 shows an example case, subject CT5, in which the lead placement error was within $6 \mathrm{~mm}$.

In the patient shown in Figure 7.6 with validation based on CMR CS venography, the LV lead was implanted in a branch of the MCV, which is seen on CMR venography (panel A) and fluoroscopically during contrast venography of the coronary sinus at the time of the procedure (panels $B$ and $C$ ). The close proximity between the branch of this vein containing the LV lead as visualized on CMR venography and the predicted LV lead position using the lead localization algorithm is shown in panel $\mathrm{D}$. The distance from the predicted LV position using this method to the MCV branch was $13.3 \mathrm{~mm}$ (Table 7.1).

An example of the future use of this method is shown in Figure 7.7. Scar location, size, and transmurality measured from LGE images is shown on a 2D Hammer projection map. The scar is posterolateral, transmural in the center, and covers $35 \%$ percent of the LV surface. The LV lead is clearly positioned distant from the scar, on the anterior wall. Similar mapping with LV lead position may be completed with maps of electrical activation or mechanical function. 


\subsection{Discussion}

\subsubsection{Advantages of Coregistration Method}

The remarkable features of this method for fluoroscopic and CMR coregistration for LV lead position are: 1) achievement of accurate identification of 3D lead position within 1-2 $\mathrm{cm}$ of the actual lead position based on validation with three different methods (direct pathologic evaluation in a canine, postoperative CT, and CMR venography); 2) wide applicability to any CRT procedure performed in a standard digital fluoroscopy suite as long as fluoroscopic images in just two views are saved; 3) no requirement for additional expensive mapping equipment during the procedure; 4) error minimization algorithm helps alleviate the challenge of fluoroscopic landmark selection; and 5) no need for the radiation exposure, contrast, and expense associated with CT scans.

\subsubsection{Clinical Implications}

CRT can result in major clinical improvements and longer expected survival in appropriately selected patients with heart failure, but there is a persistent nonresponse or suboptimal response rate of approximately $35 \%$ for this procedure. Lead position has been shown to be an important determinant of CRT response. In the CRT nonresponder, it is critical to determine whether suboptimal LV lead position is the cause of the poor response because another lead position can usually be achieved either with a surgical epicardial approach and a repeat endocardial implant procedure.

At the present time, most CRT procedures are performed using standard fluoroscopy rather than navigation using advanced 3D mapping systems. If the patient has had a CMR prior to implant, our methodology enables assessment of lead location 
relative to scar and mechanical activation sequence. This may influence whether a patient who does not respond to CRT is referred for reimplantation of the LV lead in another CS branch, a surgically placed epicardial lead, or evaluation for other therapies such as left ventricular assist device or heart transplant. In addition, at this time, while it seems intuitive that the best LV lead location should be in late-activated ventricular myocardium away from scar, the actual optimal method for determining where the LV lead should be placed may be more complex than this. In this regard, our methodology could serve as an important research tool to validate different rules and algorithms for determination of optimal LV lead placement relative to CMR findings.

\subsubsection{Design of Coregistration Method}

Fluoroscopic imaging during cardiac catheter-based interventions provides excellent real-time visualization of implanted devices and coronary anatomy, but poor visualization of soft tissues. CMR, on the other hand, shows soft tissues with excellent resolution but cannot be performed after device implantation. Registration between these modalities provides complementary information but is especially difficult for cardiac applications because identifying fluoroscopic anatomic landmarks in two views can be challenging, and cardiac and respiratory motion make external markers prone to error. These difficulties have been addressed in previous studies in a number of ways. One strategy describes a dual fluoroscopy and CMR hardware suite, ${ }^{157}$ where the imaging equipment is specially calibrated so that anatomic landmarks are not necessary for coregistration. This method, while time and cost intensive, is meant for live registration during the procedure and can locate a fluoroscopic point in CMR with error

of approximately $5 \mathrm{~mm}$. Another strategy accomplishes coregistration using anatomic 
landmarks derived from fluoroscopic venograms and CMR whole-heart vein imaging. ${ }^{154}$ After manual registration of images, the distance between fluoroscopic and CMR venogram centerlines was less than $2 \mathrm{~mm}$. This method does not require an additional hardware suite, but does require manual registration during the implantation procedure

and specialized registration software installed on the fluoroscope. A similar method implemented with left atrial landmarks measured with a 3D navigator system found coregistration errors around $2 \mathrm{~mm}$ as well. ${ }^{158}$

While these methodologies are very accurate, they are often not available for post-procedure evaluation of CRT nonresponders. Integrated suites and 3D navigator systems are only available at select centers, and even where available they are not routinely used in every patient. Our methodology requires only a pre-procedure CMR study, the acquisition of two fluoroscopic images at the same table position at the end of the implantation procedure, and a record of procedure SID and table height. With this information, our algorithm achieves successful coregistration based on the principle of achieving similar distances between lead position and landmarks on fluoroscopy and CMR. While our average coregistration error of $14.5 \mathrm{~mm}$ is higher than these previouslyreported studies, we believe this method is accurate enough to compare lead position to regional ventricular anatomy and function while requiring much less time, cost, and expertise. As a result, this information can be routinely collected from every CRT patient for post-procedure evaluation or research purposes.

\subsubsection{Limitations}

Our sample size was limited by the number of CRT patients who were also referred for a CT scan. Although there were a limited number of patients studied, we found that this 
was an adequate number to achieve consistent results, which made it difficult to justify performing CT scans on additional patients solely for research purposes. Our validation in a canine study further confirms the accuracy of our algorithm independent of landmark selection.

Coregistration errors were due to both error in landmark selection and errors in validating the LV lead position. Landmark selection from fluoroscopic images was limited by the visibility of soft tissues and the cardiac anatomy. The location of the LMA landmark could be more difficult to determine if the LV lead did not travel all the way to the lateral side, in which case the landmark selection relied solely on venograms acquired before final lead placement. While the landmarks were more visible in CMR images, landmark selection had limited resolution in the through-plane direction, as most images were acquired with slice thickness of $7-8 \mathrm{~mm}$. While the CT scan provides independent validation of the coregistration process, the resolution in matching CT axial images to CMR axial images with axial slice thickness of $7 \mathrm{~mm}$ was limited. In addition, the CT scans were acquired two months to two years post-CRT implant, and in these two years significant cardiac remodeling may occur. CMR venography provided validation without the influence of remodeling, but the final lead placement in the vein can only be estimated in these images.

\subsubsection{Conclusions}

The method presented here permits accurate determination of LV lead position in 3D CMR coordinates based on just two 2D fluoroscopic views from a standard fluoroscopic suite. In this way, it facilitates assessment of myocardial characteristics (scar and mechanical activation) at the LV lead implant site based on the pre-procedure MRI 
without the need for additional equipment during the procedure or the cost, radiation exposure, and contrast associated with a postoperative CT scan. In addition to offering clinicians an early assessment after the CRT procedure of the likelihood that a favorable LV lead position was achieved, the methodology also has potential clinical utility for the CRT nonresponder who might benefit from another procedure to achieve an alternative lead position. Furthermore, this methodology also promises to be useful from a research standpoint to test prediction rules and algorithms for the optimal LV lead position that could eventually be integrated into a navigated CRT procedure based on CMR data. 
Difference in Distance

Between Landmarks ( $\mathrm{mm}$ ) Registration

Os- Os- LMA- Difference

Room Image Angles Location LMA Apex Apex (mm)

\begin{tabular}{|c|c|c|c|c|c|c|c|}
\hline Dog & 1 & RAO $30^{\circ}$, LAO $30^{\circ}$ & L & 0.1 & 15.9 & -10.9 & 1.2 \\
\hline CT1 & 2 & RAO $25^{\circ}$, LAO $25^{\circ}$ & $A L$ & 17.4 & 15.4 & 0.7 & 9.2 \\
\hline CT2 & 2 & AP $0^{\circ}$, LAO $30^{\circ}$ & $\mathrm{AL}$ & -0.7 & 16.2 & 4.5 & 35.3 \\
\hline CT3 & 2 & AP $0^{\circ}$, LAO $30^{\circ}$ & AS & -8.1 & -6.9 & 2.0 & 10.6 \\
\hline CT4 & 2 & RAO $25^{\circ}$, LAO $25^{\circ}$ & $A$ & 12.9 & -8.8 & 18.2 & 11.3 \\
\hline CT5 & 3 & RAO $30^{\circ}$, LAO $30^{\circ}$ & $A L$ & 9.3 & 17.7 & 2.7 & 5.8 \\
\hline CT6 & 1 & $\mathrm{RAO} 25^{\circ}, \mathrm{AP} 0^{\circ}$ & A & -3.6 & -5.4 & -8.4 & 14.6 \\
\hline V1 & 4 & RAO 28 $8^{\circ}$, LAO $25^{\circ}$ & $P$ & 12.0 & 9.8 & 30.0 & 13.3 \\
\hline
\end{tabular}

$\mathrm{L}=$ lateral wall $\mathrm{AL}=$ anterolateral wall; $\mathrm{AS}=$ anteroseptal wall; $\mathrm{A}=$ anterior wall; $\mathrm{P}=$ posterior wall; CT1-CT6 = patients with CT imaging, V1 = patient with CMR venogram. Differences between landmark distances were computed as fluoroscopic distance minus CMR distance. 
Pre-Study

Calibration

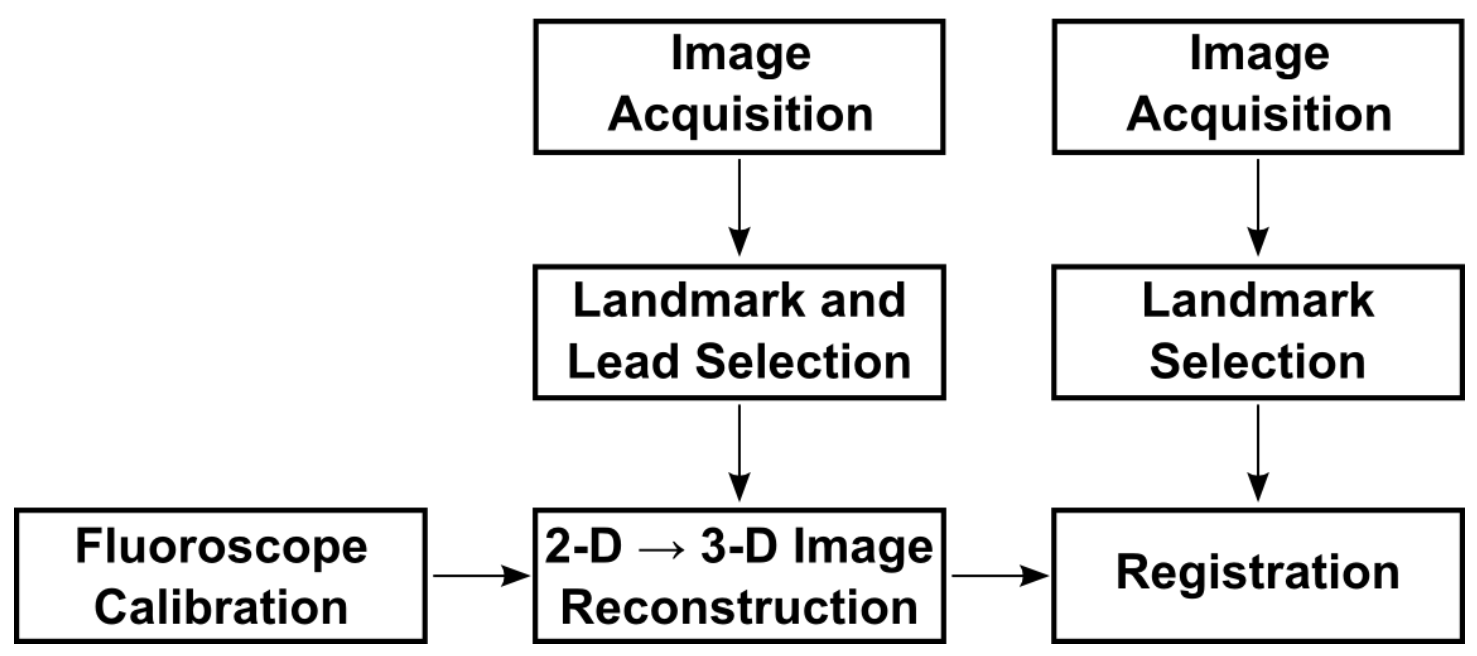

Figure 7.1 : Process flow diagram for registering fluoroscopic images to CMR images.

Image registration requires pre-study calibration (column 1), 2D fluoroscopic imaging (column 2), and 3D CMR imaging (column 3). 2D fluoroscopic images are acquired at minimum two angles, and landmarks and the LV lead tip are selected from each image. Pre-study image calibration is applied to reconstruct these 2D fluoroscopic landmarks into 3D coordinates. Meanwhile, landmarks are selected from pre-procedure 3D CMR images. Finally, the registration process merges the fluoroscopic and CMR coordinate systems using the common landmarks. 


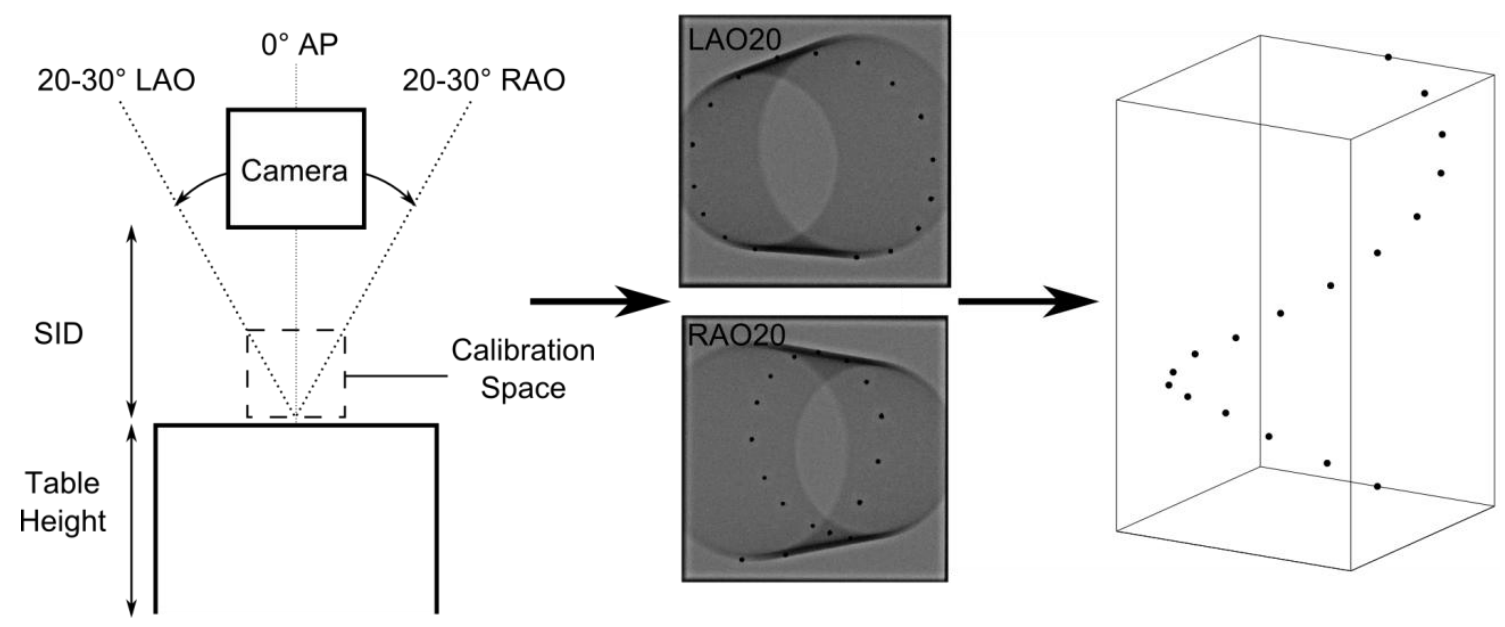

Figure 7.2 Calibration to transform 2D fluoroscopic data to 3D coordinates

The fluoroscopy equipment was calibrated for standard $L A O, R A O$, and AP view angles at a standard SID and table height (A). Two 2D fluoroscopic images of a cylindrical phantom with radiopaque beads along its length $(\mathbf{B})$ were used to calculate the relationship between the bead coordinates in the image and the real 3D coordinates with respect to the origin bead. This calibration was used to reconstruct three $2 \mathrm{D}$ fluoroscopic views into the bead coordinates in 3D (C). 

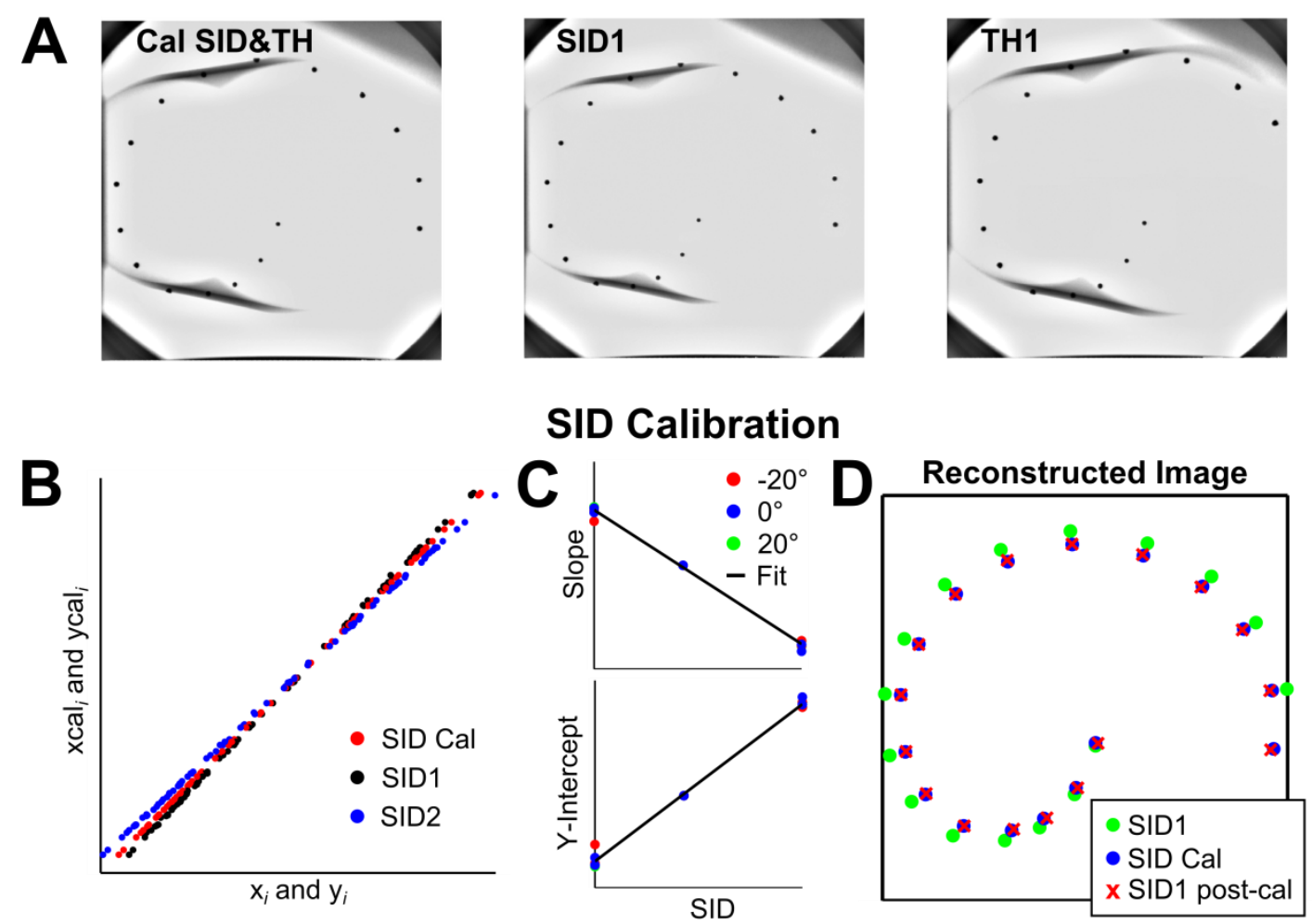

\section{Table Height Calibration}
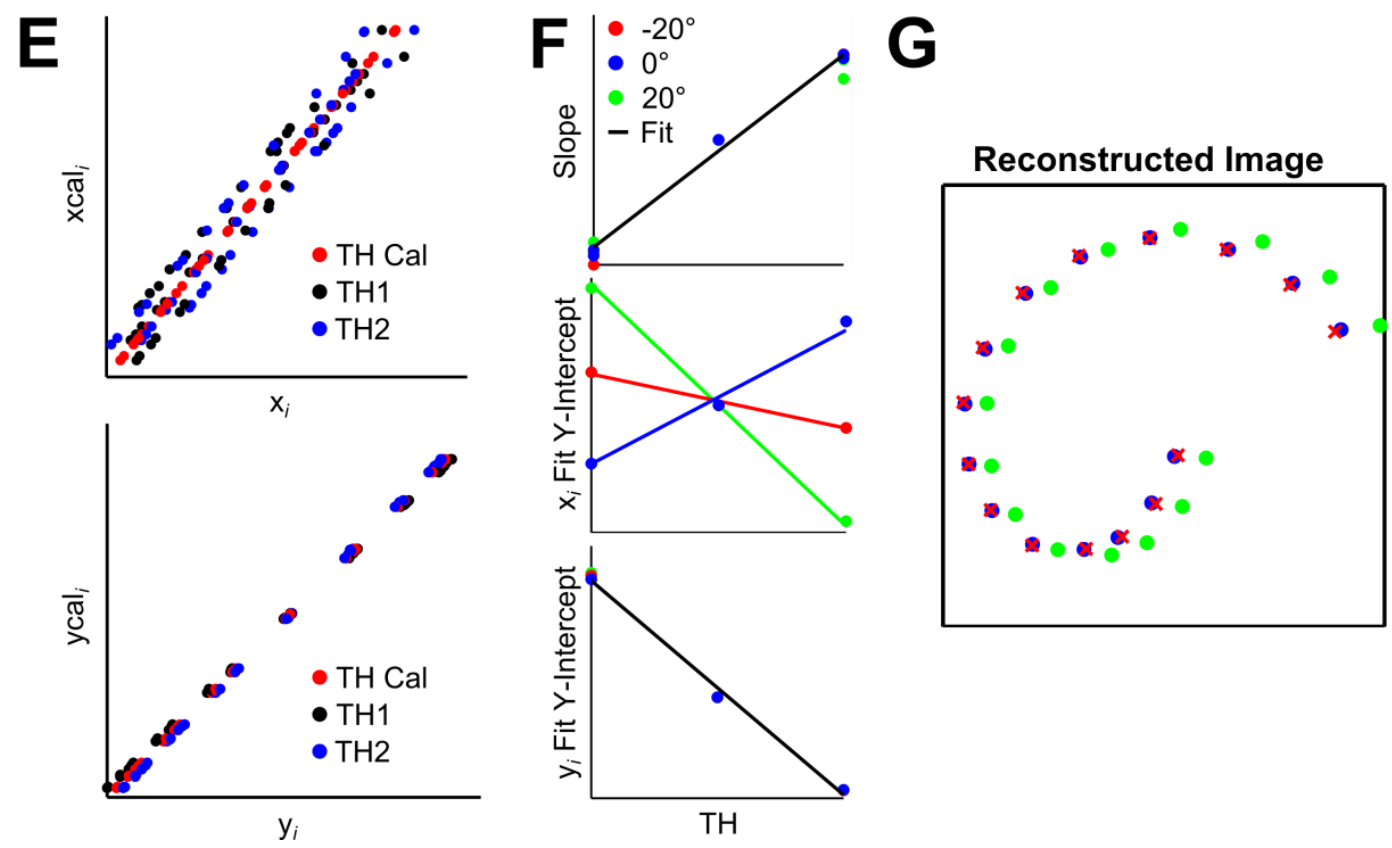

Figure 7.3 Fluoroscopic magnification adjustment

See caption on next page. 
Figure 7.3: Images were acquired at multiple SID and table height (TH) values to determine the relationship between object coordinates in a magnified image $\left(x_{i}\right.$ and $y_{i}$, acquired at SID1 or TH1) and object coordinates in an image acquired at the calibration SID or TH $\left(\right.$ xcal $_{i}$ and $\left.y c a l_{i}\right)$. Images at multiple SID and TH values were acquired without moving the table (A). The relationship between $x_{i}$ and $y_{i}$ at SID1 and $x_{c a l}$ and $y c a l_{i}$ at the calibration SID is linear (B). The slope and Y-intercept of this relationship varied with SID but was the same for every acquisition angle and for both $\mathrm{x}$ and $\mathrm{y}$ coordinates $(\mathbf{C})$. This linear fit can be used to correctly reconstruct the image acquired at SID1 had it been acquired at the calibration SID (D). A similar process was used to reconstruct images acquired at different table heights. However, while $y_{i}$ is clearly linearly related to $y_{c a l} i_{i}$ $\mathrm{xcal}_{i}$ is not just a function of table height and $\mathrm{x}_{i}(\mathbf{E})$. The Y-intercept of the $\mathrm{x}_{i} \mathrm{xcal}_{i}$ relationship is a function of both $\mathrm{TH}$ and acquisition angle, which was accounted for with a multiple linear regression fit $(\mathbf{F})$. This process allowed accurate reconstruction of the bead position at the calibration $\mathrm{TH}(\mathbf{G})$. 


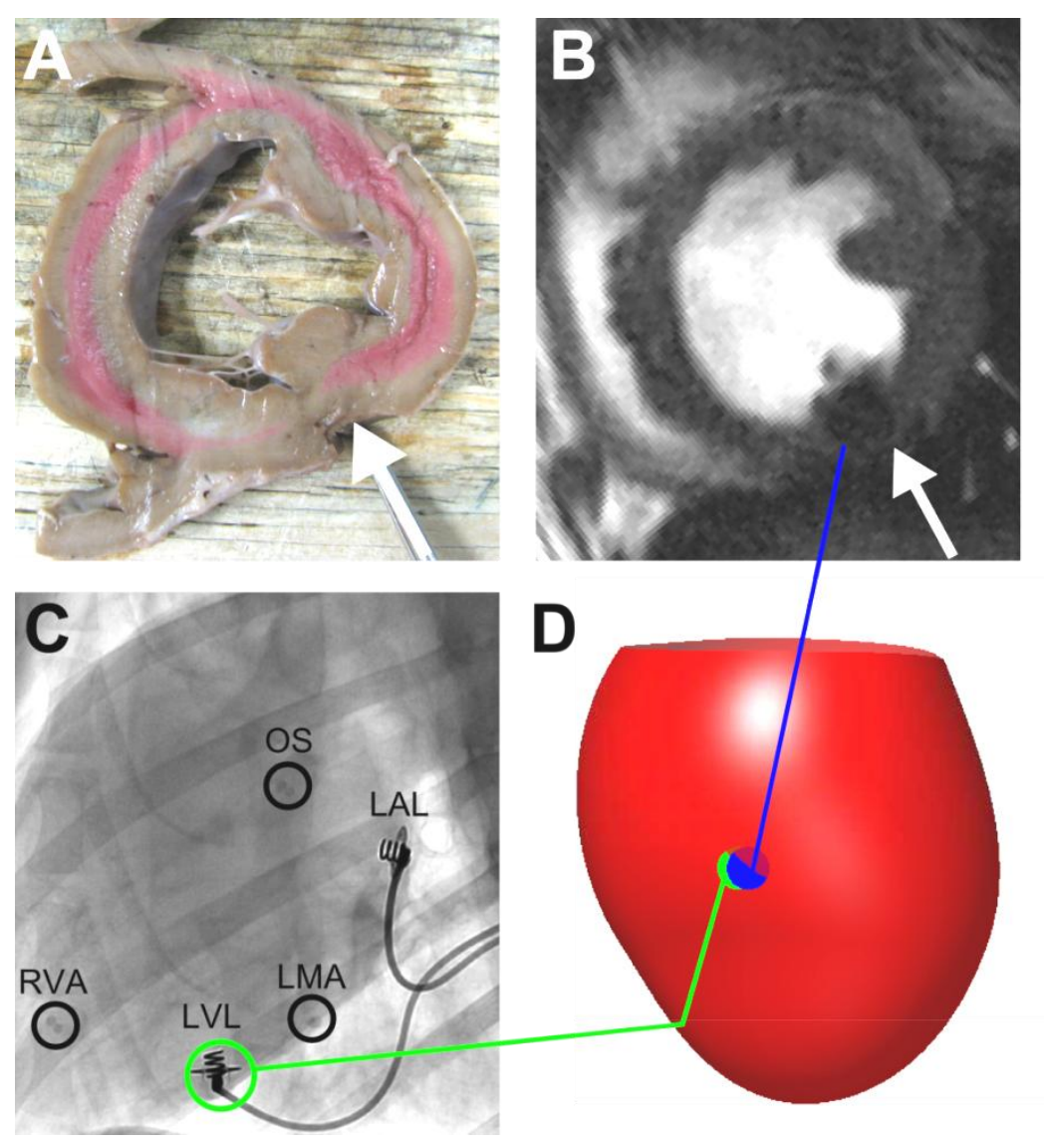

Figure 7.4 Canine validation of lead localization algorithm

The short-axis cross-section of the left ventricle containing the epicardial LV lead for this canine was dissected during post-mortem analysis $(\mathbf{A})$. The artifact produced by the lead tip can be seen in the corresponding short-axis slice from the CMR study (B). The mild difference in the endocardial contours is due to the way the explanted left ventricle was sliced. In an example fluoroscopic image from this canine, beads are used to mark the coronary sinus os (OS), right ventricular apex (RVA), and lateral mitral annulus (LMA) (C). The epicardial left ventricular lead (LVL) and an epicardial left atrial lead (LAL) are also marked. An anterolateral view of an integrated 3D map showing the epicardium (red surface) demonstrates the proximity of the predicted LV lead position from fluoroscopy (green) to the LV lead position measured from CMR (blue) (D). 

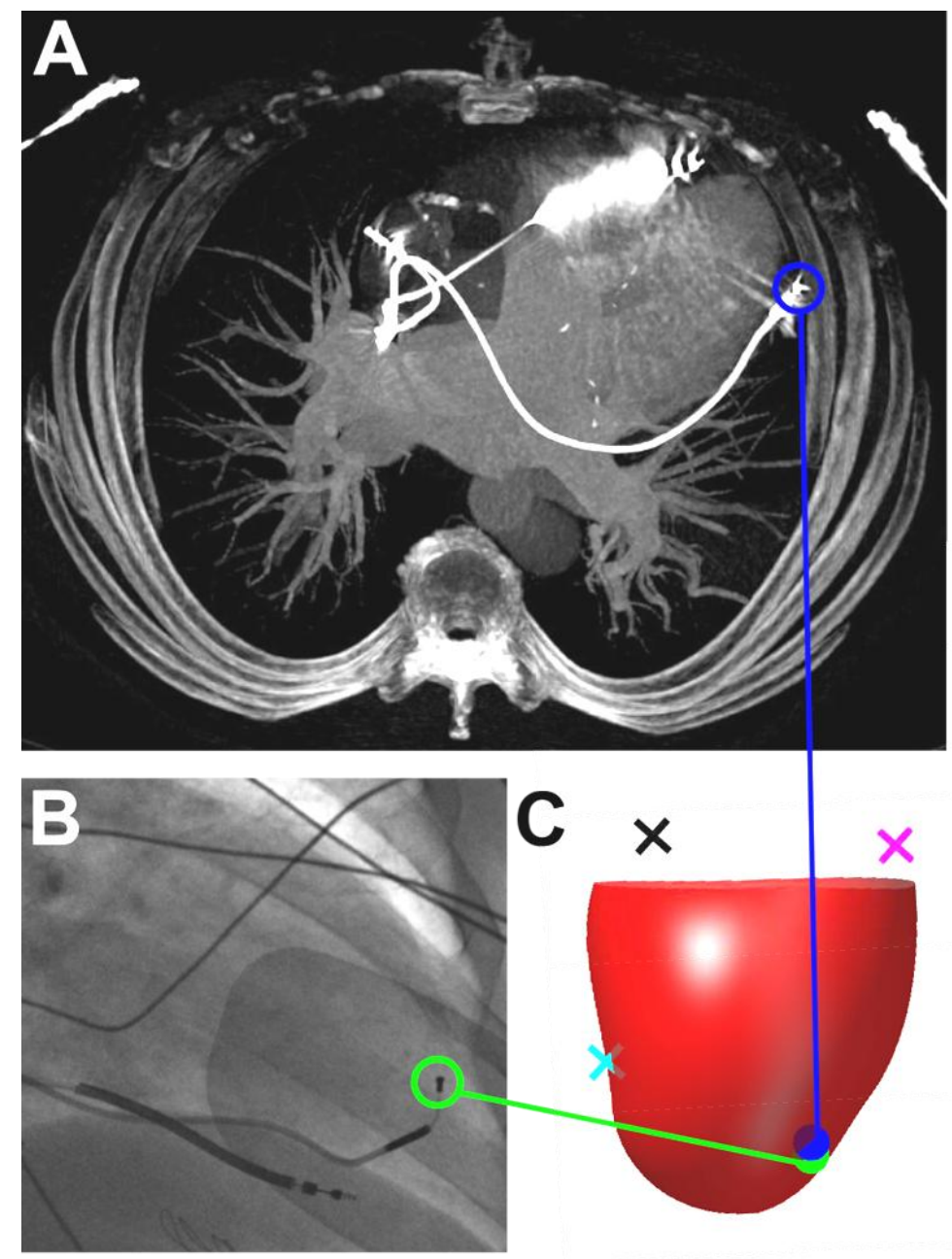

Figure 7.5 Validation of lead localization algorithm based on Cardiac Computed Tomography

The actual location based on CT imaging of the final LV lead position is shown (A) and compared with the fluoroscopic image of the final LV lead position (B) for the subject CT5. An anterior view of a 3D integrated map shows the epicardium (red surface) with the RVA (cyan), LMA (magenta), and CSO (black) landmarks (C). The predicted location of the LV lead (green) using the algorithm is very close compared with the lead position in CT (blue). 


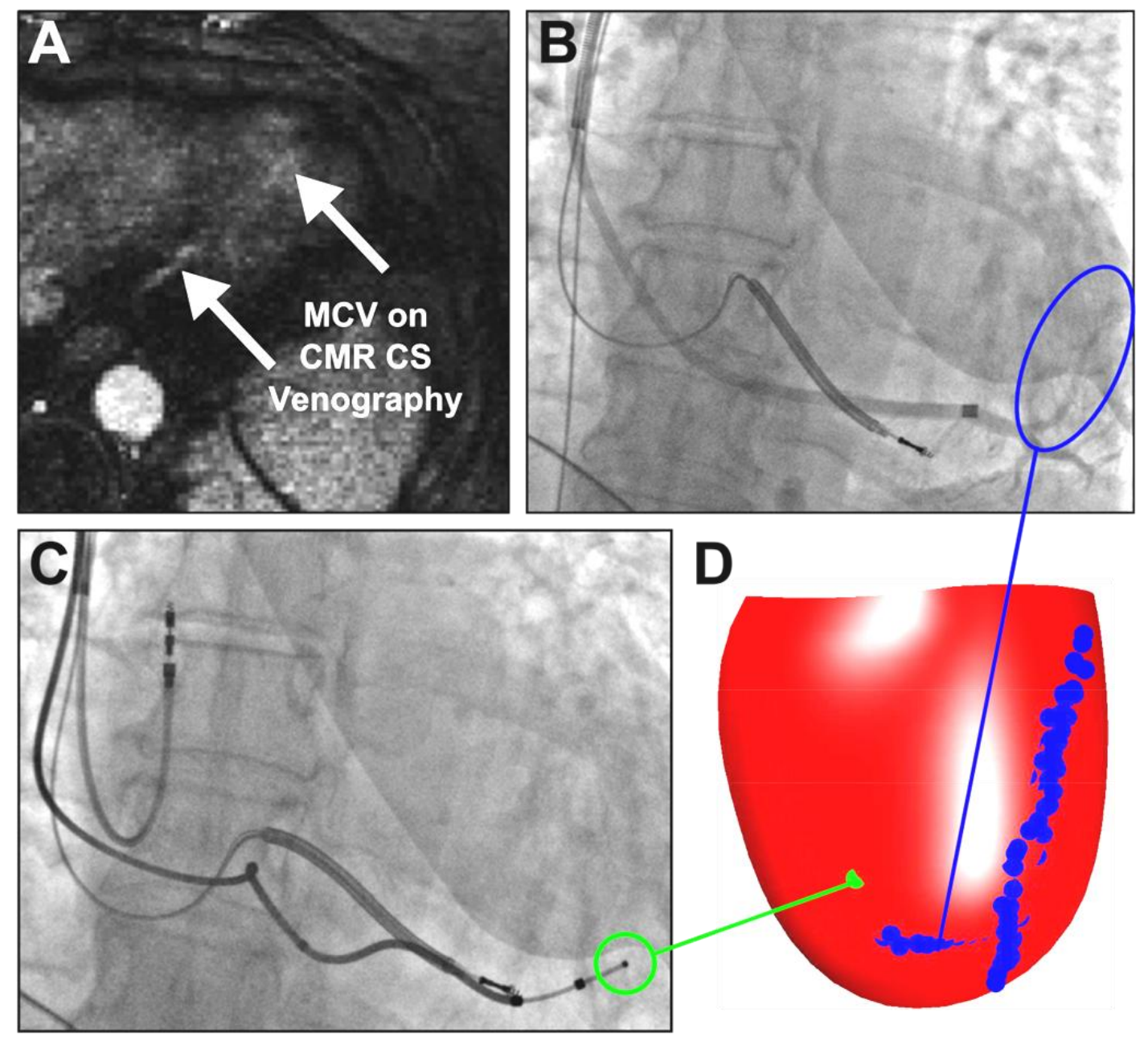

Figure 7.6 Validation of lead localization algorithm based on CMR venography of the coronary sinus

The middle cardiac vein (MCV) is seen in gadolinium-enhanced CMR venography using a whole-heart navigator-guided 3D protocol $(\mathbf{A})$. The branch of the MCV in which the LV lead was eventually implanted is shown fluoroscopically with contrast enhanced venography $(\mathbf{B})$, and the actual final LV lead position is shown in the same view $(\mathbf{C})$. After registration, the LV lead position (green) and the MCV segmented manually from the CMR (blue) are shown on a lateral view of a 3D mesh representing the CMR epicardial contours (D). 


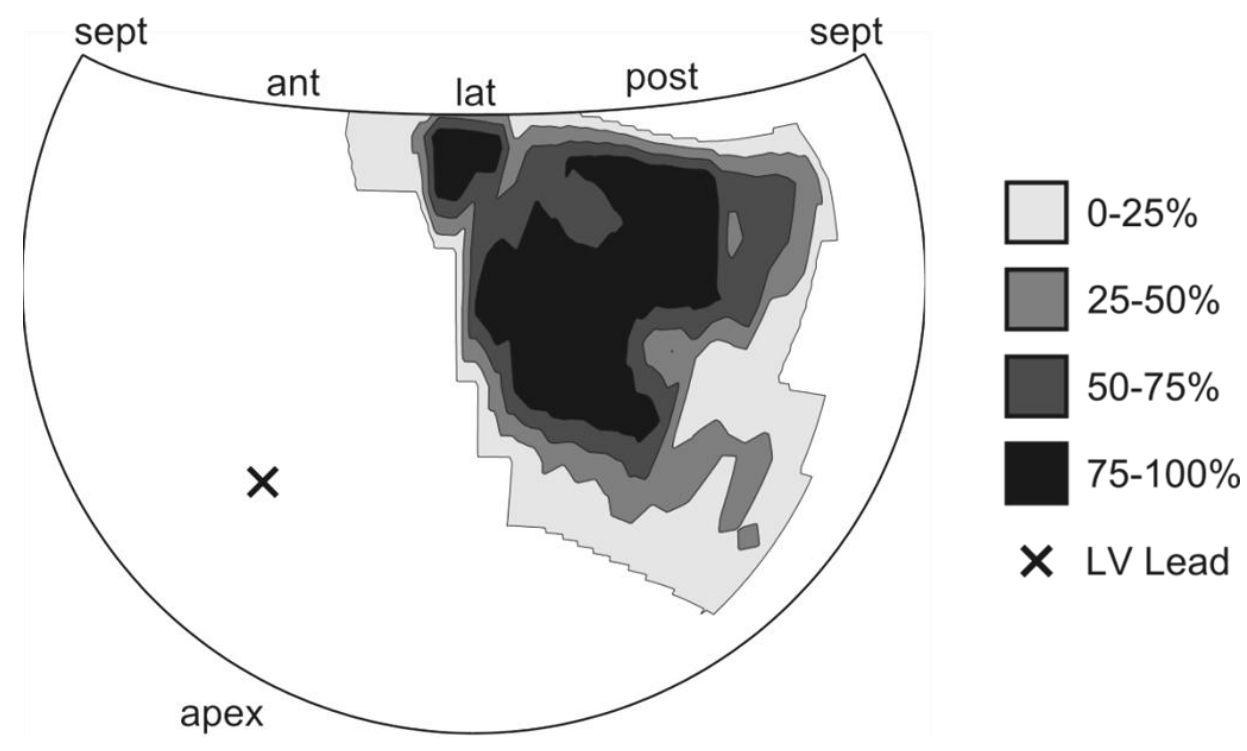

Figure 7.7 Integrated map of LV lead position and myocardial scar

Scar transmurality, as a percentage of short axis wall thickness, was measured from LGE CMR short axis slices and mapped on a 2D Hammer projection map of the LV surface. LV lead location, measured from the fluoroscopy registration method, is shown to be remote from the scar. 


\section{Future Directions}

In this dissertation, quantitative measures of regional myocardial mechanics were evaluated for their ability to detect and measure the size and location of a region of demand ischemia during dobutamine stress testing. We found that both area strain and three-dimensional fractional shortening (3DFS) were sensitive to ischemic region force generation in finite element (FE) modeling and detected critical coronary stenosis in experimental studies with high sensitivity and specificity. In initial clinical studies, 3DFS showed low variability in patients without coronary stenosis and thus appears promising for measuring wall motion abnormalities caused by coronary stenosis. Finally, these methods for mapping cardiac geometry were used to develop new methods for measuring the location of a cardiac resynchronization therapy (CRT) lead with respect to left ventricular anatomy and scar. The methods developed here provide new and more quantitative information to enhance both echocardiography stress testing and CRT implantation, and may be applied in a number of future directions.

\subsection{Demand Ischemia Border Zone Mechanics}

The FE modeling study detailed in Chapter 3 concluded that the size of the region with abnormal radial strain during demand ischemia is smaller than the region with abnormal circumferential or longitudinal strain. The FE model can be used in the future to determine the mechanism that causes this effect. Wall thickness was slightly decreased in the border zone outside of the ischemic region during baseline, while wall thickness did not decrease until inside of the ischemic region with increased global contractility in 
the presence of dobutamine. We hypothesize that the circumferential-radial and longitudinal-radial shear stiffness of the activated myocardium increases with increased contractility, and this increased stiffness opposes radial systolic thinning in the border zone. This shear stiffness is not explicitly included in the material property models, but we hypothesize that stiffening fibers in the circumferential-longitudinal plane increases the "structural stiffness" in the radial direction. This hypothesis can be tested by implementing fully activated myocardial material properties and fiber angles into a simple cube FE model, and then testing the shear stiffness of this model. If the shear stiffness increases with peak active force generation, this would provide more evidence that this stiffness is the underlying mechanism for the different abnormality sizes observed for radial strain during demand ischemia.

Finally, while border zone behavior has been extensively studied in experimental models of supply ischemia and infarcts, there are very few experimental studies that measure border zone mechanics during demand ischemia. Both the modeling and experimental results of this dissertation suggest that border zone mechanics during demand ischemia are much different than supply ischemia, and that these differences significantly change the relationship between the size of mechanical abnormality and the size of a perfusion abnormality. Our results in Chapter 3 suggest that radial strain in the border zone may display different patterns between demand and supply ischemia. It would be interesting to test these results in vivo to evaluate the effect of fiber sheet geometry, which was not included in the model, on radial strain and wall thickening during dobutamine stress. A detailed experimental analysis of border zone mechanical behavior during demand ischemia would help increase understanding of the relationship between mechanical and perfusion abnormality sizes, which is essential if diagnostic 
information beyond the presence or absence of ischemia is to be measured from dobutamine stress testing..

\subsection{Clinical Quantitative Wall Motion Analysis}

In Chapter 6, $\triangle 3$ DFS was measured in patients who were referred for dobutamine stress echocardiography but were at low risk for coronary artery disease. The low variability in these patients suggests that $\triangle$ BDFS may be effective for detecting coronary stenosis. However, $\triangle 3$ DFS must be measured in patients with coronary stenosis who later undergo angiography to determine its sensitivity and the optimum threshold for detecting a wall motion abnormality. Therefore, the next direction in this work is enrolling patients with coronary stenosis in a clinical study of $\triangle 3$ DFS. In light of our results from Chapters 3 and 4 indicating the utility of area strain for detecting coronary stenosis, we also propose performing speckle tracking analysis on the 3D images acquired for this study. By acquiring strain over the entire LV surface, strain could be compared to $\triangle$ BDFS for both detecting coronary stenosis and for measuring the size of the wall motion abnormality. Further, radial strain could be compared to circumferential and longitudinal strain to compare the size of strain abnormalities in the three directions in patients. The quality of this comprehensive $3 D$ strain analysis would depend on the quality of $3 D$ echocardiography system and strain analysis software available, as measuring radial strain from 3D speckle tracking has significant variability which depends on image quality. ${ }^{159}$ This data set would provide a comprehensive comparison of 3D strain and 3D wall motion analysis in clinical cases, with inherent population and measurement variability. This data would provide definitive evidence and thresholds for implementing 
quantitative assessment of wall motion abnormalities during dobutamine stress in a clinical setting.

\subsection{Quantitative Wall Motion Analysis of Infarction}

The results detailed in Chapter 5 showed that dyskinesia severity index (DSI) was too variable to separate images of healed myocardial infarcts from images acquired 48 hour post-occlusion. However, modeling results indicated that DSI may be effective for detecting infarcts during stress testing. These results must be tested in vivo, as the amount of variability will determine the utility of DSI during stress testing just as the variability limited DSI in images acquired at rest. In addition, while infarcts appeared to be less stiff than partially-activated regions of demand ischemia in the model, this effect could be a result of our methods for modeling demand ischemia and infarcts. If a region of demand ischemia or infarct is non-transmural, for example, the relative stiffness of these two regions may be different. A significant number of patients who undergo clinical stress testing have prior myocardial infarction, and so images of patients with prior infarcts during dobutamine stress testing may be acquired as part of the clinical study suggested above to test the ability of DSI to separate wall motion abnormalities at peak stress caused by prior infarcts from those caused by demand ischemia. 


\subsection{Application of Lead Mapping to Evaluation of Cardiac Resynchronization Therapy Nonresponders}

The method developed in Chapter 7 was shown to determine CRT lead location accurately with respect to geometry measured from pre-procedure CMR images. The next step of this work is to apply this method to evaluate CRT nonresponders. Lead placement close to infarcted myocardium is thought to reduce CRT efficacy. ${ }^{151,152,160}$ Using this new method for measuring lead placement, this hypothesis can be tested directly by measuring lead location in patients who have received late gadoliniumenhanced imaging before CRT implantation. These results will enable better evaluation of the reasons for CRT nonresponse in both research studies and in planning treatment options for individual patients. 


\section{References}

1. Roger VL, Go AS, Lloyd-Jones DM, Benjamin EJ, Berry JD, Borden WB, Bravata DM, Dai S, Ford ES, Fox CS, Fullerton HJ, Gillespie C, Hailpern SM, Heit J a, Howard VJ, Kissela BM, Kittner SJ, Lackland DT, Lichtman JH, Lisabeth LD, Makuc DM, Marcus GM, Marelli A, Matchar DB, Moy CS, Mozaffarian D, Mussolino ME, Nichol G, Paynter NP, Soliman EZ, Sorlie PD, Sotoodehnia N, Turan TN, Virani SS, Wong ND, Woo D, Turner MB. Heart disease and stroke statistics--2012 update: a report from the American Heart Association. Circulation. 2012; 125:e2-e220.

2. Ford ES, Ajani U a, Croft JB, Critchley J a, Labarthe DR, Kottke TE, Giles WH, Capewell S. Explaining the decrease in U.S. deaths from coronary disease, 1980-2000. N Engl J Med. 2007; 356:2388-98.

3. O'Connor RE, Brady W, Brooks SC, Diercks D, Egan J, Ghaemmaghami C, Menon V, O’Neil BJ, Travers AH, Yannopoulos D. Part 10: acute coronary syndromes: 2010 American Heart Association Guidelines for Cardiopulmonary Resuscitation and Emergency Cardiovascular Care. Circulation. 2010; 122:S787-817.

4. Feldman DN, Gade CL, Slotwiner AJ, Parikh M, Bergman G, Wong SC, Minutello RM. Comparison of outcomes of percutaneous coronary interventions in patients of three age groups (<60, 60 to 80, and $>80$ years) (from the New York State Angioplasty Registry). Am J Cardiol. 2006; 98:1334-9.

5. Boden W, O'Rourke R, Teo K, Hartigan P, Maron D, Kostuk W, Knudtson M, Dada M, Casperson P, Harris C, Chaitman B, Shaw L, Gosselin G, Nawaz S, Title L, Gau G, Blaustein A, Booth D, Bates E, Spertus J, Berman D, Mancini G, Weintraub W. Optimal 
medical therapy with or without PCI for stable coronary disease. N Engl J Med. 2007; $356: 1503-1516$.

6. Shaw LJ, Berman DS, Maron DJ, Mancini GBJ, Hayes SW, Hartigan PM, Weintraub WS, O'Rourke R a, Dada M, Spertus J a, Chaitman BR, Friedman J, Slomka P, Heller G V, Germano G, Gosselin G, Berger P, Kostuk WJ, Schwartz RG, Knudtson M, Veledar E, Bates ER, McCallister B, Teo KK, Boden WE. Optimal medical therapy with or without percutaneous coronary intervention to reduce ischemic burden: results from the Clinical Outcomes Utilizing Revascularization and Aggressive Drug Evaluation (COURAGE) trial nuclear substudy. Circulation. 2008; 117:1283-91.

7. Tonino PAL, De Bruyne B, Pijls NHJ, Siebert U, Ikeno F, van't Veer M, Klauss V, Manoharan G, Engstrøm T, Oldroyd KG, Ver Lee PN, MacCarthy PA, Fearon WF. Fractional flow reserve versus angiography for guiding percutaneous coronary intervention. N Engl J Med. 2009; 360:213-24.

8. Fihn SD, Gardin JM, Abrams J, Berra K, Blankenship JC, Dallas AP, Douglas PS, Foody JM, Gerber TC, Hinderliter AL, King SB, Kligfield PD, Krumholz HM, Kwong RYK, Lim MJ, Linderbaum JA, Mack MJ, Munger MA, Prager RL, Sabik JF, Shaw LJ, Sikkema JD, Smith CR, Smith SC, Spertus JA, Williams S V. 2012 ACCF/AHA/ACP/AATS/PCNA/SCAI/STS Guideline for the Diagnosis and Management of Patients With Stable Ischemic Heart Disease: A Report of the American College of Cardiology Foundation/American Heart Association Task Force on Practice Guidelines, and the. Circulation. 2012; 60:e1-e164.

9. Gould K, Lipscomb K, Hamilton G. Physiologic Basis for Assessing Critical Coronary Stenosis. Instantaneous Flow Response and Regional Distribution During Coronary 
Hyperemia as Measures of Coronary Flow Reserve. Journal of Cardiology. 1974; 33:8794.

10. Pijls NH, De Bruyne B, Peels K, Van Der Voort PH, Bonnier HJ, Bartunek J Koolen $\mathrm{JJ}$, Koolen JJ. Measurement of fractional flow reserve to assess the functional severity of coronary-artery stenoses. N Engl J Med. 1996; 334:1703-8.

11. Tonino P a L, Fearon WF, De Bruyne B, Oldroyd KG, Leesar M a, Ver Lee PN, Maccarthy P a, Van't Veer M, Pijls NHJ. Angiographic versus functional severity of coronary artery stenoses in the FAME study fractional flow reserve versus angiography in multivessel evaluation. J Am Coll Cardiol. 2010; 55:2816-21.

12. Marzilli M, Merz CNB, Boden WE, Bonow RO, Capozza PG, Chilian WM, DeMaria AN, Guarini G, Huqi A, Morrone D, Patel MR, Weintraub WS. Obstructive coronary atherosclerosis and ischemic heart disease: an elusive link! J Am Coll Cardiol. 2012; $60: 951-6$.

13. Heijenbrok-Kal MH, Fleischmann KE, Hunink MGM. Stress echocardiography, stress single-photon-emission computed tomography and electron beam computed tomography for the assessment of coronary artery disease: a meta-analysis of diagnostic performance. Am Heart J. 2007; 154:415-23.

14. Geleijnse ML, Fioretti PM, Roelandt JR. Methodology, feasibility, safety and diagnostic accuracy of dobutamine stress echocardiography. J Am Coll Cardiol. 1997; 30:595-606.

15. Hoffmann R, Lethen H, Marwick T, Arnese M, Fioretti P, Pingitore A, Picano E, Buck T, Erbel R, Flachskampf FA, Hanrath P. Analysis of interinstitutional observer agreement 
in interpretation of dobutamine stress echocardiograms. J Am Coll Cardiol. 1996; 27:330-6.

16. Picano E, Lattanzi F, Orlandini A, Marini C, L'Abbate A. Stress echocardiography and the human factor: the importance of being expert. J Am Coll Cardiol. 1991; 17:6669.

17. Smith S, Jr HP. High-speed ultrasound volumetric imaging system. I. Transducer design and beam steering. IEEE Trans Ultrason Ferroelectr Freq Control. 2002; 38:100108.

18. Aggeli C, Giannopoulos G, Misovoulos P, Roussakis G, Christoforatou E, Kokkinakis C, Brili S, Stefanadis C. Real-time three-dimensional dobutamine stress echocardiography for coronary artery disease diagnosis: validation with coronary angiography. Heart. 2007; 93:672-5.

19. Sawada SG, Thomaides A. Three-dimensional stress echocardiography: the promise and limitations of volumetric imaging. Curr Opin Cardiol. 2009; 24:426-32.

20. Boland LL, Folsom AR, Sorlie PD, Taylor H a, Rosamond WD, Chambless LE, Cooper LS. Occurrence of unrecognized myocardial infarction in subjects aged 45 to 65 years (the ARIC study). Am J Cardiol. 2002; 90:927-31.

21. Sheifer SE, Gersh BJ, Yanez ND, Ades P a, Burke GL, Manolio T a. Prevalence, predisposing factors, and prognosis of clinically unrecognized myocardial infarction in the elderly. J Am Coll Cardiol. 2000; 35:119-26. 
22. Kwong RY, Chan AK, Brown K a, Chan CW, Reynolds HG, Tsang S, Davis RB. Impact of unrecognized myocardial scar detected by cardiac magnetic resonance imaging on event-free survival in patients presenting with signs or symptoms of coronary artery disease. Circulation. 2006; 113:2733-43.

23. Pellikka PA, Nagueh SF, Elhendy AA, Kuehl CA, Sawada SG. American Society of Echocardiography recommendations for performance, interpretation, and application of stress echocardiography. J Am Soc Echocardiogr. 2007; 20:1021-41.

24. Quinones M a., Verani MS, Haichin RM, Mahmarian JJ, Suarez J, Zoghbi W a. Exercise echocardiography versus $201 \mathrm{TI}$ single-photon emission computed tomography in evaluation of coronary artery disease. Analysis of 292 patients. Circulation. 1992; 85:1026-1031.

25. Tennant R, Wiggers $C$. The effect of coronary occlusion on myocardial contraction. Am J Physiol. 1935; 112:351-361.

26. Gebert G, Benzing H, Strohm M. Changes in the interstitial pH of dog myocardium in response to local ischemia, hypoxia, hyper- and hypocapnia, measured continuously by means of glass microelectrodes. Pflug Arch Eur J Phy. 1971; 329:72-81.

27. Effros RM, Haider B, Ettinger PO, Ahmed Sultan S, Oldewurtel HA, Marold K, Regan TJ. In vivo myocardial cell $\mathrm{pH}$ in the dog. Response to ischemia and infusion of alkali. $\mathrm{J}$ Clin Invest. 1975; 55:1100-10.

28. Stanley WC. Myocardial Energy Metabolism During Ischemia and the Mechanisms of Metabolic Therapies. J Cardiovasc Pharmacol Ther. 2004; 9:S31-S45. 
29. Steenbergen C, Deleeuw G, Rich T, Williamson JR. Effects of acidosis and ischemia on contractility and intracellular pH of rat heart. Circ Res. 1977; 41:849-58.

30. Allen D, Orchard C. Myocardial contractile function during ischemia and hypoxia. Circ Res. 1987; 60:153-168.

31. Orchard C, Kentish J. Effects of changes of $\mathrm{pH}$ on the contractile function of cardiac muscle. Am J Physiol Cell Physiol. 1990; 258:C967.

32. Clarke K, Willis R. Energy metabolism and contractile function in rat heart during graded, isovolumic perfusion using 31P nuclear magnetic resonance spectroscopy. $\mathrm{J}$ Mol Cell Cardiol. 1987; 1160.

33. Vatner SF. Correlation between acute reductions in myocardial blood flow and function in conscious dogs. Circ Res. 1980; 47:201-7.

34. Villarreal FJ, Lew W, Waldman LK, Covell JW. Transmural myocardial deformation in the ischemic canine left ventricle. Circ Res. 1991; 68:368-81.

35. Bogen D, Needleman A, TA M. An analysis of myocardial infarction. The effect of regional changes in contractility. Circ Res. 1984; 55:805-815.

36. Nielsen PM, Le Grice IJ, Smaill BH, Hunter PJ. Mathematical model of geometry and fibrous structure of the heart. Am J Physiol. 1991; 260:H1365-78.

37. Kerckhoffs R, Neal M, Gu Q, Bassingthwaighte J, Omens J, McCulloch A. Coupling of a 3D finite element model of cardiac ventricular mechanics to lumped systems models of the systemic and pulmonic circulation. Ann Biomed Eng. 2007; 35:1-18. 
38. Mazhari R, McCulloch A. Integrative models for understanding the structural basis of regional mechanical dysfunction in ischemic myocardium. Ann Biomed Eng. 2000; 28:979-990.

39. Walker JC, Ratcliffe MB, Zhang P, Wallace AW, Fata B, Hsu EW, Saloner D, Guccione JM. MRI-based finite-element analysis of left ventricular aneurysm. Am J Physiol Heart Circ Physiol. 2005; 289:H692.

40. Mazhari R, Omens J, Waldman L, McCulloch A. Regional myocardial perfusion and mechanics: a model-based method of analysis. Ann Biomed Eng. 1998; 26:743-755.

41. Mazhari R, Omens JH, Covell JW, McCulloch AD. Structural basis of regional dysfunction in acutely ischemic myocardium. Cardiovasc Res. 2000; 47:284.

42. Lee LC, Wenk JF, Klepach D, Zhang Z, Saloner D, Wallace AW, Ge L, Ratcliffe MB, Guccione JM. A novel method for quantifying in-vivo regional left ventricular myocardial contractility in the border zone of a myocardial infarction. J Biomech Eng. 2011; 133:094506.

43. Tuttle R, Mills J. Dobutamine: development of a new catecholamine to selectively increase cardiac contractility. Circ Res. 1975; 36:185-196.

44. Bers DM. Cardiac excitation-contraction coupling. Nature. 2002; 415:198-205.

45. Gambassi G, Spurgeon H a, Lakatta EG, Blank PS, Capogrossi MC. Different effects of alpha- and beta-adrenergic stimulation on cytosolic $\mathrm{pH}$ and myofilament responsiveness to Ca2+ in cardiac myocytes. Circ Res. 1992; 71:870-82. 
46. Schulz R, Miyazaki S, Miller M, Thaulow E, G. Consequences of regional inotropic stimulation of ischemic myocardium on regional myocardial blood flow and function in anesthetized swine. Circulation. 1989; 64:1116-1126.

47. Kroll K, Martin G. Comparison of myocardial ATP, blood flow, and cytosolic adenosine in demand ischemia and coronary occlusion. Am J Physiol Heart Circ Physiol. 1995; 3:H819-28.

48. Van Rossum AC, Visser FC, Sprenger M, Van Eenige MJ, Valk J, Roos JP. Evaluation of magnetic resonance imaging for determination of left ventricular ejection fraction and comparison with angiography. Am J Cardiol. 1988; 62:628-33.

49. Zerhouni EA, Parish DM, Rogers WJ, Yang A, Shapiro EP. Human heart: tagging with MR imaging-a method for noninvasive assessment of myocardial motion. Radiology. 1988; 169:59.

50. O'Dell WG, Moore CC, Hunter WC, Zerhouni EA, McVeigh ER. Three-dimensional myocardial deformations: calculation with displacement field fitting to tagged MR images. Radiology. 1995; 195:829.

51. Aletras a H, Ding S, Balaban RS, Wen H. DENSE: displacement encoding with stimulated echoes in cardiac functional MRI. J Magn Reson. 1999; 137:247-52.

52. Kim D, Gilson WD, Kramer CM, Epstein FH. Myocardial Tissue Tracking with Twodimensional Cine Imaging : Development and Initial Evaluation. Radiology. 2004; 230:862-871. 
53. Zhong X, Helm P a, Epstein FH. Balanced multipoint displacement encoding for DENSE MRI. Magnet Reson Med. 2009; 61:981-8.

54. Van Rugge FP, van Der Wall EE, Spanjersberg SJ, de Roos A, Matheijssen NA, Zwinderman AH, van Dijkman PR, Reiber JH, Bruschke A V. Magnetic resonance imaging during dobutamine stress for detection and localization of coronary artery disease. Quantitative wall motion analysis using a modification of the centerline method. Circulation. 1994; 90:127-38.

55. Kuijpers D, Ho KYJ a M, van Dijkman PRM, Vliegenthart R, Oudkerk M. Dobutamine cardiovascular magnetic resonance for the detection of myocardial ischemia with the use of myocardial tagging. Circulation. 2003; 107:1592-7.

56. Korosoglou G, Gitsioudis G, Voss A, Lehrke S, Riedle N, Buss SJ, Zugck C, Giannitsis E, Osman NF, Katus H a. Strain-encoded cardiac magnetic resonance during high-dose dobutamine stress testing for the estimation of cardiac outcomes: comparison to clinical parameters and conventional wall motion readings. J Am Coll Cardiol. 2011; 58:1140-9.

57. Nagel E, Lehmkuhl HB, Bocksch W, Klein C, Vogel U, Frantz E, Ellmer A, Dreysse S, Fleck E. Noninvasive Diagnosis of Ischemia-Induced Wall Motion Abnormalities With the Use of High-Dose Dobutamine Stress MRI : Comparison With Dobutamine Stress Echocardiography. Circulation. 1999; 99:763-770.

58. Moonen M, Lancellotti P, Zacharakis D, Pierard L. The value of 2D strain imaging during stress testing. Echocardiography. 2009; 26:307-14. 
59. Moynihan PF, Parisi AF, Feldman CL. Quantitative detection of regional left ventricular contraction abnormalities by two-dimensional echocardiography. I. Analysis of methods. Circulation. 1981; 63:752-60.

60. Mor-Avi V, Vignon P, Koch R, Weinert L, Garcia MJ, Spencer KT, Lang RM. Segmental Analysis of Color Kinesis Images: New Method for Quantification of the Magnitude and Timing of Endocardial Motion During Left Ventricular Systole and Diastole. Circulation. 1997; 95:2082-97.

61. Wiske PS, Pearlman JD, Hogan RD, Franklin TD, Weyman AE. Echocardiographic definition of the left ventricular centroid. II. Determination of the optimal centroid during systole in normal and infarcted hearts. J Am Coll Cardiol. 1990; 16:993-9.

62. Urheim S, Edvardsen T, Torp H, Angelsen B, Smiseth OA. Myocardial strain by Doppler echocardiography. Validation of a new method to quantify regional myocardial function. Circulation. 2000; 102:1158-64.

63. Amundsen BH, Helle-Valle T, Edvardsen T, Torp H, Crosby J, Lyseggen E, Støylen A, Ihlen H, Lima JAC, Smiseth OA, Slørdahl SA. Noninvasive myocardial strain measurement by speckle tracking echocardiography: validation against sonomicrometry and tagged magnetic resonance imaging. J Am Coll Cardiol. 2006; 47:789-93.

64. Reant P, Labrousse L, Lafitte S, Bordachar P, Pillois X, Tariosse L, Bonoron-Adele S, Padois P, Deville C, Roudaut R, Dos Santos P. Experimental validation of circumferential, longitudinal, and radial 2-dimensional strain during dobutamine stress echocardiography in ischemic conditions. J Am Coll Cardiol. 2008; 51:149-57. 
65. Reant P, Labrousse L, Lafitte S, Tariosse L, Bonoron-Adele S, Padois P, Roudaut R, Dos Santos P, DeMaria A. Quantitative analysis of function and perfusion during dobutamine stress in the detection of coronary stenoses: two-dimensional strain and contrast echocardiography investigations. J Am Soc Echocardiogr. 2010; 23:95-103.

66. Voigt J-U, Exner B, Schmiedehausen K, Huchzermeyer C, Reulbach U, Nixdorff U, Platsch G, Kuwert T, Daniel WG, Flachskampf FA. Strain-rate imaging during dobutamine stress echocardiography provides objective evidence of inducible ischemia. Circulation. 2003; 107:2120-6.

67. Hanekom L, Cho G-Y, Leano R, Jeffriess L, Marwick TH. Comparison of twodimensional speckle and tissue Doppler strain measurement during dobutamine stress echocardiography: an angiographic correlation. Eur Heart J. 2007; 28:1765-72.

68. Mor-Avi V, Lang RM, Badano LP, Belohlavek M, Cardim NM, Derumeaux G, Galderisi M, Marwick T, Nagueh SF, Sengupta PP, Sicari R, Smiseth OA, Smulevitz B, Takeuchi M, Thomas JD, Vannan M, Voigt J-U, Zamorano JL. Current and Evolving Echocardiographic Techniques for the Quantitative Evaluation of Cardiac Mechanics: ASE/EAE Consensus Statement on Methodology and Indications Endorsed by the Japanese Society of Echocardiography. J Am Soc Echocardiogr. 2011; 24:277-313.

69. Corsi C, Lang RM, Veronesi F, Weinert L, Caiani EG, MacEneaney P, Lamberti C, Mor-Avi V. Volumetric quantification of global and regional left ventricular function from real-time three-dimensional echocardiographic images. Circulation. 2005; 112:1161-70. 
70. Walimbe V, Garcia M, Lalude O, Thomas J, Shekhar R. Quantitative real-time 3dimensional stress echocardiography: a preliminary investigation of feasibility and effectiveness. J Am Soc Echocardiog. 2007; 20:13-22.

71. Nesser J, Sugeng L, Corsi C, Weinert L, Niel J, Ebner C, Steringer-Mascherbauer R, Schmidt F, Schummers G, Lang RM, Mor-Avi V. Volumetric analysis of regional left ventricular function with real-time three-dimensional echocardiography: validation by magnetic resonance and clinical utility testing. Heart. 2007; 93:572-8.

72. Kuo J, Atkins B, Hutcheson K, von Ramm O. Left ventricular wall motion analysis using real-time three-dimensional ultrasound. Ultrasound Med Biol. 2005; 31:203-11.

73. Maffessanti F, Lang RM, Corsi C, Mor-Avi V, Caiani EG. Feasibility of left ventricular shape analysis from transthoracic real-time 3-D echocardiographic images. Ultrasound Med Biol. 2009; 35:1953-62.

74. Duan Q, Parker KM, Lorsakul A, Angelini ED, Hyodo E, Homma S, Holmes JW, Laine AF. Quantitative Validation of Optical Flow Based Myocardial Strain Measures Using Sonomicrometry. Proceedings IEEE International Symposium on Biomedical Imaging. 2009:454-457.

75. Seo Y, Ishizu T, Enomoto Y, Sugimori H, Yamamoto M, Machino T, Kawamura R, Aonuma K. Validation of 3-dimensional speckle tracking imaging to quantify regional myocardial deformation. Circ Cardiovasc Imaging. 2009; 2:451-9.

76. Seo Y, Ishizu T, Enomoto Y, Sugimori H, Aonuma K. Endocardial surface area tracking for assessment of regional LV wall deformation with 3D speckle tracking imaging. JACC Cardiovasc Imaging. 2011; 4:358-65. 
77. Herz SL, Hasegawa T, Makaryus AN, Parker KM, Homma S, Wang J, Holmes JW. Quantitative three-dimensional wall motion analysis predicts ischemic region size and location. Ann Biomed Eng. 2010; 38:1367-76.

78. Herz SL, Ingrassia CM, Homma S, Costa KD, Holmes JW. Parameterization of Left Ventricular Wall Motion for Detection of Regional Ischemia. Ann Biomed Eng. 2005; 33:912-19.

79. Ingrassia C, Usyk T, Kerckhoffs R, Mcculloch A, Costa K, Holmes J. Model-based development of four-dimensional wall motion measures. Comput Method Appl M. 2007; 196:3061-3069.

80. Herz SL. Quantitative Analysis of Three-Dimensional Left Ventricular Wall Motion for Diagnosis of Ischemia and Infarction. 2007:1-179.

81. Calnon DA, Glover DK, Beller GA, Vanzetto G, Smith WH, Watson DD, Ruiz M. Effects of dobutamine stress on myocardial blood flow, 99mTc sestamibi uptake, and systolic wall thickening in the presence of coronary artery stenoses: implications for dobutamine stress testing. Circulation. 1997; 96:2353-60.

82. Holmes JW. Candidate mechanical stimuli for hypertrophy during volume overload. J Appl Physiol. 2004; 97:1453-60.

83. Fomovsky GM, Clark SA, Parker KM, Ailawadi G, Holmes JW. Anisotropic reinforcement of acute anteroapical infarcts improves pump function. Circ Heart Fail. $2012 ; 5: 515-22$. 
84. Fomovsky GM. Scar Structure, Mechanics, and Ventricular Function in Healing Myocardial Infarction. 2010:1-195.

85. Lai M, Rubin D, Krempl E. Introduction to Continuum Mechanics.; 1999.

86. Ingul CB, Torp H, Aase SA, Berg S, Stoylen A, Slordahl SA. Automated analysis of strain rate and strain: feasibility and clinical implications. J Am Soc Echocardiog. 2005; 18:411-8.

87. Bjork Ingul C, Rozis E, Slordahl SA, Marwick TH. Incremental value of strain rate imaging to wall motion analysis for prediction of outcome in patients undergoing dobutamine stress echocardiography. Circulation. 2007; 115:1252-9.

88. Amundsen BH, Crosby J, Steen PA, Torp H, Slørdahl SA, Støylen A. Regional myocardial long-axis strain and strain rate measured by different tissue Doppler and speckle tracking echocardiography methods: a comparison with tagged magnetic resonance imaging. Eur J Echocardiogr. 2009; 10:229-37.

89. Heiberg E, Sjögren J, Ugander M, Carlsson M, Engblom H, Arheden H. Design and validation of Segment--freely available software for cardiovascular image analysis. BMC Med Imaging. 2010; 10:1.

90. Hashima AR, Young AA, McCulloch AD, Waldman LK. Nonhomogeneous analysis of epicardial strain distributions during acute myocardial ischemia in the dog. J Biomech. $1993 ; 26: 19-35$.

91. Bland JM, Altman DG. Statistical methods for assessing agreement between two methods of clinical measurement. Lancet. 1986; i:307-310. 
92. Mor-Avi V, Jenkins C, Kühl HP, Nesser H-J, Marwick T, Franke A, Ebner C, Freed BH, Steringer-Mascherbauer R, Pollard H, Weinert L, Niel J, Sugeng L, Lang RM. Realtime 3-dimensional echocardiographic quantification of left ventricular volumes:

multicenter study for validation with magnetic resonance imaging and investigation of sources of error. JACC Cardiovasc Imaging. 2008; 1:413-23.

93. Tanaka H, Oishi Y, Mizuguchi Y, Emi S, Ishimoto T, Nagase N, Tabata T, Yamada $\mathrm{H}$, Oki T. Three-dimensional evaluation of dobutamine-induced changes in regional myocardial deformation in ischemic myocardium using ultrasonic strain measurements: the role of circumferential myocardial shortening. J Am Soc Echocardiogr. 2007; 20:1294-99.

94. Korosoglou G, Lehrke S, Wochele A, Hoerig B, Lossnitzer D, Steen H, Giannitsis E, Osman NF, Katus HA. Strain-encoded CMR for the detection of inducible ischemia during intermediate stress. JACC Cardiovasc Imaging. 2010; 3:361-71.

95. Gallagher KP, Gerren RA, Choy M, Stirling MC, Dysko RC. Subendocardial segment length shortening at lateral margins of ischemic myocardium in dogs. Am J Physiol. 1987; 253:H826-37.

96. Van Leuven SL, Waldman LK, McCulloch AD, Covell JW. Gradients of epicardial strain across the perfusion boundary during acute myocardial ischemia. Am J Physiol. 1994; 267:H2348-62.

97. Gallagher K, Gerren R, Stirling M. The distribution of functional impairment across the lateral border of acutely ischemic myocardium. Circulation. 1986; 58:570-583. 
98. Leong-Poi H, Rim SE, Le DE, Fisher NG, Wei K, Kaul S. Perfusion versus function: the ischemic cascade in demand ischemia: implications of single-vessel versus multivessel stenosis. Circulation. 2002; 105:987-92.

99. Bogen DK, Rabinowitz SA, Needleman A, McMahon TA, Abelmann WH. An analysis of the mechanical disadvantage of myocardial infarction in the canine left ventricle. Circ Res. 1980; 47:728-741.

100. Dang ABC, Guccione JM, Zhang P, Wallace AW, Gorman RC, Gorman JH, Ratcliffe MB. Effect of ventricular size and patch stiffness in surgical anterior ventricular restoration: a finite element model study. Ann Thorac Surg. 2005; 79:185-93.

101. Wall ST, Walker JC, Healy KE, Ratcliffe MB, Guccione JM. Theoretical impact of the injection of material into the myocardium: a finite element model simulation. Circulation. 2006; 114:2627-35.

102. Kerckhoffs RCP, Omens JH, McCulloch AD, Mulligan LJ. Ventricular dilation and electrical dyssynchrony synergistically increase regional mechanical nonuniformity but not mechanical dyssynchrony: a computational model. Circ Heart Fail. 2010; 3:528-36.

103. Guccione JM, McCulloch AD, Waldman LK. Passive material properties of intact ventricular myocardium determined from a cylindrical model. J Biomech Eng. 1991; 113:42-55.

104. Costa KD, Hunter PJ, Rogers JM, Guccione JM, Waldman LK, McCulloch AD. A three-dimensional finite element method for large elastic deformations of ventricular myocardium: I--Cylindrical and spherical polar coordinates. J Biomech Eng. 1996; 118:452-63. 
105. Doll S, Schweizerhof K. On the development of volumetric strain energy functions. J Appl Mech. 2000; 67:17-21.

106. Lumens J, Delhaas T, Kirn B, Arts T. Three-wall segment (TriSeg) model describing mechanics and hemodynamics of ventricular interaction. Ann Biomed Eng. 2009; $37: 2234-55$.

107. Lin DH, Yin FC. A multiaxial constitutive law for mammalian left ventricular myocardium in steady-state barium contracture or tetanus. J Biomech Eng. 1998; 120:504-17.

108. Arts T, Delhaas T, Bovendeerd P, Verbeek X, Prinzen FW. Adaptation to mechanical load determines shape and properties of heart and circulation: the CircAdapt model. Am J Physiol Heart Circ Physiol. 2005; 288:H1943.

109. Yip G, Khandheria B, Belohlavek M, Pislaru C, Seward J, Bailey K, Tajik a J, Pellikka P, Abraham T. Strain echocardiography tracks dobutamine-induced decrease in regional myocardial perfusion in nonocclusive coronary stenosis. J Am Coll Cardiol. $2004 ; 44: 1664-71$.

110. Guccione J, Costa K, McCulloch A. Finite element stress analysis of left ventricular mechanics in the beating dog heart. J Biomech. 1995; 28:1167-1177.

111. Costa KD, Hunter PJ, Wayne JS, Waldman LK, Guccione JM, McCulloch AD. A three-dimensional finite element method for large elastic deformations of ventricular myocardium: II--Prolate spheroidal coordinates. J Biomech Eng. 1996; 118:464-72. 
112. Costa KD, Takayama Y, McCulloch AD, Covell JW. Laminar fiber architecture and three-dimensional systolic mechanics in canine ventricular myocardium. Am J Physiol. 1999; 276:H595-607.

113. Rodriguez F, Langer F, Harrington KB, Cheng A, Daughters GT, Criscione JC, Ingels NB, Miller DC. Alterations in transmural strains adjacent to ischemic myocardium during acute midcircumflex occlusion. J Thorac Cardiov Sur. 2005; 129:791-803.

114. Falsetti H, Mates R, Greene D, Bunnell IL. Vmax Index of Contractile State in Man. Circulation. 1971; 43:467-479.

115. Kaseda S, Tomoike H, Ogata I, Nakamura M. End-systolic pressure-volume, pressure-length, and stress-strain relations in canine hearts. Am J Physiol Heart Circ Physiol. 1985; 249:H648.

116. Marwick TH. Measurement of strain and strain rate by echocardiography: ready for prime time? J Am Coll Cardiol. 2006; 47:1313-27.

117. Bansal M, Cho G-Y, Chan J, Leano R, Haluska BA, Marwick TH. Feasibility and accuracy of different techniques of two-dimensional speckle based strain and validation with harmonic phase magnetic resonance imaging. J Am Soc Echocardiogr. 2008; $21: 1318-25$.

118. Uematsu M, Miyatake K, Tanaka N, Matsuda H, Sano A, Yamazaki N, Hirama M, Yamagishi M. Myocardial velocity gradient as a new indicator of regional left ventricular contraction: detection by a two-dimensional tissue Doppler imaging technique. J Am Coll Cardiol. 1995; 26:217-23. 
119. Gallagher K, Matsuzaki M, Koziol J, WS K, Ross J. Regional myocardial perfusion and wall thickening during ischemia in conscious dogs. Am J Physiol. 1984; 247:H72738.

120. Leong-Poi H, Coggins MP, Sklenar J, Jayaweera AR, Wang X-Q, Kaul S. Role of collateral blood flow in the apparent disparity between the extent of abnormal wall thickening and perfusion defect size during acute myocardial infarction and demand ischemia. J Am Coll Cardiol. 2005; 45:565-72.

121. Chang SA, Lee SC, Kim EY, Hahm SH, Jang SY, Park SJ, Choi JO, Park SW, Choe YH, Oh JK. Feasibility of Single-Beat Full-Volume Capture Real-Time ThreeDimensional Echocardiography and Auto-Contouring Algorithm for Quantification of Left Ventricular Volume: Validation with Cardiac Magnetic Resonance Imaging. J Am Soc Echocardiog. 2011; C.

122. Leung KYE, Danilouchkine MG, van Stralen M, de Jong N, van der Steen AFW, Bosch JG. Left ventricular border tracking using cardiac motion models and optical flow. Ultrasound Med Biol. 2011; 37:605-16.

123. Yodwut C, Weinert L, Klas B, Lang RM, Mor-Avi V. Effects of frame rate on threedimensional speckle-tracking-based measurements of myocardial deformation. J Am Soc Echocardiogr. 2012; 25:978-85.

124. Obuchowski NA, Lieber ML, Wians FH. ROC curves in clinical chemistry: uses, misuses, and possible solutions. Clin Chem. 2004; 50:1118-25.

125. Mahrholdt H, Zhydkov A, Hager S, Meinhardt G, Vogelsberg H, Wagner A, Sechtem U. Left ventricular wall motion abnormalities as well as reduced wall thickness 
can cause false positive results of routine SPECT perfusion imaging for detection of myocardial infarction. Eur Heart J. 2005; 26:2127-35.

126. Gupta KB, Ratcliffe MB, Fallert M a, Edmunds LH, Bogen DK. Changes in passive mechanical stiffness of myocardial tissue with aneurysm formation. Circulation. 1994; 89:2315-26.

127. Holmes JW, Borg TK, Covell JW. Structure and mechanics of healing myocardial infarcts. Annu Rev Biomed Eng. 2005; 7:223-53.

128. Fomovsky GM, Holmes JW. Evolution of scar structure, mechanics, and ventricular function after myocardial infarction in the rat. Am J Physiol Heart Circ Physiol. 2010; 298:H221-8.

129. Fomovsky GM, Macadangdang JR, Ailawadi G, Holmes JW. Model-Based Design of Mechanical Therapies for Myocardial Infarction. J Cardiovasc Trans Res. 2011; 4:8291.

130. Patel RAG, Glover DK, Broisat A, Kabul HK, Ruiz M, Goodman NC, Kramer CM, Meerdink DJ, Linden J, Beller GA. Reduction in myocardial infarct size at 48 hours after brief intravenous infusion of ATL-146e, a highly selective adenosine A2A receptor agonist. Am J Physiol Heart Circ Physiol. 2009; 297:H637-42.

131. VAN RUGGE FP, HOLMAN ER, VAN DER WALL EE, DE ROOS A, VAN DER LAARSE A, BRUSCHKE AVG. Quantitation of global and regional left ventricular function by cine magnetic resonance imaging during dobutamine stress in normal human subjects. Eur Heart J. 1993; 14:456-463. 
132. Pennell DJ, Firmin DN, Burger P, Yang GZ, Manzara CC, Ell PJ, Swanton RH, Walker JM, Underwood SR, Longmore DB. Assessment of magnetic resonance velocity mapping of global ventricular function during dobutamine infusion in coronary artery disease. Br Heart J. 1995; 74:163-70.

133. Pellikka PA, Roger VL, McCully RB, Mahoney DW, Bailey KR, Seward JB, Tajik AJ. Normal stroke volume and cardiac output response during dobutamine stress echocardiography in subjects without left ventricular wall motion abnormalities. Am J Cardiol. 1995; 76:881-6.

134. Tanimoto M, Pai RG, Jintapakorn W. Normal changes in left ventricular filling and hemodynamics during dobutamine stress echocardiography. J Am Soc Echocardiog. 1995; 8:488-93.

135. Carstensen S, Ali SM, Stensgaard-Hansen F V., Toft J, Haunso S, Kelbæk H, Saunamaki K. Dobutamine-Atropine Stress Echocardiography in Asymptomatic Healthy Individuals : The Relativity of Stress-Induced Hyperkinesia. Circulation. 1995; 92:34533463.

136. Morise AP, Haddad WJ, Beckner D. Development and validation of a clinical score to estimate the probability of coronary artery disease in men and women presenting with suspected coronary disease. Am J Med. 1997; 102:350-356.

137. Cerqueira MD, Weissman NJ, Dilsizian V, Jacobs AK, Kaul S, Laskey WK, Pennell DJ, Rumberger JA, Ryan T, Verani MS. Standardized myocardial segmentation and nomenclature for tomographic imaging of the heart. A statement for healthcare 
professionals from the Cardiac Imaging Committee of the Council on Clinical Cardiology of the American Heart Association. Circulation. 2002; 105:539-42.

138. Shahgaldi K, Gudmundsson P, Manouras A, Brodin L-A, Winter R. Visually estimated ejection fraction by two dimensional and triplane echocardiography is closely correlated with quantitative ejection fraction by real-time three dimensional echocardiography. Cardiovasc Ultrasound. 2009; 7:41.

139. YAMASHITA K, TAKEUCHI M, TSUDA Y, SONODA S, MIURA Y, ISHINO Y, NAKASHIMA Y. Does the Lack of Hyperkinesis During Dobutamine Stress Echocardiography Predict the Functional Significance of Coronary Arterial Stenosis? Echocardiography. 2000; 17:229-239.

140. McNeill AJ, Fioretti PM, El-Said SM, Salustri A, Forster T, Roelandt JR. Enhanced sensitivity for detection of coronary artery disease by addition of atropine to dobutamine stress echocardiography. Am J Cardiol. 1992; 70:41-6.

141. Kuijer JPA, Marcus JT, Götte MJW, van Rossum AC, Heethaar RM. Threedimensional myocardial strains at end-systole and during diastole in the left ventricle of normal humans. J Cardiov Magn Reson. 2002; 4:341-51.

142. Bristow MR, Saxon LA, Boehmer J, Krueger S, Kass DA, De Marco T, Carson P, DiCarlo L, DeMets D, White BG, DeVries DW, Feldman AM. Cardiac-resynchronization therapy with or without an implantable defibrillator in advanced chronic heart failure. $\mathrm{N}$ Engl J Med. 2004; 350:2140-50. 
143. Cleland JGF, Daubert J-C, Erdmann E, Freemantle N, Gras D, Kappenberger L, Tavazzi L. The effect of cardiac resynchronization on morbidity and mortality in heart failure. N Engl J Med. 2005; 352:1539-49.

144. Chung ES, Leon AR, Tavazzi L, Sun J-P, Nihoyannopoulos P, Merlino J, Abraham WT, Ghio S, Leclercq C, Bax JJ, Yu C-M, Gorcsan J, St John Sutton M, De Sutter J, Murillo J. Results of the Predictors of Response to CRT (PROSPECT) trial. Circulation. 2008; 117:2608-16.

145. Helm RH, Byrne M, Helm PA, Daya SK, Osman NF, Tunin R, Halperin HR, Berger RD, Kass DA, Lardo AC. Three-dimensional mapping of optimal left ventricular pacing site for cardiac resynchronization. Circulation. 2007; 115:953-61.

146. Singh JP, Klein HU, Huang DT, Reek S, Kuniss M, Quesada A, Barsheshet A, Cannom D, Goldenberg I, McNitt S, Daubert JP, Zareba W, Moss AJ. Left ventricular lead position and clinical outcome in the multicenter automatic defibrillator implantation trial-cardiac resynchronization therapy (MADIT-CRT) trial. Circulation. 2011; 123:1159_ 66.

147. Spragg DD, Dong J, Fetics BJ, Helm R, Marine JE, Cheng A, Henrikson CA, Kass DA, Berger RD. Optimal left ventricular endocardial pacing sites for cardiac resynchronization therapy in patients with ischemic cardiomyopathy. J Am Coll Cardiol. 2010; 56:774-81.

148. Derval N, Steendijk P, Gula LJ, Deplagne A, Laborderie J, Sacher F, Knecht S, Wright M, Nault I, Ploux S, Ritter P, Bordachar P, Lafitte S, Réant P, Klein GJ, Narayan SM, Garrigue S, Hocini M, Haissaguerre M, Clementy J, Jaïs P. Optimizing 
hemodynamics in heart failure patients by systematic screening of left ventricular pacing sites: the lateral left ventricular wall and the coronary sinus are rarely the best sites. $\mathrm{J}$ Am Coll Cardiol. 2010; 55:566-75.

149. Ypenburg C, Roes SD, Bleeker GB, Kaandorp TA, de Roos A, Schalij MJ, van der Wall EE, Bax JJ. Effect of total scar burden on contrast-enhanced magnetic resonance imaging on response to cardiac resynchronization therapy. Am J Cardiol. 2007; 99:65760.

150. White JA, Yee R, Yuan X, Krahn A, Skanes A, Parker M, Klein G, Drangova M. Delayed enhancement magnetic resonance imaging predicts response to cardiac resynchronization therapy in patients with intraventricular dyssynchrony. J Am Coll Cardiol. 2006; 48:1953-60.

151. Bleeker GB, Schalij MJ, Van Der Wall EE, Bax JJ. Postero-lateral scar tissue resulting in non-response to cardiac resynchronization therapy. J Cardiovasc Electrophysiol. 2006; 17:899-901.

152. Chalil S, Stegemann B, Muhyaldeen S, Khadjooi K, Foley P, Smith R, Leyva F. Effect of posterolateral left ventricular scar on mortality and morbidity following cardiac resynchronization therapy. Pacing Clin Electrophysiol. 2007; 30:1201-9.

153. Rhode KS, Sermesant M, Brogan D, Hegde S, Hipwell J, Lambiase P, Rosenthal E, Bucknall C, Qureshi SA, Gill JS, Razavi R, Hill DLG. A system for real-time XMR guided cardiovascular intervention. IEEE Trans Med Imaging. 2005; 24:1428-40.

154. Duckett SG, Ginks MR, Knowles BR, Ma Y, Shetty A, Bostock J, Cooklin M, Gill JS, Carr-White GS, Razavi R, Schaeffter T, Rhode KS, Rinaldi CA. Advanced image fusion 
to overlay coronary sinus anatomy with real-time fluoroscopy to facilitate left ventricular lead implantation in CRT. Pacing Clin Electrophysiol. 2011; 34:226-34.

155. Ypenburg C, van Bommel RJ, Delgado V, Mollema SA, Bleeker GB, Boersma E, Schalij MJ, Bax JJ. Optimal left ventricular lead position predicts reverse remodeling and survival after cardiac resynchronization therapy. J Am Coll Cardiol. 2008; 52:1402-9.

156. MacKay SA, Potel MJ, Rubin JM. Graphics methods for tracking three-dimensional heart wall motion. Comput Biomed Res. 1982; 15:455-73.

157. Rhode KS, Hill DLG, Edwards PJ, Hipwell J, Rueckert D, Sanchez-Ortiz G, Hegde S, Rahunathan V, Razavi R. Registration and tracking to integrate X-ray and MR images in an XMR facility. IEEE Trans Med Imaging. 2003; 22:1369-78.

158. Ector J, De Buck S, Huybrechts W, Nuyens D, Dymarkowski S, Bogaert J, Maes F, Heidbüchel H. Biplane three-dimensional augmented fluoroscopy as single navigation tool for ablation of atrial fibrillation: accuracy and clinical value. Heart Rhythm. 2008; 5:957-64.

159. Saito K, Okura H, Watanabe N, Hayashida A, Obase K, Imai K, Maehama T, Kawamoto T, Neishi Y, Yoshida K. Comprehensive evaluation of left ventricular strain using speckle tracking echocardiography in normal adults: comparison of threedimensional and two-dimensional approaches. J Am Soc Echocardiog. 2009; 22:102530 .

160. Mele D, Agricola E, Monte AD, Galderisi M, D'Andrea A, Rigo F, Citro R, Chiodi E, Marchese G, Valentina P Della, Calabrese A, Ferrari R. Pacing transmural scar tissue 
reduces left ventricle reverse remodeling after cardiac resynchronization therapy. Int $J$ Cardiol. 2012. 